\title{
Interaction of attosecond and femtosecond pulses with atoms and molecules
}

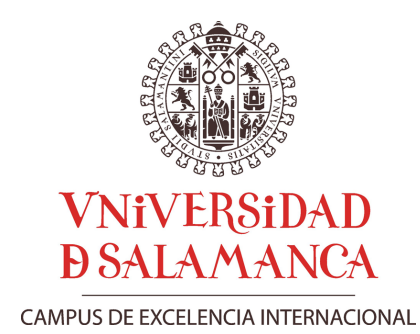

Alexis Agustín Chacón Salazar

FACULTAD DE CIENCIAS

Departamento de Física Aplicada. Área de Óptica.

Universidad de Salamanca

A thesis submitted for the degree of

Doctor in Physics at Universidad de Salamanca

Supervisor: Dr. Camilo Ruiz Méndez

Co-supervisor: Prof. Dr. Luis Plaja Rustein

Salamanca, December 2014 
CAMILO RUIZ MÉNDEZ, Investigador Asociado del Departamento de Física de Partículas en la Universidad de Santiago de Compostela y

LUIS PLAJA RUSTEIN, Profesor Titular del Área de Óptica del Departamento de Física Aplicada de la Universidad de Salamanca,

CERTIFICAN:

Que la presente Memoria, "Interaction of attosecond and femtosecond pulses with atoms and molecules" ha sido realizada bajo su dirección y turoría en el Área de Óptica del Departamento de Física Aplicada de la Universidad de Salamanca por Don Alexis Agustín Chacón Salazar y constituye su Tesis Doctoral para optar al grado de Doctor en Física por la Universidad de Salamanca.

Camilo Ruiz Méndez

Director
Luis Plaja Rustein

Tutor

Salamanca, 12 de diciembre de 2014. 
"The ultimate value of life depends upon awareness and the power of contemplation rather than upon mere survival."

Aristotle 
To the memory of my beloved brother: Ricardo A. Chacón S.

To my parents: Agustín and Vielka To my siblings: María and Agustín Junior To my wife: Alma Dreyer 


\section{Acknowledgements}

First, I would like to thank my beloved parents, Agustín and Vielka, simply because I owe them everything I have achieved. Their example has taught me to face life with courage and love.

Second, I would like to thank Prof. Dr. Luis Plaja Rustein for supporting my application for the fellowship from the Secretaría Nacional de Ciencia, Tecnología e Innovación of Panama. This gave me the opportunity to get the financial support for my PhD studies in El Área de Óptica de la Universidad de Salamanca. I also thank you for all the wonderful discussions about physics.

I am very grateful to Dr. Camilo Ruiz for the supervision of my thesis. I really learned a lot from you: physical concepts, powerful computational tools, communication strategies and great research ideas. In addition, thank you very much for your patience and support during the good and difficult times of this phase in my professional life.

I also want to thank Prof. Dr. Manfred Lein for the great support and hospitality during my visits to his research group. I have enjoyed our discussions about physics and learned a lot from them. Furthermore, I would like to extend my gratitude to Jost H., Ingo P., Jing Z., María T. and Chirag J. from Lein's group, for the pleasant moments that we shared.

I have to mention a modest person, with whom I have been working very closely throughout this thesis. He helped me to understand many computational tools in detail and with great patience. Rafael Morán, thank you so much for being a great friend and co-worker.

Five years ago, I had the luck to become part of a great group: El Fotón Charro. I thank all of you: Cruz M., Ana G., Isabel A., Enrique C., Pablo M., Javier R., Iñigo S., Julio S.-R., Oscar V., Carolina R. and Luis P., for the uncountable meetings where we shared great moments. I have learned a lot about the Spanish culture.

I will also remember all the nice moments that I shared with my friends and PhD "brothers": José Antonio P., Benjamín A., Carlos H., Rocío B., 
Julio R., Warein O., Isabel G., José Luis H., Alejandro de la C., Enrique G., Camilo G., Fran V. and René R.

I also want to thank El Centro de Láseres Pulsados for opening its doors and providing the required computational tools for my research: Luis R., Javier Sastre, Yaiza C., Mauricio R., Alvaro P., Jon A., Juan H., Diego A., Javier S., Cristina M. and all the others I had the pleasure to work with. Especially, I would like to mention Luca S. and Andreas D., thanks for all the great moments that you allowed me to share with you. I have enjoyed your viewpoints about life and learned from them.

I want to thank Ricardo T., for the great philosophical discussions about physics and most importantly, for your advices at difficult times during this period.

I also want to mention a person with whom I have collaborated during the last year. I have enjoyed our discussions and learned a lot from him. Marcelo C., thank you so much for being a great co-worker.

I would like to thank my whole family from Panama. In particular, I am very grateful to my uncles Luis C., Victor S. and cousin Migdalia R., to believe in me. Thanks a lot for all your support in helping me to obtain the scholarship for my PhD studies.

I also thank all my friends: Eva, Jesse, Álvaro, Nacho, Julia, Alberto, Gema, Daniel, Anne, Silvia, Raúl, Xiomy, Luis,... for all those great memories. I want to thank my yoga teacher, Maura D., for all her advice and her great life energy.

I know that you are not here with us, but I want to mention your memory, my beloved brother Ricardo Ch. Thanks so much for the great motivation words that you gave me during this thesis time before your death. Your courage to face life, your love for the salsa music, for our friends and for our parents will be with me forever.

Finally, I deeply thank my beloved wife, Alma, for her support during this long time, her patience, her company during the happy and hard moments of this thesis period. You are the inspiration of my poems.

I thank LIFE for my health and for having enough energy to meet this challenge and to fulfill one of my dreams: my $\mathrm{PhD}$. 


\begin{abstract}
Nowadays attosecond science tools have opened new avenues to access the ultrafast electronic motion in matter with unprecedented time and spatial resolutions.

This thesis is dedicated to the study of both the structural and dynamical information carried by the electrons in atoms and small molecules. Our first aim consists in applying the schemes widely used in optics to reconstruct the amplitude and phase of the ultrafast laser pulses. Analogously, we extend these schemes to the matter-wave characterization. By using the attosecond streak camera concepts, we theoretically demonstrate that the application of two attosecond pulses, in the presence of a moderated infrared laser field, leads to the emission of two coherent electron wavepackets (EWP). The resulting interferogram contains full information about the phase difference between the two ionization events. Conceptually, this ionization scheme is equivalent to the optical technique named Spectral Phase Interferometry for Direct Electric-field Reconstruction (SPIDER). In a similar way to the one used in optics, we apply the SPIDER algorithm but in this case to extract information of an atomic system. This information is encoded in the complex bound-free dipole transition matrix element, amplitude and phase, and can be obtained from the final interferogram.

Furthermore, the time delay in photoemission or Wigner time delay is a fundamental quantity related to the time-dynamics of an EWP when it is ionized by a single attosecond pulse. The now available attosecond streaking technique has demonstrated to be the adequate tool to extract the time delay in the photoionization process. However, due to the problems related to the Coulomb-laser coupling (CLC), the information of the intrinsic Wigner time delay is intertwined in the measurement process. In this thesis we will evaluate an alternative method to remove the CLC from the measurement process of the Wigner time delay and address its reliability. To this end we use a small asymmetric molecule. By measuring two streaking traces, to either side of the molecular orientation axis, the resulting left-right time delay is obtained. We demonstrate that this simple asymmetric time delay removes completely the so-problematic CLC. At the same time, our numerical calculations suggest that the exact asymmetric Wigner time delay is comparable to the stereo streaking delay.
\end{abstract}




\section{Resumen}

Hoy en día las herramientas de la ciencia de attosegundos han abierto nuevas vías para acceder al movimiento electrónico ultra-rápido en la materia con resoluciones temporales y espaciales por primera vez observadas.

La presente tesis está dedicada al estudio de la información estructural y dinámica asociada a los electrones en átomos y moléculas. Nuestro primer propósito consiste en aplicar algunos esquemas usados en Óptica para reconstruir la amplitud y la fase de pulsos láseres ultra-rápidos. Análogamente y desde un punto de vista teórico, nosotros extendemos éstos esquemas a la caracterización de ondas de materia. Mediante el uso de los conceptos de la attosecond streak camera, demostramos que la aplicación de dos pulsos de attosegundos en presencia de un pulso láser en el régimen del infrarrojo y con intensidad moderada, conduce a la emisión de dos paquetes de ondas de electrónes (EWP). El interferograma resultante contiene información completa acerca de la diferencia de fase entre los dos eventos de ionización. Conceptualmente, dicho esquema de ionización es equivalente a la técnica de caracterización en Óptica denominada Spectral Phase Interferometry for Direct Electric-field Reconstruction (SPIDER). De manera semejante a la seguida en Ópitca, aplicamos el algoritmo SPIDER, pero en nuestro caso con el propósito de recuperar información estructural de sistemas atómicos. Dicha información está codificada en el elemento de transición dipolar complejo entre los estados ligados y los del continuo. Esta información puede ser obtenida a partir del interferograma de paquetes de ondas electrónicos.

Por otro lado, el retado en la foto-emisión o tiempo de Wigner es una cantidad fundamental relacionada con la dinámica de un EWP cuando éste es ionizado mediante un pulso de attosegundos. La técnica attosecond streaking ha demostrado ser la herramienta adecuada para extraer el retardo en el proceso de foto-emisión. Sin embargo, debido al acoplamiento entre el potencial de Coulomb y el láser (CLC), la información del tiempo de Wigner se observa mezclada en el proceso de medición. Como segundo propósition, en esta tesis se sugiere un método alternativo para remover el CLC en el proceso de medición del tiempo de Wigner. Con tal fin, usamos una molécula asimétrica "pequeña". Mediante la medición de dos trazas streaking, una a la izquierda y otra a la derecha con respecto al eje de orientación molecular, obtenemos el retardo temporal asimétrico izquiera-derecha. Con ello, demostramos que esta simple asimetría en la medición de los retardos remueve completamente el llamado CLC. Simultáneamente, nuestros cálculos numéricos sugieren que la asimetría en el tiempo de Wigner es comparable al retardo relativo izquierda-derecha obtenido con el método streaking. 


\section{List of Publications}

The report of this thesis is based on the following papers:

I Asymmetry of Wigner's time delay in a small molecule

A. Chacón, M. Lein and C. Ruiz. Phys. Rev. A 89, 053427 (2014).

(Paper published as Editors' suggestion)

II Retrieval of the amplitude and phase of the dipole matrix element by attosecond electron-wave-packet interferometry A. Chacón, M. Lein and C. Ruiz. Phys. Rev. A 87, 023408 (2013).

III Effects of electron dynamics in the measurement of the delay in photoemission using streaking technique

A. Chacón and C. Ruiz. Paper in preparation.

IV Momentum-spatial phase retrieval of electron wavepackets using elliptical fields

A. Chacón and C. Ruiz. Paper in preparation.

Other work carried out in the predoctoral period and not mentioned in this report:

I High harmonic generation enhanced by coherent population return

A. Chacón, M. F. Ciappina and A. Peralta Conde. Submitted paper. 


\section{Acronyms}

1D, 2D and 3D One Dimensional, Two Dimensional and Three Dimensional

$\mathrm{AM}$

APT

ATI

CLC

$\mathrm{CPA}$

$\mathrm{CEP}$

EWP

FROG-CRAB

FWHM

FT

FFT

FFTW

IR

HHG

HOMO

HT

LIC

MKL

PW

QSPIDER

RABBIT

SAE

SAP

SFA

SPIDER
Asymmetric Molecule

Attosecond Pulse Train

Above Threshold Ionization

Coulomb-Laser-Coupling

Chirped Pulse Amplification

Carrier Envelope Phase

Electron Wave Packet

Frequency Resolved Optical Gating

for Complete Reconstruction of Attosecond Bursts

Full Width at Half Maximum

Fourier Transform

Fast Fourier Transform

Fast Fourier Transform in the West

Infra Red

High-order Harmonic Generation

Highest Occupied Molecular Orbital

Hankel Transform

Laser Induced Chirp

Math Kernel Library

Plane Wave

Quantum Spectral Phase Interferometry for

Direct Electron-wavepacket Reconstruction

Reconstruction of Attosecond Beating By

Interference of Two-photon transitions

Single Active Electron

Single Attosecond Pulse

Strong Field Approximation

Spectral Phase Interferometry for Direct

Electric-field Reconstruction

SW

Scattering Wave 
SWTD Stereo Wigner Time Delay

SSTD Stereo Streaking Time Delay

TDSE Time Dependent Schrödinger Equation

TISE Time Independent Schrödinger Equation

TOF Time Of Flight

WKB Wentzel-Kramers-Brillouin approximation

XUV eXtreme Ultra Violet 


\section{Contents}

List of Publications $\quad$ ix

Acronyms $\quad x$

Contents xiii

List of Figures $\quad$ xvii

1 Introduction $\quad 1$

1.1 Brief history of ultra-short laser pulses . . . . . . . . . . . . 3

1.2 High-order harmonic generation . . . . . . . . . . . . . . . . 6

1.3 Attosecond pulse generation . . . . . . . . . . . . . . . 10

1.4 State-of-the-art in attosecond physics . . . . . . . . . . . . . 13

1.5 Aim of the thesis . . . . . . . . . . . . . . . 16

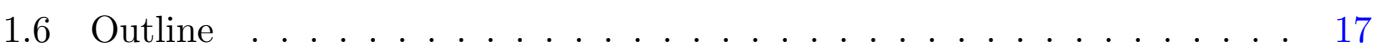

2 Theoretical and numerical methods $\quad 19$

2.1 Strong field interaction . . . . . . . . . . . . . . . . . . . 19

2.1.1 Schrödinger formalism . . . . . . . . . . . . . . . . . 21

2.1.2 Time evolution operator . . . . . . . . . . . . . . 23

2.1.3 Time independent Hamiltonians . . . . . . . . . . . . . . 25

2.1 .4 Time dependent Hamiltonians _. . . . . . . . . . . . 25

2.1.5 Observables in Quantum Mechanics . . . . . . . . . . . . 26

2.1.6 Strong field approximation . . . . . . . . . . . . 26

2.1.6.1 Volkov states . . . . . . . . . . . . . . 27

2.1.6.2 Complex transition matrix element . . . . . . . 28

2.2 Numerical methods . . . . . . . . . . . . . . . . . . . . . . . 31

2.2.1 Schrödinger equation in cartesian coordinates . . . . . . . . . 31 
2.2.1.1 Split-operator spectral method . . . . . . . . . . 32

2.2.1.2 Position and momentum space: the grids . . . . . . . . 34

2.2.1.3 Hamiltonian of the Systems . . . . . . . . . . . . . . . 35

2.2.1.4 Discrete and continuum spectrum . . . . . . . . 36

2.2.1.5 Electric-field of the laser pulses . . . . . . . . . . . . 42

2.2.1.6 Time dependent evolution . . . . . . . . . . . . . . 43

2.2.1.7 Absorbing boundaries . . . . . . . . . . . . . . . . 44

2.2.1.8 Observables . . . . . . . . . . . . . . . . 44

2.2.2 Schrödinger equation in cylindrical coordinates . . . . . . . . . 47

2.2.2.1 Crank-Nicolson methods in cylindrical coordinates . . . 48

2.2.2.2 Numerical aspects in cylindrical coordinates . . . . . . 51

2.2.3 Numerical integration: ionization transition amplitude . . . . . . 56

2.2.3.1 Runge-Kutta method . . . . . . . . . . . 56

2.2.3.2 Momentum grid definition in SFA . . . . . . . . . 57

3 Software design $\quad 59$

3.1 Introduction . . . . . . . . . . . . . . . . . . . . 59

3.1.1 Library structures according to physics concepts . . . . . . . . 60

3.1 .2 Basic numerical tools splitting . . . . . . . . . . . . . 62

3.1 .3 Cycle of Ideas . . . . . . . . . . . . . . . . . . . . . . 64

3.2 PULSESMAKER . . . . . . . . . . . . . . . . . . 65

3.2 .1 Time grid array . . . . . . . . . . . . . . 67

3.2 .2 Time object class . . . . . . . . . . . . . . . . . . . 67

$3.2 .3 \quad$ Laser class . . . . . . . . . . . . . . . . . . . . . . . 68

3.3 QFISHBOWL . . . . . . . . . . . . . . . . . . 69

3.3 .1 Computational grid class . . . . . . . . . . . . . 71

3.3.2 Wavefunction class . . . . . . . . . . . . . . . 73

3.3.3 Hamiltonian or potential class . . . . . . . . . . . . . . . . . . 74

3.3.4 Evolution operators: cartesian class . . . . . . . . . . . 75

3.4 BALAS . . . . . . . . . . . . . . . . . . . 78

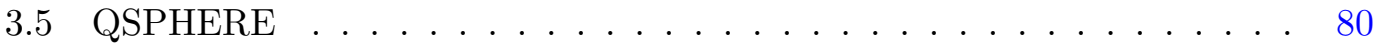

4 Amplitude and phase retrieval techniques: theoretical viewpoint 83

4.1 Introduction . . . . . . . . . . . . . . . . . . 83

4.2 Quantum spectral phase interferometry for direct EWP reconstruction . 85 4.2.1 Photoionization by a single attosecond pulse . . . . . . . . . 88 
4.2.2 Photoionization by two delayed attosecond pulses . . . . . . . . . 92

4.2.3 Photoionization in presence of an infrared laser . . . . . . . . . . 95

4.2.4 Attosecond electron wave packet interferometry . . . . . . . . . . 99

4.2.5 Coulomb laser coupling phase . . . . . . . . . . . . . . . . . 102

4.2 .6 QSPIDER implementation . . . . . . . . . . . . . . . . . 104

4.2.7 Results within Strong Field Approximation . . . . . . . . . . 107

4.2.8 Results within the Time Dependent Schrödinger Equation . . . . 114

4.3 Lateral momentum-shearing EWP interferometry technique . . . . . . . 121

4.3.1 Two dimensional electron wave packet interferometry . . . . . . 125

4.3.2 QSPIDER and 2D lateral momentum-shearing implementation . 128

4.3.3 Results within SFA and TDSE models . . . . . . . . . . . . . 132

4.3.3.1 Lateral momentum-shearing results within SFA . . . 132

4.3.3.2 Time dependent Schrödinger equation model . . . . . . 137

4.4 Conclusions . . . . . . . . . . . . . . . . . . . . . . . . . 142

5 Delay in photoemission $\quad 145$

5.1 Introduction . . . . . . . . . . . . . . . . . . . . . . . 145

5.2 Wigner time in atoms . . . . . . . . . . . . . . . . 147

5.2.1 Wigner time by quantum and classical pictures . . . . . . . . . 149

5.2.2 Measurement of Wigner time by streaking techniques . . . . . 153

5.3 Wigner time delay in a small asymmetric molecule . . . . . . . . . . . . 161

5.3.1 Description of the asymmetric system . . . . . . . . . . 162

5.3.2 Dipole structure and stereo Wigner time . . . . . . . . . . . 163

5.3.3 Photoionization form an asymmetric molecule . . . . . . . . . 165

5.3.4 Stereo streaking technique in an asymmetric molecule . . . . . . 171

5.4 Asymmetric molecule in three dimensions . . . . . . . . . . . . . . . . 175

5.4 .1 Definition of the system . . . . . . . . . . . . . . 177

5.4 .2 Stereo TOF: 3D results . . . . . . . . . . . . . . . . 179

5.4.3 Stereo streaking technique under the three dimensional TDSE . . 182

5.4.3.1 Molecular bound-bound electron dynamics . . . . . . . 192

5.5 Conclusions . . . . . . . . . . . . . . . . . . . . . . 195

$\begin{array}{lr}\text { Conclusions and outlook } & 197\end{array}$

$\begin{array}{lr}\text { Appendix A } & 201\end{array}$

A.1 Semiclassical aspects of the delay in photoemission . . . . . . . . . . . 201

A.2 Extrapolation method . . . . . . . . . . . . . . . . 202 
A.3 Stationary phase method . . . . . . . . . . . . . . . . 203

A.4 Photoionization via a single attosecond pulse . . . . . . . . . . . 205

$\begin{array}{ll}\text { Bibliography } & 211\end{array}$ 


\section{List of Figures}

1.1 Timescale of different physical phenomena. . . . . . . . . . . . . 2

1.2 Evolution of ultrafast laser pulses. . . . . . . . . . . . . . 4

1.3 Classical scheme of $\mathrm{HHG}$ process. . . . . . . . . . . . . . . 8

1.4 Strong field ionization and HHG spectrum. . . . . . . . . . . . . . 9

1.5 Attosecond pulses train. . . . . . . . . . . . . . . . . 11

1.6 Single attosecond pulse. . . . . . . . . . . . . . . . . . . 12

2.1 Scattering wave in 1D. . . . . . . . . . . . . . 41

3.1 Designing code within physics concepts. . . . . . . . . . . . . . . . 61

3.2 Software architecture: flexible design. . . . . . . . . . . . . . 63

3.3 Cycle of ideas. . . . . . . . . . . . . . . . . 64

3.4 PULSESMAKER general design. . . . . . . . . . . . . . . . 66

3.5 Time grid class. . . . . . . . . . . . . . . . . . 67

3.6 Laser class. . . . . . . . . . . . . . . . . . . . . . . . . . . . . . 68

3.7 QFISHBOWL library scheme. . . . . . . . . . . . . . . . . . . . . . . . . . . 70

3.8 Grid class diagram. . . . . . . . . . . . . . . . . . . . . 72

3.9 Wavefunction class. . . . . . . . . . . . . . . . . . 73

3.10 "Hamiltonian" class. . . . . . . . . . . . . . . . . . . . . . . 74

3.11 Cartesian class. . . . . . . . . . . . . . . . . . 75

3.12 Example code: QFISHBOWL. . . . . . . . . . . . . . . 77

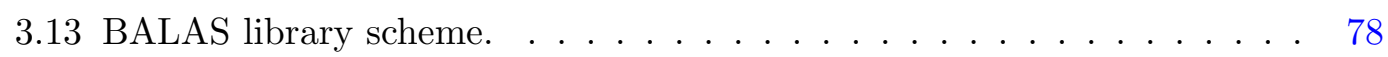

3.14 QSPHERE library scheme. . . . . . . . . . . . . . . 81

4.1 Optical SPIDER scheme. . . . . . . . . . . . . . . . 87

4.2 Structural dipole transition matrix element. . . . . . . . . . . . . . 90

4.3 Attosecond electron wavepacket. . . . . . . . . . . . . . . . . . . . . . . . . . . . . . 92

4.4 Attosecond EWPs interferences. . . . . . . . . . . . . . . . . . . 94 


\section{LIST OF FIGURES}

4.5 Photoionization in presence of an IR laser. . . . . . . . . . . . . . . 98

4.6 Attosecond EWP interferometry in presence of an IR laser. . . . . . . . 101

4.7 General idea of the QSPIDER technique. . . . . . . . . . . . . . . . 104

4.8 Basic QSPIDER algorithm. . . . . . . . . . . . . . . . 106

4.9 Complex dipole structure for the $1 s$ and $2 s$ of $\mathrm{He}^{+} \ldots \ldots \ldots$. . . . 108

4.10 Streaked interferogram in case of $1 s$ of $\mathrm{He}^{+}$: SFA model. . . . . . . . . 110

4.11 QSPIDER retrieval for the $1 s$ of $\mathrm{He}^{+}$: SFA model. . . . . . . . . . . . 111

4.12 QSPIDER momentum interferogram for the $2 s$ of $\mathrm{He}^{+}$: SFA model. . . 113

4.13 QSPIDER for the $2 s$ of $\mathrm{He}^{+}$: SFA model. . . . . . . . . . . . . . 114

4.14 QSPIDER for the $2 s$ of the $\mathrm{He}^{+}$: SFA model. . . . . . . . . . . . 115

4.15 1D Potentials, bound states, and complex dipoles. . . . . . . . . . . 116

4.16 QSPIDER for the ground state of a Yukawa potential: 1D TDSE model. 118

4.17 QSPIDER for the ground state of a Coulomb potential: 1D TDSE model.120

4.18 Lateral shearing interferometry technique in the Optics. . . . . . . . . . 122

4.19 Lateral momentum-shearing for the EWPs interferometry scheme. . . . 124

4.202 D EWPs interferometry. . . . . . . . . . . . . . . . . 127

4.21 2D EWPs lateral momentum-shearing along $y$-direction. . . . . . . . . . 129

4.22 Complex dipole for the $2 s$ and $2 p$ states. . . . . . . . . . . . . 133

4.23 QSPIDER for the lateral momentum-shearing: EWP and 2p-dipole. . . 134

4.24 QSPIDER for the lateral momentum-shearing: EWP and $2 s$-dipole. . . 136

4.25 Yukawa potential and $2 p$ orbital. . . . . . . . . . . . . . 138

4.26 EWP amplitude and phase: exact 2D TDSE calculations. . . . . . . . . 139

4.27 EWPs momentum interferogram: 2D TDSE calculations. . . . . . . . . 141

4.28 QSPIDER for the $2 p_{y}$ of a Yukawa potential: 2D TDSE model. . . . . . 142

5.1 Conceptual Wigner Time. . . . . . . . . . . . . . . . . . . . . . . . 148

5.2 1D Yukawa, Coulomb potentials and ground states. . . . . . . . . . 150

5.3 Dipole matrix element structure for Yukawa and Coulomb potentials. . . 151

5.4 Quantum and classical pictures of the Wigner time delay. . . . . . . . 152

5.5 Advanced and delayed streaking time scheme. . . . . . . . . . . . . 155

5.6 Fourier analysis for the extraction of the streaking time delay. . . . . . . 156

5.7 Streaking traces and time delay in photoemission: Yukawa potential. . 157

5.8 Streaking traces and time delay in photoemission: H Coulomb potential. 158

5.9 Streaking time delay in the short-range and long-range potentials. . . . 159

5.10 Streaking time delay in photoemission for a long-range potential. . . . . 160

5.11 1D Asymmetric model for the carbon monoxide $(\mathrm{CO})$. . . . . . . . . . 162 
5.12 Asymmetric CO molecular dipole transition matrix element. . . . . . . . 163

5.13 Stereo Wigner time delay. . . . . . . . . . . . . . . . . . . . . 164

5.14 Scheme for the stereo TOF. . . . . . . . . . . . . . . . 165

5.15 Left and right semiclassical trajectories. . . . . . . . . . . . . 167

5.16 Extrapolation and TOF methods to extract stereo Wigner time delay. . 169

5.17 CO stereo time delay in photoemission. . . . . . . . . . . . . . . 170

5.18 Comparison of the streaking traces from $\mathrm{H}$ and $\mathrm{CO}$ systems. . . . . . . 172

5.19 Streaking trace by PW and SW projections for the CO molecule. . . . . 173

5.20 Stereo streaking time delay for the molecular case. . . . . . . . . . . . 174

5.21 Analysis of the stereo streaking time delay. . . . . . . . . . . . . . . 175

5.22 Oriented asymmetric molecular system in 3D . . . . . . . . . . . 178

5.23 Molecular attosecond XUV electron wavepacket in 3D. . . . . . . . . . 180

5.24 Stereo Winger time delay by using the TOF method: 3D results. . . . . 181

5.25 Attosecond streaking effect obtained by 3D TDSE. . . . . . . . . . . . . 184

5.26 Streaking traces: 3D TDSE calculations for the HOMO. . . . . . . . . 185

5.27 Streaking traces for the HOMO-1. . . . . . . . . . . . . . . . . 188

5.28 Coherent superposition induced by the IR laser field. . . . . . . . . . . 190

5.29 One photon absorption by an asymmetric molecular potential. . . . . . 190

5.30 Stereo streaking time delay versus the peak intensity of the IR laser field. 191

5.31 Time delay asymmetry on the streaking trace. . . . . . . . . . . . . . . 193

5.32 Asymmetry and scan on the peak intensity of the IR laser field. . . . . . 194

5.33 Asymmetry amplitude and excited population. . . . . . . . . . . . 195

A.1 Extrapolation method and the delay in photoemission. . . . . . . . . . . 203

A.2 Delay in photoemission from a short-range Yukawa potential. . . . . . . 206

A.3 Delay in photoemission from a long-range Coulomb potential. . . . . . . 208 


\section{Introduction}

The physical description of natural phenomena depends on their spatial and temporal scales. For example, the orbital period of the earth around the sun is one year (about $\left.1.3 \times 10^{6} \mathrm{~s}\right)$, the period orbits of the moon around the earth is one month $\left(\sim 9.8 \times 10^{4} \mathrm{~s}\right)$ and the period of the heart palpitations is $\sim 1 \mathrm{~s}$. These phenomena can be considered within the human time scale and can be studied with available technologies.

Faster natural phenomena such as the flapping of bee wings or the vibration period of the strings of a violin $\left(\sim 10^{-3} \mathrm{~s}(\mathrm{~ms})\right)$, can be characterized if a suitable instrument is used, i.e., a camera with a shutter speed of about $10^{-4} \mathrm{~s}$. The typical biological processes of life are in the range of $\sim 10^{-6} \mathrm{~s}(\mu \mathrm{s})$, i.e., the RNA molecule motion or deformation, protein motion in cells, etc. In electronic devices such as a microprocessor, the processing of data occurs in times of the order $10^{-9} \mathrm{~s}$ (ns) (see, for instance Fig. 1.1). Changes in the nuclear structure of molecules, such as the vibrations or the breaking and formation of chemical bonds occur at the characteristic timescale of a few femtoseconds $\left(\sim 10^{-15} \mathrm{~s}(\mathrm{fs})\right)$ to a few thousand femtoseconds or picoseconds $\left(\sim 10^{-12} \mathrm{~s}\right.$ (ps)) $[1,2,3]$.

Nowadays all of these time scales of biological, chemical and physical phenomena can be addressed due to the remarkable advance of the electronic devices, particularly the laser pulses [3].

The femtosecond and attosecond laser sources are considered to be the main tools for resolving the fast electron motion in matter temporally and spatially down to subÅngstrom $\left(1 \AA=10^{-10} \mathrm{~m}\right)$ resolution $[4,5,6]$.

For atomic physics, the most fundamental process is the electron transition between states. Until recently, it was not possible to observe the "motion" of the electrons, because there were no high-resolving tools available. But, with the advent of the at- 
tosecond $\left(10^{-18} \mathrm{~s}(a \mathrm{~s})\right)$ pulses a new time perspective is opened $[3,7]$.

The femtosecond and attosecond pulses have become one of the main tools to measure the fast electron motion. This thesis is devoted to the study of ultrafast electron phenomena as a result of the attosecond and femtosecond pulses interacting with the atoms and molecules.

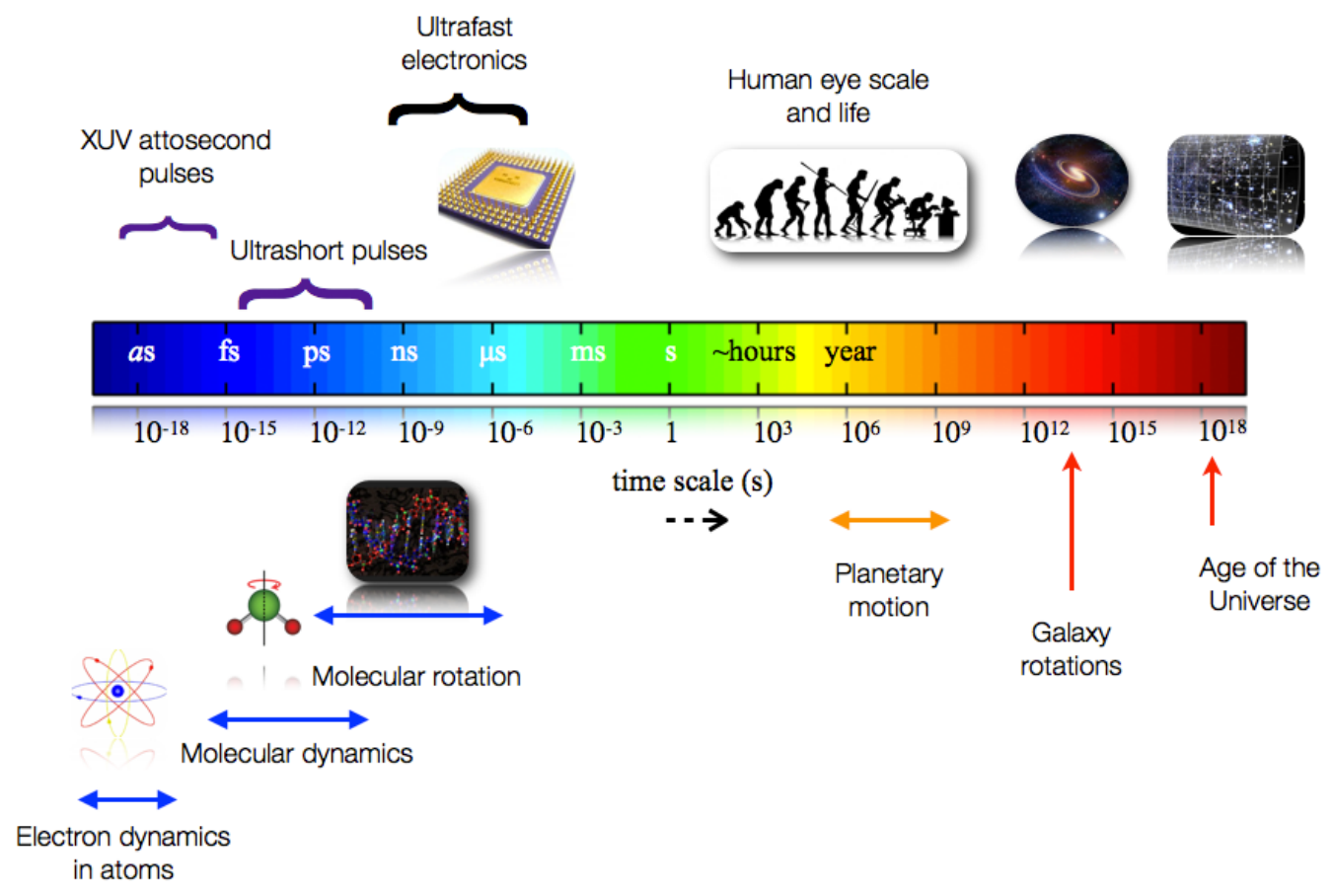

Figure 1.1: Timescale of different physical phenomena. This picture depicts different temporal scales of natural phenomena and technological development. 


\subsection{Brief history of ultra-short laser pulses}

The generation of short laser pulses started right after the first implementation of the laser in 1960 [8] by Maiman. During the following decades, the development of new techniques helped to achieve shorter and shorter pulses [6,9]. A diagram showing the evolution of pulse duration during the 1960-2010 time frame is depicted in Fig. 1.2.

The Q-Switching [10] is an active technique which modulates the cavity losses. When the losses are momentarily reduced the accumulated energy is launched in a short pulse. As the modulation is active, it is limited to the speed of the change in the modulation. This technique is used to generate nanosecond pulses of high energy in solid-state lasers $[10,11]$.

The Kerr Lens Mode-locking (KLM) [9] is a passive technique which achieves two things simultaneously. Using the nonlinear response of a material such as the Kerr effect the modulation of the cavity is much faster leading to very short emissions. To produce short pulses, it is necessary to support a broad spectrum with synchronized phases.

The Mode-locking was reported for the first time by Hargrove et al. in 1964 [12, 13]. The purpose of this technique is to synchronize or "lock" the relative phases of the modes oscillating inside the laser cavity. These frequencies add up coherently (constructively) and the intra-cavity intensity increases.

This technique helped to produce the first femtosecond pulses, typically between $30 \mathrm{fs}$ and $30 \mathrm{ps}$, in extreme cases down to $\sim 5$ fs $[11,14]$.

Femtosecond pulses can only be produced if the gain media in the laser cavity support a broad spectrum. In 1982, a new laser material, the Ti:Sapphire $\left(\mathrm{Ti}_{\mathrm{Al}} \mathrm{Al}_{2} \mathrm{O}_{3}\right)$ was developed by Moulton [15]. This crystal allows the generation of very short pulses because of its large gain bandwidth $(650-1100 \mathrm{~nm})$.

Another breakthrough was developed by Strickland and Mourou in 1985. It is CPA [19], which enables the amplification of femtosecond lasers by several orders of magnitude (from the nanojoule (nJ) to the multi-Joule level).

The CPA technique solves the problem of damage threshold on the crystal when a femtosecond pulse is amplified in the laser. To bypass this problem the ultrashort pulses are stretched out in time, reducing the peak power (typically a $10^{4}$ or higher factor), avoiding crystal damage. Once the pulses are amplified, they are compressed back to the original duration achieving a high peak power. With this process very high peak powers as large as $1 \times 10^{15} \mathrm{~W}$ can be achieved.

Simultaneous to the development of these techniques it was necessary to develop 


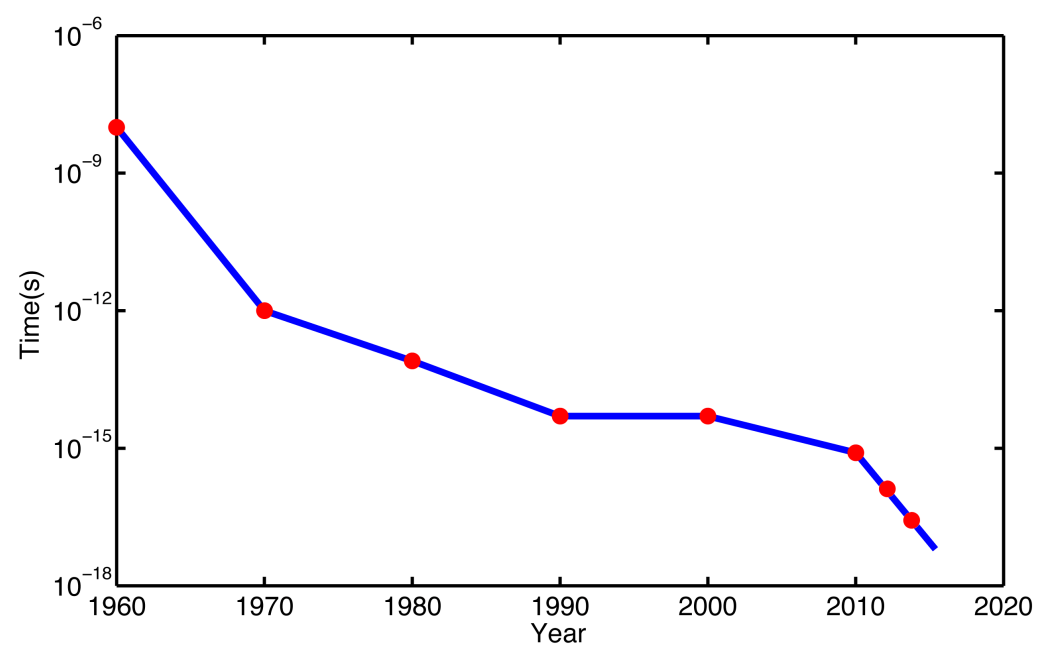

Figure 1.2: Evolution of ultrafast laser pulses. The time duration of laser pulses decreases continually from the discovery of Q-Switching and mode-locking in 1963-64 until 1987 when 6 fs pulses were reported [16]. At this time duration of the laser pulses, 6 fs (three periods of light in the mid-IR), different technology was required in order to study and control natural phenomena at this time scale. This development took about 15 years. According to Corkum and Krausz [6] nowadays the attosecond technology is providing new tools for science. Thus, it is again opening new fields for real-time measurement, i.e., the electron dynamics in the photoemission process $[17,18]$. 
characterization methods of the time profile of these ultrashort laser pulses. Several techniques to diagnose the spectral amplitude and phase of the laser pulse electric field have been developed within the IR electromagnetic spectral range. Some examples of these are: SPIDER [20, 21], Spatially Encoded Arrangement for SPIDER (SEASPIDER) [22], FROG [23], GRating-Eliminated No-nonsense Observation of Ultrafast Incident Laser Light E-fields (GRENOUILLE) [24], SpatioTemporal Amplitude-andphase Reconstruction by Fourier-transform of Interference Spectra of Highly-complexbeams (STARFISH) [25, 26], amongst others.

After the CPA development, the need to compress pulses up to the diffraction limit arises with the purpose of accessing the ultrafast molecular dynamics. Some of the methods to achieve short laser beam pulses of $30 \mathrm{fs}$, of the Ti:Sapphire laser $(800 \mathrm{~nm}$ of carrier wavelength) are the propagation of the laser beam along a gas, where the nonlinear interaction leads to a broader spectrum (filamentation process and nonlinear process in hollow fibers) $[27,28,29,30]$. These methods have been able to generate the shortest few-fs pulses, corresponding to three, two and even shorter, one cycles ( $2.6 \mathrm{fs})$ of the carrier wave frequency [30, 31, 32].

The short laser pulses from oscillators and the high power amplified pulses opened a new research field within the femtosecond timescale. When these pulses are focused close to the diffraction limit about a few $\mu \mathrm{m}^{2}$ at focus, the laser peak intensity can be very high leading to strong field effects.

These high intensities can produce highly nonlinear responses in atoms and molecules. In particular if the intensity of the laser pulse is comparable to the atomic unit $I_{a u}=$ $3.5 \times 10^{16} \mathrm{~W} / \mathrm{cm}^{2}$, the laser field of the pulse will create an electric field comparable to the electrostatic field experimented by the electron inside the atom or molecule.

In such a case, the laser matter interaction is not perturbative anymore and strong field phenomena arise.

The ultrashort laser technology available today allows physicists to address a wide variety of interesting strong field phenomena with unprecedented time-resolution. For example, the tunneling ionization process which leads to the high-order harmonic generation, the above threshold ionization, the double and multi-electron ionization, molecular dissociation and nuclear dynamics, can now be understood and controlled thanks to the ultrashort laser technology [6, 33, 34, 35, 36].

Attosecond science is the most important spin-off of strong field physics. Based on the generation and control of the envelope and carrier of the femtosecond pulses $[37,38]$, it is possible to generate and synthesize attosecond pulses.

Physicists have developed novel pump-probe techniques based on the combined use 
of both attosecond and femtosecond pulses which allows to access to the real-time electron dynamics [39]. Attosecond streaking, is one example of this techniques, which allows us to access to the real-time electron dynamics, i.e., it was used to characterize the electronic Auger relaxation dynamics in krypton [39].

Finally, one of the amazing phenomenon in attosecond physics is the HHG which enables to the synthesis of attosecond pulses [37, 38] within unprecedented short time durations. The next Section will be dedicated to describing the HHG process.

\section{$1.2 \quad$ High-order harmonic generation}

The first experimental demonstration of the emission of high-order harmonics was observed by McPherson et al. [40] using an intense ultraviolet (248 nm) laser pulse and by Ferray et al. [41] with an IR Nd:YAG laser (1064 nm) focused in different atomic gas targets. These experiments, and in particular [41], show a different harmonic shape than expected by the perturbative theory. This harmonic emission had a distinct form, with three regions clearly distinguishable, namely: (i) a strong signal for low harmonics (perturbative region), (ii) the middle region harmonics, larger than the $\sim 5$ th harmonic (plateau region which has a constant amplitude for intermediate harmonics) and (iii) the high harmonic region (cutoff region).

The interaction of these ultrashort IR laser pulses of high intensity $\left(10^{13}-10^{15}\right.$ $\mathrm{W} / \mathrm{cm}^{2}$ ) with a gas targets, leads to the electron being released to the continuum by tunneling and with it to the HHG process. A classical picture describing this phenomenon, was first introduced by Corkum [42] in 1993 using the so-called three-step or simple man's model.

This model suggests that, first, when the electric field of the laser is around its maximum, the atomic potential barrier is deformed in such a way that the electron can be ionized via tunneling. It is supposed that when the electron appears in the continuum, it has a position coordinate and velocity equal to zero. Second, once the electron is in the continuum, it is accelerated by the driven laser electric field gaining energy, as it is illustrated in Fig 1.3. Once in the continuum, the influence of the Coulomb potential of the remaining ion is neglected. Third, when the electromagnetic field of the laser changes its sign, the electron turns back to its parent ion and returns to its initial ground state (recombination), converting its kinetic energy in a high energy photon of frequency $\omega_{\max }$. This process occurs at some time around the zero of the laser electric field (see part-(v) in Fig. 1.3). Then, the atom emits its excess energy in the form of XUV radiation. Fig. 1.3 illustrates the classical picture of the HHG process. 
According to this classical model the maximum photon-emission energy $\omega_{\max }$ is given by $\omega_{\max }=I_{p}+3.17 \mathrm{U}_{p}$, where $I_{p}$ is the ionization potential of the system, and $\mathrm{U}_{p}=\frac{\varepsilon_{0}^{2}}{4 \omega_{0}^{2}}$ is the the ponderomotive energy. Here, $\varepsilon_{0}$ and $\omega_{0}$ denote the peak amplitude of the IR oscillating electric field and its carrier frequency, respectively ${ }^{1}$.

Another theoretical approach, with grounds on Quantum Mechanics, was been developed by Lewenstein et al. in 1994 [43]. This theoretical approximation is usually referred as Lewenstein's model or SFA. In this scheme the TDSE is solved under three main assumptions: (i) the contribution to the evolution of the system of all the bound states can be neglected, but not that of the ground state one; (ii) The depletion of the ground state is neglected and (iii) in the continuum, the electron can be treated as a free particle moving in the laser electric field and is not affected by the atomic potential.

Within this approximation, the HHG process can be easily characterized from a quantum viewpoint. There are several appealing facts about this model. The odd harmonic structure, the plateau spectral region and the cutoff law energy naturally are reproduced. It is also demonstrated that the electron short-long trajectories emerge directly in the description of the HHG process using SFA. This model has been used quite successfully, in particular, for the analysis of the attosecond synchronization of high harmonics [44].

However, one of the main limitations of this model arises by considering how the continuum electron recombines when it is near the parent ion. Here, neglecting the electron-potential interaction is rather questionable [45, 46]. Various efforts have been made to improve upon the SFA model, i.e., by including the Coulomb distortion [45, $47,48,49]$.

The most general treatment of the strong field interaction with matter is the solution of the TDSE and in particular it can be applied to HHG. Thus, it is interesting to point out that the full time-space position electron density depicted in Fig. 1.4a), shows a similar behaviour described by the three steps classical model.

Fig 1.4 depicts an example of HHG spectra according to the 1D TDSE numerical solution. Fig. 1.4a) shows the electron $z$-position distribution as a function of time and in Fig. 1.4b), we depict the corresponding HHG spectrum. The electron density is obtained as a result of the interaction of a strong IR laser field with a hydrogen atomic 1D model. The IR laser can be considered as a Titanium Sapphire (Ti:Sa) laser with central wavelength of $800 \mathrm{~nm}$ ( $\omega_{0}=0.057$ a.u. of central frequency) and a time duration

\footnotetext{
${ }^{1}$ Note that atomic units are used throughout this thesis. This means that the fundamental constants are $\left|q_{e}\right|=m_{e}=\hbar=a_{0}=1, c=137$, etc.
} 


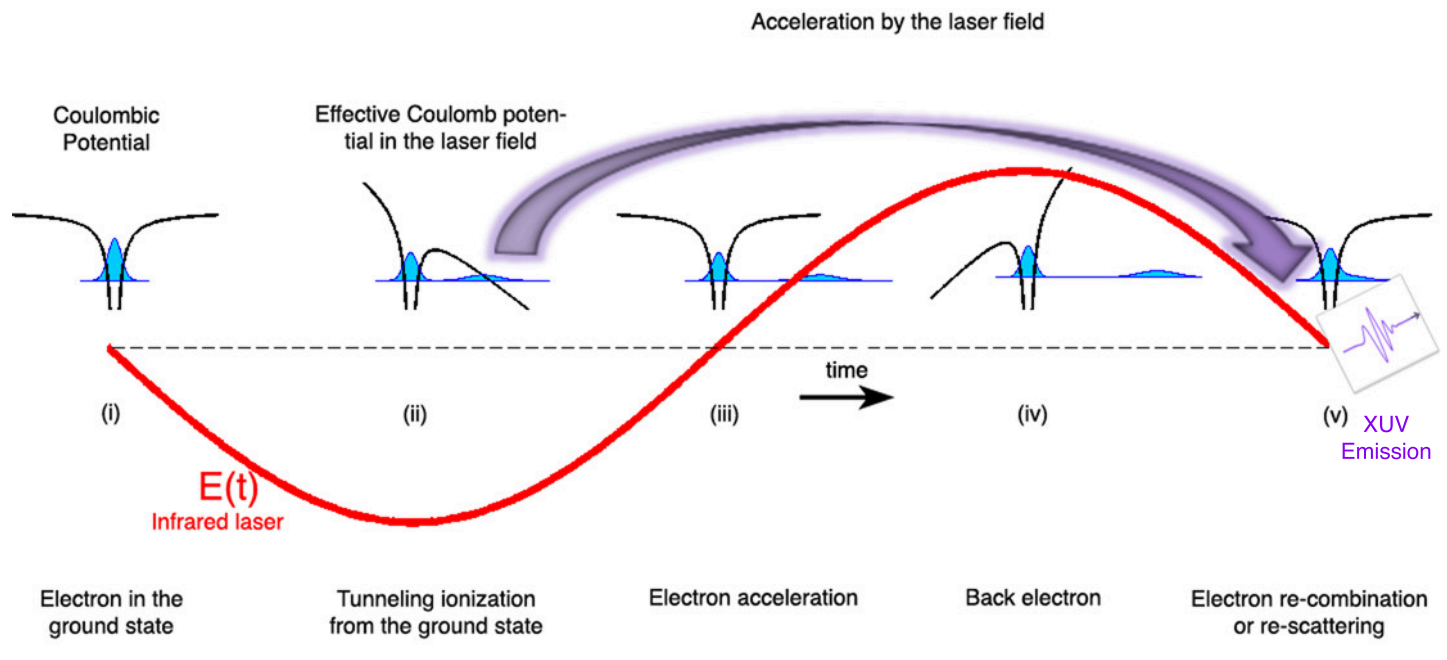

Figure 1.3: Classical scheme of HHG process. This picture describes the classical mechanism of the HHG and with it the generation of attosecond pulses. (i) The Coulombic potential and the electron in the ground state, when the laser is turned off. (ii) When the IR laser electric field reaches the negative maximum, the Coulombic potential is deformed creating a potential barrier. Thus, the electron tunnels out through the potential barrier. The electron is accelerated in the (iii) and (iv) by the driving laser electric field in such a way that the it gains energy and goes back to the ion core between (iv) and (v). (v) The electron re-collides with its parent ion, then it re-combines with the atom emitting the gained energy in form of a high order harmonic frequency of the fundamental laser or XUV frequency photon. 
about 13-fs at FWHM. It is clear that the three steps described in Fig. 1.3, namely tunnel ionization, movement in the continuum and recombination, can be appreciated within the time-position map of the electronic current density.
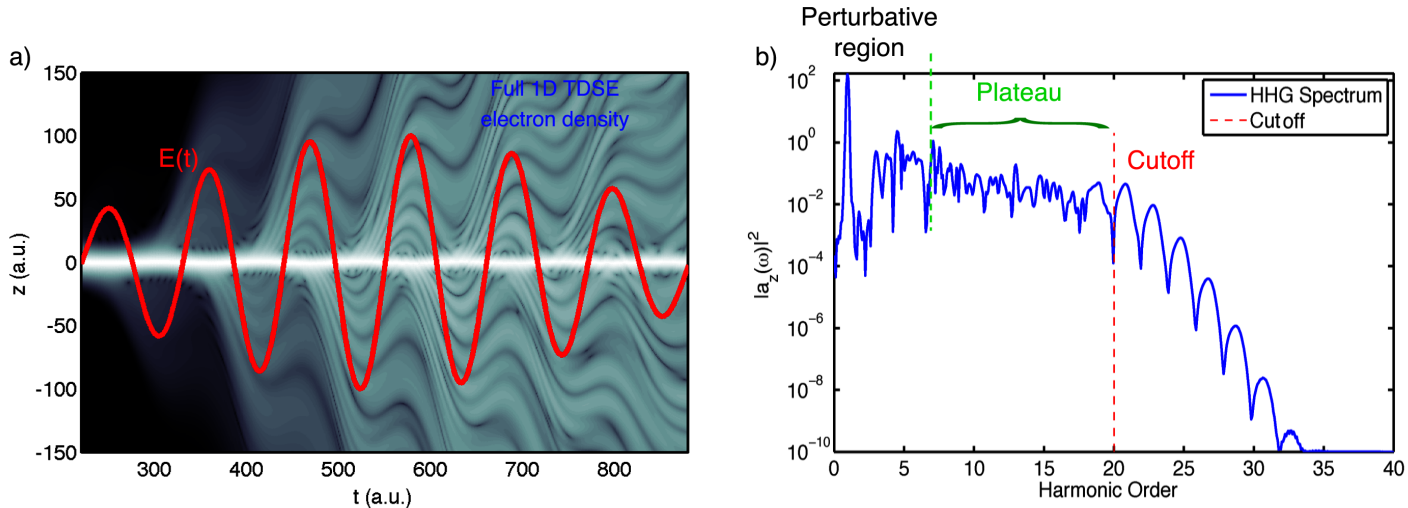

Figure 1.4: Strong field ionization and HHG spectrum. a) TDSE numerical solution for the $z$-position probability density of the electronic wavefunction as a function of time (gray color scale, black is the lowest and white, the highest). This time-space electron density is the result of the strong IR laser field (red solid line) interaction with the 1D hydrogen atom model. The peak intensity of the IR laser is $8.5 \times 10^{13} \mathrm{~W} / \mathrm{cm}^{2}$. The simulation is performed with a $\sin ^{2}$ envelope of 10 cycles. b) HHG spectrum as a function of the harmonic order is depicted in the blue line. The spectral emission radiation is defined as the absolute square of the dipole acceleration Fourier Transform along the time axis. The red dashed vertical line denotes the cutoff photon energy $\omega_{\max } / \omega_{0}$. The harmonic spectrum consists of three main parts, the perturbative, the plateau and the cut-off spectral regions. First, the perturbative region denotes the spectral range where the perturbative theory describes perfectly the harmonic emission behaviour. Second, the plateau is the range where the harmonic intensity seems to be constant. And third, when a drastic reduction of the harmonic intensity is reached, the cut-off region is defined. This region shows that the short-long trajectories correspond to "single" recombination events.

In addition, note that the emitted HHG spectrum (Fig. 1.4b)) is composed of odd harmonics only. It is so because the time-space symmetry of the atomic and laser potential cause the radiated dipole acceleration to follow the negative value of the electric field [50]. These harmonics are integer multiples of the fundamental IR laser frequency $\omega_{0}=0.057$ a.u.

In the next Section we will review briefly the synthesis of the HHG spectra in an APT and a SAP. 


\subsection{Attosecond pulse generation}

One of the remarkable features of the HHG is that the emitted radiation is a coherent. As the re-collisions are periodic, these harmonics are emitted at each zero of the field in short bursts of light. Because of the coherence it produces a short pulse and if there are several re-collisions provoked by the driving IR laser field, then the emission is a train of pulses.

Many experimental efforts have been done to control and manipulate the coherent XUV light from HHG [37, 38, 51, 52], to produce attosecond pulse trains [38] and a single attosecond pulse [37].

A train of pulses is generated by filtering the higher frequency part of the HHG spectrum that corresponds to the plateau region [38]. The first experimental observation of an APT was obtained by Paul and coworkers [38] by isolating the five consecutive emitted harmonics from Ar. They measured an APT with a duration per pulse of 250 as, separated by a one half-cycle of the IR laser period with the train extending over some $10 \mathrm{fs}$.

To achieve single attosecond pulses many techniques exist $[37,53,54,55]$. By manipulating the temporal duration and the shape of the electric field the number of re-collisions can be controlled.

The fundamental process to generate an APT consists of isolating a spectral region of the HHG spectrum. Experimentally, spectral filtering is achieved using different materials to remove the lowers harmonic [38].

Figure 1.5 depicts a numerical calculation performed with the Lewenstein's model. The plot a) shows the selected spectral region between the plateau and cutoff (see Fig. 1.5a)). Then, by Fourier Transform of the selected signal, the APT can be generated. This process also can be performed on the plateau region as it is shown in reference [56].

With a similar procedure to the one described in Fig. 1.6, Hentschel et al. [37] reported the first experimental observation of an isolated SAP. The measured pulse had a time duration of about 650 as. Such a measurement was performed by filtering a few cut-off harmonics. The HHG spectrum had been produced by focusing a linearly polarized laser pulse, with a wavelength of $750 \mathrm{~nm}$, a time-duration 4-fs and stabilized $\mathrm{CEP}$, in Ar.

Fig. 1.6 depicts the same process followed in Fig. 1.5, but now to obtain a single attosecond pulse. In this case, the physical mechanism for the HHG differs that in the APT generation. The driving IR laser field is a few-cycle pulse. According to the semiclassical or Lewenstein's model, this means that few re-collision events will take 

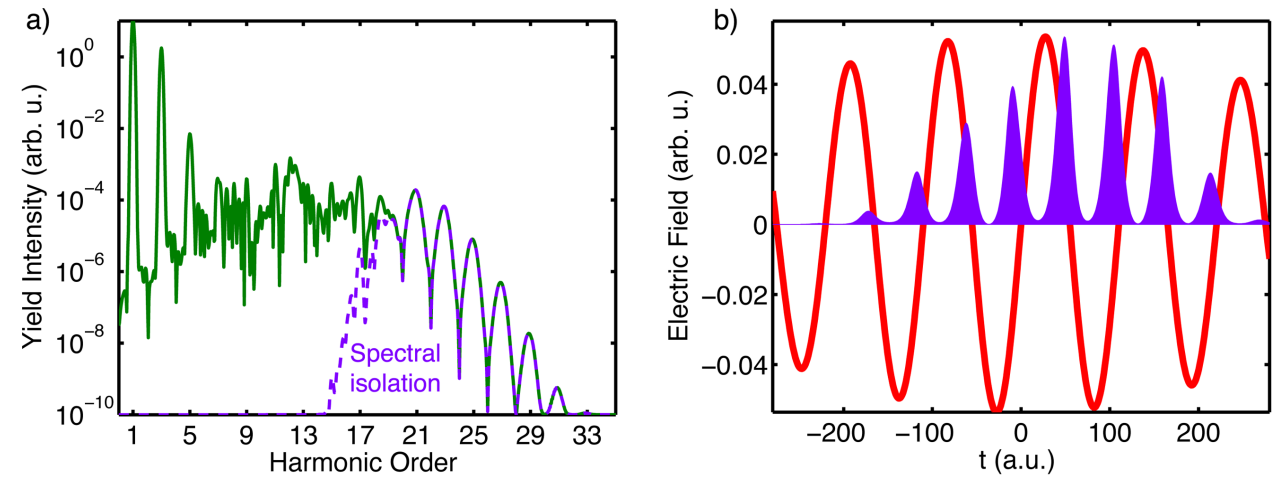

Figure 1.5: Attosecond pulses train. a) In green dark line, the HHG spectrum as a function of the harmonic order which is computed by the SFA model for the hydrogen atom. The isolated spectrum between the plateau and cut-off region is marked in a violet dashed line. b) In violet area, the attosecond pulse train intensity as a function of the time. This APT is obtained by computing FFT of the isolated spectrum of a). The time domain of the IR laser field is shown by the red line. The IR laser peak intensity utilized for such a calculation is $1 \times 10^{14} \mathrm{~W} / \mathrm{cm}^{2}$ and the FWHM $=18$-fs, the central frequency is $\omega_{0}=0.057$ a.u. $(800 \mathrm{~nm}$ of wavelength).

place. Then, it is expected that the control of a single re-collision event leads to the emission of a SAP.

Another interesting method to isolate SAP, was developed by Sola et al. [53]. By modulating the polarization of a carrier-envelope phase-stabilized short laser pulse, a very precise control the EWP dynamics can be achieved. Then, they used high-order harmonic generation to probe these dynamics. This scheme temporally confines the XUV emission to an SAP with a broad and tunable bandwidth [53].

These XUV sources, the APT and the SAP, need to be characterized in time by measuring the electric field amplitude and phase. This is a very hard task because these pulses in the UV and XUV are absorbed by the materials. In addition, it is very difficult to produce a nonlinear response in this spectral range. For these reasons, the manipulation of the HHG spectra in the lab to perform a diagnostic of the XUV source is difficult.

Nevertheless, the characterization of these pulses is possible. By analyzing the emitted electrons which are created by the interaction of the HHG signal with gasses several techniques have been developed. Most of the measurement techniques take advantage of the high-photon energy of the APT (or the SAP) and combine it with a moderated intense IR laser to modulate the ionization dynamics. Thus, the temporal characteristics of the pulses are inferred.

These are the basis of the RABBIT technique $[38,57]$ to diagnose an APT, and the 
a)

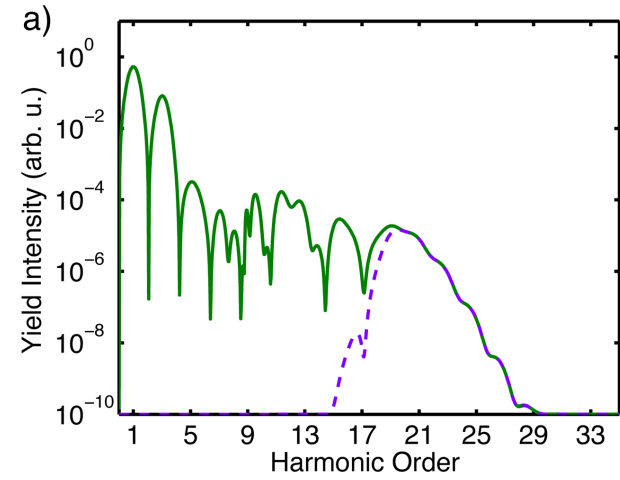

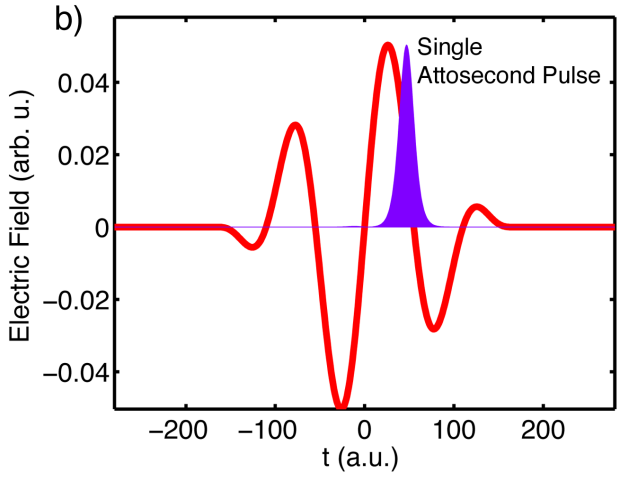

Figure 1.6: Single attosecond pulse. a) and b) depict the same that in Fig. 1.5 but the HHG spectrum is driving by few-cycle IR laser as it is denoted by the red line in b). A single attosecond pulse is obtained when the same filter employed in Fig. 1.5a) is utilized to the spectrum in a).

FROG-CRAB technique $[58,59]$ to characterize a SAP $[59,60]$. Both techniques provide a full reconstruction of the temporal shapes of an APT [38] and a SAP [58, 61], respectively.

The FROG CRAB technique uses the attosecond streak camera [37, 59, 61], which is a very good technique to characterize the isolated XUV attosecond pulse and the ultrashort IR laser field.

The attosecond streak camera is a pump probe technique, where the time dependence of the laser can be mapped into the final momentum of an electron. Electrons ionized by the SAP in the presence of a moderated IR laser field are shifted or streaked in momentum by the value of the vector potential at the ionization time [59, 61]..

The final electron energy or momentum depends on the time delay between the isolated XUV SAP and the oscillating IR laser field. In the Chapters 4 and 5 we will address in detail the theoretical aspects of this revolutionary technique.

More theoretical techniques have been proposed later on, i.e., XSPIDER [62], which is an SPIDER version for the characterization of a single XUV attosecond pulse. However, we will not comment the details about this wonderful XUV optics metrology field in this thesis.

The generation and control of the attosecond pulses, and the related diagnostic techniques are the main tools of the attoscience and open new possibilities to study ultrafast dynamics.

Next we provide a brief review of the most relevant experimental, theoretical efforts and the state-of-the-art in the attoscience. 


\subsection{State-of-the-art in attosecond physics}

Electron structural and dynamics information can be experimentally scrutinized by utilizing the now available attosecond technology. The key point is how this information can be deducted from the results of laser-matter interaction. This is one of the big challenges for attosecond science.

During the last two decades, many experimental and theoretical efforts have been done to develop and understand the attosecond tools and their main mechanisms, e.g., the high-order harmonic spectroscopy and the attosecond spectroscopy [6, 63] .

According to Corkum and Krausz in [6] three main areas have been opened with attosecond science: metrology of the attosecond pulses [64], attosecond spectroscopy and attosecond dynamics. Several experiments have been carried out within these fields. Here, we will address only a few of those regarding the electron structure and attosecond electron dynamics. We restrict ourselves to these last topic because our interest is to access to the structural and dynamics information of the system.

\section{Electron structural information}

In 2004 Itatani et al. reported one of the first experiments which utilized the high-order harmonic spectroscopy to extract structural information. They performed a tomography reconstruction of the HOMO of the $\mathrm{N}_{2}$ [5]. According to Itatani's paper the dipole matrix element of the HHG process contains structural information about the initial state of the electron inside the molecule.

This tomography method consists of three steps: (i) the measurement of the HHG spectrum as a function of the molecular alignment angle between the IR laser, linearly polarized, and the molecular axis, (ii) the measurement of the harmonic emission of an atomic reference system with similar ionization potential than the $\mathrm{N}_{2}$ molecule, i.e., the Ar atom, and (iii) the application of a reconstruction algorithm for all the different angles.

To perform a full tomography reconstruction, the phase measurement is important in the Fourier analysis required for the retrieval as well. In spite of the fact the authors in [5] reconstructed the HOMO for $\mathrm{N}_{2}$, the relative phase of the harmonic emission was not measured for every alignment angle.

Haessler et al. [65], addressed this problem and measured the phase of the HHG spectrum for each alignment angle in both systems, i.e., the atomic reference and the molecule under study. They took advantage of the RABBIT technique to measure the spectral phase for every HHG signal. In this way, a full experimental reconstruction of 


\section{STATE-OF-THE-ART IN ATTOSECOND PHYSICS}

the HOMO and HOMO-1 for the aligned molecule $\mathrm{N}_{2}$ was performed in 2010.

Even though, the high-order harmonic spectroscopy appears to be a good technique to image the molecular orbital, this method requires several experimental steps. Even more, the difficulties to find an atomic reference potential which matches the ionization potential of the molecular system in study, reduces drastically the range of molecules which can be tackled.

In particular, other interesting developed experiment to extract structural information was reported by Remetter et al. [66] in 2006. Such experiment consists of making use of the ionization led by the interaction of an APT in the presence of a weak IR laser with an atomic gas target, e.g. Ar. The multiple EWPs created by the APT were measured onto a final momentum interferogram, which was clearly the function of the time delay between the APT and the IR laser pulse. It is remarkable that this final interferogram encodes structural nodal information about the bound state of the Ar system. However, an algorithm to retrieve the full EWP phase or the bound-free dipole transition matrix element, is still absent.

Here we introduce two of the main research questions of this thesis: first, is it possible to design a new technique to extract structural information, i.e., bound-free dipole transition matrix element, by applying the attosecond science tools? Second, can this technique be a single shot and an auto-referenced? These questions will be addressed with more details in the Chapter 4 .

\section{Electron dynamics information}

Furthermore, when a SAP or an APT interacts with an atom or a molecule, a coherent ultra broadband EWP is created. If the photon energies of the attosecond pulse are higher than the ionization potential, the electron is then ionized and the momentum distribution of these electrons maps the characteristics of the attosecond pulse and the parent system [61, 67].

The emission of this EWP is not an instantaneous process in the sense that a short period of time is taken by the electron to leave from the binding potential [68]. This delay in the photoelectron emission process is known as Wigner time delay or WignerEisenbud-Smith time [69, 70]. Formally, the Wigner time is defined as the energy derivative of the dipole phase or the phase shift that takes the EWP in the one photon ionization process $[69,70,71]$.

Utilizing the attosecond streaking technique Schultze et al. [17] have measured the relative delay in photoemission of Ne from the $2 s$ and $2 p$ states [37]. The measurement is based on the production and control of a SAP of around 200 as duration and central 
energy of $106 \mathrm{eV}$ joint with an ultrashort IR laser pulse. The results showed a 21 as relative delay between both emission channels $(2 s$ and $2 p)$ whereas the Wigner-time theoretically calculated given in the same paper predicted a value of $6.4 \mathrm{as}$.

Recently, the RABBIT technique [38] has been employed to measure the relative delay between the $3 s$ and $3 p$ states in Ar [18]. This technique uses an APT with mean energy of $35 \mathrm{eV}$ in the presence of a moderate IR laser pulse. In this case the $3 p$ electron shows a delay of around 100 as with the $3 s$ electron which seems to be released early by the laser field. In the same paper, they have found that an extra delay is introduced in the measurement process seeming like an additional mechanism is involved in.

This disagreement between theory and experiment has attracted the attention of several experts. The aim is to explore new theoretical proposals to find a clear physical explanation of the experimental results. The large measured time delay in photoemission suggests that the Wigner time is not sufficient to explain the streaking or experimental results and an additional mechanism seems to be implicated.

Pioneering works by Nagele [72], Kheifets [73], and Ivanov [74], have found that the IR laser field might be responsible for the large delay observed in the experiment. In particular, Ivanov and Smirnova suggested that the measurement is influenced by the coupling between the IR laser pulse and the Coulomb potential of the system [74].

Recent work has also theoretically addressed the time delay in small molecules, such as hydrogen molecules [75, 76], emphasizing the consequences to have two centers in the angular dependence on the Wigner time. In reference [76] they have also studied the dependence of the Wigner delay on the internuclear distance and the angular alignment of the hydrogen molecular ion. These results show that the Wigner time delay also contains information about the geometrical structure of the molecule. Furthermore, and most importantly, from the observable streaking delay the intrinsic Wigner time can be extracted. However, it is important to note that the Coulomb-laser coupling can not be removed from the measurement process.

Those studies about the influence of the IR laser on the detection of the Wigner time have shown that the Coulomb-laser coupling plays an important role in the measurement process.

Pioneer theoretical studies by Maquet et al. [68] suggest that the Coulomb tail, i.e., $-Z / r$, and its coupling with the IR, adds up a symmetry influence to the detection of the Wigner time $[72,74]$. This means that the CLC is symmetric regarding to the potential well, in the sense that the electrons emitted on the left and right experiments the same CLC delay in the streaking measurement process.

Ones would wonder if an alternative method would remove this CLC influence [77]. 
It is by comparing the extracted left-right with respect to the scattering center (core of the potential) time delay by the attosecond streak camera that the CLC is removed from the measure. However, in case of symmetric potentials, i.e., hydrogen, this asymmetric delay will remove the CLC as well as the Wigner time delay.

Throughout this thesis we also address another research questions regarding to the electron dynamics within the photoemission process. We wonder if an alternative method can be used in the detection of the delay in photoemission that directly relates the Wigner time delay with the streaking time delay and, simultaneously, if this method removes the so-called Coulomb-laser coupling from the measurement process. These questions will be studied with more details in the Chapter 5 .

\subsection{Aim of the thesis}

With the motivations cited above, the aim of this thesis is to apply the attosecond science tools with the goal to extract two different kinds of information, namely: electron structural information and electron dynamics information in the photo-ionization process assisted by an IR laser pulse.

In particular:

(i) On the one hand, we will propose a general technique to reconstruct the complex bound-free dipole transition matrix element, by employing an interferometric method. As in Optical Physics, where there exists a set of interferometric techniques to retrieve the amplitude and phase information of the laser beam, we will construct an analogy for the quantum physics. But now these analogies will be applied to the reconstruction of the attosecond EWPs. By SFA analytical and TDSE numerical calculations, we will demonstrate that the subsequent application of two delayed attosecond pulses, in presence of a weak IR laser field, leads to the emission of two EWPs to continuum. If the central frequency of the XUV attosecond pulses are the same, those EWPs will interfere in the momentum space. The resulting interferogram then contains full information about the dipole phase difference between both ionization events. We will show that this scheme is conceptually equivalent to the so-called SPIDER interferogram in Optics.

(ii) On the another hand, dynamics information, such as the Wigner time delay, will be studied by utilizing the attosecond streaking technique with an alternative proposal to measure the delay in the photoemission process. Based on the TDSE numerical solution in 1D and 3D for an small asymmetry molecular system, we will show that the stereo Wigner time delay can be directly associated to the stereo streaking time 
delay. The latter will be obtained by the calculation of two streaking traces, one on the left and another on the right with respect to the ion core (or linearly polarized laser field). The great advantage of this stereo technique is that the problematic CLC will be removed from the measurement process.

\subsection{Outline}

The outline of this thesis is as follows. In Chapter 2, an overview of the theoretical tools the Quantum Mechanics employed to the strong field processes is addressed. Mainly the Schrödinger formalism and the Strong Field Approximation approach are described in this second chapter. In addition, we will describe the numerical methods to implement the TDSE in different geometries, i.e, the cartesian coordinates in 1D, 2D, and 3D, and the cylindrical coordinates for a full 3D calculation that will be required to address adequately our research questions.

In the following Chapter 3, we will define the computational tools that we have developed to perform the strong field interaction of laser pulses with matter, and the designed strategy to address, from a computational viewpoint, our physical problems.

In Chapter 4, we will study how the structural information, i.e., the bound-free dipole transition matrix element, can be retrieved by employing attosecond science tools. We will do analogies between optical techniques to characterize the ultrafast laser pulse and the quantum EWP created by the attosecond pulse interaction with an atomic or molecular system.

In the next Chapter 5, the study of the Wigner time delay in photoemission will be addressed. We will propose here a novel method to remove the induced CLC in the detection process of the fundamental Wigner time delay.

Finally, we will present the conclusions and a brief outlook. We will also include futures studies or open research questions to be considered in the near future. 
OUTLINE 


\section{Theoretical and numerical methods}

\subsection{Strong field interaction}

At the atomic time and space scales, the electron structure and electron dynamics description of the matter is fully governed by the laws of Quantum Mechanics. Typically, the study of the Quantum phenomena has been performed within the frame of the perturbative theory. It is a very good approximation in case that the coupling interaction potential of the system with an external field can be considered as a perturbation of the field-free Hamiltonian [78, 79, 80, 81]. This theoretical approach has been quite successful to explain an ample range of experimental results [82, 83, 84]. However, in case of ultrashort and ultra intense laser pulses, the interaction term can not be treated any more as a perturbation of the field-free Hamiltonian. A new and totally different theory needs to be developed in order to explain the experimental results in this regime.

The most general treatment of the Quantum Mechanics is the Time Dependent Schrödinger Equation. It governs the time evolution of a wavefunction and with it, a quantification of the observables can be obtained.

Pioneering theoretical approaches have been developed by Keldysh [78], Faisal [79], Resnik [80], Lewenstein [43], etc., to describe the main processes and their mechanisms of the referred Strong Field Interaction phenomena [85]. By utilizing the S-Matrix, interesting phenomena, such as multi-photon ionization, tunneling ionization, HHG, ATI, etc., have been successfully explained under some ingenious assumptions.

In this Chapter we will describe the theoretical tools required to address the physical problems and questions that this thesis proposes to answer. 


\section{STRONG FIELD INTERACTION}

Two main formalisms are addressed: first, the Schrödinger formalism, where the time dependent quantum evolution of a wavefunction by the TDSE is used to compute the observables of interest. Second, the Chapter will describe the Strong Field Approximation as a tool to study the ionization led by attosecond pulses assisted by a moderated IR laser field.

Furthermore, another important goal is to set up our numerical methods to solve the TDSE which will be used throughout the rest of this thesis.

The organization of this Chapter will be as follows. In the Section 2.1.1, we begin our theoretical approach by defining the general non-relativistic Schrödinger formalism for the description of the electron wavefunction dynamics and its evolution when an general external electromagnetic field is acting on the system. In the following Section 2.1.2 is devoted to a fast review of the time evolution operators as an alternative to solve the TDSE. This scheme considers that initially the wavefunction is well known and the problem is reduced to the time-space propagation of this initial state to a future time. In the Section 2.1.3 we derive the formal form of this evolution operator when the Hamiltonian of the system is time independent. As a natural consequence, in Section 2.1.4, we will review how such an evolution operator changes if the Hamiltonian is time dependent and commutes on different times. The measured observables of the system are also defined in a general way in Section 2.1.5.

In the Section 2.1.6, we describe the general Strong Field Approximation under the time propagation operator formalism. It is also described in Section 2.1.6.1 the Volkov states for a free electron interacting with a strong laser field. Thus, with this theoretical basis, we derive the SFA complex transition amplitude in Section 2.1.6.2 in the length gauge.

In the next Section 2.2 is dedicated to describe the numerical algorithms used to solve the TDSE in two different symmetries or geometries, namely cartesian coordinates, 1D, 2D and 3D, and cylindrical coordinates. In particular, Section 2.2.1 presents how the general evolution operator and the wavefunction evolution are written within the Spectra Split Operator method in cartesian coordinates for the 1D, 2D and 3D cases.

Numerical aspects about position and momentum space grids discretization are defined in Section 2.2.1.2. In addition, the numerical implementation of the Hamiltonian of the system, wavefunction and potential are addressed in Section 2.2.1.3. Section 2.2.1.4 describes how the discrete and continuum spectra are computed by using the reverse imaginary time propagation method, the diagonalization matrix method and the $\mathrm{Nu}$ merov method. In the next Section, 2.2.1.5, we define the laser pulses used throughout 
this thesis. The Section 2.2.1.6 includes brief comments about the used numerical tools to perform the real-time evolution of the system under the influence of the laser pulse, from the initial (bound) state to the final (continuum) one.

In the Section 2.2.1.7 we will give a short description of our numerical absorbing boundary method. The quantum mechanics numerical observables which will be used throughout this thesis are described in Section 2.2.1.8.

In the next Section 2.2.2, we will address the numerical implementation of the TDSE solution in cylindrical coordinates and a similar description of the position-momentum space grids will be given as it was done for cartesian coordinates in Section 2.2.2.2.

Finally, in the Sections 2.2.3.1 and 2.2.3.2 the fourth-order Runge-kutta method will be described in order to explain how the complex transition amplitude for the ionization is computed via SFA theory.

\subsubsection{Schrödinger formalism}

Within non-relativistic Quantum Mechanics, the most general description of an atomic or molecular system and their interactions with an external electromagnetic field $\mathbf{E}(\mathbf{r}, t)$ is given by the state $|\Psi(t)\rangle$ or wavefunction $\Psi(\mathbf{r}, t)$. This wavefunction allows us to predict the electron time-space evolution of the system and encodes complete information about the observables. For example, in case of the electron ionization by an IR strong field laser, the measured common observables are: the photoelectron spectra, the electron momentum distribution, the momentum and energy expectation values, asymmetry of detected electron left-right with respect to the linearly polarized laser, among others [3].

The time dependent Schrödinger equation describes the time-space evolution of the wavefunction. In case of atomic or molecular system under the single active electron approximation, the TDSE reads:

$$
H \Psi(\mathbf{r}, t)=i \frac{\partial}{\partial t} \Psi(\mathbf{r}, t),
$$

where $H$ defines the full Hamiltonian operator of the system and contains all the interactions of the electron with the nucleus and with any arbitrary external field.

The Hamiltonian operator is:

$$
H=T+V_{0}(\mathbf{r})+V_{\mathrm{i}}(\mathbf{r}, t)
$$




\section{SCHRÖDINGER FORMALISM}

where $T=\frac{\mathbf{p}^{2}}{2 \mu}$ denotes the full kinetic operator with $\mathbf{p}=-i \nabla$ is the canonical momentum operator and $\mu=\frac{m_{e} M}{m_{e}+M} \sim m_{e}$ the reduced mass of the system. Here, $M$ is the nucleus mass. The $V_{0}(\mathbf{r})$ is the electrostatic potential energy of the system, i.e., it is defined from the Coulomb interactions, and $V_{\mathrm{i}}(\mathbf{r}, t)$ is the potential energy which defines the electron interaction with the external field. The functional form of the potential $V_{\mathrm{i}}$ will depend on the gauge used to described the electromagnetic field.

In the strong field scientific community, it is well known that two gauges are mainly utilized to describe the interaction of laser with matter, namely: the length gauge and the velocity gauge. Under the dipole approximation the electromagnetic field of the laser $\mathbf{E}(\mathbf{r}, t)$, is considered not space dependent $\mathbf{E}(\mathbf{r}, t) \approx \mathbf{E}(t)$. This assumption is based on the fact that the wavelength $\lambda$ of the laser is larger than the atomic Bohr radius $a_{0}$ $\left(\frac{\lambda}{a_{0}} \gg 1\right)$ [86]. For instance, when a laser beam is focused on a gas target, usually the focal spot is about of few $\mu m^{2}$. Thus, the spatial electric field which experiments each atom of the gas is practically constant. In such a condition, the dipole approximation is a good estimation of the physical process.

The interacting potential in the length, $V_{\mathrm{i}, \mathrm{LG}}$, and velocity, $V_{\mathrm{i}, \mathrm{VG}}$, gauges read:

$$
\begin{aligned}
V_{\mathrm{i}, \mathrm{LG}}(t) & =\mathbf{r} \cdot \mathbf{E}(t), \\
V_{\mathrm{i}, \mathrm{VG}}(t) & =\mathbf{p} \cdot \mathbf{A}(t)+\frac{1}{2} \mathbf{A}^{2}(t),
\end{aligned}
$$

respectively. $\mathbf{E}(t)$ is the laser electric field and its associated vector potential is $\mathbf{A}(t)=$ $-\int^{t} d t^{\prime} \mathbf{E}\left(t^{\prime}\right)$. According to the references $[50,86,87]$ the conversion between these two equations can be easily done it by following the gauge transformation rules:

$$
\begin{aligned}
H_{\mathrm{LG}} & =Q H_{\mathrm{VG}} Q^{-1}-\nabla \Lambda(\mathbf{r}, t), \\
\left|\Psi_{\mathrm{LG}}(t)\right\rangle & =Q\left|\Psi_{\mathrm{VG}}(t)\right\rangle .
\end{aligned}
$$

The above transformation corresponds to a translation $Q=\exp (-i \Lambda)$ of the wavefunction defined by $\Lambda(\mathbf{r}, t)=-\mathbf{r} \cdot \mathbf{A}(t)$. The translation operator $Q$ is unitary. Then, Eqs. (2.3) is obtained when the function $\Lambda(\mathbf{r}, t)$ is applied to gauge transformation: from the velocity gauge to the length gauge [50].

Note that the electromagnetic force $\mathbf{F}=-\left[\mathbf{E}_{\mathrm{T}}+\mathbf{v} \otimes \mathbf{B}\right]$ should to be invariant under any gauge transformation. We know that $\mathbf{E}_{\mathrm{T}}=\mathbf{E}(t)-\nabla V_{0}(\mathbf{r})$ is the total electric field experienced by the electron. These fields $\mathbf{E}_{\mathrm{T}}$ and $\mathbf{B}(t)$ (induction magnetic field) are gauge invariant as well as the electron trajectory $\mathbf{r}(t)$ and the kinetic momentum 
$\boldsymbol{\pi}=m_{e} \mathbf{v}$ with the electron particle velocity defined by $\mathbf{v}=\frac{d \mathbf{r}}{d t}$.

The canonical momentum $\mathbf{p}$ is not gauge invariant as it is defined by the vector potential $\mathbf{A}(t)$ and the potential $\phi[86]$. In this thesis we do not perform a rigorous demonstration of such a gauge invariance of the physics quantities and we invite the readers to look at, e.g. $[50,86]$.

In contrast to the wavefunction and the Hamiltonian, which are gauge dependent, the TDSE is gauge invariant. This means the observables of the system, in general, are not depending on the gauge choice [50, 88]. Nevertheless, the physical interpretation might change, i.e., there is no potential barrier in velocity gauge where the electron tunnels through.

\subsubsection{Time evolution operator}

The problem of solving the differential equation (2.1) is most often stated as an initial value problem, i.e., at some reference time $t_{0}$, the wavefunction is supposed to be known for the whole space $\Psi\left(\mathbf{r}, t_{0}\right)$. Thus, we can ask how this initial wavefunction evolves in time. To this end, an operator that formally solves the problem is introduced [86]. This operator is called the time evolution operator $U\left(t, t_{0}\right)$ and evolves the wavefunction $\Psi\left(\mathbf{r}, t_{0}\right)$, from the beginning time $t_{0}$ to the posterior time $t$ according to:

$$
\Psi(\mathbf{r}, t)=U\left(t, t_{0}\right) \Psi\left(\mathbf{r}, t_{0}\right) .
$$

In addition, the differential equation which describes the time evolution of the operator $U\left(t, t_{0}\right)$ reads:

$$
i \frac{\partial}{\partial t} U\left(t, t_{0}\right)=H(t) U\left(t, t_{0}\right) .
$$

This is the well known Schrödinger equation for the time-evolution operator. It is equivalent to the TDSE equation but now the evolution of the system is performed by the acting of the unknown evolution operator $U\left(t, t_{0}\right)$ on the wavefunction of the system.

The normalization of an arbitrary wavefunction $\widetilde{\Psi}\left(\mathbf{r}, t_{0}\right)$ can be done:

$$
\Psi\left(\mathbf{r}, t_{0}\right) \equiv \frac{1}{\sqrt{\mathcal{N}}} \widetilde{\Psi}\left(\mathbf{r}, t_{0}\right)
$$

where, $\mathcal{N}=\left\langle\widetilde{\Psi}\left(t_{0}\right) \mid \widetilde{\Psi}\left(t_{0}\right)\right\rangle=\int d \mathbf{r} \widetilde{\Psi}^{*}\left(\mathbf{r}, t_{0}\right) \widetilde{\Psi}\left(\mathbf{r}, t_{0}\right)$ defines the norm of the wavefunction. 


\section{SCHRÖDINGER FORMALISM}

Then, $\Psi(\mathbf{r}, t)$ has the following properties:

$$
\begin{aligned}
\left\langle\Psi\left(t_{0}\right) \mid \Psi\left(t_{0}\right)\right\rangle & =1 \\
\langle\Psi(t) \mid \Psi(t)\rangle & =\left\langle\Psi\left(t_{0}\right)\left|U^{\dagger}\left(t, t_{0}\right) U\left(t, t_{0}\right)\right| \Psi\left(t_{0}\right)\right\rangle, \\
& =1 .
\end{aligned}
$$

Eqs. (2.10) and (2.11) must hold for any choice of $\Psi\left(\mathbf{r}, t_{0}\right)$. According to Sakurai [86], we have the property:

$$
U^{\dagger}\left(t, t_{0}\right) U\left(t, t_{0}\right)=\mathbf{1}
$$

which means that $U\left(t, t_{0}\right)$ is an unitary operator. Another feature we require for the $U$ operator is the composition property:

$$
U\left(t_{2}, t_{0}\right)=U\left(t_{2}, t_{1}\right) U\left(t_{1}, t_{0}\right), \quad\left(t_{2}>t_{1}>t_{0}\right) .
$$

The last relation can be used recursively to decompose the total time evolution into many shorter time steps. For instance, a finite time evolution operator $U\left(t_{0}+\Delta t, t_{0}\right)$ can be constructed from an infinite number of infinitesimal time evolution operators [89]:

$$
U\left(t_{0}+\Delta t, t_{0}\right)=U\left(t_{0}+\Delta t, t_{0}+(N-1) \delta t\right) \ldots U\left(t_{0}+2 \delta t, t_{0}+\delta t\right) U\left(t_{0}+\delta t, t_{0}\right),
$$

where the infinitesimal $\delta t=\frac{\Delta t}{N}$ limit is obtained as the number of subdivisions $N$ approaches infinity. Note that the time ordering of the operators must be remain, otherwise the time evolution will not follow a time sequential. Other property which can be demonstrated by the Taylor expansion of $U\left(t, t_{0}\right)$ around $t_{0}$, as well as taking into account Eq. (2.8), is [86]:

$$
U\left(t_{0}+d t, t_{0}\right)=\mathbf{1}-i H\left(t_{0}\right) \delta t .
$$

The infinitesimal time evolution operator is unitary as required, since the Hamiltonian operator is Hermitian and terms of the order $(\delta t)^{2}$ can be neglected in the limit $\delta t \rightarrow 0$. 


\subsubsection{Time independent Hamiltonians}

In the special case when the Hamiltonian of the system is time independent, $H(t)=H_{0}$, the equation (2.14) for the finite time evolution operator can be written:

$$
U\left(t_{0}+\Delta t, t_{0}\right)=\lim _{N \rightarrow \infty} U\left(t_{0}+\frac{\Delta t}{N}, t_{0}\right)^{N}=\lim _{N \rightarrow \infty}\left(\mathbf{1}-i H_{0} \frac{\Delta t}{N}\right)^{N}=e^{-i H_{0} \Delta t} .
$$

It is easy to see that such a time evolution operator is unitary as it is expected. Note that the evolution of the wavefunction $\Psi\left(\mathbf{r}, t_{0}\right)$ at certain initial time $t_{0}$ will be defined by the application of this operator Eq. (2.17). The latter is fulfilled only in case that the Hamiltonian is time-independent.

\subsubsection{Time dependent Hamiltonians}

When the Hamiltonian is time dependent, it is unfortunately impossible to obtain a simple expression for the time evolution operator like in last section 2.1.4. According to Sakurai [86], if the Hamiltonian of the system commute at different times, the formal generalization of Eq. (2.17) reads:

$$
U\left(t_{1}, t_{0}\right)=e^{-i \int_{t_{0}}^{t_{1}} H(t) d t},
$$

with, $t_{1}=t_{0}+\Delta t$. The above mathematical expression can be easily proved by integrating Eq. (2.1). The application of the operator $U\left(t_{1}, t_{0}\right)$ to the initial wavefunction $\Psi\left(\mathbf{r}, t_{0}\right)$ makes evolve the wavefunction until the time $t_{1}$. Thus, if the integration interval is from the initial time $t_{0}$ to the final time $t_{f}\left(t_{f}>t_{0}\right)$, we can split the time domain $\left[t_{0}, t_{f}\right]$ in $N$ points. Then, applying the property (2.13) the solution for each $t_{n}$ can be directly obtained. Here, we denote $n=0, \ldots, N$.

Furthermore, a special case emerges when the Hamiltonian of the system does not commute at different times. For instance, for a spin-magnetic momentum subjected to a magnetic field where its strength and direction can change in time [86].

In the particular problems addressed throughout this thesis we will restrict ourselves to systems in which the Hamiltonians commute at different times. Then, Eq. (2.17) will perfectly describe the time evolution operator and with it the evolution of the wavefunction. 


\section{STRONG FIELD APPROXIMATION}

\subsubsection{Observables in Quantum Mechanics}

Let us say that an observable $O$ is described by a Hermitian operator acting on a Hilbert space. This canonical observable can be understood as the position, momentum, kinetic energy or potential energy of the system. Then, the expectation value of $O$ is defined by:

$$
\langle O(t)\rangle=\frac{\int d \mathbf{r} \Psi^{*}(\mathbf{r}, t) O \Psi(\mathbf{r}, t)}{\int d \mathbf{r} \Psi^{*}(\mathbf{r}, t) \Psi(\mathbf{r}, t)}
$$

Here, $\Psi(\mathbf{r}, t)$, describes the space-time wavefunction. The integral in this formula is a three-dimensional one and, for instance, $d \mathbf{r}=d x d y d z$ in cartesian coordinates. Furthermore, there are others observables such as the position $\mathrm{S}_{e}(\mathbf{r}, t)=|\Psi(\mathbf{r}, t)|^{2}$ or momentum $\mathrm{S}_{e}(\mathbf{p}, t)=\left|\Psi_{\mathbf{c}}(\mathbf{p}, t)\right|^{2}$ electron density. We will term $\mathrm{S}_{e}(\mathbf{p}, t)$ as the momentum distribution and $P_{c}(\mathbf{p}, t)=\left|\Psi_{c}(\mathbf{p}, t)\right|^{2} d \mathbf{p}$ the momenutm probability to find the electron with a certain momentum $\mathbf{p}$.

The momentum distribution can then be obtained by two different ways. The first one is by projecting on the so-called scattering waves and the second one, on plane waves. In section 2.2.1.8, we will address the definition these electron distribution with more details. In section 2.2.1.8, we will address in detail how these observables are numerically calculated.

\subsubsection{Strong field approximation}

So far we have given the general Physics tools to describe the laser-matter interaction according to the Schrödinger picture. This is the most exact theory to study the interaction between a strong laser and an atomic or molecular system. However, the analytical analysis of the solution for such a TDSE is practically impossible if no approximations are applied to the problem.

In this section, we will address the general description of an approximated model to describe the strong field laser interaction with matter. This approach is the so-called Strong Field Approximation. The SFA has been successfully applied to the study of the photoelectron ionization processes by an ultrashort laser field, amongst other strong field phenomena $[3,6,90]$.

According to Mulser and Bauer [50] if the initial state at the time $t_{0}$ is in a bound state $\left|\Psi_{0}\right\rangle$ of the field-free Hamiltonian $H_{0}$ (let us say this state is the ground state), the complex bound-free transition $a(\mathbf{p}, t)$ to a final continuum state $\left|\Psi_{\mathbf{p}}\right\rangle$ of asymptotic momentum $\mathbf{p}$ reads: 


$$
a(\mathbf{p}, t)=\left\langle\Psi_{\mathbf{p}}\left|U\left(t, t_{0}\right)\right| \Psi_{0}\right\rangle,
$$

where $U\left(t, t_{0}\right)$ is the time evolution operator. Thus, our main task is to determine how this time evolution operator acts on the initial state to bring it to a final state. The time-evolution operator $U\left(t, t_{0}\right)=U^{\dagger}\left(t_{0}, t\right)$ fulfils the Eq. (2.8). The TDSE in the Schrödinger picture reads [86]:

$$
i \frac{\partial}{\partial t}|\Psi(t)\rangle=H(t)|\Psi(t)\rangle, \quad H(t)=\frac{1}{2}[\mathbf{p}+\mathbf{A}(t)]^{2}+V_{0}(\mathbf{r})
$$

Here the Hamiltonian $H(t)$ of the system is given in the velocity gauge. This Hamiltonian can be written in several forms, for example:

$$
H(t)=H_{0}+V_{\mathrm{i}, \mathrm{VG}}(t), \text { or } H(t)=H^{(v)}(t)+V_{0}(\mathbf{r})
$$

In (2.21) the Hamiltonian $H_{0}=\frac{\mathbf{p}^{2}}{2}+V_{0}(\mathbf{r})$ describes the unperturbed atom and the Hamiltonian $H^{(v)}(t)=\frac{\mathbf{p}^{2}}{2}+V_{\mathrm{i}, \mathrm{VG}}(t)$ the electron interacting with an electromagnetic field. This $H^{(v)}$ is the so-called Gordon-Volkov Hamiltonian. The potentials $V_{0}$ and $V_{\mathrm{i}, \mathrm{VG}}$ are defined in section 2.1.1. It is interesting to note that $H_{0}$ does not depend on the gauge choice. However, note, that the momentum $\mathbf{p}$ it is the canonical momentum which depends on gauge choise.

\subsubsection{Volkov states}

As it was pointed out in the last Section, the Gordon-Volkov-Hamiltonian $H^{(v)}(t)$ describes the electron motion under the action of the laser field [91, 92]. The Volkov state $\left|\Psi_{\mathbf{p}}^{(v)}(t)\right\rangle$ satisfies in the Schrödinger picture and velocity gauge:

$$
i \frac{\partial}{\partial t}\left|\Psi_{\mathbf{p}}^{(v)}(t)\right\rangle=H^{(v)}(t)\left|\Psi_{\mathbf{p}}^{(v)}(t)\right\rangle=\frac{1}{2}[\mathbf{p}+\mathbf{A}(t)]^{2}\left|\Psi_{\mathbf{p}}^{(v)}(t)\right\rangle
$$

According to [91, 92] in the dipole approximation the Volkov-Hamiltonian is diagonal in momentum space. Then, the solution of Eq. (2.22) is:

$$
\left|\Psi_{\mathbf{p}}^{(v)}\left(t, t_{0}\right)\right\rangle=\exp \left[-i S_{\mathbf{p}}\left(t, t_{0}\right)\right]|\mathbf{p}\rangle, \quad S_{\mathbf{p}}\left(t, t_{0}\right)=\frac{1}{2} \int_{t_{0}}^{t} d t^{\prime}\left[\mathbf{p}+\mathbf{A}\left(t^{\prime}\right)\right]^{2},
$$




\section{STRONG FIELD APPROXIMATION}

where $|\mathbf{p}\rangle$ are the momentum states $\langle\mathbf{r} \mid \mathbf{p}\rangle=\exp (i \mathbf{p} \cdot \mathbf{r}) /(2 \pi)^{3 / 2}$. In the last equation we have dropped out the lower index VG in the state $\left|\Psi_{\mathbf{p}}^{(v)}\left(t, t_{0}\right)\right\rangle$ to simplify our notation. On the other hand, we name the phase $S_{\mathbf{p}}\left(t, t_{0}\right)$ as the Volkov phase. As it is mentioned above, the transition from the velocity gauge to the length gauge corresponds to a translation on the momentum, then it is easy to show that the Volkov state $\left|\Psi_{\mathbf{p}, \mathrm{LG}}^{(v)}\left(t, t_{0}\right)\right\rangle$ in the length gauge is:

$$
\left|\Psi_{\mathbf{p}, \mathrm{LG}}^{(v)}\left(t, t_{0}\right)\right\rangle=\exp \left[-i S_{\mathbf{p}}\left(t, t_{0}\right)\right]|\mathbf{p}+\mathbf{A}(t)\rangle
$$

For the Volkov states we can call $S_{\mathbf{p}}\left(t, t_{0}\right)$ as the action of the system defined in Eq. (2.23).

\subsubsection{Complex transition matrix element}

Now we continue with the derivation of the SFA transition matrix element $a(\mathbf{p}, t)$ (Eq. 2.19). We stress out that our main task is to find out how the time-evolution operator $U\left(t, t_{0}\right)$ acts on a given initial state. Under the splitting of the Hamiltonian $H(t)=H_{0}+V_{\mathrm{i}}(t)$ and by integrating Eq. (2.8), we obtain the integral form for this partial differential equation $[86,93]$ :

$$
\begin{aligned}
U\left(t, t_{0}\right) & =U_{0}\left(t, t_{0}\right)-i \int_{t_{0}}^{t} d t^{\prime} U\left(t, t^{\prime}\right) V_{\mathrm{i}}\left(t^{\prime}\right) U_{0}\left(t^{\prime}, t_{0}\right), \\
& =U_{0}\left(t, t_{0}\right)-i \int_{t_{0}}^{t} d t^{\prime} U_{0}\left(t, t^{\prime}\right) V_{\mathrm{i}}\left(t^{\prime}\right) U\left(t^{\prime}, t_{0}\right) .
\end{aligned}
$$

Here $U_{0}\left(t, t_{0}\right)$ denotes the evolution operator corresponding to the field-free Hamiltonian $H_{0}$. By inserting Eq. (2.25) in the transition matrix element Eq. (2.19) it is easy to find that:

$$
a(\mathbf{p}, t)=-i \int_{t_{0}}^{t} d t^{\prime}\left\langle\Psi_{\mathbf{p}}\left|U\left(t, t^{\prime}\right) V_{\mathrm{i}}\left(t^{\prime}\right)\right| \Psi_{0}\left(t^{\prime}\right)\right\rangle
$$

where, $\left\langle\Psi_{\mathbf{p}}\left|U_{0}\left(t, t_{0}\right)\right| \Psi_{0}\right\rangle=\left\langle\Psi_{\mathbf{p}} \mid \Psi_{0}(t)\right\rangle=0$. This is the case because $\left|\Psi_{p}\right\rangle$ is an eigenvector of the Hamiltonian $H_{0}$ as well as $\left|\Psi_{0}(t)\right\rangle=U\left(t, t_{0}\right)\left|\Psi_{0}\right\rangle=e^{-i E_{0}\left(t-t_{0}\right)}\left|\Psi_{0}\right\rangle$, where $E_{0}$ is the binding energy of the bound state, i.e., the ground state. The state $\left|\Psi_{0}\left(t^{\prime}\right)\right\rangle$ is obtained by $\left|\Psi_{0}\left(t^{\prime}\right)\right\rangle=U\left(t^{\prime}, t_{0}\right)\left|\Psi_{0}\right\rangle$.

Furthermore, under the choice of the Hamiltonian, $H(t)=H^{(v)}(t)+V_{0}(\mathbf{r})$, the evolution 
operator $U\left(t, t_{0}\right)$ should satisfy the integral equation [50, 93]:

$$
\begin{aligned}
U\left(t, t_{0}\right) & =U^{(v)}\left(t, t_{0}\right)-i \int_{t_{0}}^{t} d t^{\prime} U^{(v)}\left(t, t^{\prime}\right) V_{0}(\mathbf{r}) U\left(t^{\prime}, t_{0}\right), \\
& =U^{(v)}\left(t, t_{0}\right)-i \int_{t_{0}}^{t} d t^{\prime} U\left(t, t^{\prime}\right) V_{0}(\mathbf{r}) U^{(v)}\left(t^{\prime}, t_{0}\right),
\end{aligned}
$$

where $U^{(v)}\left(t, t_{0}\right)$ denotes the time-evolution operator corresponding to the TDSE in Eq. (2.22) and $V_{0}(\mathbf{r})$ is the electrostatic potential energy of the system without laser field. Then, by inserting Eq. (2.27) in (2.26) the transition amplitude yields:

$$
\begin{aligned}
a(\mathbf{p}, t)= & -i\left[\int_{t_{0}}^{t} d t^{\prime}\left\langle\Psi_{\mathbf{p}}\left|U^{(v)}\left(t, t^{\prime}\right) V_{\mathrm{i}}\left(t^{\prime}\right)\right| \Psi_{0}\left(t^{\prime}\right)\right\rangle\right. \\
& \left.-i \int_{t_{0}}^{t} d t^{\prime} \int_{t^{\prime}}^{t} d t^{\prime \prime}\left\langle\Psi_{\mathbf{p}}\left|U^{(v)}\left(t, t^{\prime \prime}\right) V_{0}(\mathbf{r}) U\left(t^{\prime \prime}, t^{\prime}\right) V_{\mathrm{i}}\left(t^{\prime}\right)\right| \Psi_{0}\left(t^{\prime}\right)\right\rangle\right] .
\end{aligned}
$$

This equation is equivalent to the whole solution of the TDSE for the ionization transition amplitude mediated by an strong ultrashort IR laser field. This is the case, because the unknown time-evolution operator $U\left(t^{\prime \prime}, t^{\prime}\right)$ remains in the second line. The first integral term in Eq. (2.28) corresponds to the electrons that can be ionized directly by the strong field $[78,79,80]$. This is so, because the evolution of the system comes from an state $\left|\Psi_{0}\left(t^{\prime}\right)\right\rangle$ to another state which is influence by the interacting potential energy $V_{\mathrm{i}}\left(\mathbf{r}, t^{\prime}\right)$. Then, the result is evolved by the Volkov-evolution operator $U^{(v)}\left(t, t^{\prime}\right)$ to a final state $\left|\Psi_{\mathbf{p}}\right\rangle$ of asymptotic momentum p. Throughout this "evolution path" the electron does not explicitly interact with the ion core after been ionized. Nevertheless, the second double integral term (second line in Eq. (2.28)) means that once the electron is ionized there is a probability that the electron interacts with the ion core. When this interaction occurs with the potential without laser, the second term asks to the system if the final state is promoted to a continuum state $\left|\Psi_{\mathbf{p}}\right\rangle$. Thus, the second term is related to a re-scattering process with the core potential [93].

In addition, the main problems that we will address throughout this thesis does not take into account the re-scattering process. We can neglect the second line terms in Eq. (2.28).

The main assumption of the SFA is that the final state $U^{(v)}\left(t, t^{\prime}\right)\left|\Psi_{\mathbf{p}}\right\rangle$ corresponds to a Volkov state when the binding Coulomb potential is neglected as well. This mean that the final state can easily be described by Eq. (2.23). Thus, the transition complex 


\section{STRONG FIELD APPROXIMATION}

amplitude reads:

$$
a(\mathbf{p}, t)=-i \int_{t_{0}}^{t} d t^{\prime}\left\langle\Psi_{\mathbf{p}}^{(v)}\left(t^{\prime}, t\right)\left|V_{\mathrm{i}}\left(t^{\prime}\right)\right| \Psi_{0}\left(t^{\prime}\right)\right\rangle
$$

with,

$$
\left\langle\Psi_{\mathbf{p}}^{(v)}\left(t^{\prime}, t\right)\right|=\left\langle\Psi_{\mathbf{p}}^{(v)}\right| U^{(v)}\left(t, t^{\prime}\right)=\langle\mathbf{p}| e^{i S\left(t^{\prime}, t\right)} .
$$

In general the Volkov states $\left|\Psi_{\mathbf{p}}^{(v)}\left(t, t^{\prime}\right)\right\rangle$ and the interacting potential $V_{i}\left(t^{\prime}\right)$ will depend on the gauge chosen to describe the interaction process (see Eqs. (2.3)-(2.4)), namely, the velocity or the length gauge. The election of gauge will be addressed in the corresponding applications throughout this thesis.

Let us assume that a XUV attosecond pulse with electric field, $\mathbf{E}_{\mathrm{X}}(t-\tau)$, interacts with the ground state of an atomic system under the SAE approach. If the photoionization is carried out in the presence of a weak IR laser pulse, e.g. $10^{10}-10^{12} \mathrm{~W} / \mathrm{cm}^{2}$ of peak intensity, with vector potential $\mathbf{A}_{\mathrm{L}}(t)$, the complex transition amplitude, $a(\mathbf{p}, t)$, in the length gauge according to SFA is:

$$
a(\mathbf{p}, t)=-i \int_{t_{0}}^{t} d t^{\prime} e^{i S_{\mathbf{p}}\left(t^{\prime}, t\right)}\left\langle\mathbf{p}+\mathbf{A}_{\mathrm{L}}\left(t^{\prime}\right)\left|\mathbf{r} \cdot \mathbf{E}_{\mathbf{X}}\left(t^{\prime}-\tau\right)\right| \Psi_{0}\left(t^{\prime}\right)\right\rangle,
$$

where, $\tau$, will denote the time delay between the maximum envelope of the IR laser and the XUV attosecond pulse. It is worth to mention that the above equation is suitable to describe the ionization under the condition that the IR laser field does not ionize the system or at least the ionization driven by the IR is negligible compared to the XUV one. In addition we have taken advantage of the Volkov states derived in Section 2.1.6.1 to reach Eq (2.25).

Eq. (2.29) is even more simplified by defining the bound-free dipole transition matrix element $\mathbf{d}=-\left\langle\mathbf{p}+\mathbf{A}_{\mathrm{L}}\left(t^{\prime}\right)|\mathbf{r}| \Psi_{0}\right\rangle$ and by addition by considering that $\left|\Psi_{0}\left(t^{\prime}\right)\right\rangle=$ $e^{i I_{p}\left(t^{\prime}-t_{0}\right)}\left|\Psi_{0}\right\rangle[78,79,80,94]$ :

$$
a(\mathbf{p}, t)=i \int_{t_{0}}^{t} d t^{\prime} e^{i\left[I_{p}\left(t^{\prime}-t_{0}\right)-\frac{1}{2} \int_{t^{\prime}}^{t}\left(\mathbf{p}+\mathbf{A}_{\mathrm{L}}\left(t^{\prime \prime}\right)\right)^{2} d t^{\prime \prime}\right]} \mathbf{E}_{\mathrm{X}}\left(t^{\prime}-\tau\right) \cdot \mathbf{d}\left(\mathbf{p}+\mathbf{A}_{\mathrm{L}}\left(t^{\prime}\right)\right),
$$

where $I_{p}$ is the ionization potential of the atomic system. For instance, the length gauge is utilized to model the ionization from an atomic system mediated by two colors pulses scheme. One color is a XUV attosecond pulse and the other one is an moderated ultrashort IR laser field. Then, when the probability amplitude is scanned on the 
time delay between the two colors, the so-called streaking trace is measured [64]. This picture describes quite satisfactory the experimental results for the attosecond streak camera [3].

So far we have derived the SFA equation for the ionization of an atom or molecule carried out by a XUV in the presence of a moderated IR laser field. This derivation will be later employed in the Chapters 4 and 5 to model the attosecond streaking technique and the QSPIDER implementation as well.

\section{$2.2 \quad$ Numerical methods}

In this Section we detail the numerical method utilized to integrate numerically the TDSE and the SFA model.

During the recent years we have developed general tools to solve numerically the TDSE in the $1 \mathrm{D}, 2 \mathrm{D}$ and $3 \mathrm{D}$ cartesian coordinates. We have also implemented the numerical integration of the TDSE in cylindrical and spherical coordinates for problems which take into account that the laser is linearly polarized. These three integration schemes are developed under the SAE and dipole approximation.

In addition, numerical routines to compute observables such as the position electronic density as a function of time, the transition complex amplitude to a continuum state of a certain asymptotic momentum $\mathbf{p}$, the position and momentum expectation values, the ionization yield and the asymmetry amplitude for electrons emitted on the left and right, are implemented.

\subsubsection{Schrödinger equation in cartesian coordinates}

As it is established in Section 2.1.4 when the Hamiltonian commutes at different times and the initial wavefunction is known at $t_{0}$, we can solve the TDSE by Eq. (2.17) to find the evolution of the wavefunction till $t_{0}+\Delta t$. If the time step $\Delta t$ is "small" compared to the time scale of the system, i.e., shorter than an optical cycle of the laser pulses, the integral exponential term of the operator $U\left(t_{0}+\Delta t, t_{0}\right)$ in Eq. (2.17) can be approximated to:

$$
U\left(t_{0}+\Delta t, t_{0}\right)=e^{-i \int_{t_{0}}^{t_{0}+\Delta t} H(t) d t} \approx e^{-i H\left(t_{0}+\frac{\Delta t}{2}\right) \Delta t},
$$

with this, the wavefunction $\Psi\left(\mathbf{r}, t_{0}+\Delta t\right)$ is found by: 


$$
\Psi\left(\mathbf{r}, t_{0}+\Delta t\right) \approx e^{-i H\left(t_{0}+\frac{\Delta t}{2}\right) \Delta t} \Psi\left(\mathbf{r}, t_{0}\right) .
$$

Suppose that the atomic or molecular system interacts with a laser pulse on the time interval $\left[t_{0}, t\right]$. This interval is then divided in $N_{t}$ point according to $t_{n}=t_{0}+n \Delta t$ with $n=0, \ldots, N_{t}-1$. To find the evolution of the wavefunction until the final time $t=t_{0}+\Delta t\left(N_{t}-1\right)$, we apply sequentially the evolution operator Eq. (2.32) in the following way:

$$
\Psi(\mathbf{r}, t)=e^{-i H\left(t_{N_{t}-1}^{\prime}\right) \Delta t} \times \ldots \times e^{-i H\left(t_{1}^{\prime}\right) \Delta t} e^{-i H\left(t_{0}^{\prime}\right) \Delta t} \Psi\left(\mathbf{r}, t_{0}\right) .
$$

Note, that the Hamiltonian $H(t)$ is evaluated at the time middle points $t_{n}^{\prime}=t_{n}+\frac{\Delta t}{2}$. Our main task is to implement numerically the last method, Eq. (2.34), from the $t_{0}$ to $t$ and to compute the wavefunction $\Psi\left(\mathbf{r}, t_{n}\right)$. The latter is needed to calculate observables at each time $t_{n}$. It is important to point out that the application order of the evolution operator in Eq. (2.34) has to be kept.

Several and different numerical methods to solve the TDSE by employing Eq. (2.1) or (2.34) have been developed. For example, some of those methods are: the secondorder differential [95], the Crank-Nicolson [96, 97], spectral spilt-operator methods [98], as well as, a mix of their [99]. Furthermore, other methods to solve the timeindependent Schrödinger equation, e.g. Chebyshev [95, 100], the Lanczos [101], and the time-imaginary propagation method [102] were employed.

In this thesis we will restrict ourselves to use the spectral split-operator and the Crank-Nicolson methods to numerically integrate the TDSE in different coordinate systems, i.e. cartesian, cylindrical and spherical coordinates. The spectral split-operator and Crank-Nicolson methods to solve partial differential equations are stable under any choice of the time grid $\Delta t$ and position grid steps $\Delta x, \Delta y$, and $\Delta z$. This is so, while the relation $\Delta t \leq(\Delta x)^{2}$ will be fulfilled $[97,103]$. It is also important to point out that these two methods conserved the norm of the wavefunction. Thus, these time-evolution schemes are unitary in the sense that the wavefunction does not change its norm at different times.

\subsubsection{Split-operator spectral method}

Feit et al. in reference [98] has introduced the split-operator spectral method to solve numerically the TDSE in 2D. This method consists in to split the time evolution opera- 
tor of Eq. (2.33) in three parts, as it is indicated in Eq. (2.35). Mathematically, one can define the Hamiltonian $H(t)=T+V(t)$ as the sum of the kinetic energy $T=-\frac{1}{2 \mu} \nabla^{2}$ and an effective potential energy operators $V(t)=V_{0}+V_{\mathrm{i}}(t)$. According to [98] we re-write Eq. (2.33) as:

$$
\Psi\left(\mathbf{r}, t_{1}\right)=\underbrace{e^{-i T \Delta t / 2}}_{(i i i)} \overbrace{e^{-i V\left(t_{0}^{\prime}\right) \Delta t}}^{(i i)} \underbrace{e^{-i T \Delta t / 2}}_{(i)} \Psi\left(\mathbf{r}, t_{0}\right)+\operatorname{Ord}\left[(\Delta t)^{3}\right],
$$

with,

$$
\begin{aligned}
& t_{1}=t_{0}+\Delta t, \\
& t_{0}^{\prime}=t_{0}+\frac{\Delta t}{2} .
\end{aligned}
$$

The separation of such an operator $e^{-i[T+V(t)] \Delta t}$ can be written in other way $e^{-i[T+V(t)] \Delta t}=$ $e^{-i V(t) \Delta t / 2} e^{-i T \Delta t} e^{-i V(t) \Delta t / 2}+O\left(\Delta t^{3}\right)[104,105]$. As the kinetic operator $T$ is a second derivative in the position space, its application can be easily evaluated on the momentum-spectral domain. By doing a forward Fourier Transform of $\Psi\left(\mathbf{r}, t_{0}\right)$, the momentum representation of the wavefunction $\Psi\left(\mathbf{p}, t_{0}\right)=(2 \pi)^{-\eta / 2} \int \Psi\left(\mathbf{r}, t_{0}\right) e^{-i \mathbf{p} \cdot \mathbf{r}} d \mathbf{r}$ is computed. Here we define, $\eta$, as a parameter which points out the dimension of the problem. The parameter $\eta$ takes the value of, $\eta=1,2,3$, if the problem is studied in $1 \mathrm{D}, 2 \mathrm{D}$, and $3 \mathrm{D}$, respectively. The application of the first $(i)$ exponential factor in Eq. (2.35) will follow:

$$
\Phi(\mathbf{p})=e^{-i \frac{\mathbf{p}^{2}}{2} \frac{\Delta t}{2}} \Psi\left(\mathbf{p}, t_{0}\right)
$$

Then, by employing an inverse Fourier transform on $\Phi(\mathbf{p})$, the spatial wavefunction $\Phi(\mathbf{r})=(2 \pi)^{-d / 2} \int \Phi(\mathbf{p}) e^{i \mathbf{p} \cdot \mathbf{r}} d \mathbf{p}$ is recovered. Thus, the second exponential term $(i i)$ in Eq. (2.35) can be applied to $\Phi(\mathbf{r})$. This allows us to introduce the evolution given by the effective potential $V(t)$ :

$$
\vartheta(\mathbf{r})=e^{-i V\left(t_{0}^{\prime}\right) \Delta t} \Phi(\mathbf{r})
$$

We do once again a forward FT of $\vartheta(\mathbf{r})$ in order to apply the last exponential term (iii) in Eq. (2.35). The momentum-spectral space of this function is denoted by $\vartheta(\mathbf{p})$. 
Then, we follow the same step given by Eq. (2.36) to obtain the function:

$$
\Psi\left(\mathbf{p}, t_{1}\right)=e^{-i \frac{\mathbf{p}^{2}}{2} \frac{\Delta t}{2}} \vartheta(\mathbf{p}) .
$$

Finally, an inverse FT is applied to $\Psi\left(\mathbf{p}, t_{1}\right)$ in order to find the evolution of the wavefunction $\Psi(\mathbf{r})$ from the time $t_{0}$ to $t_{1}=t_{0}+\Delta t$. Then, by iterating on a "time loop" the Eq. (2.34) the final wavefunction $\Psi(\mathbf{r}, t)$ can be numerically computed.

It is important to note that Eq. (2.35) is only suitable in the case that the interaction potential $V_{\mathrm{i}}(t)$ is written in the length gauge. For the velocity gauge, it is important to rewrite the Hamiltonian $H=\frac{1}{2}[\mathbf{p}+\mathbf{A}(t)]^{2}+V_{0}(\mathbf{r})$. When the velocity gauge is employed to solve the TDSE, we refer to the effective potential in Eq. (2.35) as $V(t)=$ $V_{0}(\mathbf{r})$ and the kinetic energy operator $T=\frac{1}{2}[\mathbf{p}+\mathbf{A}(\mathbf{t})]^{2}$. Under these two conditions, we can use the set of Eqs. (2.36)-(2.38) to obtain the wavefunction evolution from the time $t_{0}$ to $t_{0}+\Delta t$. In the next Chapter 3 , we will address in detail how this split operator algorithm is implemented in cartesian, cylindrical and spherical coordinates.

\subsubsection{Position and momentum space: the grids}

We define the position and momentum space in cartesian coordinates according to:

$$
\mathbf{r} \equiv(x, y, z), \text { and } \mathbf{p} \equiv\left(p_{x}, p_{y}, p_{z}\right)
$$

Here, we denote $G_{\mathbf{r}}$ as the position "grid" in cartesian coordinates and $G_{\mathbf{p}}$ as the momentum "grid". Then, if the grid steps for $\mathrm{G}_{\mathbf{r}}$ on each directions $x, y$ and $z$ are denoted by $\Delta x, \Delta y$ and $\Delta z$, respectively, and the corresponding number of points are $N_{x}, N_{y}$ and $N_{z}$, the axes of the numerical $\mathrm{G}_{\mathbf{r}}$ read:

$$
\begin{aligned}
x_{\underline{i}}=x_{\min }+\underline{i} \Delta x ; & \underline{i}=0, \ldots, N_{x}-1, \\
y_{j}=y_{\min }+\underline{j} \Delta y ; & \underline{j}=0, \ldots, N_{y}-1, \\
z_{\underline{k}}=z_{\min }+\underline{k} \Delta z ; & \underline{k}=0, \ldots, N_{z}-1,
\end{aligned}
$$

respectively, where $x_{\min }=-\frac{N_{x}}{2} \Delta x$, and with equivalent definitions for $y_{\min }$ and $z_{\min }$. The elements $x_{\underline{i}}, y_{\underline{j}}$ and $z_{\underline{k}}$ are computationally implemented in the vector arrays $\mathbf{x}=\left\{x_{0}, x_{1}, \ldots, x_{N_{x}-1}\right\} ; \mathbf{y}=\left\{y_{0}, y_{1}, \ldots, y_{N_{y}-1}\right\} ;$ and $\mathbf{z}=\left\{z_{0}, z_{1}, \ldots, z_{N_{z}-1}\right\}$. This means that we can represent $\mathbf{x}=\sum_{\underline{i}=0}^{N_{x}-1} x_{\underline{i}} \mathbf{e}_{\underline{i}}$, where $\left\{\mathbf{e}_{0}, \mathbf{e}_{1}, \ldots, \mathbf{e}_{N_{x}-1}\right\}$ is a unit vector of the vectorial space and $x_{\underline{i}}$ the corresponding component [89].

The momentum grid $\mathbf{G}_{\mathbf{p}}$ is defined by taking into account the Nyquist critical frequency 
[103]. Its relation with the $\mathrm{G}_{\mathbf{r}}$ parameters are as follows: on the one hand the maximum momentum grid values on each direction, $p_{\mathrm{xmax}}=\frac{\pi}{\Delta x}, p_{\mathrm{ymax}}=\frac{\pi}{\Delta y}$, and $p_{\mathrm{zmax}}=\frac{\pi}{\Delta z}$, and on the other hand, the $\mathrm{G}_{\mathbf{p}}$ sampling $\Delta p_{x}=\frac{2 \pi}{N_{x} \Delta x}, \Delta p_{y}=\frac{2 \pi}{N_{y} \Delta y}$, and $\Delta p_{z}=\frac{2 \pi}{N_{z} \Delta z}$. Thus, the $G_{\mathbf{p}}$ can be written as:

$$
\begin{array}{ll}
p_{x, \underline{i}}=-p_{\operatorname{xmax}}+\underline{i} \Delta p_{x} ; & \underline{i}=0, \ldots, N_{x}-1, \\
p_{y, \underline{j}}=-p_{\operatorname{ymax}}+\underline{j} \Delta p_{y} ; & \underline{j}=0, \ldots, N_{y}-1, \\
p_{z, \underline{k}}=-p_{\mathrm{zmax}}+\underline{k} \Delta p_{z} ; & \underline{k}=0, \ldots, N_{z}-1 .
\end{array}
$$

The computational implementation for such a numerical position and momentum space grids will be addressed in the Chapter 3 .

\subsubsection{Hamiltonian of the Systems}

The main interest to perform a simulation model of the interaction between a laser pulse and an atomic or molecular system, is to access to the time-evolution of the wavefunction $\Psi(\mathbf{r}, t)$. As it is known, full information about the time-evolution of the observables and structure of the electron system is encoded in such a quantity. This is the main reason why so much effort has been put on the numerical and analytical solution of the TISE and TDSE. Thus, our task is devoted to find the initial wavefunction $\Psi_{0}(\mathbf{r})$ of the system before the interaction with the laser, $\Psi(\mathbf{r}, t)$, i.e. its evolution during the interaction and, finally, $\Psi\left(\mathbf{r}, t_{f}\right)$, i.e the wavefunction after the interaction with the laser is turned off.

\section{Wavefunction}

The wavefunction $\Psi(\mathbf{r}) \equiv \boldsymbol{\Psi}$ can be numerically represented in a third-order array given by:

$$
\boldsymbol{\Psi}=\sum_{\underline{k}, \underline{j}, \underline{i}} \Psi_{\underline{k} \underline{j} \underline{j}} \mathbf{e}_{\underline{k} \underline{j} \underline{i}},
$$

where, $\sum_{\underline{k}, \underline{j}, \underline{i}}=\sum_{\underline{k}=0}^{N_{z}-1} \sum_{\underline{j}=0}^{N_{y}-1} \sum_{\underline{i}=0}^{N_{x}-1}$, denotes the sum over the three indexes. Here $\mathbf{e}_{\underline{k} \underline{j} \underline{j}} \equiv \mathbf{e}_{\underline{k}} \otimes \mathbf{e}_{\underline{j}} \otimes \mathbf{e}_{\underline{i}}$ denotes the basis where the wavefunction has been discretized on $\mathrm{G}_{\mathbf{r}}$. This basis is created by the tensor product or external product of the $\mathbf{e}_{x}, \mathbf{e}_{y}$ and $\mathbf{e}_{z}$. This means that the wavefunction $\Psi(\mathbf{r})$ "live" in a array of third order. Note that in the $2 \mathrm{D}$ case the components of $\Psi(\mathbf{r})$ are represented in a matrix or second-order array with $\Psi_{\underline{j}, \underline{i}}=\Psi\left(y_{\underline{j}}, x_{\underline{i}}\right)$. In the $1 \mathrm{D}$ model the wavefunction $\Psi(\mathbf{r})$ will be represented in a 
vector array with elements $\Psi_{\underline{i}}=\Psi\left(x_{\underline{i}}\right)$ or $\Psi_{\underline{k}}=\Psi\left(z_{\underline{k}}\right)$.

\section{Hamiltonian}

The general Hamiltonian $H(t)$ of the system can be split into two parts: the timeindependent $H_{0}=\frac{\mathbf{p}^{2}}{2}+V_{0}(\mathbf{r})$, and the time-dependent $V_{\mathrm{i}}(t)$ one as it is pointed out in Eq. (2.20).

The potential energy functions $V_{0}(\mathbf{r})$ utilized in this thesis to model the atomic interaction (electron-nucleus) under the SAE approach are: the Hydrogen-like softcore Coulomb potential (this is a kind of long-range potential) and the soft-core Yukawa potential (this a kind of short-range potential):

$$
\begin{aligned}
& V_{0}(\mathbf{r})=-\frac{Z}{\sqrt{a+x^{2}+y^{2}+z^{2}}} \quad \text { Hydrogen-like soft-core Coulomb potential } \\
& V_{0}(\mathbf{r})=-\frac{Z \exp \left[-\frac{\sqrt{a+x^{2}+y^{2}+z^{2}}}{a_{s}}\right]}{\sqrt{a+x^{2}+y^{2}+z^{2}}} \quad \text { Soft-core Yukawa potential }
\end{aligned}
$$

where, $Z$, is an effective charge parameter for the atomic nucleus, $a$, the soft-core parameter and, $a_{s}$, the screening length in case of a Yukawa potential.

In case of molecular systems we suppose molecules with the two nuclei centers fixed, e.g. $\mathrm{CO}$ or $\mathrm{H}_{2}^{+}$. The potential well for these cases is defined by:

$$
V_{0}(\mathbf{r})=-\frac{Z_{1}}{\sqrt{a_{1}+x^{2}+y^{2}+\left(z+\frac{R_{0}}{2}\right)^{2}}}-\frac{Z_{2}}{\sqrt{a_{2}+x^{2}+y^{2}+\left(z-\frac{R_{0}}{2}\right)^{2}}},
$$

where, $a_{1}$, and, $a_{2}$, are the soft-core parameters to the two cores which are located at $z=-\frac{R_{0}}{2}$ and $z=\frac{R_{0}}{2}$, respectively. Here, $R_{0}$, is the internuclear distance between the two nuclei. The parameters $Z_{1}$ and $Z_{2}$ denote the nuclei charges for each atom.

\subsubsection{Discrete and continuum spectrum}

To compute the initial wavefunction, $\Psi_{0}(\mathbf{r})$, of the system we will take into account the Hamiltonian $H_{0}$. Thus, our problem is reduced to compute the TISE for the system under study, namely:

$$
-\frac{1}{2} \nabla^{2} \Psi_{n}(\mathbf{r})+V_{0}(\mathbf{r}) \Psi_{n}(\mathbf{r})=E_{n} \Psi_{n}(\mathbf{r}) .
$$


$E_{n}$ denotes the eigenenergy value of the corresponding wavefunction or eigenstate $\Psi_{n}$. It is important to mention that the energy spectrum is divided in: the discrete or bound spectrum and the continuum spectrum $[86,106]$. This classification arises from that fact the energy $E_{n}$ is negative and discrete for the eigenstates $\Psi_{n}$. The set of energy values $E_{n}<0$ corresponds to the wavefunctions that are bounded by the potential well. On the other hand, the continuum spectrum is defined by the wavefunctions, $\Psi_{k}(\mathbf{r})$, with energies $E_{k}>0$. Here, we have changed the notation of $E_{n}$ to $E_{k}$ to point out that $n$ is a discrete variable and $k$ is a continuum one ${ }^{1}$.

In order to find out the bound states $\Psi_{n}(\mathbf{r})$ for a given potential well $V_{0}(\mathbf{r})$, we can diagonalize $H_{0}$ by employing two methods: the Lanczos and the imaginary-time propagation methods. The Lanczos method is only implemented in 1D.

The continuum spectrum is calculated by the Numerov Method [107] only in 1D, too. The continuum wavefunction $\Psi_{k}(\mathbf{r})$ for $2 \mathrm{D}$ and $3 \mathrm{D}$ in cartesian coordinates is a complicated computational task by the diagonalization method. The determination of the normalization coefficients can be a hard task to do as well. However, this computation can be made as it is explained in reference [107]. In this thesis we have implemented only the scattering wave in $1 \mathrm{D}$.

\section{Imaginary time propagation method}

A simple numerical method to compute the bound states is the so-called inverse imaginary time propagation [102]. The ground state of a general potential energy well $V_{0}(\mathbf{r})$ is calculated by solving Eq. (2.17) considering the time-evolution operator given by Eq. (2.7). Thus, the inverse imaginary time evolution is obtained by using $t \rightarrow-i \tau^{\prime}$ :

$$
\Psi_{0}\left(\mathbf{r}, \tau^{\prime}+\Delta \tau^{\prime}\right)=e^{-H_{0} \Delta \tau^{\prime}} \Psi_{g}(\mathbf{r})
$$

$H_{0}$, is the field-free Hamiltonian of the system. The propagation scheme is different and the symbol $\tau^{\prime}$ is now a real parameter that can not be confused with a physical time. It is important to note that the propagator $U\left(\tau^{\prime}+\Delta \tau^{\prime}\right)=e^{-H_{0} \Delta \tau^{\prime}}$, is now non-unitary. This mean that the norm is no time-conserved and the propagated wavefunction does not represent the physical state of a particle [89].

The wavefunction $\Psi_{g}(\mathbf{r})$ denotes a guess square integrable function, i.e., a gaussian function. Then, by numerically evolving Eq. 2.47 the ground state of the general

\footnotetext{
${ }^{1}$ The momentum, $k$, will denotes the module wave vector of the wave vector $\mathbf{k}$, and it is the so-called asymptotic momentum of the particle as well.
} 
Hamiltonian $H_{0}$ can be obtained. This is the case because formally we can expand $\Psi_{g}(\mathbf{r})$ as a linear combination of the eigenstates $\left\{\Phi_{n}(\mathbf{r})\right\}$ of $H_{0}$. Thus, the guess function becomes:

$$
\begin{aligned}
\Psi_{0}\left(\mathbf{r}, \tau^{\prime}+\Delta \tau^{\prime}\right) & =e^{-H_{0} \Delta \tau^{\prime}} \Psi_{g}(\mathbf{r}) \\
& =\sum_{n} e^{-E_{n} \Delta \tau^{\prime}} c_{n} \Phi_{n}(\mathbf{r})
\end{aligned}
$$

This equation suggests that the different components of the spectral decomposition of $\Psi_{g}\left(\mathbf{r}, \tau^{\prime}\right)$ decay or explode exponentially as $\tau^{\prime} \rightarrow \infty$ increase. If the spectrum contains negative and positive eigenenergies, the eigenstate corresponding to the component of less energy (most negative energy) will increase quickly compared to the higher ones. Eq. (2.48) tell us that the ground state $\Psi_{0}(\mathbf{r})$ of the Hamiltonian $H_{0}$ can be obtained when we imaginary evolve or propagate the guess function $\Psi_{g}(\mathbf{r})$.

The ground state energy, $E_{0}$, can be obtained by re-normalizing the wavefunction $\Psi_{0}\left(\mathbf{r}, \tau^{\prime}\right)$ using Eq. (2.9) each iteration time $\tau^{\prime}=\tau_{0}^{\prime}, \tau_{0}^{\prime}+\Delta \tau^{\prime}, \tau_{0}^{\prime}+2 \Delta \tau^{\prime}, \ldots$ and computing the expectation value of $H_{0}$. Thus, the energy $E_{\tau^{\prime}}$ should converge to the ground state energy when $\tau^{\prime} \rightarrow \infty$. This means that a criterion to ensure can be defined. This is by computing the difference $\left|E_{\tau_{0}^{\prime}+\Delta \tau}-E_{\tau_{0}^{\prime}}\right| \rightarrow 0$. We can reach the ground state wavefunction, namely to compute the ground state energy until convergence [89].

The imaginary time propagation method is successfully applied to compute the ground state of any Hamiltonian $H_{0}$. This method also can be employed to compute the first excited states as well. However, the accuracy of the solution for higher energy states can be smaller than the one for the ground state [89].

Furthermore, it is important to mention that this method can be easily applied in $1 \mathrm{D}, 2 \mathrm{D}$ and $3 \mathrm{D}$, and in any coordinate system, i.e., cartesian, cylindrical and spherical coordinates.

\section{Finite difference method or Lanczos matrix in 1D}

Another method to compute the set of bound states or continuum states is the diagonalization matrix method or the Finite difference method. The full time-independent Schrödinger equation in 1D for the wavefunction $\Psi(z)$ in the grid discretization can be written:

$$
\sum_{\underline{k}=0}^{\mathrm{N}_{z}-1}\left[-\frac{1}{2 \mu} \frac{\mathbf{e}_{\underline{k}+1}-2 \mathbf{e}_{\underline{k}}+\mathbf{e}_{\underline{k}-1}}{h^{2}}+V_{0, \underline{k}} \mathbf{e}_{\underline{k}}\right] \Psi_{\underline{k}}=E \sum_{\underline{k}=0}^{\mathrm{N}_{z}-1} \Psi_{\underline{k}} \mathbf{e}_{\underline{k}},
$$


with $h \equiv \Delta z$. If we multiply (inner product) by $\mathbf{e}_{\underline{j}}$ we obtain:

$$
\begin{aligned}
\sum_{\underline{k}=0}^{\mathrm{N}_{z}-1}\left(-\frac{1}{2 \mu}\left[D_{2}\right]_{\underline{k}, \underline{j}}+\left[V_{0}\right]_{\underline{k}, \underline{j}}\right) \Psi_{\underline{k}} & =E \sum_{\underline{k}=0}^{\mathrm{N}_{z}-1}[I]_{\underline{k}, \underline{j}} \Psi_{\underline{k}} \\
{\left[-\frac{1}{2} \mathbf{D}_{2}+\left(\mathbf{V}_{0}-E \mathbf{I}\right)\right] \cdot \boldsymbol{\Psi} } & =0
\end{aligned}
$$

where, $\left[D_{2}\right]_{k, j}$ denotes the elements of the matrix representation of the second derivative. This matrix $\mathbf{D}_{2}$ is a square tridiagonal matrix with dimension $N_{z}^{2}$. The main diagonal elements are $-\frac{2}{h^{2}}$ and the upper and lower diagonals, $\frac{1}{h^{2}} . \mathbf{V}_{0}$ is a diagonal matrix with elements $V_{0}\left(z_{\underline{k}}\right)$. I, denotes the identity matrix which is constructed by the inner product $\left\langle\mathbf{e}_{\underline{k}} \mid \mathbf{e}_{j}\right\rangle=\delta_{\underline{k}, j}$. If we suppose the basis $\left\{\mathbf{e}_{\underline{k}}\right\}$ is orthonormal [89], then Eq. (2.51) becomes a standard eigenvectors and eigenvalues problem. Note that Eq. 2.51 has the form of a Lanczos matrix.

We numerically implement this method to compute all the bound states in 1D. Thus, we are able to remove the bound states from the final time-propagated wavefunction after the end of the laser pulse.

\section{Numerov method in $1 \mathrm{D}$ and scattering waves}

We use the Numerov method to compute the solutions of the TISE for the eigenwavefunctions, $\psi_{k}(z)$, with asymptotic momentum $k$. This will allow us to obtain the momentum spectrum of the final wavefunction after the interaction with a laser pulse. The Numerov method gives the wavefunction at a certain point $z_{\underline{k}}$ considering it is known at two points by following the formula [107]:

$$
\begin{aligned}
{\left[1+\frac{h^{2}}{12} f\left(z_{\underline{k}+1}\right)\right] \psi\left(z_{\underline{k}+1}\right)=} & 2\left[1-5 \frac{h^{2}}{12} f\left(z_{\underline{k}}\right)\right] \psi\left(z_{\underline{k}}\right) \\
& -\left[1+\frac{h^{2}}{12} f\left(z_{\underline{k}-1}\right)\right] \psi\left(z_{\underline{k}-1}\right)+\operatorname{Ord}\left(h^{6}\right),
\end{aligned}
$$

where, $f(z)$, is given by $f\left(z_{\underline{k}}\right)=2 \mu\left[E_{k}-V_{0}\left(z_{\underline{k}}\right)\right]$ with $E_{k}=\frac{k^{2}}{2 \mu}$. To build the numerical solution $\psi_{k}(z)$ for a given momentum $k$, this formula is computationally evaluated. The boundary conditions are: to match the numerical solution to the asymptotic function with $\psi_{k}(z) \approx \frac{1}{\sqrt{2 \pi}} e^{i k z+i \frac{Z}{k} \ln (2 k z)}$ (Coulomb wave) or $\psi_{k}(z) \approx \frac{1}{\sqrt{2 \pi}} e^{i k z}$ (plane wave) when $z \rightarrow \infty$ [108]. Numerically, this means that the two boundary values, $\psi_{k}\left(z_{N_{z}-1}\right)$, and $\psi_{k}\left(z_{N_{z}-2}\right)$, of the wavefunction can be evaluated at the limits of the grid $z$, i.e., $z_{N_{z}-1}=\left(N_{z}-1\right) \Delta z$ and $z_{N_{z}-2}=\left(N_{z}-2\right) \Delta z$. Finally, the numerical 
solution $\left\{\psi\left(z_{0}\right), \ldots, \psi\left(z_{N_{z}-3}\right), \psi\left(z_{N_{z}-2}\right), \psi\left(z_{N_{z}-1}\right)\right\}$ is computing by solving Eq. (2.52) for $\psi\left(z_{\underline{k}-1}\right)$.

Note that during the process of finding the solution, the normalization of the wavefunction is missing. In order to obtain a scattering waves, $\Psi_{k}(z)$, the computed continuum wavefunctions $\psi_{k}(z)$ are supposed to be re-normalized by a factor $c_{k}$ [108]:

$$
\Psi_{k}(z)=c_{k} \psi_{k}(z)
$$

where $c_{k}$ is evaluated by considering the scattering wave problem. The boundary conditions for this problem are illustrated in Fig. 2.1. The approaching incident wave, $\frac{1}{\sqrt{2 \pi}} e^{i k z}$, to the scattering center has a positive momentum $k>0$. If the incident wave comes from a far negative position, then, it will be split in two parts. The first part is the reflected wave, $\beta e^{-i k z}$, which is denoted by the black arrow in Fig. 2.1 and the second is the transmitted wave, $\alpha e^{i k z}$, (pink arrow in Fig. 2.1). The coefficients $\alpha$ and $\beta$ are the transmitted and reflected complex amplitudes, respectively.

When $z \rightarrow \infty$, let us assume that the numerical solution, $\Psi_{k}(z)$, can be constructed by considering a superposition of the incident and the reflected waves:

$$
\Psi_{k}(z)=c_{k} \psi_{k}(z) \approx \frac{1}{\sqrt{2 \pi}} e^{i k z}+\beta e^{-i k z} .
$$

Our main task is devoted to obtain the coefficient or normalization factor $c_{k}$ by solving above equation evaluated at $z_{0}=z_{\min }=-\frac{N_{z}}{2} \Delta z$. Eq. (2.54) shows that we have a single equation with two unknown coefficients $c_{k}$ and $\beta$. Then, another equation is needed. To this end we employ the continuum condition or criterion for the derivative of the wavefunction:

$$
c_{k} \dot{\psi}_{k}(z)=i k\left(\frac{1}{\sqrt{2 \pi}} e^{i k z}-\beta e^{-i k z}\right)
$$

Here, $\dot{\psi}_{k}(z)=\frac{d}{d z} \psi_{k}(z)$ denotes the position derivative of the wavefunction.

By solving the system of equations (2.54)-(2.55) for the coefficient $c_{k}$, we obtain:

$$
c_{k}=\left.\frac{2 k e^{i k z}}{k \psi_{k}(z)-i \dot{\psi}_{k}(z)}\right|_{z=z_{\min }}
$$


Scattering

Problem for $k>0$

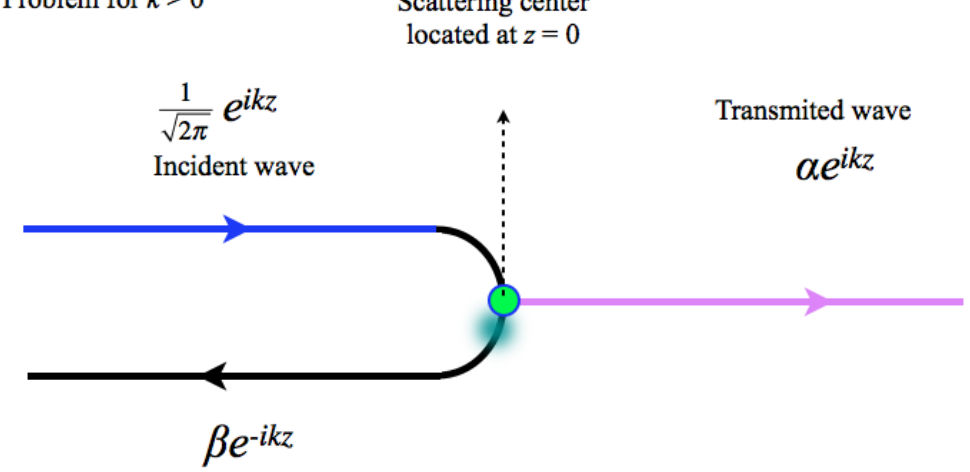

Reflected wave

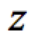

Figure 2.1: Scattering wave in 1D. This diagram represents by "arrows" the incident wave, $\frac{1}{\sqrt{2 \pi}} e^{i k z}$, which arrives to the scattering center (it is denoted by the green circle), the reflected wave, $\beta e^{-i k z}$, and the transmitted wave, $\alpha e^{i k z}$, for a scattering problem of momentum $k>0$. 
where $c_{k}$ is evaluated at the point $z_{\min }$. It is so, because the superposition of the incident wave and the reflected wave in Eq. (2.54) is defined for $z<0$ (see Fig. 2.1). We note that the outgoing solution is, $\alpha e^{i k z}+\beta e^{-i k z}$. It is worth to mention that when we refer to the asymptotic momentum $k$, this does not denote the kinetic momentum of the particle because the momentum $k$ comes form $E=\frac{k^{2}}{2}=\frac{\pi^{2}}{2}+V_{0}$, which is not the kinetic momentum $|\boldsymbol{\pi}|$.

Furthermore, to compute the scattering waves for negative asymptotic momentum $k<0$, we follow exactly the procedure given in reference [108]. Eq. (2.52) is numerically solved for $\psi\left(z_{\underline{k}+1}\right)$ by considering as the boundary values $\psi\left(z_{0}\right)=\frac{1}{\sqrt{2 \pi}} e^{i k z_{0}}$ and $\psi\left(z_{1}\right)=$ $\frac{1}{\sqrt{2 \pi}} e^{i k z_{1}}$. Here, $z_{0}=-\frac{N_{z}}{2} \Delta z$ and $z_{1}=\left(-\frac{N_{z}}{2}+1\right) \Delta z$ are the initial evaluated points. The normalization factor, $c_{k}$, is the same that the one defined in Eq. (2.56) but it is evaluated at $z=z_{\max }$.

\subsubsection{Electric-field of the laser pulses}

According to the dipole approximation the spatial dependence of the electric field is neglected. Then, we only consider the time dependence of the electric field, $\mathbf{E}(\mathbf{r}, t)=$ $\mathbf{E}(t)$. The electric field, $\mathbf{E}(t)$ and the vector potential $\mathbf{A}(t)$ will be defined in a temporal grid $t$ :

$$
t_{n}=t_{\min }+n \Delta t, \quad\left\{n=0, \ldots, N_{t}\right\}
$$

Here, $N_{t}$ is the total number of points, $t_{\min }$ denotes the initial time on the grid. $\Delta t$ is the time step or the sampling time. The frequency grid, $\omega$, is defined by:

$$
\omega_{n}=\omega_{\min }+n \Delta \omega, \quad\left\{n=0, \ldots, N_{t}\right\}
$$

where, $\omega_{\min }=-\frac{\pi}{\Delta t}$ and $\Delta \omega=\frac{2 \pi}{N_{t} \Delta t}$ are the Nyquist frequency and the frequency step, respectively.

The class timeobject.h defines a vector array which will contain the electric field $\mathbf{E}(t) \equiv\left(0, E_{y}(t), E_{z}(t)\right)$ and the vector potential $\mathbf{A}(t) \equiv\left(0, A_{y}(t), A_{z}(t)\right)$ components. This class also configures the routines of the fftw library [109], to compute the Fourier spectra of the above defined fields. It will be described with more details in Chapter 3. If the laser pulse is linearly polarized, $\mathbf{E}(t) \equiv\left(0,0, E_{z}(t)\right)$, then its form reads: 


$$
E_{z}(t)=E_{0}(t) \sin \left(\omega_{l}\left(t-t_{c}\right)+\varphi_{0}\right),
$$

where, $\varphi_{0}$, is the CEP, $E_{0}(t)$, is the envelope and, $\omega_{l}$, is the central or carrier frequency of the laser pulse. We will use different envelope shapes, e.g. $\sin ^{2}(t / \sigma), e^{-\frac{t^{2}}{\sigma^{2}}}$ and rectangular functions. The first one is the so-called $\sin ^{2}$ envelope:

$$
E_{0}(t)= \begin{cases}0, & \forall t<t_{a} \\ \varepsilon_{0} \sin ^{2}\left[\frac{\omega_{l}\left(t-t_{c}\right)}{2 N_{\mathrm{cy}}}\right], & \forall t_{a} \leq t \leq t_{b} \\ 0, & \forall t>t_{b}\end{cases}
$$

where, $t_{a}=t_{c}-\frac{\pi N_{\mathrm{cy}}}{\omega_{l}}$, and $t_{b}=t_{c}+\frac{\pi N_{\mathrm{cy}}}{\omega_{l}}$, are constants. $N_{\mathrm{cy}}$, denotes the number of cycles and $\varepsilon_{0}$, the peak of the electric field strength. In the second case the envelope is defined by a gaussian function $E_{0}(t)=\varepsilon_{0} \exp \left[-\left(t-t_{c}\right)^{2} / \sigma^{2}\right]$ with $\sigma=\frac{\pi N_{\mathrm{cy}}}{\omega_{l} \sqrt{\ln 2}}$. Note that $t_{c}$ denotes the centered time where the maximum of the envelope is located. In case of an elliptically polarized laser field the form of the pulse is given by:

$$
\mathbf{E}(t)=E_{0}(t)\left[\epsilon \xi \sin \left(\omega_{l}\left(t-t_{c}\right)+\varphi_{0}\right) \mathbf{e}_{y}+\xi \sin \left(\omega_{l}\left(t-t_{c}\right)+\varphi_{0}+\varphi_{\mathrm{rel}}\right) \mathbf{e}_{z}\right],
$$

where, $\xi=\frac{1}{\sqrt{1+\epsilon^{2}}}$ is a factor related with the ellipticity, and $\epsilon$ is the ellipticity parameter, e.g. if $\epsilon=0$, the laser is linearly polarized along $z$-direction, and circularly polarized when $\epsilon=1$ and $\varphi_{\text {rel }}=\frac{\pi}{2}$ (see for instance [94]). $\varphi_{\text {rel }}$ denotes the relative phases between the $y$ and the $z$-direction.

The vector potential of the laser field is computed by:

$$
\mathbf{A}(t)=-\int^{t} d t^{\prime} \mathbf{E}\left(t^{\prime}\right)
$$

This integral is numerically computed by the fourth-order Runge-Kutta method. We will give a fast description in Chapter 3 about the schemes to construct the laser pulses used in our simulations. defined electric field shapes of Eq. (2.59)-(2.60) and the corresponding vector potentials Eq. (2.61). Theses classes, timegrid.h, timeobject.h and laser.h, are all included in a library which is termed pulsesmaker [110].

\subsubsection{Time dependent evolution}

Once the initial wavefunction, $\Psi_{0}\left(\mathbf{r}, t_{0}\right)$, of the system is computed, our main task is focused in to obtain the time evolution of the wavefunction when the laser is acting on 
the atom or molecule. To this end, we propagate the initial wavefunction on time-steps by employing Eq. (2.35). The subsequent application of Eq. (2.35) will give us the wavefunction at each time step $\left\{t_{0}, t_{1}, \ldots, t_{n}, \ldots, t_{N_{t}}\right\}$, where the time-step $\Delta t$ or spacing time in the grid is uniform.

\subsubsection{Absorbing boundaries}

As a result of the laser-matter interaction, it is usual that the launched EWP to the continuum reaches the boundaries of the numerical box. Then, depending on the boundary conditions defined for the wavefunction, an "artificial reflections" might take place and introduce non-physical numerical interferences. This problem is solved by using absorbers at the boundary of the numerical box [111]. These absorbers are nothing else but a smooth function that multiplies the wavefunction $\Psi(\mathbf{r}, t)$ at the border of the grid.

Throughout this thesis we have implemented an analytical form for the absorbent $\cos ^{q}\left(\mathbf{k}_{0} \cdot \mathbf{r}\right)$. Here, $q$ is a parameter that controls the absorber shape. We use $q=1 / 6$ or $1 / 4$ and these parameters have been checked in order to obtain the most appropriate results. The parameter $\mathbf{k}_{0}$ along the $x$-direction is defined by $k_{x, 0, \mathrm{~L}}=\frac{\pi}{2 \Delta x_{\mathrm{L}}}$ and $k_{x, 0, \mathrm{R}}=$ $\frac{\pi}{2 \Delta x_{\mathrm{R}}}$. Here, $\Delta x_{\mathrm{R}}=x_{\max }-x_{b}$ is the length where the absorber will "act" on the wavefunction for all $x>0 . x_{b}$ is a fix point around the border of the grid on the $x$-axis in case of $x>0 . \Delta x_{\mathrm{L}}=x_{a}-x_{\min }$, denotes the length for $x<0$ where the absorber will be employed. $x_{a}$ defines the starting point of the absorber function in case of $x<0$. A similar analysis is performed for $y$ - and $z$-directions.

\subsubsection{Observables}

Once the wavefunction is computed at each numerical time $\left\{t_{0}, t_{1}, \ldots, t_{n}, \ldots, t_{N_{t}}\right\}$, we can obtain the observables required to analyze the problem under study. The observables that we compute are the expectation value of the position, the kinetic momentum, the potential energy, the kinetic energy values, amongst others. 
The components for the expectation value of the position $(\langle x\rangle,\langle y\rangle,\langle z\rangle)$ are:

$$
\begin{aligned}
& \langle x\rangle=\frac{\Delta \mathcal{V}_{\mathbf{r}}}{\mathcal{N}} \sum_{\underline{k}, \underline{j}, \underline{i}} \Psi^{*}\left(x_{\underline{i}}, y_{\underline{j}}, z_{\underline{k}}, t_{n}\right) x_{\underline{\underline{i}}} \Psi\left(x_{\underline{i}}, y_{\underline{j}}, z_{\underline{k}}, t_{n}\right), \\
& \langle y\rangle=\frac{\Delta \mathcal{V}_{\mathbf{r}}}{\mathcal{N}} \sum_{\underline{k}, \underline{j}, \underline{i}} \Psi^{*}\left(x_{\underline{i}}, y_{\underline{j}}, z_{\underline{k}}, t_{n}\right) y_{\underline{j}} \Psi\left(x_{\underline{i}}, y_{\underline{j}}, z_{\underline{k}}, t_{n}\right), \\
& \langle z\rangle=\frac{\Delta \mathcal{V}_{\mathbf{r}}}{\mathcal{N}} \sum_{\underline{k}, \underline{j}, \underline{i}} \Psi^{*}\left(x_{\underline{i}}, y_{\underline{j}}, z_{\underline{k}}, t_{n}\right) z_{\underline{k}} \Psi\left(x_{\underline{i}}, y_{\underline{j}}, z_{\underline{k}}, t_{n}\right),
\end{aligned}
$$

where, $\mathcal{N}$, denotes the norm of the wavefunction defined by:

$$
\mathcal{N}=\Delta \mathcal{V}_{\mathbf{r}} \sum_{\underline{k}, \underline{j}, \underline{i}} \Psi^{*}\left(x_{\underline{i}}, y_{\underline{j}}, z_{\underline{k}}, t_{n}\right) \Psi\left(x_{\underline{i}}, y_{\underline{j}}, z_{\underline{k}}, t_{n}\right),
$$

where, $\Delta v_{\mathrm{r}}=\Delta z \Delta y \Delta x$, is a finite volume element. It is important to point out that we implement also the position expectation values on the left and on the right, e.g. $\left\langle z^{(\mathrm{L} / \mathrm{R})}\right\rangle$ depending on the problem to be addressed. The implemented formula to compute these expectation values are similar to (2.62). The difference is the index limits in the sum for the calculated expectation value and the computed left-right norm.

The components of the kinetic momentum expectation values $\left(\left\langle p_{x}\right\rangle,\left\langle p_{y}\right\rangle,\left\langle p_{z}\right\rangle\right)$ are also computed by the same formulas defined in Eqs. (2.62). However, in order to perform the calculation of these momentum expectation values, we compute the FFT on $\Psi(\mathbf{r}, t)$ to obtain its momentum representation $\Psi(\mathbf{p}, t)$. Note we use the finite volume element in the momentum space defined as $\Delta \mathcal{V}_{\mathbf{p}}=\Delta p_{z} \Delta p_{y} \Delta p_{x}$.

The expectation value of the kinetic energy is computed by a similar way that the momentum value, but now we write:

$$
\langle T\rangle=\frac{\Delta \mathcal{V}_{\mathbf{p}}}{\mathcal{N}_{\mathbf{p}}} \sum_{\underline{k}, \underline{j}, \underline{i}} \Psi^{*}\left(p_{x_{\underline{i}}}, p_{y_{\underline{j}}}, p_{z_{\underline{k}}}, t_{n}\right) T_{\underline{i}, \underline{j}, \underline{k}} \Psi\left(p_{x_{\underline{i}}}, p_{y_{\underline{j}}, p_{z} \underline{k}}, t_{n}\right),
$$

with, $T_{\underline{i}, \underline{j}, \underline{k}}=\frac{1}{2}\left(p_{x_{\underline{i}}}^{2}+p_{y_{j}}^{2}+p_{\underline{z} \underline{k}}^{2}\right)$, is the spectral kinetic energy evaluated at the momentum point $\left(p_{x_{\underline{i}}}, p_{y_{\underline{j}}}, p_{z_{\underline{k}}}\right)$.

The expectation value of the potential energy, $\langle V\rangle$, is computed in the position space 
representation of the wavefunction by using:

$$
\langle V\rangle=\frac{\Delta \mathcal{V}_{\mathbf{r}}}{\mathcal{N}_{\mathbf{p}}} \sum_{\underline{k}, \underline{j}, \underline{i}} \Psi^{*}\left(x_{\underline{i}}, y_{\underline{j}}, z_{\underline{k}}, t_{n}\right) V_{\underline{i}, \underline{j}, \underline{k}} \Psi\left(x_{\underline{i}}, y_{\underline{j}} \underline{z}, \underline{k}, t_{n}\right),
$$

where, $V_{\underline{i}, j, \underline{k}}=V\left(x_{\underline{i}}, y_{\underline{j}}, z_{\underline{k}}\right)$, denotes the evaluated effective or potential energy fieldfree at the position point $\left(x_{\underline{i}}, y_{\underline{j}}, z_{\underline{k}}\right)$.

The expectation value of the total energy, $\langle E\rangle$, of the system is the sum of Eq. (2.64) and (2.65) and can be written as: $\langle E\rangle=\langle T\rangle+\langle V\rangle$.

Others interesting observables are the position and momentum distribution (electronic density). The position distribution can be easily computed and it is only the absolute square of the wavefunction in the position representation.

To compute the momentum distribution, we will focus ourselves in the continuum spectrum of the whole wavefunction. Let us say that the wavefunction $\Psi(\mathbf{r}, t)$ reads:

$$
\begin{aligned}
\Psi(\mathbf{r}, t) & =\Psi_{b}(\mathbf{r}, t)+\Psi_{c}(\mathbf{r}, t), \\
\Psi(\mathbf{r}, t) & =\sum_{n, l, m} c_{n}(t) \Phi_{n, l, m}(\mathbf{r})+\sum_{l^{\prime}, m^{\prime}} \int d E a(E, t) \Phi_{E, l^{\prime}, m^{\prime}}(\mathbf{r}),
\end{aligned}
$$

where, $n, l$ and $m$ are the principal quantum number, the total angular momentum number and the magnetic quantum number, respectively. $c_{n}(t)$, denotes the complex coefficient of the bound states corresponding to the Hilbert space of the field-free Hamiltonian $H_{0}$. These coefficients correspond to the discrete spectrum $\left\{\Phi_{n, l, m}(\mathbf{r})\right\}$ of $H_{0}$. On the another hand, $a(E, t)$, denotes the complex amplitude transition or coefficient for the electrons launched to the continuum written in the spectral base $\left\{\Phi_{E, l^{\prime}, m^{\prime}}(\mathbf{r})\right\}$ of $H_{0}$ with eigenenergy $E$.

Then, to compute numerically the momentum distribution, we can isolate the continuum part, second term on the right hand, in Eq. (2.66) by employing two different procedures. The first one is by removing out the discrete spectrum $\left\{\Phi_{n, l, m}(\mathbf{r})\right\}$ and projecting on plane waves.

In order to remove the bound states we use two methods: (a) to multiply the full wavefunction $\Psi(\mathbf{r}, t)$ by a mask function $f_{\text {Mask }}(\mathbf{r})$ and (b) to project out the bound states of the whole wavefunction.

(a) We isolate the continuum part of Eq. (2.66) by using the mask function method, i.e.

$$
\Psi_{c}(\mathbf{r}, t)=f_{\text {Mask }}(\mathbf{r}) \Psi(\mathbf{r}, t),
$$


with,

$$
f_{\text {Mask }}(\mathbf{r})= \begin{cases}0, & \forall r<r_{a} \\ e^{-\frac{\left(r-r_{b}\right)^{2}}{\sigma_{\mathrm{m}}^{2}}}, & \forall r_{a} \leq r \leq r_{b} \\ 1, & \forall r>r_{b}\end{cases}
$$

where, $r=\sqrt{x^{2}+y^{2}+z^{2}}$ denotes the radial position, $r_{a}$ is an inter-radial mask parameter, $r_{b}$ an outer-radial parameter and $\sigma_{\mathrm{m}}$ the length width of the gaussian mask. Then, an FFT is done on $\Psi_{c}(\mathbf{r}, t)$ to compute the instantaneous or final momentum distributions in the plane wave representation as in Eq. (??).

(b) We remove the bound states of the whole wavefunction $\Psi(\mathbf{r}, t)$ by projecting out the discrete states $\Phi_{n, l, m}(\mathbf{r}, t)$ :

$$
\Psi_{c}(\mathbf{r}, t)=\Psi(\mathbf{r}, t)-\sum_{n, l, m} c_{n}(t) \Phi_{n, l, m}(\mathbf{r})
$$

where the coefficients $c_{n}(t)$ are calculated according to:

$$
c_{n}(t)=\left\langle\Phi_{n, l, m} \mid \Psi(t)\right\rangle=\int d \mathbf{r} \Phi_{n, l, m}^{*}(\mathbf{r}) \Psi(\mathbf{r}, t) .
$$

Then, similarly to the method (a), by projecting the continuum part $\Psi_{c}(\mathbf{r}, t)$ on plane waves, the momentum distribution is obtained.

Another method to compute the momentum distribution is projecting on continuum waves at the end of the simulation, i.e., when the laser field is turned off. This method is only implemented in $1 \mathrm{D}$ by using the scattering waves described in Section 2.2.1.4 and by employing the procedure explained in Eqs. (2.53)-(2.56).

\subsubsection{Schrödinger equation in cylindrical coordinates}

An interesting numerical approach to solve the TDSE can be found for systems with cylindrical symmetry. For instance, when a laser pulse under the dipole approximation is employed to interact with the atomic system, is it known that the final excited states have a magnetic quantum number $m$ set to zero $(m=0)$. This is the case where the initial state of the system has a magnetic quantum number $m=0$, too.

Thus, the evolution of the wavefunction can be described by only two coordinates, namely the radius of the cylinder $\rho$ and the cylinder $z$-axis. From a computational point of view, this cylindrical symmetry is important because the number of operations is drastically reduced compared to a typical 3D system written in cartesian coordinates. 
This advantage allow us to compute faster numerical solutions of the TDSE for $\Psi(\rho, z, t)$ in cases where the laser pulse is linearly polarized. Furthermore, we can consider that $\Psi(\rho, z, t)$ is the solution of the TDSE in 3D without missin any effect of the azimuthal angle $\phi$.

The time-dependent Hamiltonian $H(t)$ in cylindrical coordinates are given by:

$$
\begin{aligned}
\Psi\left(\rho, z, t_{0}+\Delta t\right) & =e^{-i H\left(t_{0}+\frac{\Delta t}{2}\right) \Delta t} \Psi\left(\rho, z, t_{0}\right), \\
H(t) & =H_{\rho}+H_{z}(t),
\end{aligned}
$$

with,

$$
\begin{aligned}
H_{\rho} & =-\frac{1}{2 \rho} \frac{\partial}{\partial \rho}\left(\rho \frac{\partial}{\partial \rho}\right)+\frac{1}{2} V_{0}(\rho, z), \\
H_{z}(t) & =-\frac{1}{2} \frac{\partial^{2}}{\partial z^{2}}-i A_{z}(t) \frac{\partial}{\partial z}+\frac{1}{2} A_{z}^{2}(t)+\frac{1}{2} V_{0}(\rho, z) .
\end{aligned}
$$

We have separeted the Hamiltonian into two parts, $H_{\rho}$ and $H_{z}(t)$. One is related to the partial derivative along the $\rho$-axis and the other to the partial derivative of the Laplacian operator on the $z$-axis, respectively.

The Hamiltonian part $H_{z}$ contains the interaction of the laser field with the atomic system in the velocity gauge. This equation can be written in the length gauge and the laser-system coupling term will remain in the Hamiltonian $H_{z}$ :

$$
H_{z}(t)=-\frac{1}{2} \frac{\partial^{2}}{\partial z^{2}}+z E(t)+\frac{1}{2} V_{0}(\rho, z) .
$$

In the Chapter 3 we will explain the computational strategies employed to solve the TDSE in cylindrical coordinates.

\subsubsection{Crank-Nicolson methods in cylindrical coordinates}

According to the last Eqs. (2.70)-(2.74) and the split of the Hamiltonian $H$, the time evolution of the wavefunction can be expressed by:

$$
\Psi\left(\rho, z, t_{0}+\Delta t\right) \approx e^{-i H_{\rho} \frac{\Delta t}{2}} e^{-i H_{z}\left(t_{0}^{\prime}\right) \Delta t} e^{-i H_{\rho} \frac{\Delta t}{2}} \Psi\left(\rho, z, t_{0}\right),
$$

where, $t_{0}^{\prime}=t_{0}^{\prime}+\frac{1}{2} \Delta t$. Then, considering the Cayley formula for each exponential we can write: 


$$
\begin{aligned}
\Psi\left(\rho, z, t_{0}+\Delta t\right)= & \underbrace{\left(\frac{1-\frac{i \Delta t}{4} H_{\rho}}{1+\frac{i \Delta t}{4} H_{\rho}}\right)}_{(i i i)} \overbrace{\left(\frac{1-\frac{i \Delta t}{2} H_{z}\left(t_{0}^{\prime}\right)}{1+\frac{i \Delta t}{2} H_{z}\left(t_{0}^{\prime}\right)}\right)}^{(i i)} \underbrace{\left(\frac{1-\frac{i \Delta t}{4} H_{\rho}}{1+\frac{i \Delta t}{4} H_{\rho}}\right)}_{(i)} \Psi\left(\rho, z, t_{0}\right) \\
& +\operatorname{Ord}\left(\Delta t^{3}\right),
\end{aligned}
$$

and it is possible to evaluate them by using a Crank-Nicolson finite-differences scheme (see for instance [111]) in the grid points representation. The numerical accuracy of this Crank-Nicolson method scales with the $\Delta t^{3}$.

Eq. (2.76) is solved step by step in the sense that we obtain the numerical solution for two intermediate wavefunctions (or auxiliaries) $\chi(\rho, z)$ and $\Upsilon(\rho, z)$ and from these the wavefunction $\Psi\left(\rho, z, t_{0}+\Delta t\right)$. We define $\chi(\rho, z)$ to compute the first term $(i)$ in Eq. (2.76) as follows

$$
\left(1+\frac{i \Delta t}{4} H_{\rho}\right) \chi(\rho, z)=\left(1-\frac{i \Delta t}{4} H_{\rho}\right) \Psi\left(\rho, z, t_{0}\right)
$$

This equation can be solved independently for each position $z$. The action of the Hamiltonian $H_{\rho}$ (Eq. 2.72) on an arbitrary function $f=f(\rho, z)$ can be approximated with the finite element method by:

$$
\begin{aligned}
H_{\rho_{\underline{j}}} f\left(\rho_{\underline{j}}, z_{\underline{i}}\right) \approx & -\frac{1}{2} \frac{f\left(\rho_{\underline{j}+1}, z_{\underline{i}}\right)-2 f\left(\rho_{\underline{j}}, z_{\underline{i}}\right)+f\left(\rho_{\underline{j}-1}, z_{\underline{i}}\right)}{\Delta \rho^{2}} \\
& -\frac{1}{4 \rho_{\underline{j}} \Delta \rho}\left[f\left(\rho_{\underline{j}+1}, z_{\underline{j}}\right)-f\left(\rho_{\underline{j}-1}, z_{\underline{i}}\right)\right] \\
& +\frac{1}{2} V\left(\rho_{\underline{j}}, z_{\underline{\underline{j}}}\right) f\left(\rho_{\underline{j}}, z_{\underline{\underline{i}}}\right) .
\end{aligned}
$$

The term on the right hand side of Eq. (2.77), specifically the equivalent function $f(\rho, z)$, is completely known. The unknown wavefunction, $\chi(\rho, z)$, can be obtained by solving a tridiagonal linear system of equations for each $z_{\underline{i}}$ with the form:

$$
\mathbf{M}_{\underline{z}_{i}} \chi_{z_{i}}=\mathbf{b}_{z_{i}}
$$

Where, $\mathbf{b}_{z_{\underline{i}}}=\left\{\ldots, \Psi_{0}\left(\rho_{\underline{j}}, z_{\underline{i}}\right)-\frac{i \Delta t}{4} H_{\rho_{\underline{j}}} \Psi_{0}\left(\rho_{\underline{j}}, z_{\underline{i}}\right), \ldots\right\}$, is a vector array which contains 
the right hand side of Eq. (2.77) for each $z_{\underline{i}} . \mathbf{M}_{z_{i}}$ is a tridiagonal matrix. The elements of the main diagonal are $\mathbf{d}_{z_{i}}=\left\{\ldots, 1+\frac{i \Delta t}{4}\left[\frac{1}{\Delta \rho^{2}}+\frac{1}{2} V\left(\rho_{\underline{j}}, z_{\underline{i}}\right)\right], \ldots\right\}$ and the size of this diagonal is $N_{\rho} . \mathbf{d}_{z}^{(\text {up })}=\left\{\ldots, \frac{i \Delta t}{4}\left[-\frac{1}{2 \Delta \rho^{2}}-\frac{1}{4 \rho_{j} \Delta \rho}\right], \ldots\right\}$ denotes the elements of the first upper diagonal with respect to the main one and the size of this diagonal is $N_{\rho}-1$. Finally, the first lower diagonal is given by: $\mathbf{d}_{\underline{z}^{i}}^{(\text {down })}=\left\{\ldots, \frac{i \Delta t}{4}\left[-\frac{1}{2 \Delta \rho^{2}}+\frac{1}{4 \rho} \underline{j}^{\Delta \rho}\right], \ldots\right\}$.

Several routines can be implemented to solve Eq. (2.79). We have used the Thomas algorithm which implements a fast version to compute the solution of such a linear system of equations [112]. This algorithm has a number of operations of the order $O(N)$ instead of $O\left(N^{3}\right)$ required by the Gaussian elimination method. Then, by employing the Thomas method, we compute the solution $\chi_{z_{\underline{i}}}$ for each $z_{\underline{i}}$ and with it we obtain the first part ( $i)$ of Eq. (2.76).

Once the wavefunction $\chi(\rho, z)$ is calculated, the second term (ii) in Eq. (2.76) can be obtained by a similar procedure that in case of the term $(i)$. However, in the case (ii) the Laplace operator is solved along $z$-direction. Thus, to compute the term $(i i)$ we define the assistant wavefunction, $\Upsilon(\rho, z)$ as follows:

$$
\left(1+\frac{i \Delta t}{4} H_{z}\left(t_{0}^{\prime}\right)\right) \Upsilon(\rho, z)=\left(1-\frac{i \Delta t}{4} H_{z}\left(t_{0}^{\prime}\right)\right) \chi(\rho, z) .
$$

Where the action of the Hamiltonian term $H_{z}\left(t_{0}^{\prime}\right)$ on an arbitrary function $f=f(\rho, z)$ can be written as:

$$
\begin{aligned}
H_{z_{\underline{i}}} f\left(\rho_{\underline{j}}, z_{\underline{i}}\right) \approx & -\frac{1}{2} \frac{f\left(\rho_{\underline{j}}, z_{\underline{i}+1}\right)-2 f\left(\rho_{\underline{j}}, z_{\underline{i}}\right)+f\left(\rho_{\underline{j}}, z_{\underline{i}-1}\right)}{\Delta z^{2}} \\
& +\frac{-i A_{z}\left(t_{0}^{\prime}\right)}{2 \Delta z}\left[f\left(\rho_{\underline{j}}, z_{\underline{i}+1}\right)-f\left(\rho_{\underline{j}}, z_{\underline{i}-1}\right)\right] \\
& +\frac{1}{2}\left[V\left(\rho_{\underline{j}}, z_{\underline{i}}\right)+A_{z}^{2}\left(t_{0}^{\prime}\right)\right] f\left(\rho_{\underline{j}}, z_{\underline{i}}\right) .
\end{aligned}
$$

As the wavefunction $\chi(\rho, z)$ of Eq. (2.80) is already known, we write Eq. (2.80) in a system of linear equations for each $\rho_{\underline{j}}$ as:

$$
\mathbf{M}_{\rho_{\underline{j}}} \mathbf{\Upsilon}_{\rho_{\underline{j}}}=\mathbf{b}_{\rho_{\underline{j}}}
$$


where $\mathbf{b}_{\rho_{\underline{j}}}=\left\{\ldots, \chi\left(\rho_{\underline{j}}, z_{\underline{i}}\right)-\frac{i \Delta t}{4} H_{z_{\underline{i}}} \chi\left(\rho_{\underline{j}}, z_{\underline{i}}\right), \ldots\right\}$, is a vector array which contains the right hand side of Eq. (2.80) for each $\rho_{\underline{j}}$ and $\mathbf{M}_{\rho_{\underline{j}}}$ is a tridiagonal matrix similar to the one of Eq. (2.79). The elements of the main diagonal are $\mathbf{d}_{\rho_{j}}=\left\{\ldots, 1+\frac{i \Delta t}{4}\left[\frac{1}{\Delta z^{2}}+\right.\right.$ $\left.\left.\frac{1}{2}\left(V\left(\rho_{j}, z_{i}\right)+A_{z}^{2}\left(t_{0}^{\prime}\right)\right)\right], \ldots\right\}$ and the size of this diagonal vector is $N_{z}$.

$\mathbf{d}_{\rho_{\underline{j}}}^{(\mathrm{up})}=\left\{\ldots, \frac{i \Delta t}{4}\left[-\frac{1}{2 \Delta z^{2}}+\frac{i}{2 \Delta z} A_{z}\left(t_{0}^{\prime}\right)\right], \ldots\right\}$ denotes the elements of the first upper diagonal with respect to the main one and the size of this diagonal is $N_{z}-1$. Finally, the first lower diagonal is given by: $\mathbf{d}_{\rho_{\underline{j}}}^{(\text {down })}=\left\{\ldots, \frac{i \Delta t}{4}\left[-\frac{1}{2 \Delta z^{2}}-\frac{i}{2 \Delta z} A_{z}\left(t_{0}^{\prime}\right)\right], \ldots\right\}$. This diagonal vector $\mathbf{d}_{\rho_{\underline{j}}}^{\text {(down) }}$ has the same size of $\mathbf{d}_{\underline{\rho_{j}}}^{\text {(up) }}$. Thus, Eq. (2.82) can be solved by employing the same Thomas algorithm for the wavefunction $\boldsymbol{\Upsilon}_{\rho_{j}}$ at each $\rho_{\underline{j}}$.

Finally, to find the wavefunction $\Psi\left(\rho, z, t_{0}+\Delta t\right)$ we apply a similar algorithm that in Eq. (2.77). It is supposed that the wavefunction $\Upsilon(\rho, z)$ is known, thus:

$$
\left(1+\frac{i \Delta t}{4} H_{\rho}\right) \Psi\left(\rho, z, t_{0}+\Delta t\right)=\left(1-\frac{i \Delta t}{4} H_{\rho}\right) \Upsilon(\rho, z) .
$$

Here we follow the same steps described in Eq. (2.77) to obtain the unknown wavefunction $\Psi\left(\rho, z, t_{0}+\Delta t\right)$.

Furthermore, it is worth to mention that the spectral split operator method to integrate numerically the TDSE in cylindrical coordinates can be also implemented by solving:

$$
\Psi\left(\rho, z, t_{0}+\Delta t\right) \approx e^{-i T_{c} \Delta t / 2} e^{-i V\left(t_{0}^{\prime}\right) \Delta t} e^{-i T_{c} \Delta t / 2} \Psi\left(\rho, z, t_{0}\right)
$$

where, $T_{c}=-\frac{1}{2 \rho} \frac{\partial}{\partial \rho}\left(\rho \frac{\partial}{\partial \rho}\right)-\frac{1}{2} \frac{\partial^{2}}{\partial z^{2}}$ is the kinetic operator in cylindrical coordinates. This Eq. (2.84) is the same that Eq. (2.35) defined for cartesian coordinates (see Section 2.2.1.1), but now in cylindrical coordinates.

\subsubsection{Numerical aspects in cylindrical coordinates}

In this Section we will give a brief description of the numerical implementation of the TDSE in the cylindrical coordinates from a computational point of view. Similar to the case of the cartesian coordinates code, here we will define the cylindrical grid and the corresponding momentum space. We focus our efforts mainly in the description of the designed structure of the BALAS library [113]. 


\section{Position and momentum space}

The position space is defined by a pair of computational vector arrays $(\rho, z)$. Here, the axis are discretized by following the rule:

$$
\begin{aligned}
z_{i}=z_{\min }+\Delta z \underline{i} ; & \underline{i}=0, \ldots, N_{z}-1, \\
\rho_{\underline{j}}=\Delta \rho(\underline{j}+1 / 2) ; & \underline{j}=0, \ldots, N_{\rho}-1,
\end{aligned}
$$

where, $z_{\min }=-\frac{N_{z}}{2} \Delta z$ is the starting point in the $z$-axis, $\Delta z$ and $N_{z}$ denote the spacing grid and the total number of points along $z$-direction, respectively. The radial cylindrical coordinate $\rho$ is uniform in the sense that has a constant grid spacing $\Delta \rho$. The total number of points along this axis is $N_{\rho}$. It is important to point out that this axis has a starting point at $\Delta \rho / 2$ and not at zero. This is the case to avoid any divergence of the potential $V_{0}(\rho, z)=-\frac{Z}{\sqrt{\rho^{2}+z^{2}}}$ at the origin.

The HT is very well optimized in a radial $\rho^{\prime}$-axis which has a nonuniform grid step [114]. This nonuniform axis is defined within the points corresponding to the zeros (roots) of the Bessel function $J_{0}(x)$ of the first kind.

Let us denote those root points by $\alpha_{\underline{j}}$. Thus, the strategy that we will follow to compute the momentum distribution is to interpolate the wavefunction $\Psi(\rho, z, t)$ to a new nonuniform radial $\rho^{\prime}$-axis $\Psi\left(\rho^{\prime}, z, t\right)$. According to Guizar-Sicairos [114] this nonuniform radial axis reads:

$$
\rho_{\underline{j}}^{\prime}=\frac{\alpha_{j}}{\alpha_{N_{\rho}}} \rho_{\max } ; \quad \underline{j}=0, \ldots, N_{\rho}-1
$$

where $\alpha_{N_{\rho}}$ is the $N_{\rho}$-th root of the Bessel function $J_{0}(x)$ and $\rho_{\max }$ denotes the maximum of the numerical nonuniform radial grid.

The momentum grid is defined for the two momentum coordinates $\left(p_{\rho}, p_{z}\right)$. According to the Nyquist frequency, and similarly to the above defined momentum grid in cartesian coordinates (see Eq. (2.41), the momentum axis along the $z$-direction is:

$$
p_{z_{i}}=p_{\text {zmin }}+\Delta p_{z} \underline{i} ; \quad \underline{i}=0, \ldots, N_{z}-1,
$$

where $p_{\text {zmin }}=-\frac{\pi}{\Delta z}$ is the minimum momentum on the grid and $\Delta p_{z}=\frac{2 \pi}{N_{z} \Delta z}$ denotes the momentum sampling, respectively.

According to reference [114], the radial momentum axis $p_{\rho}$ is nonuniform and it is given 
by:

$$
p_{\rho_{j}}=\frac{\alpha_{\underline{j}}}{\rho_{\max }} ; \quad \underline{j}=0, \ldots, N_{\rho}-1
$$

We have already described all the numerical requirements to implement the position and momentum grids in cylindrical coordinates. In the next Section we will define the time evolution of the system.

\section{Wavefunction, potential and system time evolution}

The wavefunction $\Psi(\rho, z)$ of the system is computationally defined in a complex vector array, $\boldsymbol{\psi}$ of size $N_{\mathrm{T}}=N_{\rho} N_{z}$.

Similar to the strategy followed in cartesian coordinates to arrange the wavefunction (see text about Eq. (2.42)), we will put the elements of the wavefunction $\Psi(\rho, z)$ in a computational vector array $\psi$. The first row, corresponding to the elements:

$$
\left\{\Psi\left(\rho_{0}, z_{0}\right), \ldots, \Psi\left(\rho_{0}, z_{i}\right), \ldots, \Psi\left(\rho_{0}, z_{\mathrm{N}_{z}-1}\right)\right\}
$$

is put on the first $N_{z}-1$ memory positions of the vector $\psi$. Then, the next row, which corresponds to the elements $\left\{\Psi\left(\rho_{1}, z_{0}\right), \ldots, \Psi\left(\rho_{1}, z_{i}\right), \ldots, \Psi\left(\rho_{1}, z_{N_{z}-1}\right)\right\}$, is put between the $N_{z}$ and $2 N_{z}-1$ memory positions of $\boldsymbol{\psi}$. By using this logical sequence all the remaining elements $\Psi\left(\rho_{j}, z_{i}\right)$ are arranged in $\boldsymbol{\psi}$.

The field-free potential well $V_{0}(\rho, z)$, which describes the interaction between the electron and the nucleus core, is also defined as a vector array pot member of the class waveUniform2D.h. This variable $\operatorname{pot}\left[N_{\mathrm{T}}\right]$ has the same size $N_{\mathrm{T}}$. The elements of the potential energy well $V_{0}\left(\rho_{j}, z_{i}\right)$ are arranged in the vector array pot by following the same procedure that the wavefunction $\Psi(\rho, z)$.

Furthermore, to obtain the ground state $\Psi_{0}(\rho, z)$ of a potential under study, we will use the imaginary time reverse evolution method described in Section 2.2.1.4 and specified in Eq. (2.47). This calculation can be performed by employing the described steps in Section 2.2.2.1 and considering that the vector potential of the system is set to zero, i.e., $A_{z}(t)=0$.

The time evolution of the initial wavefunction $\Psi_{0}(\rho, z)$ at the time $t_{0}$, to the electron wavefunction $\Psi(\rho, z, t)$ at the time $t$ and considering the action of the laser pulses, is performed by using the Crank-Nicolson method 2.2.2.1. This propagation of the wavefunction is supposed to be in real time.

Once the time evolution of the wavefunction of the system is known along the grid time 
$\left\{t_{0}, \ldots, t_{n}, \ldots, t_{N_{t}}\right\}$, we can compute the same observables as those calculated in Section 2.2.1.8 but in cylindrical coordinates. The expectation values of the position $(\langle\rho\rangle,\langle z\rangle)$ are computed by:

$$
\begin{aligned}
& \langle\rho\rangle=\frac{1}{\mathcal{N}} \sum_{\underline{j}, \underline{i}} \rho_{\underline{j}} \Psi^{*}\left(\rho_{\underline{j}}, z_{\underline{i}}, t_{n}\right) \rho_{\underline{j}} \Psi\left(\rho_{\underline{j}}, z_{\underline{i}}, t_{n}\right) \Delta \rho \Delta z, \\
& \langle z\rangle=\frac{1}{\mathcal{N}} \sum_{\underline{j}, \underline{i}} \rho_{\underline{j}} \Psi^{*}\left(\rho_{\underline{j}}, z_{\underline{\underline{i}}}, t_{n}\right) z_{\underline{i}} \Psi\left(\rho_{\underline{j}}, z_{\underline{i}}, t_{n}\right) \Delta \rho \Delta z .
\end{aligned}
$$

Where the norm $\mathcal{N}$ in cylindrical coordinates reads:

$$
\mathcal{N}=\sum_{\underline{j}, \underline{i}} \rho_{\underline{j}} \Psi^{*}\left(\rho_{\underline{j}}, z_{\underline{i}}, t_{n}\right) \Psi\left(\rho_{\underline{j}}, z_{\underline{i}}, t_{n}\right) \Delta \rho \Delta z
$$

The expectation value of the kinetic energy $\left\langle T_{c}\right\rangle$, corresponding to the operator $T_{c}=$ $\frac{p_{\rho}{ }^{2}}{2}+\frac{p_{z}{ }^{2}}{2}$, can be computed by the finite element method. Here, we only define the general form of this expectation value:

$$
\left\langle T_{c}\right\rangle=\frac{1}{\mathcal{N}} \sum_{\underline{j}, \underline{i}} \rho_{\underline{j}} \Psi^{*}\left(\rho_{\underline{j}}, z_{\underline{i}}, t_{n}\right) T_{c}\left(\rho_{\underline{j}}, z_{\underline{j}}\right) \Psi\left(\rho_{\underline{j}}, z_{\underline{i}}, t_{n}\right) \Delta \rho \Delta z,
$$

The expectation value of the potential energy $\left\langle V_{0}\right\rangle$ in cylindrical coordinates has the same definition that Eq. (2.92). We only need to replace the operator $T_{c}$ by $V_{0}$.

Once the kinetic and potential energies expectation values are computed, we can obtain the total energy expectation value by using $\langle E\rangle=\left\langle T_{c}\right\rangle+\left\langle V_{0}\right\rangle$.

Other important observables are the electron position distribution (or electron density) and the electron momentum distribution. The electron position density is easily computed by using $\mathrm{S}_{e}(\rho, z, t)=\rho|\Psi(\rho, z, t)|^{2}$.

The momentum distribution of the continuum wavefunction or wavepacket $\Psi_{c}(\rho, z, t)$ will be calculated in cylindrical coordinates into two steps. First of all, we compute the continuum wavepacket in the position representation $\Psi_{c}(\rho, z, t)=f_{\text {Mask }}(\rho, z) \Psi(\rho, z, t)$ by filtering out the bound states out of the whole wavefunction $\Psi(\rho, z, t)$. We perform this filtering process by employing a smooth mask function $f_{\text {Mask }}(\rho, z)$ around the box region $\left(0,-z_{\text {mask }}\right)$ and $\left(\rho_{\text {mask }}, z_{\text {mask }}\right)$ where the bound states are located. Here, $\rho_{\text {mask }}$ and $z_{\text {mask }}$ are positive real numbers. Similar steps are described in Eq. (2.67) for the cartesian coordinates case. 
Second, once the continuum wavepacket, $\Psi_{c}(\rho, z, t)$, is obtained, the momentum distribution or electron density $\mathrm{S}_{e}\left(p_{\rho}, p_{z}, t\right)=\left|\Psi_{c}\left(p_{\rho}, p_{z}, t\right)\right|^{2}$ is computed by projecting on the corresponding plane wave in cylindrical coordinates. Here, $\Psi_{c}\left(p_{\rho}, p_{z}, t\right)$ denotes the momentum representation of the complex wavepacket $\Psi_{c}(\rho, z, t)$. The mathematical procedure to obtain $\Psi_{c}\left(p_{\rho}, p_{z}, t\right)$ reads:

$$
\Psi_{c}\left(p_{\rho}, p_{z}, t\right)=\sqrt{2 \pi} \iint \rho J_{0}\left(p_{\rho} \rho\right) e^{-i p_{z} z} \Psi_{c}(\rho, z, t) d \rho d z
$$

where, $J_{0}\left(p_{\rho} \rho\right)$, is the Bessel function of zero order [114]. It is important to mention that in our scheme we use the Bessel function of zero order. This is the case because the laser is linearly polarized along $z$-axis. This means that the magnetic momentum $m$ is zero. In the case the laser is circularly polarized, the Bessel function is not only of zero order and has to be replaced by $J_{m}\left(p_{\rho} \rho\right)$.

On the other hand, a FFT of the wavefunction is computed on the cylinder $z$-axis. It is so, to obtain the momentum distribution component along such a $z$-direction.

Note that the integral on $\rho$-axis denotes a Hankel transform which is defined by:

$$
g\left(p_{\rho}\right)=2 \pi \int \rho J_{0}\left(p_{\rho} \rho\right) f(\rho) d \rho
$$

where, $f\left(p_{\rho}\right)$, is the function which will be transform. We do HT of the wavefunction $\Psi_{c}(\rho, z)$ on $\rho$-direction for each $z$.

A "fast" implementation of this transform is described in [114]. We have numerically implemented this fast Hankel transform in two structures classes, namely arrai .h and HankelMatrix.h. These classes are mentioned and fast explained in Chapter 3.

This numerical implementation of the Hankel transform requires to be computed along a nonuniform $\rho^{\prime}$ radial grid Eq. (2.86). It is so, because the designed Hankel transform is determined by the evaluation of the Bessel function $J_{0}\left(p_{\rho} \rho\right)$ at its root points $\alpha_{j}$. Then, we will interpolate the evaluated wavefunction $\Psi(\rho, z, t)$ from the uniform radial $\rho$ grid to the nonuniform $\rho^{\prime}$-axis. Note that the interpolation is exclusively performed along the radial axis. We take advance of the MKL routines [115] to do this interpolation. Once the new interpolated wavefunction $\Psi\left(\rho^{\prime}, z, t\right)$ is obtained, we transform it according to our numerical implementation of the integral in Eq. (2.94) (for detail see [114]). 


\subsubsection{Numerical integration: ionization transition amplitude}

We will use the Runge-Kutta to integrate numerically the solution of the SFA equation for the direct ionization mediated by an attosecond pulses in presence of a weak IR laser field. The ionization of an atom or molecule under the action of a strong laser pulse is described by the complex transition amplitude Eq. (2.29). Through this thesis the main goal within the SFA approach is to describe the photoionization led by an XUV attosecond pulse in the presence of a weak IR laser field. Thus, our main task is focused into calculate the integral of the Eq. (2.31). The numerical solution of this integral is by far a no trivial problem. This is the case because of two main aspects: the first, it is a double nested integral in time (see the phase factor in Eq. (2.31)). Second, the integrand is highly oscillatory due to the presence of a phase factor [50].

Several approximation methods have been developed to solve this integral, e.g. the saddle point in time $[50,94]$ and converting this integral to a differential form. The latter can be done just by taken the derivative $a(\mathbf{p}, t)$ with respect to the time $t$ [116]. This last method simplifies this integral Eq. (2.31) to an ordinary non homogeneous differential equation given by:

$$
\frac{d a(\mathbf{p}, t)}{d t}+i \frac{\left(\mathbf{p}+\mathbf{A}_{\mathrm{L}}(t)\right)^{2}}{2} a(\mathbf{p}, t)=i \mathbf{E}_{\mathbf{X}}(t-\tau) \cdot \mathbf{d}\left(\mathbf{p}+\mathbf{A}_{\mathrm{L}}(t)\right) e^{i I_{p}\left(t-t_{0}\right)}
$$

Note that this differential equation can be easily integrated by a Runge-Kutta method of fourth-order. In addition, the momentum $\mathbf{p}$ in the complex amplitude $a(\mathbf{p}, t)$ is just a parameter for such an equation. This means that we can be parallelize Eq. (2.95) by using MPI or OpenMP paradigms to speed up our numerical calculations.

\subsubsection{Runge-Kutta method}

In numerical analysis the fourth-order Runge-Kutta method is employed to solve ordinary differential equations [103]. Let us assume that an initial value problem is specified by the following conditions:

$$
y^{\prime}=f(t, y), \quad y\left(t_{0}\right)=y_{0},
$$

where, $y(t)$, denotes the unknown function of time $t$ which we will solve. $y^{\prime}(t)$ is the time derivative of $y$ which is a function of $t$ and $y$. It is important to note that at the initial time it is supposed that the function $y\left(t_{0}\right)=y_{0}$ is known. The fourth-order 
Runge-Kutta formula are defined according to:

$$
\begin{aligned}
k_{1} & =h f\left(t_{n}, y_{n}\right), \\
k_{2} & =h f\left(t_{n}+\frac{h}{2}, y_{n}+\frac{k_{1}}{2}\right), \\
k_{3} & =h f\left(t_{n}+\frac{h}{2}, y_{n}+\frac{k_{2}}{2}\right), \\
k_{4} & =h f\left(t_{n}+h, y_{n}+k_{3}\right), \\
y_{n+1} & =y_{n}+\frac{k_{1}}{6}+\frac{k_{2}}{3}+\frac{k_{3}}{3}+\frac{k_{4}}{6}+O \operatorname{rd}\left(h^{5}\right) .
\end{aligned}
$$

Here, $h=\Delta t$ denotes the time step and $t_{n}=t_{0}+n h$ define the total time axis. This method requires four evaluations of the right-hand side per step $h$. This method is high accurate, on the order of $\operatorname{Ord}\left(h^{5}\right)$. We are interested in employing this Runge-Kutta method to solve numerically Eq. (2.95). We have also built a library in $\mathrm{C} / \mathrm{C}++$ to implement the SFA complex ionization amplitude.

This problem can be split in three pieces. (i) The calculation of the bound-free dipole transition matrix element. In the systems which are employed throughout this thesis, all the dipole matrix element under the SFA approach have closed analytical expressions. (ii) The electric fields and the vector potentials and (iii) The computation of the transition complex amplitude $a_{\mathbf{p}}(t)$. Our algorithm employs the PULSESMAKER library [110].

\subsubsection{Momentum grid definition in SFA}

As the complex transition amplitude $a(\mathbf{p}, t)$ is parametrized along the momentum axis, we can define the momentum grid as follows:

$$
\begin{array}{ll}
p_{x, \underline{i}}=-p_{\mathrm{xmax}}+\underline{i} \Delta p_{x} ; & \underline{i}=0, \ldots, N_{x}-1, \\
p_{y, \underline{j}}=-p_{\mathrm{ymax}}+\underline{j} \Delta p_{y} ; & \underline{j}=0, \ldots, N_{y}-1, \\
p_{z, \underline{k}}=-p_{\mathrm{zmax}}+\underline{k} \Delta p_{z} ; & \underline{k}=0, \ldots, N_{z}-1,
\end{array}
$$

where, $\left(p_{\text {xmax }}=\frac{N_{x}}{2} \Delta p_{x}, p_{\text {ymax }}=\frac{N_{y}}{2} \Delta p_{y}, p_{\text {zmax }}=\frac{N_{z}}{2} \Delta p_{z}\right)$ are the maximum momentum for each particular $(x, y, z)$-direction. Here, $\left(\Delta p_{x}, \Delta p_{y}, \Delta p_{z}\right)$ is the grid momentum spacing on each direction.

In this Chapter we have defined all the basic Physical, Mathematical and Numerical tools which will be utilized throughout this thesis. These tools will allow us to 
address the research questions formulated in the introduction and in particular how it is possible to employ an SPIDER scheme to extract structural information of the target. Furthermore, we will employ these tools to study the fundamental delay in photoemission process by utilizing the atttosecond streaking techniques. 


\section{3}

\section{Software design}

\subsection{Introduction}

As described in the previous Chapter 2, the main theoretical tool in this thesis is the numerical integration of the TDSE. This is made through the use of several simulation codes.

The numerical effort and the complexity to solve the TDSE on a grid grows exponential with the number of degrees of freedom. The more degrees of freedom are included, the more demanding the employed computational resources are. Therefore, it is necessary to develop codes with the propose of handling the complexity of the physics problems.

Traditionally, physicists write a specific code to simulate a particular physical phenomenon. When they address a similar problem, commonly a new code is written based on the previous one. In such a case, two practical difficulties arise.

First, it is hard to scale such a code. As the physical problem becomes more complex, i.e., involving high dimensionality $(3 \mathrm{D}, 4 \mathrm{D}$, etc.), the simulations are more demanding and therefore a design is required to deal with computational complexity.

Second, the messy codes are difficult to change and every modification requires a long time. This huge effort reduces the time that we can be dedicated to the actual physical problem.

The software development has an important role in this thesis. Beyond the traditional development of a single code, a designed group of libraries is carried out through this paper. These libraries are utilized to study a variety of ultrafast and strong field physics phenomena.

Two important aspects to take into account in the development of an efficient simulation 


\section{DESIGN STRATEGIES FOR THE CODES}

program are identified. First, the choice of the algorithms to simulate the physical problem with accurate results. Second, it is required to build an efficient code or software to carry out simulations in the facilities with High Performance Computing.

Ruiz and Becker have had a long experience in these kind of simulation strategies and they have found an approach to handle them. The aim of this method is to develop a simulation software based on simple design strategies. The first software of this kind was the SimLab developed in the Max Planck Institute for Complex Systems by Ruiz and Becker. Ruiz designed and developed QFISHBOWL [117] and ASSAM [118]. The author of this thesis and De La Calle have grown up these two libraries with new routines and classes. Following a similar strategy to the ones in QFISHBOWL, the author of this thesis and Ruiz have developed and designed the BALAS code [113]. Furthermore, the author of this thesis has designed and developed two new codes: the PULSESMAKER [110] and QSPHERE [119]. All of these libraries or codes have been implemented with the aim of simulating the physical problems regarding the research questions mentioned in Section 1.4.

This Chapter is dedicated to present the Design Strategy which is employed to develop our libraries. The Chapter also describes quickly how these libraries are implemented and can be used as a Simulation Lab.

Throughout the next Sections we will describe the design strategies for the codes, in particular:

(i) Define the structure of the library based on the physics concepts,

(ii) Separate the numerical algorithms from the library,

(iii) Fast-ideas cycle, from sketches to full scale simulations.

\subsubsection{Library structures according to physics concepts}

To describe a physical phenomenon, physicists define a set of "objects", or quantities, i.e, electron position, electron momentum, electron energy, electromagnetic field, etc., regarding the particular phenomenon that they are interested to study. We keep this language in the simulation codes with the motivation to define a correspondence between the physical "objects" and computational ones.

This design strategy is based on using the logic and the magnitudes of the quantum theory to guide the construction of a simulation software. The first step towards such a goal is to identify several structures of the theory as objects, but here in a computational context. 
The language $\mathrm{C}++$ has a class and object oriented programming paradigm which enables the construction of classes. We can represent structures made of several variables and methods (or routines). The main advantage of $\mathrm{C}++$ is the feasibility to split a computational problem in small classes. A class is a user defined type or data structure declared with keyword class that has data and routines. The access to those variables and routines is governed by the three access specifiers private, protected or public (by default the access to members of a class is private).

As an example, Fig. 3.1 shows an schematic representation of how to implement this design strategy.

a)

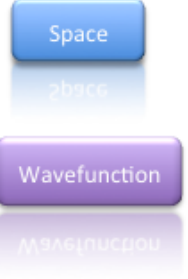

c)

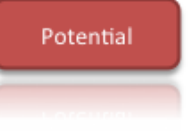

d)

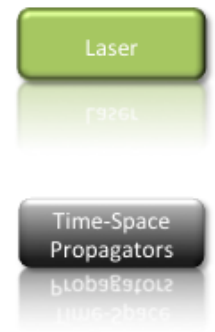

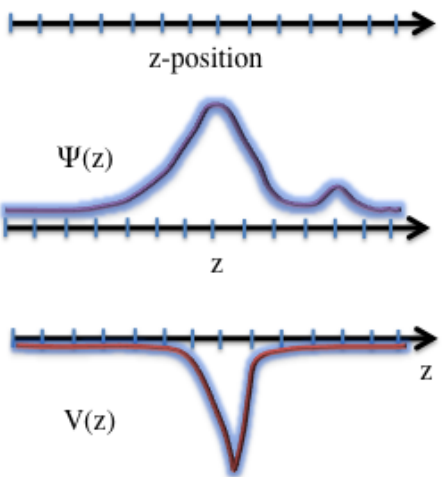

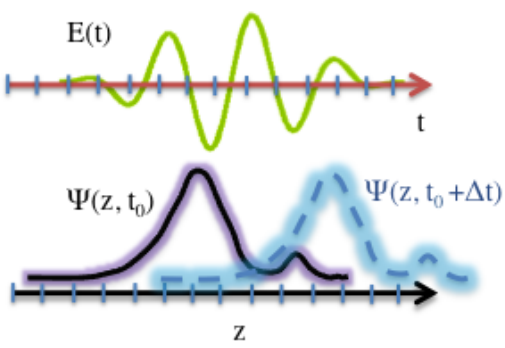

Figure 3.1: Designing code within physics concepts. In this picture, the space dependence on the wavefunction, potential and evolution (or propagator) operators is split. Then, this separation allows us to build computational classes in $\mathrm{C}++$ with the features of these physical concepts.

In Quantum Mechanics, the calculation of the temporal and spatial evolution of the wavefunctions is one of the main physical problems. This picture Fig. 3.1 identifies the different physical "components" or in analogy the computational objects, which are required to compute the evolution of the wavefunction. Five steps to identify physics objects as computational ones are depicted in Fig. 3.1. 


\section{DESIGN STRATEGIES FOR THE CODES}

First, (a), the spatial region where the wavefunction "lives" is required. From a computational viewpoint, this is described by a grid class which sets the spatial features of the simulations. The grid class (space: position coordinates and momentum coordinates) is illustrated by the soft-blue box in the Fig. 3.1.

Second, (b), the wavefunction lives in the defined spatial region. This computational memory space is described by a wavefunction class. This wavefunction also has the characteristics of the spatial grid.

Third, (c), the potentials which defines the field-free Hamiltonian are implemented in a different class which is also defined on the same spatial grid of the simulation.

As a fourth step, (d), the electric field or vector potential of the external laser field is described in a separated class which defines the parameters of the laser pulse: the time axis, the envelope, the carrier frequency, the CEP, etc.

The external field is a common class in all the simulations related to ultrafast and strong field problems. Thus, we will dedicate the Section 3.2 to describe how this library was built.

Fifth, (e), all of these physical structures or computational classes are required to compute the evolution of a wavefunction. Therefore, the physics of laser-matter interactions can be simulated. Before, during and after the simulations, some observables can be recorded to describe the effects of different external fields in the physical system under study.

The previous analogies give rise to the organization of the code in libraries where the classes and their methods (routines) are defined. To use them, it is only necessary to create small programs or "scripts" where the libraries are included.

These small scripts produce a functionality similar to the MATLAB Software. The scripts use prefabricated routines to compute a specific tasks. For instance, in MAT$\mathrm{LAB}$, it is enough to write $\mathrm{ft}(\mathrm{f})$ for computing the Discrete Fourier Transform instead of implementing a new DFT code each time.

The construction of our libraries regarding these physical concepts allows us to include the logic of the theory in the simulations.

\subsubsection{Basic numerical tools splitting}

The second designed strategy is referred to the separation of the numerical algorithms from the libraries or codes.

All the numerical methods such as Crank-Nicolson or split-operator spectral methods used in these libraries are based on generic numerical tools. Fast Fourier Transform 
(FFT), Linear algebra algorithms (matrix inversion or matrix product), Fast Legendre Transform, etc., are some examples of generic algorithms.

These algorithms are available as numerical libraries for different architectures.

Our implementation is designed with the aim to prevent unnecessary drastic changes of the libraries, if a new version of these numerical routines appears as a result of the fast evolution of the computational technologies. This flexibility can be illustrated by a flow diagram in Fig. 3.2.

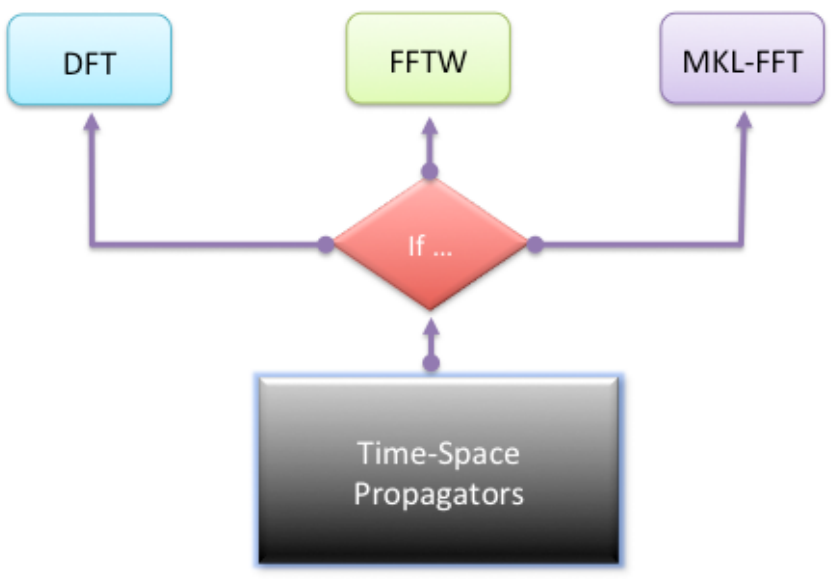

Figure 3.2: Software architecture: flexible design. This flow diagram illustrates the steps followed by our separation of the basic numerical routines from the library or main code. In particular, we depict how a library can use the Discrete Fourier Transform (DFT), the Fast Fourier Transform in the West (FFTW), and the MKL optimization of the FFT, to compute the split-operator technique described in Section 2.2.1.1.

For instance, if the computational technology achieves a faster FFT on future, than the nowadays numerical library FFTW, our code can easily be modified to use it.

These procedures can also be done with computational paradigms (parallel programming schemes). For instance, different numerical libraries for the linear product of large matrices are implemented. We can then make use of the speed up coming from the use of a large number of cores, e.g., in a chip using the OpenMP implementation or the optimized BLAS routines for the Intel chips in the MKL numerical library. This means that these libraries can be used for a long time even if new and more efficient implementations or computational paradigms appear. 


\subsubsection{Cycle of Ideas}

Another important design strategy is related to speed up the development of ideas. The concept is to produce quick tests of the physical problems which we can study.

The "cycle" of a particular physical problem or idea can be usually addressed by testing a reduced version of the problem before trying the actual problem in its full scale. The cycle of ideas is represented in a diagram in Fig. 3.3.

(i)

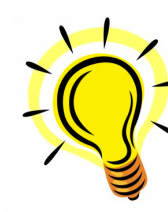

(ii)

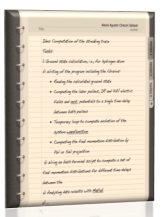

(iii)

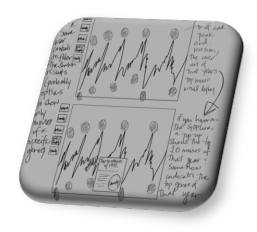

(iv)
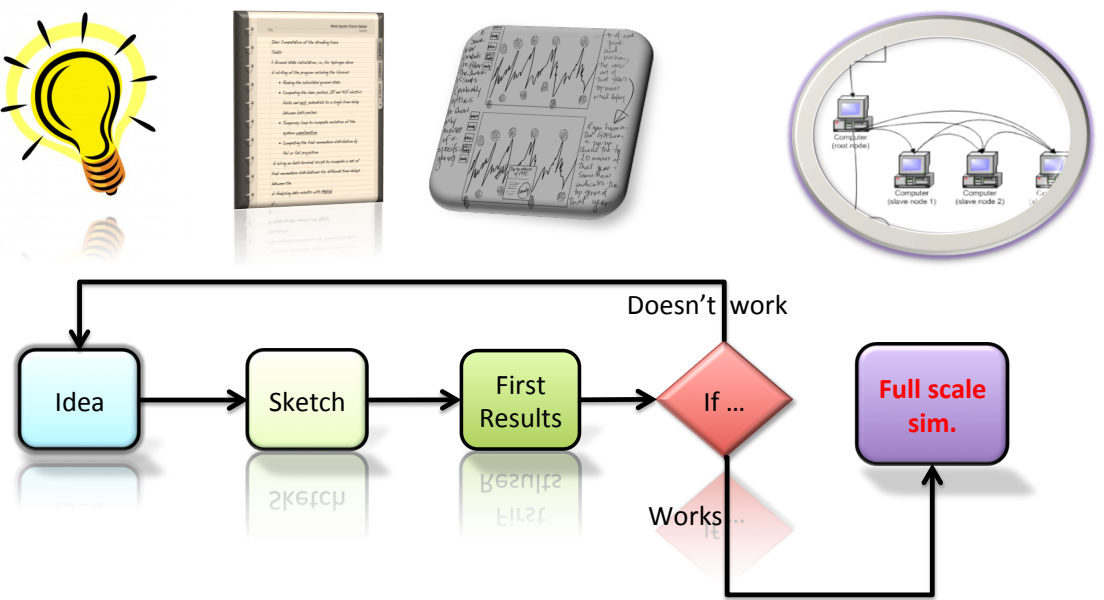

Figure 3.3: Cycle of ideas. The diagram depicts the general way to test a physical idea from a numerical point of view. ( $i$ ) A general idea (or research question) about the physical problem is conceived, ( $i i$ ) the sketch of how to address it is developed, (iii) the preliminary results about the research question are obtained and $(i v)$ the scale on dimensionality or scan on parameters is performed, if the preliminary results demonstrate that the idea works.

Once the computational software in our libraries is completed and tested, the actual physical problems can be addressed. For instance, if we study the emitted radiation from the interaction of a strong laser field with an atom, it is important to produce the first results in a very little amount of time.

Some specific parameters require to be well characterized in order to reach good convergence of our results, e.g. the grid size (length) of the problem, the integration time step $\Delta t$, the position step $\Delta z$, the positions of the absorbing boundary functions, etc. Thus, it is suitable to perform a $1 \mathrm{D}$ calculation in order to optimize the grid size and to analyze the fundamental physics of the problem before addressing a high dimensionality 
problem, e.g. in $2 \mathrm{D}$ or $3 \mathrm{D}$.

If a long time is required to produce a new code for different physical systems, then the cycle of ideas becomes too long and not adequate to develop a research program. Our libraries allow us to test many ideas in a very short amount of time.

These evaluation tests are quite important, because several ideas can quickly address with a single part of software. This helps us to focus on the physical phenomenon rather than the code.

As an application of our design strategies, the next Sections will be dedicated to describe PULSESMAKER (Section 3.2), QFISHBOWL (Section 3.3), BALAS (Section 3.4), and QSPHERE (Section 3.5) libraries.

In the following Section 3.2, we will describe how PULSESMAKER library has been implemented with the purpose to build a single pulse and a delayed train of pulses with arbitrary feasures in the time domain.

\subsection{PULSESMAKER}

PULSESMAKER is a library written in $\mathrm{C} / \mathrm{C}++$ with a programming structure based on classes and objects [110]. It is built with the aim of generating laser pulses for simulation of our physical problems. This library can be used in any of the other libraries described here, i.e., QFISHBOWL, BALAS and QSPHERE. In addition, the library might be used as a stand alone library as well.

The library is designed to create numerically single pulses, train of pulses or a set of pulses with arbitrary characteristics. It is possible to define as many pulses as needed with different delay, intensity, carrier frequency, time duration, polarization, envelope, etc. The resulting external field pulse can be used for all kinds of numerical simulations of laser-matter interaction within the dipole approximation.

The PULSESMAKER library consists of four different classes which are: cartesian.h, timegrid.h, timeobject.h and laser.h in Fig. 3.4 we illustrate how the design of PULSESMAKER is done and its relation with the standard $\mathrm{C} / \mathrm{C}++$ libraries.

The diagram explains the logical order of the library: first, a constant class, which contains all the physical constants required along the logical process to solve the TDSE. This class, defines also all the required standard $\mathrm{C} / \mathrm{C}++$ libraries, standard variables and routines of $\mathrm{C} / \mathrm{C}++$. These are, for example, stdio.h, iostream, math, vector, etc. We also use the the General Public License (GNU) fftw3.h library [109].

Then, the general diagram shows the timegrid.h, the timeobject.h, and finally the laser.h classes. PULSESMAKER has become one of the main pieces of the QFISH- 


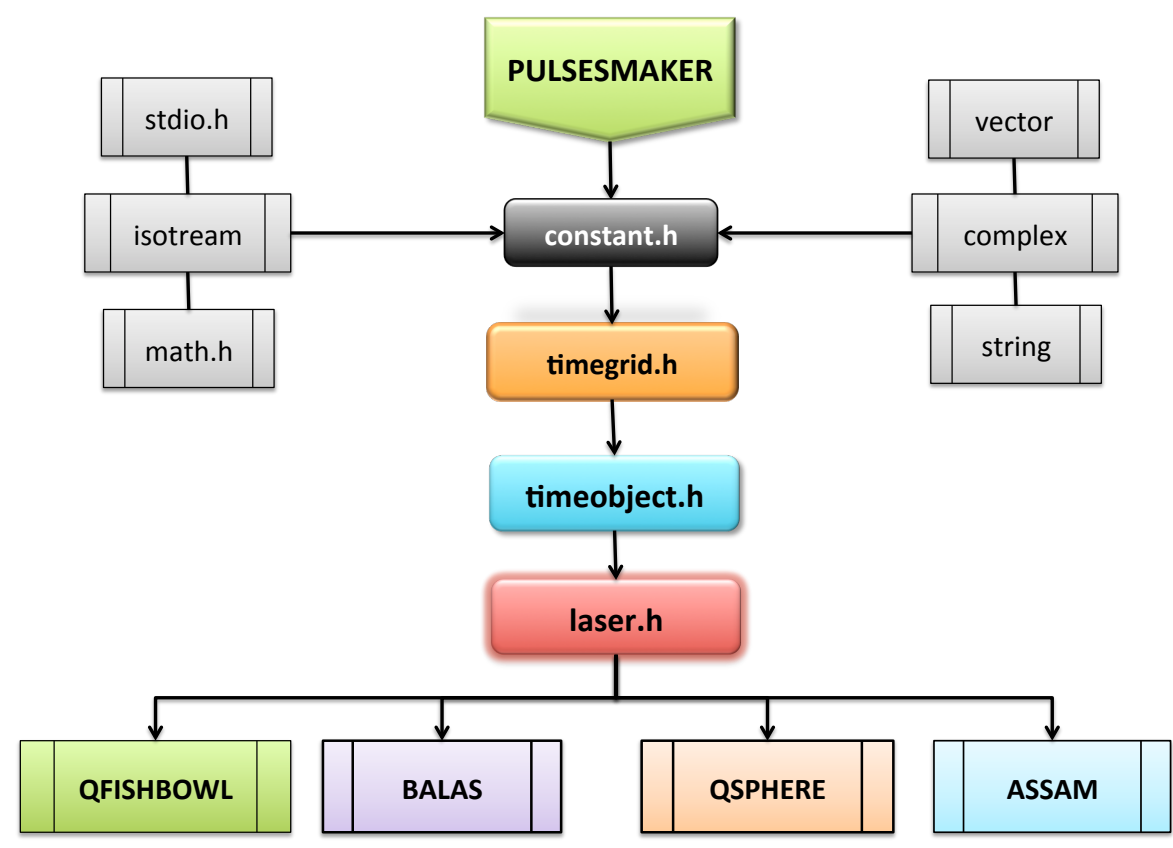

Figure 3.4: PULSESMAKER general design. A dependence diagram on the standard $\mathrm{C} / \mathrm{C}++$ libraries of the PULSESMAKER library is illustrated. The arrows define the flow direction 
BOWL, BALAS, QSPHERE, and ASSAM libraries, as a common or basic tool, to implement the laser pulses in the simulations.

\subsubsection{Time grid array}

The class timegrid.h is made of a set of member variables and member functions. The goal of this class is to construct the time and frequency axis.

The dependence diagram on the standard and others libraries is depicted in Fig. 3.5. It is shown that this class is built under a set of standard $\mathrm{C} / \mathrm{C}++$ libraries already mentioned in Fig. 3.4.

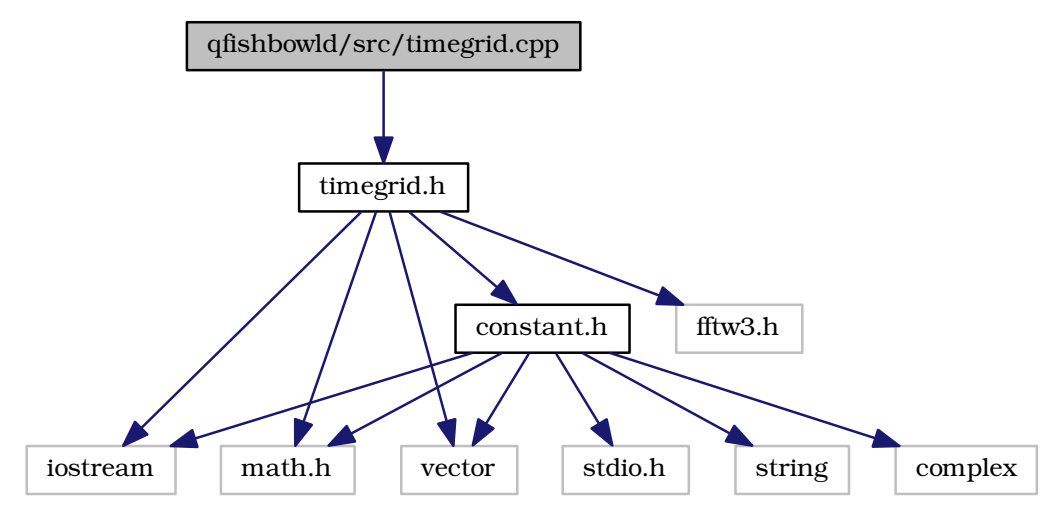

Figure 3.5: Time grid class. A dependence diagram on the Standard C/C++ libraries of the timegrid.h class is illustrated.

The time and frequency axis are the main variables of the class: $\mathbf{t}$ and $\omega$, which are defined according to Eq. (2.57)-(2.58).

Other routines to initialize the timegrid.h class can be found in detail in the Web $\operatorname{site}^{1}[120]$.

\subsubsection{Time object class}

The class timeobject.h creates a complex vector with the type defined in the FFTW library. This vector array, $\mathbf{f}$, will contain the time-domain evaluation of the electric field of the laser pulse or the vector potential.

\footnotetext{
${ }^{1}$ http://www. quantumsimlab.es/pulsesmaker/files.html
} 


\section{PULSESMAKER}

Note that timeobject.h inherits all the properties of the class timegrid.h. This means that the variables and routines used in timegrid.h can be used by the class timeobject.h.

Furthermore, the implementation of a vector FFTW allows us to compute the forward and backward FFT of $\mathbf{f}$. Then two routines to compute the forward and backward FFT are employed from the FFTW library to timeobject.h. This provides direct access to the spectrum of the electric field immediately.

In this library there are some routines to compute the vector potential by two different methods: a fourth-order Runge Kutta method and the Fourier method.

\subsubsection{Laser class}

The laser.h class is implemented with the goal to evaluate an arbitrary number of pulses $\mathrm{N}_{\text {pls }}$. The library creates a single or delayed train of pulses. Each pulse can have different characteristics, i.e., envelope, FWHM, carrier frequency, intensity and CEP. The dependence of the laser class in relation to timeobject.h, timegrid.h, fftw3.h, grid.h, are illustrated in Fig. 3.6. The logical structure of this library is based on the construction of several objects: the timegrid, and the timeobject and auxiliary variables complex vector and double variables.

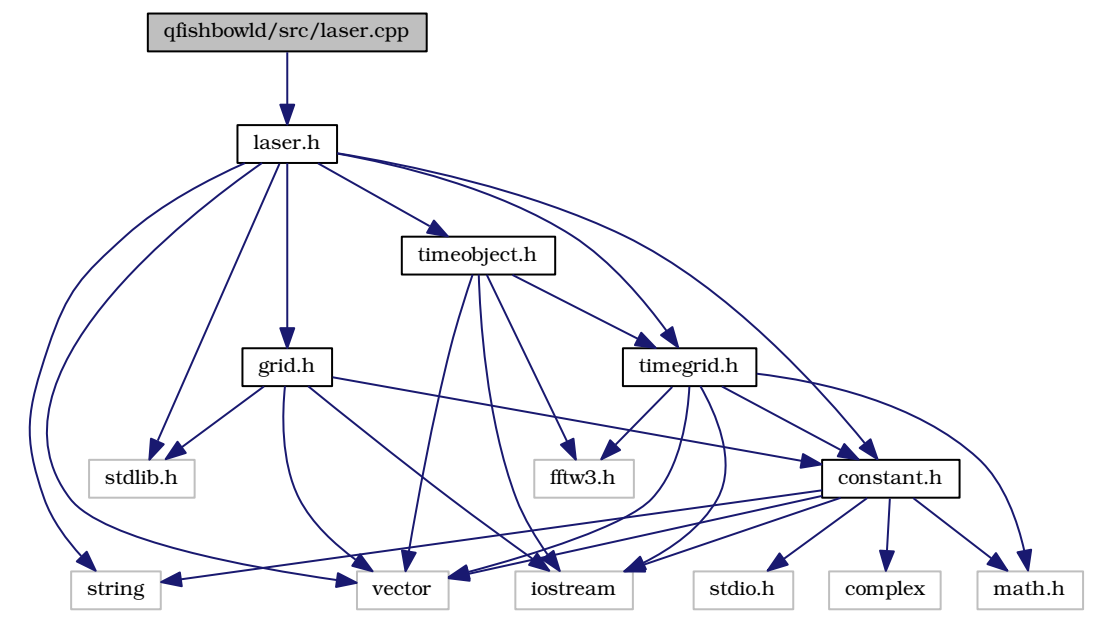

Figure 3.6: Laser class. A dependence diagram on the standard $\mathrm{C} / \mathrm{C}++$ libraries of the laser.h class is illustrated.

They will help to the implementation of two routines to compute a single or a delayed 
train of pulses. The first routine, laser $\left(\mathrm{N}_{\mathrm{pls}}\right)$, initializes the class as an object. This means that several variables, such as the peak intensity per pulse, frequency $\omega$, period $\mathrm{T}$, etc., are initialized with the size number of pulses $\mathrm{N}_{\mathrm{pls}}$. The second routine is called laser_pulses(...), and receives four parameters. These parameters are detailed in Web Site $^{1}$ [120]. The routine creates the electric field and vector potential.

PULSESMAKER has several other variables which can help at the moment to run a simulation, i.e., the time at the maximum of the envelope for each pulse and its corresponding index time, etc. Note that this library enables to choose between four different kind of pulse envelopes, namely sine-squared, gaussian, rectangular and $\mathrm{sech}^{2}$. So far, we have given a general description of how the laser pulses are built by utilizing the design strategies of software in $\mathrm{C} / \mathrm{C}++$.

The next Section will be devoted to describe the QFISHBOWL library which makes use of PULSESMAKER.

\subsection{QFISHBOWL}

The QFISHBOWL library is a large library written in $\mathrm{C} / \mathrm{C}++$ with several different classes structured within the object oriented programming paradigm. It has been designed with the main goal to integrate numerically the TDSE in cartesian coordinates by implementing the Split-Operator Spectral method described in Section 2.2.1.

The software allows the simulation of systems in $1 \mathrm{D}, 2 \mathrm{D}$ and $3 \mathrm{D}$ and it is possible to change quickly between them. The kernel of this library can be explained according to Fig. 3.7. This diagram shows the structure of classes that conform the QFISHBOWL library.

It starts with a constant class, which contains all the physical constants required to solve the TDSE. Then the grid class which is a basic ingredient in our library.

The grid.h class contains all the information about the physical region (or computational memory) where the wavefunction "lives". It is a fundamental piece for the library because the wavefunction and the hamiltonian classes are created under the grid class

Then, cartesian.h class uses all the basic elements defined on top of a grid: the wavefunction and the hamiltonian, with the aim of building specific routines to perform the time-space evolution operators.

The diagram shows finally a box with the main file or script written in $\mathrm{C} / \mathrm{C}++$ to perform the actual physical simulations by employing all the classes defined above

\footnotetext{
${ }^{1}$ http://www. quantumsimlab.es/pulsesmaker/files.html
} 


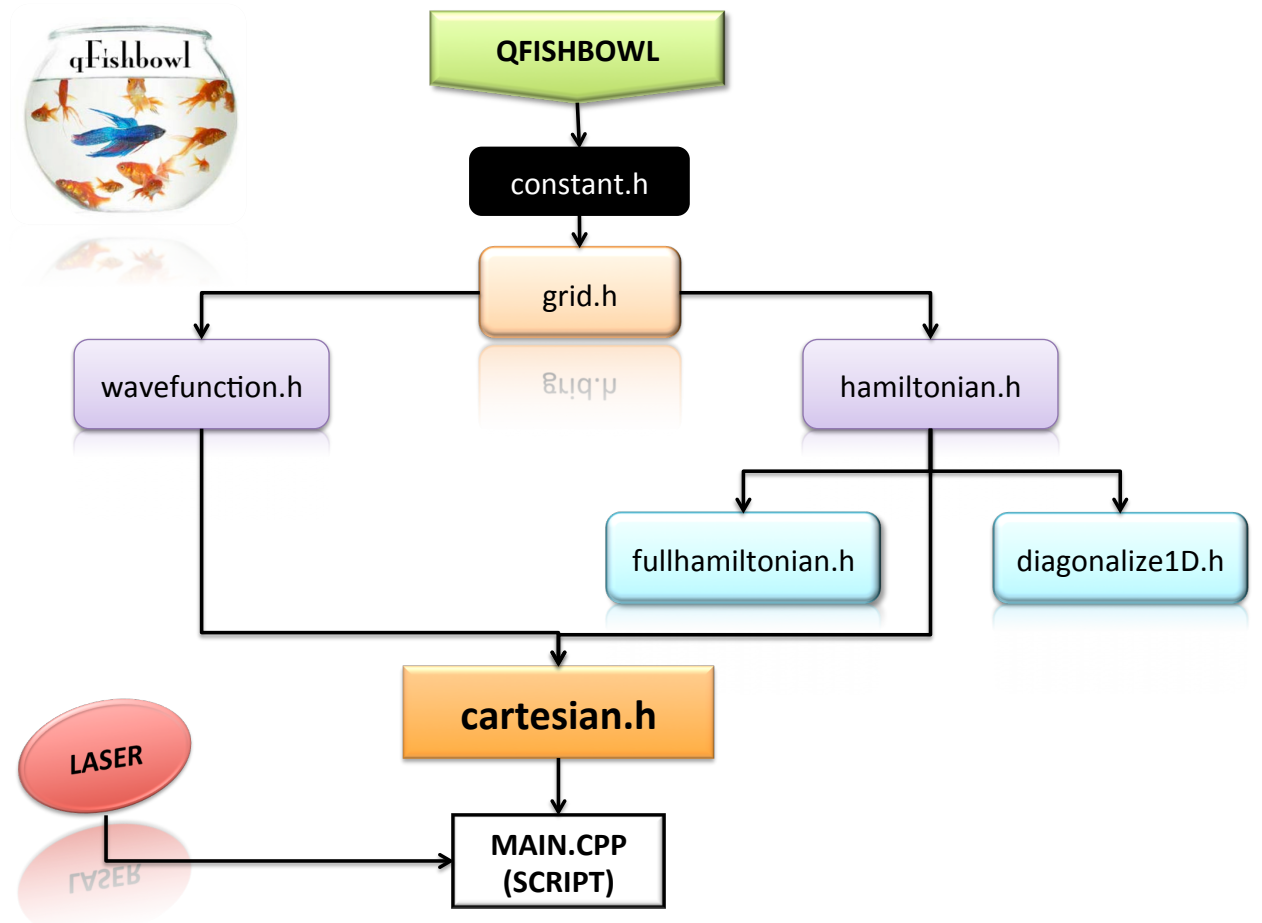

Figure 3.7: QFISHBOWL library scheme. General flow diagram of the QFISHBOWL library. The arrows denote the direction of the logical flow. 
together with the class laser.h. This class is a part of PULSESMAKER, described in Section 3.2.3.

As mentioned above the QFISHBOWL library was developed by Ruiz. This library has been extended substantially. For example, new versions of the QFISHBOWL have been developed, where the 1D Numerov Method is implemented to compute the SW, the design of two new classes, i.e., fullhamiltonian.h and diagonalize1D.h to diagonalize the Hamiltonian in 1D (as it is depicted in Fig. 3.7). These classes can be extended in a future to the $2 \mathrm{D}$ and $3 \mathrm{D}$ cases.

Another contribution to this library, is the design of dequation.h. This class computes the ionization transition amplitude according to the SFA for the interaction of laser pulses with an atomic or molecular potential, in a $p_{x}$-line and in $\left(p_{x}, p_{y}\right)$-plane.

Throughout this thesis and due to the limitation of time, we will not describe all the classes fullhamiltonian.h, diagonalize1D.h and dequation.h. However, we invite the reader to visit the reference ${ }^{1}$ [120], where more details are described about those routines. The next Sections, from 3.3.1 to 3.3.4 will be dedicated to describe the main components of the class which form QFISHBOWL: grid.h, wavefunction.h, etc.

\subsubsection{Computational grid class}

The described position-momentum coordinates in Section 2.2.1.2 of Chapter 2, are implemented in the class grid.h. This class depends on a set of standard and own libraries of $\mathrm{C} / \mathrm{C}++$ programming language. The standard $\mathrm{C} / \mathrm{C}++$ libraries are stdio.h, iostream, math.h, string, vector, etc.

Here, the own library is the class defined by constant.h, this class defines a set of constants, e.g., the $\pi$ number, the imaginary number $i=\sqrt{-1}$, the atomic unit speed of the light $c=137$ a.u., the proton mass in atomic unit $m_{p}=1836$, etc. Fig. 3.8, illustrates the required standard $\mathrm{C} / \mathrm{C}++$ libraries by the class grid.h.

Three vector arrays are defined to construct the uniform position coordinates $\mathbf{x}, \mathbf{y}$ and $\mathbf{z}$. The sizes of these computational arrays are, $N_{x}, N_{y}$ and $N_{z}$, respectively. $\mathrm{G}_{\mathbf{r}}$ is constructed by following the rules given in 2.40. Note that the position coordinates conform an uniform grid.

The momentum grid $\mathrm{G}_{\mathbf{p}}$ is also implemented in the class grid.h, in the three vector arrays $\mathbf{p}_{x}, \mathbf{p}_{y}$ and $\mathbf{p}_{z}$. The exact arrangement for the momentum components of the wavefunction $\operatorname{FFT}[\Psi(\mathbf{r})][109]$ are not the same as in $\mathrm{G}_{\mathbf{p}}\left(p_{x}, p_{y}, p_{z}\right)$. Then the order in Eq. 2.41 is changed in the momentum axis, this change is defined in grid.h. For

\footnotetext{
${ }^{1}$ http://www.quantumsimlab.es/qfishbowl/files.html
} 


\section{QFISHBOWL}

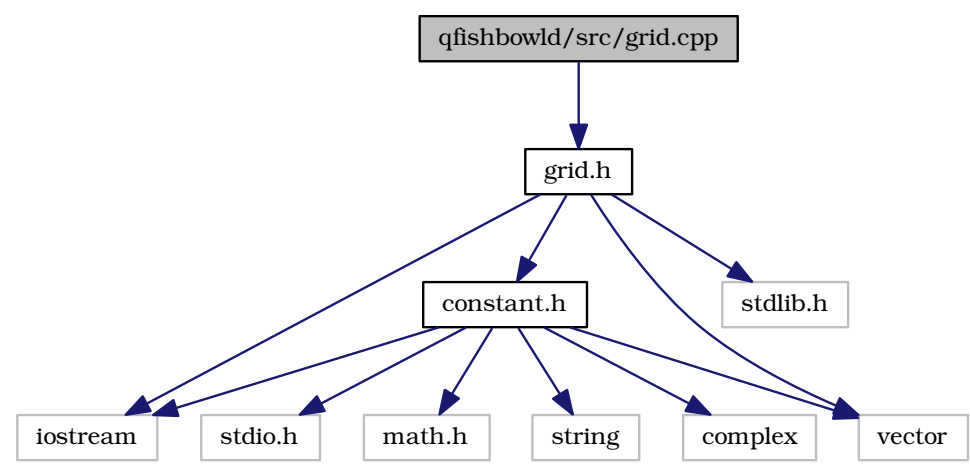

Figure 3.8: Grid class diagram. A dependence diagram on the standard $\mathrm{C} / \mathrm{C}++$ libraries of the grid.h class is illustrated.

instance, this arrangement of the momentum axis along $p_{x}$ is :

$$
\mathbf{p}_{x}=\left\{0, \Delta p_{x}, \ldots, \underline{i} \Delta p_{x}, \ldots,\left(\frac{N_{x}}{2}-1\right) \Delta p_{x}, \ldots\right\}, \text { with, } 0 \leq \underline{i}<\frac{N_{x}}{2},
$$

and,

$$
\mathbf{p}_{x}=\left\{\ldots,-\frac{N_{x}}{2} \Delta p_{x}, \ldots,\left(\underline{i}-\frac{N_{x}}{2}\right) \Delta p_{x}, \ldots,-\Delta p_{x}\right\}, \text { with, } \frac{N_{x}}{2} \leq \underline{i}<N_{x},
$$

where, $\Delta p_{x}$, is defined according to the Nyquist frequency of Eq. 2.41. A similar procedure is followed to compute the momentum axis $\mathbf{p}_{y}$ and $\mathbf{p}_{z}$.

Furthermore, it is important to point out that our implementation of the QFISHBOWL code supports the $1 \mathrm{D}$ and $2 \mathrm{D}$ numerical models. In the $1 \mathrm{D}$ case, it is easy to perform an 1D model by fixing $N_{y}=N_{z}=1$ and $\Delta y=\Delta z=1$ as well. On the other hand, the $2 \mathrm{D}$ model, i.e. the plane $(x, y)$, can be set by fixing $N_{z}=1$ and $\Delta z=1$.

Finally, some member methods or routines, i.e., set_grid $\left(N_{x}, N_{y}, N_{z}, \Delta x, \Delta y, \Delta z\right)$, are defined in this class to compute each axis, position and momentum coordinates, respectively. Another important basic routines, e.g., index $2 \mathrm{D}(a b)$, index $3 \mathrm{D}(a, b, c)$, etc., needed to transform a third-order dimensional array syntax in an one dimensional vector syntax, are also implemented here. For more detail about these routines visit $^{1}[120]$.

\footnotetext{
${ }^{1}$ http://www.quantumsimlab.es/qfishbowl/files.html
} 


\subsubsection{Wavefunction class}

The described wavefunction in Eq. (2.42) can be computationally implemented in a class of $\mathrm{C} / \mathrm{C}++$ : wavefunction.h. The dependence diagram of the wavefunction class on the standard C/C++ libraries, fftw3.h library [109] and our own grid.h class, is depicted in Fig 3.9. The dependence on the grid.h is fundamental because the wavefunction inherits all the properties of the grid class, i.e. all the variables and routines: vector arrays which contain the position and momentum coordinates, number of points and spacing grid steps, etc.

The best manipulation of the computer memory is by utilizing a first-order array or vector, and not a third-order array. This is the reason why the $3 \mathrm{D}$ wavefunction is written as a vector and not as a third-order array. A complex pointer designed to compute the forward and backward FFT is implemented in the FFTW library [109].

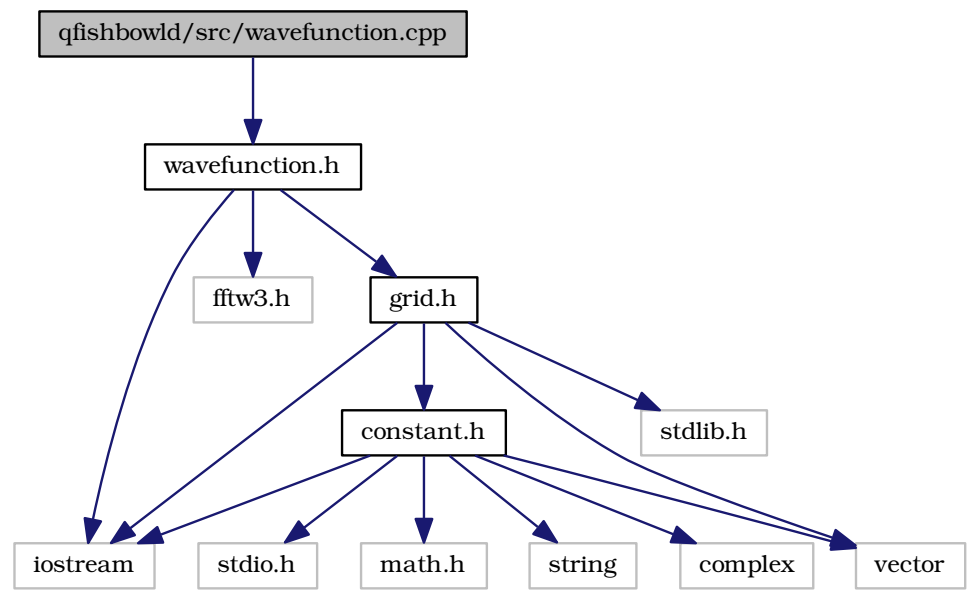

Figure 3.9: Wavefunction class. A dependence diagram on the standard $\mathrm{C} / \mathrm{C}++$ libraries of the wavefunction.h class is illustrated.

It has a particular data complex type. We have designed a pointer array of $\mathbf{w}\left[N_{T}\right]\left[N^{\prime}\right]$. Here, $N_{T}$ denotes the total number of points given by $N_{T}=N_{x} N_{y} N_{z}$ and $N^{\prime}=2$ defines the computational memory space for the real and imaginary parts of the complex wavefunction $\Psi(\mathbf{r})$. In this way this pointer contains the real part of the wavefunction in $\mathbf{w}\left[N_{T}\right][0] \equiv \mathfrak{R e}[\Psi(\mathbf{r})]$ and the imaginary part, in $\mathbf{w}\left[N_{T}\right][1] \equiv \mathfrak{I m}[\Psi(\mathbf{r})]$.

It is important to point out how the arrangement of the elements $\Psi_{\underline{k}, j, \underline{i}}$ have been carried out in the vector array $\mathbf{w}$. The first elements $\left\{\Psi_{0,0,0}, \ldots, \Psi_{0,0, i}, \ldots, \Psi_{0,0, N_{z}-1}\right\}$, 


\section{QFISHBOWL}

when $\underline{k}=\underline{j}=0$, are placed in the first $N_{x}-1$ positions of the vector array $\mathbf{w}$. The same logical process is employed to arrange the next elements when $\underline{k}=0, \underline{j}=1$, but here the corresponding elements $\left\{\Psi_{0,1,0}, \ldots, \Psi_{0,0}, \underline{i}, \ldots, \Psi_{0,1, \mathrm{~N}_{z}-1}\right\}$ are placed on the memory range of points $N_{x}$ and $2 N_{x}-1$. In the same logical way all the remaining elements of $\Psi_{\underline{k}, j, \underline{i}}$, are arranged on the computational vector array $\mathbf{w}$.

Furthermore, note that this wavefunction class contains member routines, e.g., to compute: the forward FFT and the backward FFT of the wavefunction $\mathbf{w}$, the norm of the wavefunction, the position expectation values, the momentum expectation values and the normalization of the wavefunction. This is the fundamental kernel of the wavefunction class.

\subsubsection{Hamiltonian or potential class}

The potentials of the atomic or molecular systems under study in this thesis have been implemented in a $\mathrm{C}++$ class which is named hamiltonian.h. This class is built over the position grid. $\mathrm{h}$ and contains a set of routines to evaluate Eqs. (2.43)-(2.45). Fig. 3.11, depicts the dependence on the classes, in particular on the grid.h.

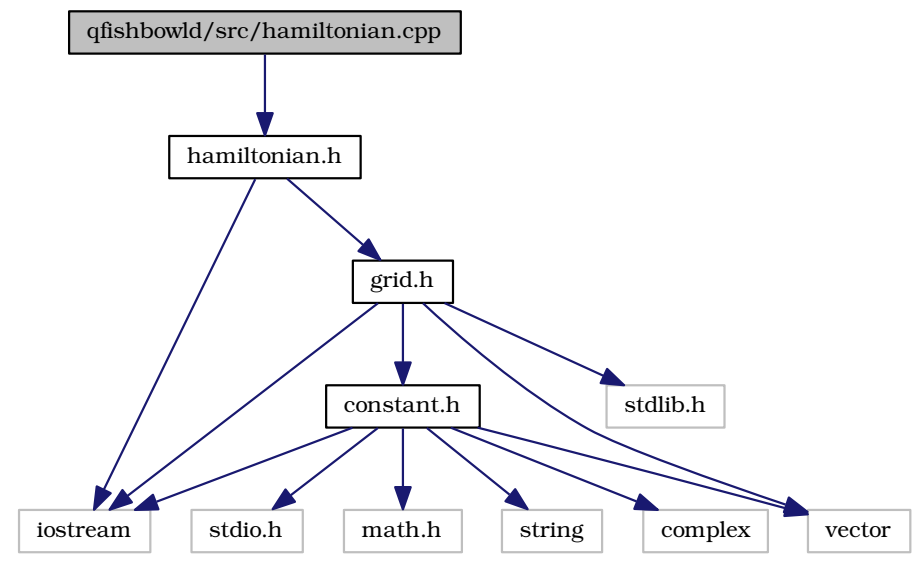

Figure 3.10: "Hamiltonian" class. A dependence diagram on the standard $\mathrm{C} / \mathrm{C}++$ libraries of the hamiltonian.h, is illustrated.

As the 3D wavefunction $\Psi(\mathbf{r})$ is computationally arranged in a pointer array $\mathbf{w}$, the potential $V_{0}(\mathbf{r})$ is implemented in a vector array $\mathbf{v}$ as well. The vector array dimension is defined by the total number of points $N_{\mathrm{T}}$. In addition, this vector will contain all 
the elements $V_{0, \underline{k}, j, \underline{i}}=V_{0}\left(z_{\underline{k}}, y_{\underline{j}}, x_{\underline{i}}\right)$.

The Hamiltonian class also has some routines to evaluate the different potentials, e.g., set_v_hlike3D(...), set_v_hlike2D(...), set_v_hlike1D(..), which compute the hydrogen-like soft core potential well. This hamiltonian.h class also evaluates the potential energy given by Eqs. (2.43)-(2.45).

\subsubsection{Evolution operators: cartesian class}

Numerically we implement the spectral split operator method at each time $t_{n}$, by taking advantage of the FFTW3 library [109]. In particular, we have developed a class named cartesian.h. This class makes use of all the other classes grid.h, wavefunction.h and hamiltonian.h, as it is depicted in Fig. 3.11. The aim of this cartesian class consists of implementing the propagator operators related the the split-operator spectral method.

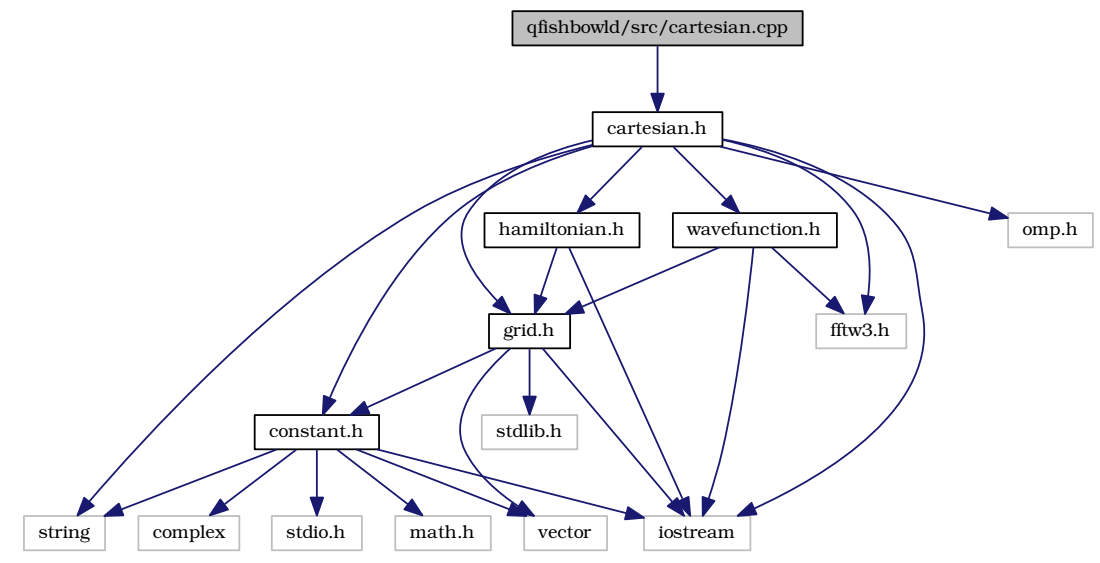

Figure 3.11: Cartesian class. A dependence diagram on the standard $\mathrm{C} / \mathrm{C}++$ libraries of the cartesian.h class, is illustrated.

Several routines have been implemented in the cartesian class to compute some observables, such as the momentum distribution, the expectation kinetic and potential energy values, the left-right asymmetry, the routines to compute the scattering waves, and the projection of the evolved wavefunction on the scattering waves, etc.

Other important routines to compute the evolution of the wavefunction, namely those pointed out in (i), (ii) and (iii) of Eq. (2.35), are included in the cartesian class.

These routines, prop_kinetic $(\ldots, \Delta t)$ and prop_potential $(\ldots, \Delta t)$, compute the 


\section{QFISHBOWL}

action of the kinetic and potential evolution operator $(i)$ and $(i i)$, (without taking into account the laser pulse), respectively.

The routine to evaluate the kinetic operators $(i)$ and $(i i)$, prop_kinetic $(\ldots, \Delta t)$, utilizes the parallel version in OpenMP of the forward and backward FFT. It is important to point out that the algorithm for the FFT implemented in [109] has a number of operations $O(\mathrm{~N} \log \mathrm{N})$. This means that the computation of the FFT allows us to obtain faster numerical results than any other algorithm $O\left(\mathrm{~N}^{2}\right)$, such as the Discrete Fourier Transform.

The terms (i) and (iii) in Eq. (2.35) are calculated by a single routine which is named prop_kinetic $(\ldots, \Delta t)$. This routine makes a FFT of the wavefunction $\Psi(\mathbf{r})$. Then, by employing the kinetic evolution phase $\exp \left(-i \frac{\mathbf{p}^{2}}{2} \Delta t\right)$ on the momentum spectral representation $\Psi(\mathbf{p})$ of the wavefunction, we evaluate Eq. (2.36).

It is worth of noting that the phase factor in the momentum space has this form, $\exp \left(-i \frac{\mathbf{p}^{2}}{2} \Delta t\right)$, when the length gauge is utilized to describe the problem. In the velocity gauge, this phase factor becomes, $\exp \left[-i \frac{1}{2}\left(\mathbf{p}+\mathbf{A}\left(t_{n}^{\prime}\right)\right)^{2} \Delta t\right]$, with $t_{n}^{\prime}=t_{0}+\left(n+\frac{1}{2}\right) \Delta t$. The denoted terms ( $i$ ) and (iii) can be evaluated by replacing $\Delta t \rightarrow \Delta t / 2$ in the routine prop_kinetic(.., $\Delta t)$ (for instance, see Eq. (2.35)).

The term (ii) in Eq. (2.35) is computed in the position space by evaluating numerically the phase factor, $\exp \left(-i V_{\underline{i}, \underline{j}, \underline{k}} \Delta t\right)$, times the wavefunction. This is implemented in the routine prop_potential $(\ldots, \Delta t)$. This phase factor also will depend on which gauge is used to study the problem. We have implemented and employed both the length and velocity gauges.

In particular, Fig. 3.12 depicts a snapshot of a portion of the code. It shows the logic of the time evolution of the wavefunction under the action of a laser pulse. The depicted propagation scheme is equivalent to the one defined by Eq. (2.35).

The QFISHBOWL library will be utilized to perform some of the calculations regarding the physics problems addressed in Chapter 4 and 5 . 


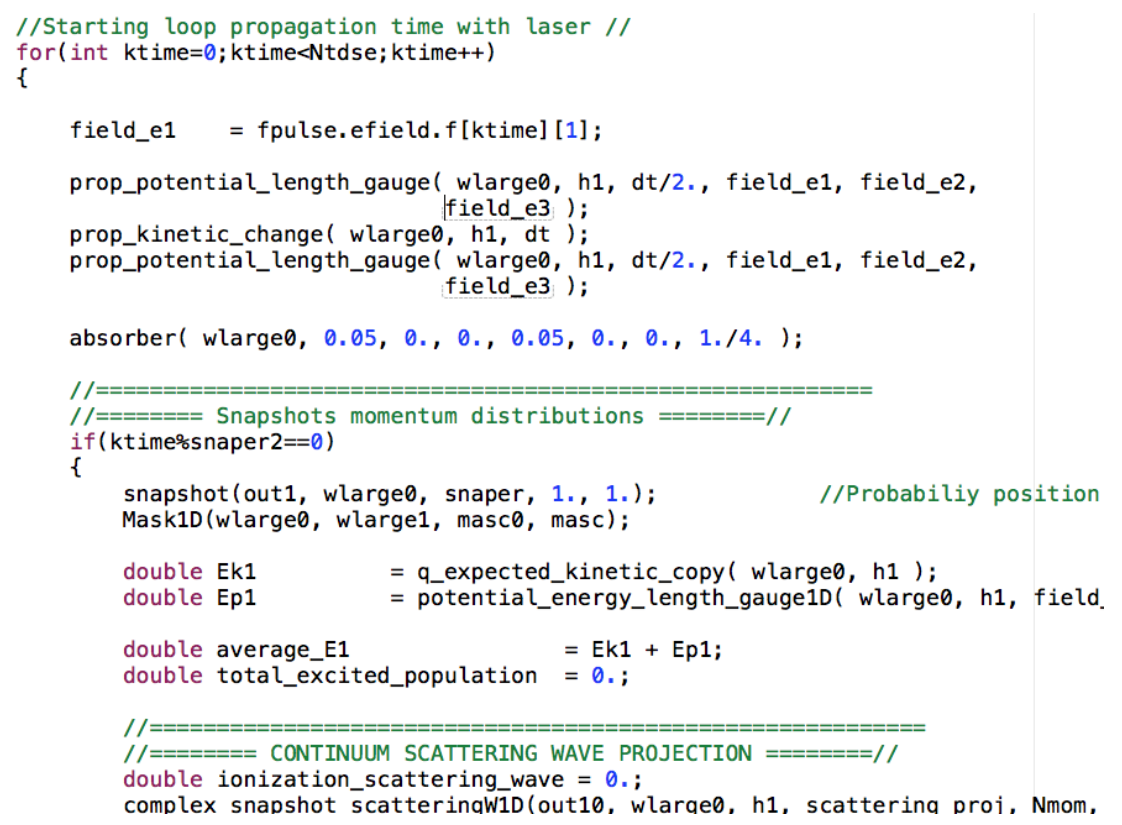

Figure 3.12: Example code: QFISHBOWL. Example of a code where the routines related to the time-evolution propagators are employed for the evolution of the ground wavefunction which interacts with a laser field. The evolution operators, prop_potential_length_gauge(...) and prop_kinetic_change (...) are new routines to compute the time evolution of the wavefunction. 


\subsection{BALAS}

The implementation of the numerical solution of Eq. (2.70) can be performed by using the split-operator spectral method joint with the Crank-Nicolson method. Both methods have been computationally implemented in a library written in $\mathrm{C} / \mathrm{C}++$. We have termed this library BALAS [113].

Here we will give a brief review of the BALAS library implemented to solve the TDSE in cylindrical coordinates. The architecture is explained in the Fig. 3.13. Similar to the QFISHBOWL, BALAS requires a constant class, tool.h, etc. It is worth to mention that BALAS has been developed to compute the TDSE under both numerical schemes: a nonuniform grid scheme, and an uniform grid scheme. This means that the split-operator spectral method is implemented in the first one, and in the second one, the Crank-Nicolson technique.

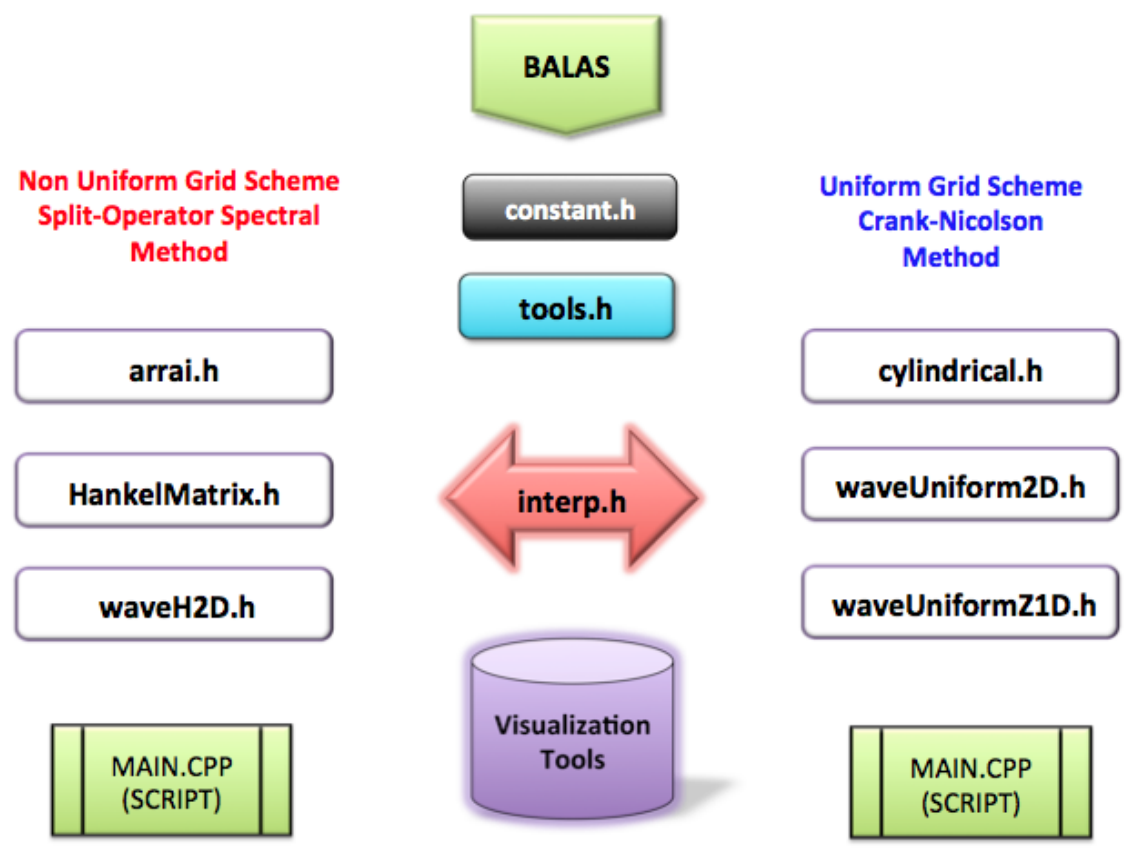

Figure 3.13: BALAS library scheme. This picture depicts the classes which form the BALAS library. Two propagation or evolution schemes of the wavefunction have been developed: (i) non-uniform grid and (ii) uniform grid. The first implements the split-operator spectral method. The Crank-Nicolson numerical technique is implemented in the second scheme. Both of them are connected by an interpolating class which is needed to transform the wavefunction from the uniform grid to the nonuniform one and vice versa. Furthermore, visualization tools based on VisIt software [121] were also recently implemented by the author of this thesis. 
The spectral split operator method requires to compute a Hankel transform along a cylinder of radius $\rho$ and a FFT on its cylinder $z$-axis. From a computational point of view, the Hankel transform is not so efficient as it is the FFT. The HT has an operation number around $O\left(N^{2}\right)$ [114]. On the other hand, the Crank-Nicolson method only requires a computational operation number about $O(N)$ [103] along the radial coordinate and the same along the $z$-direction. This means that in case of cylindrical coordinates the split-operator method will be slower compared to the Crank-Nicolson. For this reason, we use the Crank-Nicolson method to address our numerical solution of the TDSE.

In spite of the fact that the split-operator spectral method is implemented in the BALAS library [113], it is not used through this thesis to handle any physical problem. The main reason for that is the large total number of operations needed compared to those required by the Crank-Nicolson method.

\section{Hankel Transform}

In addition, note that through this thesis the time-evolution of the wavefunction $\Psi(\rho, z, t)$ is performed on an uniform position-space grid $(\rho, z)$ (see, Fig. 3.13). Nevertheless, when we compute the momentum distribution of the continuum wavefunction part $\Psi_{c}(\rho, z, t)$, e.g. projecting on PW, a FFT will be employed along $z$-axis, but throughout $\rho$-axis a HT is required.

The uniform grid position-space defined by Eq. (2.85) is implemented in a general class in $\mathrm{C} / \mathrm{C}++$ which is termed waveUniform2D.h. The nonuniform radial $\rho^{\prime}$ grid Eq. (2.86) is defined in a set arrai.h and HankelMatrix.h. These classes contain all the needed routines to compute the Fast Hankel Transform which is described in the reference [114]. It is also implemented another class, interp.h, to perform the interpolation of the wavefunction from the uniform to the nonuniform radial grid as it is depicted in Fig. 3.13. Note, that this interpolation is done along the $\rho$-axis.

Furthermore, the BALAS classes have been implemented under the architecture of the MKL Intel library [115]. We take advantage of the MKL optimization routines to compute the interpolation of a function, the FFT, the matrix product and the MKL variable types. These routines allow us to perform our calculations utilizing the Intel processors with the maximum possible optimization for each employed routine [115].

The axis described in Eq. (2.87) is implemented in the HankelMatrix.h class [113]. We have already prepared the required position-space and momentum-space grids to address the problems under the cylindrical symmetry which will be studied throughout this thesis. For more details about the documentation of the classes regarding the 
BALAS library, visit the Web Site ${ }^{1}[120]$.

BALAS library is utilized to carry out some of the calculations related to the physical problem addressed in Chapter 5. In particular, to simulate the attosecond streak camera under the conditions of an asymmetric molecular potential.

\subsection{QSPHERE}

One of the interesting physical problems is to model the ATI process driven by linearly and circularly polarized laser fields or simply the study of the photoelectron momentum distributions. To perform a computational calculation in a 3D cartesian coordinates, a large number of points and also operations is required.

QFISHBOWL library and BALAS have been well organized. However, the main disadvantage is given by the larger number of points needed to simulate a full 3D calculation. These libraries only can be used in larger clusters.

For this reason, a new library in spherical coordinates, QSPHERE (Quantum simulations in the SPHERical coordinatEs) [119], was developed.

The aim of this new code is to reduce the large number of grid points by changing the solution of the TDSE from the cartesian coordinates to the spherical coordinates. The advantage of this new coordinate system consists of the expansion of the wavefunction on Spherical Harmonics which deal with the polar and azimuthal coordinates. Few points will be required to evaluate the evolution of the wavefunction along the polar and azimuthal coordinates [122, 123, 124].

The main algorithm consists of evaluating the split-spectral reduced radial operator for the wave equation [122] together with the reduced wavefunction in two different schemes. The reduced radial kinetic operator (see Eqs. (1)-(2) in reference [122]), can be evaluated by a Fourier transform based on the sine function, i.e., the $\sin \left(k_{r} r\right)$. This means that the corresponding evolution operator to the radial coordinate can be evaluated by a Sine Transform or FFT. Here we have tested both methods, and for efficiency we chose the Sine Transform. We have taken advantage of the Fast Trigonometric Transform (FTT) of the MKL Intel library [115], to compute the forward and backward sine transform of the reduced radial wavefunction. This routine takes advantage of the OpemMP parallelization paradigm of the FTT implemented in such MKL.

The angular part of the Laplacian Operator is evaluated by doing a Spherical Harmonic Transform [125]. By computing a similar phase factor corresponding to the

\footnotetext{
${ }^{1}$ http://www . quantumsimlab.es/balas/files.html
} 
Eq. (4) of reference [122] the angular part of the Laplacian is computed. In particular, QSPHERE is right now implemented to the case where the laser pulse is linearly polarized along $z$-axis. Thus, we can neglect the dependence of the wavefunction on the azimuthal coordinates. It is so, because the linearly polarized laser excites only states of magnetic momentum $m=0$. In such a case, only a Legendre Transform is required to evaluate the angular part of the Laplacian Operator.

The architecture of QSPHERE can be summarized in Fig. 3.14. The logical flow of the classes is the following, the constant.h class has some constants, grid.h is a class which builds the radial and polar coordinates. This class is different from grid.h of QFISHBOWL. QFISHBOWL has used a vector array by using the standard $\mathrm{C}++$ vector library. However, in QSPHERE the arrays which will contain the axis coordinates are defined as double pointers.

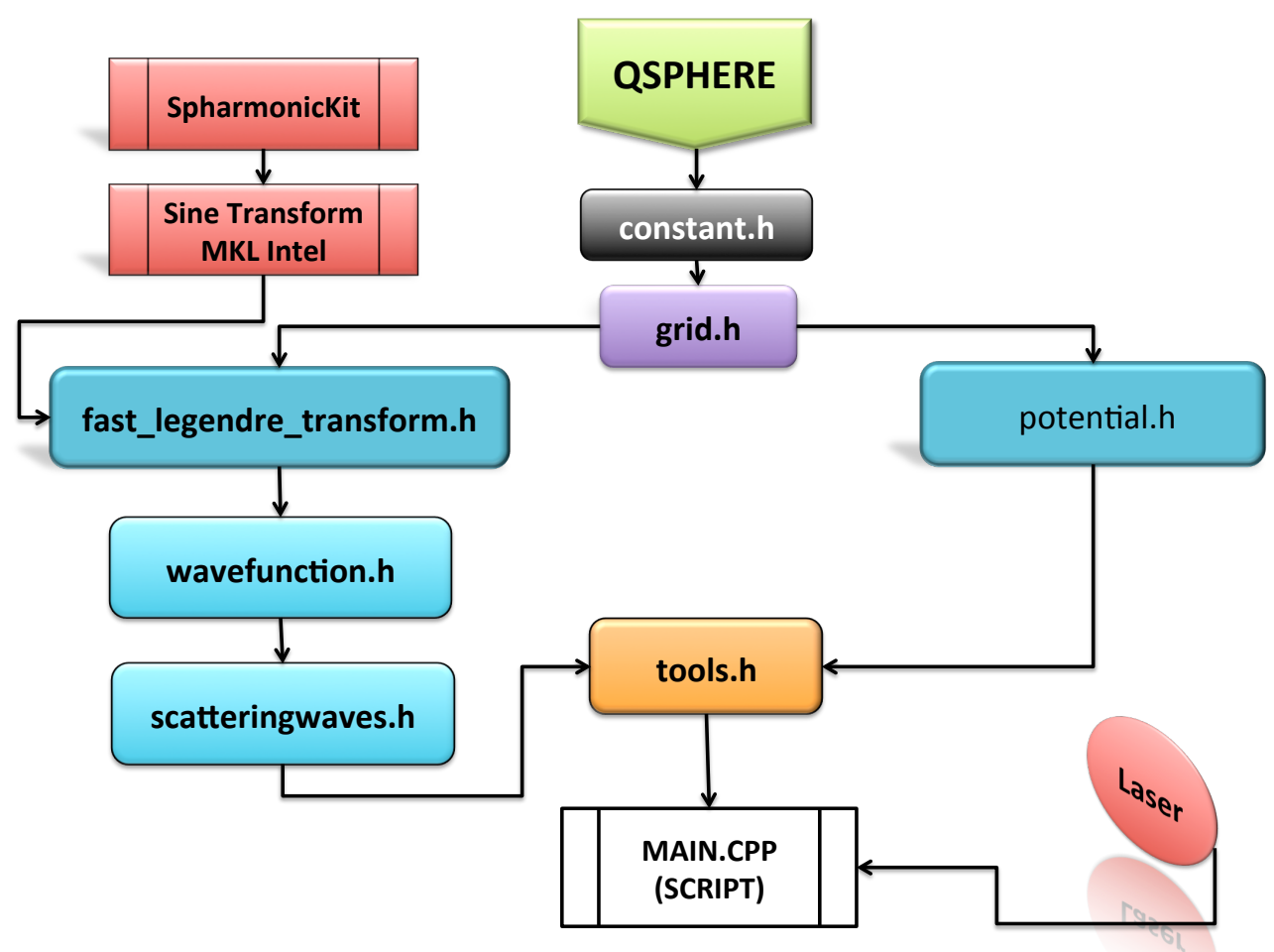

Figure 3.14: QSPHERE library scheme. This picture depicts the classes which form the QSPHERE library and its main dependences: SPHARMONICKIT [125] library and MKL Intel Fast Sine Transform [115]. Furthermore, visualization tools based on the VisIt software [121] are also implemented recently by the author of this thesis. The arrows denote the direction of the logical low.

This is quite advantageous, because we are controlling the memory of the computer 
by ourselves and not by some predefined library. This pointer manipulation is a little bit more efficient than the predefined vector standard $\mathrm{C}++$ library. The classes fast_legendre_transform.h, potential.h, wavefunction.h, scatteringwaves.h and tools.h form the so-called QSPHERE library and the reader can find more details in the Web Site ${ }^{1}[120]$.

Then, in the same way as the QFISHBOWL library application, QSPHERE can simulate a physical problem by implementing a main.cpp program that has a scriptlike structure, similar to the MATLAB script. For instance it is possible to write a script to compute a FFT or a spline of a function. This code has been used to carry out the simulation presented in the submitted paper: High harmonic generation enhanced by coherent population return.

Finally, by making use of the described theoretical tools in Chapter 2, and in the current Chapter 3, we will study how the main questions regarding the extraction of structural information about the target and electron dynamics of the photoemission processes are addressed throughout the Chapters 4, and 5, respectively.

\footnotetext{
${ }^{1}$ http://www .quantumsimlab.es/qsphere/files.html
} 


\section{Amplitude and phase retrieval techniques: theoretical viewpoint}

\subsection{Introduction}

The advent of new XUV sources [126, 127] and in particular the attosecond pulses [3] has opened new opportunities for imaging atomic and molecular systems with unprecedented temporal and spatial resolution [17, 18]. Attosecond pulses have a broadband spectrum in the XUV range and can ionize an atom or a molecule by a single-photon absorption leading to the emission of an EWP. This EWP contains information about both the attosecond pulse $[62,128]$ and the underlying atomic or molecular system $[17,66]$. The spectrum of the attosecond pulses and the bound-free dipole transition matrix element are mapped onto the amplitude and phase of the photoelectron spectrum.

The dipole transition matrix element contains structural information [5, 65] about the initial state. Ionization by attosecond pulses and measurements of the electron spectra provide then access to the absolute value of the dipole transition matrix element over a broad range of energies, but the phase of this quantity remains largely unknown and difficult to measure. For a complete characterization of the system, the knowledge of the electron phase is essential. It provides structural information, but also insight into the ionization dynamics, i.e., the Wigner time delay.

In this Chapter, we will propose two general techniques for the complete characterization of the EWP and the complex dipole transition matrix element. In contrast to previous works $[58,61,62]$ we do not focus on the characterization of the attosecond pulse itself but rather on the characterization of the dipole transition matrix element. 


\section{INTRODUCTION}

We build upon the previous demonstration of the measurement of the EWP phase difference [66] to propose a general interferometric method for full characterization of the complex EWP and the dipole transition matrix element.

As a first scheme, we introduce the Quantum Spectral Phase Interferometry for Direct Electron-wavepacket Reconstruction (QSPIDER) technique [67]. This method is a variant of the SPIDER $[20,21]$ approach, but here it is applied to quantum wavefunctions.

The SPIDER technique is an interferometric measurement of the electric field in short laser pulses. It uses a non-recursive algorithm [129] to extract the phase differences between the different frequency components of the pulse.

The SPIDER technique can be employed to characterize attosecond pulses as well. As was demonstrated by Quere and co-workers [58, 62, 61], the spectral phase of such pulses can be obtained by applying the SPIDER approach to electron wavepackets generated by photoionization of an atom by the attosecond pulses, assuming a constant transition dipole. A spectral shear is then introduced by the presence of a moderately strong IR field. The observable that contains the interferogram is the photoelectron momentum distribution.

We demonstrate that this technique [62] can be extended to characterize the amplitude and phase of the dipole transition matrix element of the ionization step.

In the second technique, we extend QSPIDER to a bi-dimensional momentum plane in analogy to the optical lateral shearing interferometry method [130, 131]. This optical method reconstructs the spatial wavefront of the laser beam by a simple interferometry scheme [130]. In this method two copies is created and then the spatial shearing are introduced in the two perpendicular directions $[132,133,134]$. From the resulting two interferograms the two partial derivatives along the perpendicular directions, i.e., $\frac{\partial \phi}{\partial x}$ and $\frac{\partial \phi}{\partial y}$, are recovered. Thus, by integrating such a phase gradient, the wavefront of the laser can be recovered. Similar steps can be employed with the electrons.

The organization of this Chapter is as follows. We define two main Sections: the first one 4.2 will be devoted to study how the complex EWP and its associated dipole transition matrix element can be reconstructed by making an analogy to the optical SPIDER technique. We start this Section reviewing the main SPIDER elements. We describe how these elements can be used with matter waves. We set up the concept of an EWP in the Section 4.2.1. The next Section 4.2.2 will be focused to show how two EWP copies can be launched to the continuum by the subsequent application of an APT of two pulses. In Section 4.2.3, we show how an ultrashort IR laser pulse introduces a relative spectral or momentum shearing into the EWP. To this end, we 
use the SFA formalism. This is formally equivalent to the spectral shearing introduced in one of the laser beam copies. Then, we will study the ionization mediated by the APT in presence of the moderated IR streak laser in Section 4.2.4. The resulting momentum streaked interferogram will contain full information about the EWP phase difference. Section 4.2 .5 will be dedicated to study how the IR induces an extra phase in the case of long-range potentials. We describe the QSPIDER implementation in Section 4.2.6. Then, we will be able to apply the same SPIDER algorithm to extract the EWP phase and the dipole phase. The test systems and the results will be addressed in Section 4.2.7 by employing the SFA. Furthermore, we present in Section 4.2.8 the results of QSPIDER under a reduced 1D-TDSE model.

The second Section 4.3 will be dedicated to extend our 1D approach to characterize the EWP on a bi-dimensional momentum plane. In analogy to the optical lateral shearing technique, we will construct two perpendicular lateral momentumshearing interferograms to reconstruct the EWP phase or the dipole phase. Then, in Section 4.3.1 we formally introduce the concept of lateral momentum-shearing by employing the SFA. In addition, we describe the new steps needed for the QSPIDER lateral momentum-shearing technique in Section 4.3.2. In the next Section 4.3.3 we employ all the methodology to extract the complex EWP and complex dipole by using the lateral momentum-shearing technique. We use two general descriptions to compute the interferograms, namely the SFA and the full 2D-TDSE. Finally, we present our conclusions in Section 4.4.

\subsection{Quantum spectral phase interferometry for direct EWP reconstruction}

In this Section we will give a brief description of the optical SPIDER technique in order to set its main characteristics and procedures. In addition, we indicate a general way to the EWP characterization by using the novel available attosecond science tools.

One of the optical techniques to characterize the electric field in an ultrashort pulse is the so-called SPIDER. This is used to retrieve the spectral amplitude and phase of the electric field of an ultrashort IR laser pulse.

Let us assume that a single unknown ultrashort pulse with electric field $E(t)$ enters to a spectrometer. Thus, a broad spectrum $S(\omega) \propto|E(\omega)|^{2}$ will be measured (see Fig. 4.1a)). We denote the electric field $E(\omega)$ on the spectral domain as $E(\omega)=$ $\int_{-\infty}^{+\infty} d t E(t) e^{i \omega t}$. But in the detection process of the intensity in the spectral domain we loss the phase. 
Interferometric techniques have been successfully employed to reconstruct information about the spectral phase of a laser pulse [20,21]. SPIDER is a special kind of these interferometric techniques applied to retrieve the spectral phase for an ultrafast electric laser field. According to Anderson et al. [21], the basic concept of SPIDER can be explained by two general steps.

First, the unknown electric field $E(t)$ is split into two identical copies $E^{(1)}(t)=$ $E^{(2)}(t)$. Then, these copies are delayed one from the other by an amount $\tau$ as it is pointed out in the left plot of Fig. 4.1b). If these two copies enter to a spectrometer, then we will measure an spectral interferogram pattern $S(\omega)=\left|E^{(1)}(\omega)+E^{(2)}(\omega) e^{-i \omega \tau}\right|^{2}$. This interferogram is depicted in the right plot of Fig. 4.1b). Mathematically, this spectral interferogram losses the original phase of the electric field $E(t)$. Thus, the fringes are only related with the delay $\tau$ between the two laser pulses copies.

Second, a relative frequency shearing $\Delta \omega$ or shift is introduced into one of the delayed copies of the pulse. In our scheme depicted in Fig. 4.1c), the relative shearing is introduced on the second copy $E^{(2)}$ of the original electric field. The measured interferogram in SPIDER can then be written as $\mathrm{S}(\omega)=\left|E^{(1)}(\omega)+E^{(2)}(\omega+\Delta \omega) e^{-i \omega \tau}\right|^{2}$ [20]. A small spectral shift is found between the no-sheared interferogram in Fig. 4.1b) and the sheared one in Fig. 4.1c). This shift can be observed by the black vertical line. This SPIDER interferogram now contains full information about the phase difference between both laser copies.

SPIDER uses a non-recursive algorithm [129] to extract the phase differences between the different frequency components of the pulse. The spectral phase difference $\phi(\omega+\Delta \omega)-\phi(\omega)$ between the two signals is encoded in the position of the fringes in the interferogram. It can be retrieved using a four-step algorithm which consists of a FFT to a pseudo-temporal domain, a filtering operation to isolate the AC component, a removal of the extra phase introduced by the delay $\tau$ and finally an inverse FFT [129]. If the shear $\Delta \omega$ is small, the obtained phase difference approximately yields the derivative of the spectral phase $[129,135,136]$.

On the other hand, it is well known that when a "long pulse", i.e., picosecond or femtosecond, in the XUV range $[137,138]$ interacts with an atom, the bound electron absorbs a XUV photon promoting it to a continuum state. The ionized photoelectron energy has a very short spectral energy bandwidth and therefore a very well defined central energy [138]. This process is very good explained by perturbative theory which is the basis of the Einstein's photoelectric effect.

The attosecond science allows us the study of such a process in a broad frequency range $[66,17]$. When a short attosecond pulse interacts with an atom or molecule 

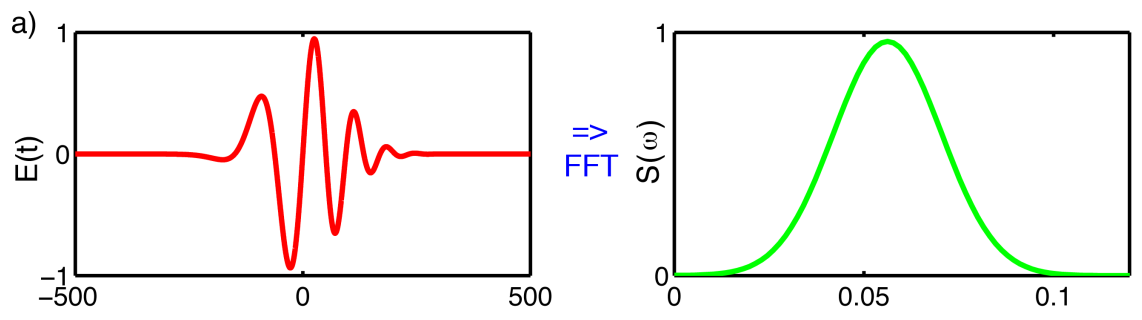

b)
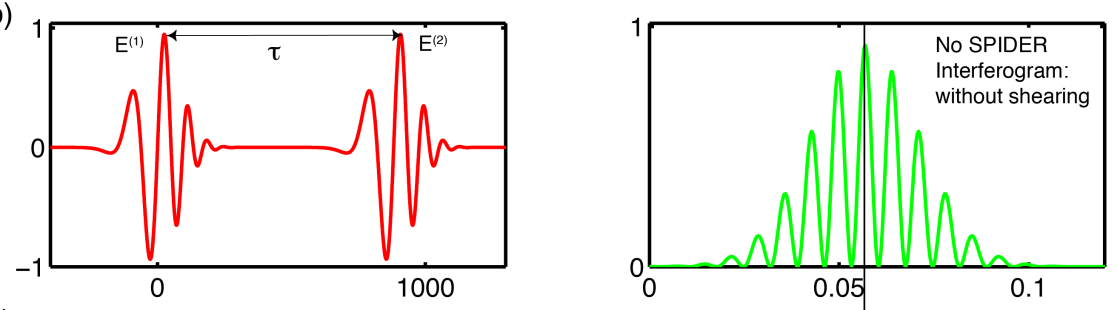

c)
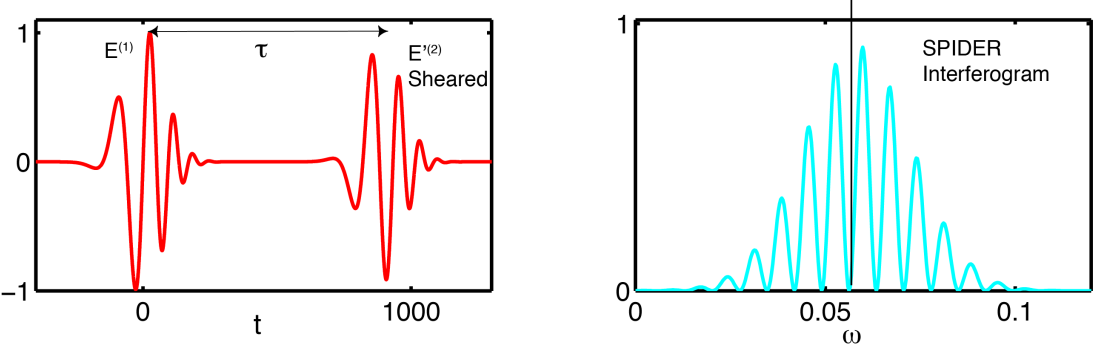

Figure 4.1: Optical SPIDER scheme. The row a) depicts the electric field oscillations $E(t)$ of a laser pulse as a function of time (left plot) and its intensity spectrum signal (right plot). b) The two laser copies $E^{(1)}(t)$ and $E^{(2)}(t)$ which are delayed by $\tau$ (left plot) and the corresponding interferogram pattern. c) The same as in b) but here the second pulse $E^{\prime}(2)(\omega+\Delta \omega)$ is relative sheared $\Delta \omega$ with respect to the first one $E^{(1)}(\omega)$ (left). We also include the SPIDER interferogram which contains full information about the phase difference between both copies of the original pulse $E(t)$. The black vertical line shows the shift between the maximum of the interferogram without shearing and the SPIDER interferogram itself. 
the same Einstein's effect occurs. However, due to the broad frequency spectrum of an attosecond pulse, the photoelectrons are emitted into the continuum have a broad electron bandwidth energy as well.

Our motivation is to study if we can construct a good analogy of the SPIDER technique to the EWP. To this end, we will take use of the attosecond science tools and mainly the attosecond streak camera concept [3]. As we will demonstrate by the subsequent application of two attosecond pulses delayed on time, two delayed EWPs are launched onto the continuum. Due to the same central frequency of both XUV pulses, the two EWPs will interfere in the momentum space. However, such an ionized signal does not contain information about the phase difference of the EWPs or the dipole transition matrix element. Similar to SPIDER, we will need to introduce a relative shearing between the delayed EWP copies. The attosecond streak effect can be employed to this end [67].

The next Sections will be devoted to study how an EWP is launched onto the continuum by a SAP. According to the first order perturbative theory, we will show that this EWP encodes also information about the bound-free dipole transition matrix element.

\subsubsection{Photoionization by a single attosecond pulse}

The quantum treatment of the electron photoionization by a single XUV attosecond pulse can be done by the first-order perturbation theory [139] and the full numerical integration of the TDSE. Here we use both theoretical tools to show that an EWP carries information about the XUV attosecond pulse itself, i.e., its spectral bandwidth, and the structure of the system, i.e., bound-free the dipole transition matrix element. We will see that different structural information about the dipole phase is obtained if the perturbative theory ${ }^{1}$ or the TDSE is used to describe the emitted EWP by the XUV radiation.

According to first-order perturbation theory [86], the bound-free complex transition amplitude $a(\mathbf{p})$ of the emitted EWP by the XUV attosecond pulse $\mathbf{E}_{\mathrm{X}}(t)$ from a bound state $\left|\Psi_{0}\right\rangle$, at the initial time $t_{0}$, to the continuum state $|\mathbf{p}\rangle$ of final asymptotic momentum $^{2}$, at the final time $t_{f}$, reads:

\footnotetext{
${ }^{1}$ It is in case that the final continuum state is considered a plane wave.

${ }^{2}$ Note the asymptotic momentum $\mathbf{p}$ is the same as $\mathbf{k}$ vector wave (in atomic units). For instance, see scattering waves in Section 2.2.1.4.
} 


$$
a(\mathbf{p})=i \int_{t_{0}}^{t_{f}} d t^{\prime} \mathbf{d}(\mathbf{p}) \cdot \mathbf{E}_{\mathrm{X}}\left(t^{\prime}\right) e^{i\left[\frac{1}{2} \mathbf{p}^{2}\left(t^{\prime}-t_{f}\right)+I_{p}\left(t^{\prime}-t_{0}\right)\right]} .
$$

where $I_{p}$ is the ionization potential of the initial bound state and $\mathbf{d}(\mathbf{p})=-\left\langle\mathbf{p}|\mathbf{r}| \Psi_{0}\right\rangle$ is the complex dipole transition matrix element bound-free for the atom or molecule. The electric field of the attosecond XUV source is $\mathbf{E}_{\mathrm{X}}(t)=E_{0, \mathrm{X}}(t) \operatorname{Re}\left[e^{-i \omega_{\mathrm{X}} t+i \varphi_{\mathrm{X}}(t)}\right] \mathbf{e}_{z}$, where $E_{0, \mathrm{X}}(t)$ is the pulse envelope, $\omega_{\mathrm{X}}$ its carrier or central frequency and $\varphi_{\mathrm{X}}(t)$ the time dependent phase of the pulse. Note that the XUV attosecond pulse $\mathbf{E}_{\mathrm{X}}(t)$ is linearly polarized along the $z$-axis.

For this attosecond XUV pulse definition, the complex EWP can be written:

$$
\begin{aligned}
a(\mathbf{p})= & A(\mathbf{p}) e^{i \varphi(\mathbf{p})} \\
A(\mathbf{p})= & \frac{1}{2}\left|\mathbf{d}(\mathbf{p}) \cdot \tilde{\mathbf{E}}_{\mathrm{X}}\left(\omega_{\mathbf{p}}\right)\right| \\
\varphi(\mathbf{p})= & \frac{1}{2} \mathbf{p}^{2}\left(t_{a}-t_{f}\right)+I_{p}\left(t_{a}-t_{0}\right)+ \\
& \phi_{d}(\mathbf{p})+\phi_{\mathrm{X}}\left(\omega_{\mathbf{p}}\right)+\frac{\pi}{2} .
\end{aligned}
$$

where $A(\mathbf{p})$ is the EWP amplitude, $\varphi(\mathbf{p})$ the full EWP phase, $\phi_{d}(\mathbf{p})=\arg [\mathbf{d}(\mathbf{p})]$ the dipole phase, $|\mathbf{d}(\mathbf{p})|$ the dipole amplitude and $\phi_{\mathrm{X}}\left(\omega_{\mathbf{p}}\right)$ the spectral phase of the XUV attosecond pulse which may contain, for example, an intrinsic chirp [44] besides of the XUV constant CEP itself. We define the frequency $\omega_{\mathbf{p}}$ axis as $\omega_{\mathbf{p}}=\frac{\mathbf{p}^{2}}{2}-\frac{\mathbf{p}_{0}^{2}}{2}$, with $\frac{p_{0}^{2}}{2}=$ $\omega_{\mathrm{X}}-I_{p}$. Here we will refer to this dipole phase $\phi_{d}(\mathbf{p})$ as residual EWP phase or for simplicity EWP phase. In addition, the term, $\tilde{\mathbf{E}}_{\mathrm{X}}\left(\omega_{\mathbf{p}}\right)=\mathbf{e}_{z} \int_{t_{0}}^{t_{f}} d t^{\prime} E_{0, \mathrm{X}}\left(t^{\prime}\right) e^{i \varphi_{\mathrm{X}}\left(t^{\prime}\right)} e^{i \omega_{\mathbf{p}}\left(t^{\prime}-t_{a}\right)}$, can easily be recognized as the FT of the XUV attosecond pulse envelope times its phase $e^{i \varphi_{\mathrm{X}}(t)}$ [139]. We note that Eq. (4.2) is derived by splitting the integral (4.1) into two parts with respect to the time $t_{a}$, which is the time at the maximum of the XUV attosecond envelope pulse. Generally, this does not mean the electron is ionized at time $t_{a}$.

From Eq. (4.2) we can easily identify that the emitted EWP carry information about the amplitude and phase of the XUV attosecond pulse. Also the structural dipole transition matrix element is coded into the EWP. Nevertheless, the attosecond and dipole phases are lost when the EWP momentum or energy distribution is measured in the lab.

Furthermore, it is important to note that the final continuum wavefunction $\psi_{\mathbf{p}}(\mathbf{r})=$ 
$\langle\mathbf{r} \mid \mathbf{p}\rangle=\frac{1}{\sqrt{2 \pi}} \exp (i \mathbf{p} \cdot \mathbf{r})$ is given by the $\mathrm{PW}$ of momentum $\mathbf{p}$. This often is a good approximation, i.e., when the photoelectron energy is high, however this is not generally the case. Then, we emphasize that the calculation of the complex dipole, $d_{z}(\mathbf{p})$, differs if PWs, $\psi_{\mathbf{p}}=\psi_{\mathbf{p}}^{(\mathrm{PW})}(\mathbf{r})$, or scattering waves, $\psi_{\mathbf{p}}=\psi_{\mathbf{p}}^{(\mathrm{SW})}(\mathbf{r})$, are used to compute it.
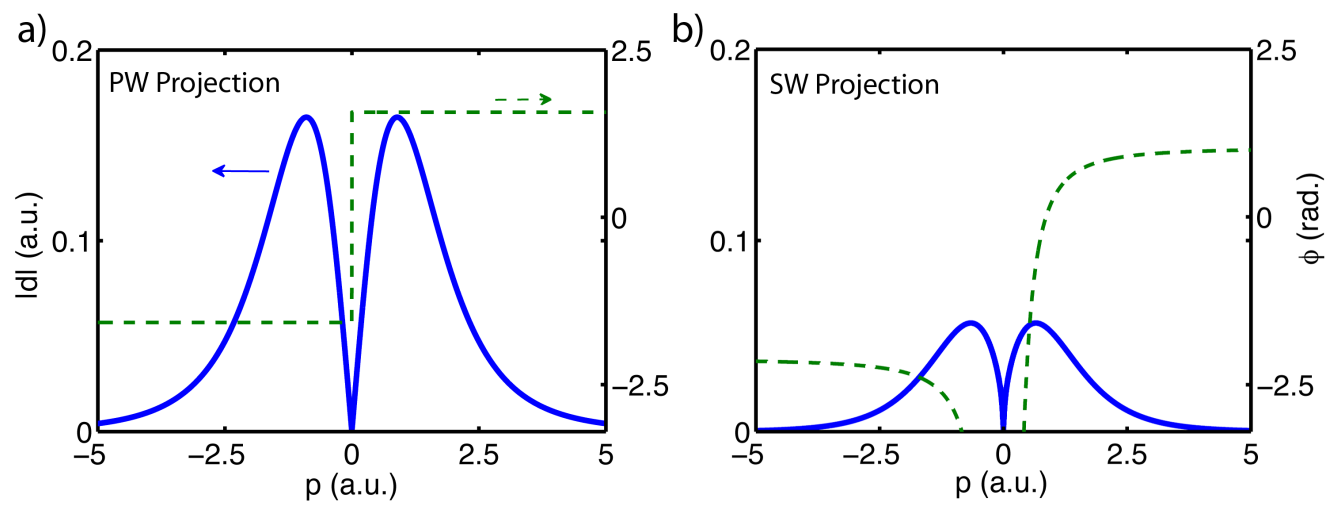

Figure 4.2: Structural dipole transition matrix element. In blue, the amplitude (left axis) and in green dashed the phase (right axis) of the bound-free dipole transition matrix element $d(p)$. Panel a) is calculated by projecting on the $\mathrm{PW}$ and panel b) on SW, respectively. In a) the complex dipole is computed for an hydrogen like atom to model the helium ion with ionization potential of $I_{p}=2.0$ a.u. The same in b) but for a $1 \mathrm{D}$ dimensional soft-core potential $V(z)$.

As an example, Fig. 4.2 shows the bound-free dipole transition matrix element computed via plane waves and scattering waves projections.

For the complex dipole depicted in a) we employ the analytical formula:

$$
d_{z}(\mathbf{p})=i\left(\frac{2^{7 / 2} \gamma^{5 / 2}}{\pi}\right) \frac{p_{z}}{\left(\gamma^{2}+\mathbf{p}^{2}\right)^{3}}
$$

with $\gamma^{2}=2 I_{p}[43,140]$, along $p_{z}$. For simplicity we will denote the momentum $p_{z}$ as $p$. We choose the ionization potential $I_{p}=2.0$ a.u., of the helium ion ground state which has a potential well $V_{0}(r)=-Z / r$ with $Z=2$. On the other hand Fig. $4.2 \mathrm{~b}$ ) depicts the same complex dipole but for a $1 \mathrm{D}$ soft-core Coulomb potential well $V_{0}(z)=-\frac{Z}{\sqrt{a+z^{2}}}$. We find that $Z=2$ and $a=0.5$ a.u. are the parameters which match the ionization potential of the $\mathrm{He}^{+}$.

In case of b), we numerically compute the ground state of the soft-core potential and then compute the complex dipole by projection on our 1D numerical SWs $\Psi_{p}(z)$ (for more detail about the SWs, see Section 2.2.1.4). The $z$-axis step is $\Delta z=0.01$ 
a.u. with a grid maximum $L_{z}=2400$ a.u. The momentum $p$-axis parameters are: $\Delta p=0.001$ a.u., and maximum $L_{p}=10$ a.u.

We find that while both methods yield a similar shape for the dipole amplitude (the only difference is a factor), the phases are completely different. This is so, because the scattering wave contains full information about the potential of the system.

We look now at the ionization by a SAP. By integrating the TDSE in 1D, we compute the full wavefunction $\Psi(z, t)$ and the EWP emitted, $a(p)=\Psi_{c}(p)$, to the continuum by the SAP. The Hamiltonian $H(t)$, which describes the electron interactions with the laser and the atom, in the velocity gauge, is defined by:

$$
H(t)=\frac{1}{2}\left[p_{z}+A_{\mathrm{X}}(t)\right]^{2}+V_{0}(z)
$$

where $A_{\mathrm{X}}(t)=-\int^{t} d t^{\prime} E_{\mathrm{X}}\left(t^{\prime}\right)$ is the vector potential of the XUV electric field $E_{\mathrm{X}}(t)$ and $p_{z}=-i \frac{\partial}{\partial z}$ denotes the canonical momentum operator. Then, to calculate the momentum distribution, $\mathrm{S}_{e}(p)=|a(p)|^{2}=\left|\Psi_{c}(p)\right|^{2}$, we project the total final wavefunction, $\Psi\left(z, t_{f}\right)$, onto a complete set of the scattering waves $\Psi_{p}(z)$ with asymptotic momentum $p$. This complex amplitude $a(p)$ reads:

$$
a(p)=\left\langle\Psi_{p} \mid \Psi\left(t_{f}\right)\right\rangle
$$

In order to compare the emitted EWP computed by both methods, we depict in Fig. 4.3 the amplitude and phase of the final momentum distributions. The ionization comes from the ground state of the $\mathrm{He}^{+}$, which has an ionization potential $I_{p}=2.0$ a.u.

The amplitude of the momentum distributions only differs by a factor. However, the EWP phases are completely different. In case of the TDSE model, the EWP phase is strongly dependent on the momentum while in the perturbative theory it is not.

Furthermore, we compare the dipole transition matrix element phase $\phi_{d}(p)$ to the EWP phase $\phi_{\mathrm{EWP}}(p)$, after removing the time dependent phase $\phi(p)=\frac{1}{2} p^{2}\left(t_{f}-t_{a}\right)+$ $I_{p}\left(t_{a}-t_{0}\right)+\phi_{0, X}$, with $\phi_{0, X}$, i.e., the XUV pulse carrier envelope phase. We note that the EWP phase is in very good agreement with the dipole phase.

Both methods demonstrate that the ionized EWP acquires the phase of the dipole transition matrix element in the photoemission process. This result is well known theoretically, however traditional observables in the lab do not have access to such information.

In this Section we have shown that an EWP carries out information about the 

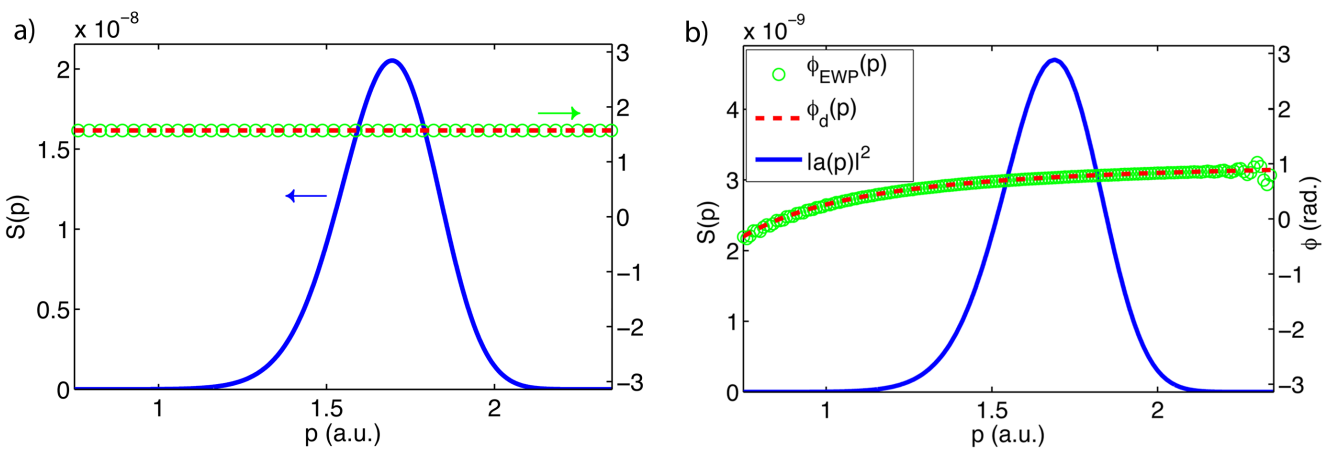

Figure 4.3: Attosecond electron wavepacket. The amplitude (blue line, left axis) and phase (green circles, right axis) of the final momentum distribution for the emitted EWP by a single XUV attosecond pulse starting from a helium ion ground state are depicted. Both methods, i.e., the first-order perturbative theory (a) and the exact numerical solution of the TDSE (b) are presented. For comparison, in the same plot, the dipole transition matrix element phase is shown in red dashed line. The XUV attosecond pulse parameters are: central frequency $\omega_{\mathrm{X}}=3.5$ a.u., and peak intensity $I_{\mathrm{X}}=10^{13} \mathrm{~W} / \mathrm{cm}^{2}$. The envelope is a gaussian with FWHM $=7.02$ a.u., $(170$ as $)$ and the CEP is $\frac{\pi}{2}$.

amplitude and phase of the dipole transition matrix element. In order to extract the dipole phase, we need a Quantum SPIDER. In the next Section we will show how two EWPs can easily be created by a train of two attosecond pulses. It will show that from the electron momentum interferogram fringes, the delay between the EWPs or the APT is extracted in analogy with the Optical SPIDER.

\subsubsection{Photoionization by two delayed attosecond pulses}

In the traditional optical SPIDER the information of the phase difference is directly encoded in the interferogram created by the two delayed copies of the laser pulse which one desires to characterize. Here, in analogy to the optical SPIDER, we describe how two delayed EWPs copies are constructed by the subsequent application of two XUV attosecond pulses.

Let us say that the electromagnetic fields of such two pulses are denoted by $\mathbf{E}_{\mathrm{X}}^{(1)}(t)$ and $\mathbf{E}_{\mathrm{X}}^{(2)}(t-\tau)$. The APT is then $\mathbf{E}_{\mathrm{X}}(t)=\mathbf{E}_{\mathrm{X}}^{(1)}(t)+\mathbf{E}_{\mathrm{X}}^{(2)}\left(t-\tau_{\mathrm{X}}\right)$. Here, $\tau_{\mathrm{X}}=t_{a}^{(2)}-t_{a}^{(1)}$ denotes the delay between the two XUV pulses with respect to the envelope maxima. According to the first-order perturbation theory Eq. (4.2), the complex amplitude transition $a(\mathbf{p})$ for the two coherent emitted EWPs reads: 


$$
a\left(\mathbf{p}, \tau_{\mathbf{x}}\right)=a_{1}(\mathbf{p})+a_{2}\left(\mathbf{p}, \tau_{\mathbf{x}}\right)
$$

with

$$
\begin{aligned}
a_{1}(\mathbf{p}) & =A_{1}(\mathbf{p}) e^{i \varphi_{1}(\mathbf{p})}, \\
a_{2}\left(\mathbf{p}, \tau_{\mathbf{x}}\right) & =A_{2}(\mathbf{p}) e^{i \varphi_{2}\left(\mathbf{p}, \tau_{\mathbf{x}}\right)},
\end{aligned}
$$

where $A_{j}(\mathbf{p})$ and $\varphi_{j}(\mathbf{p})$ are the EWP amplitudes and phases which are defined according to the formulae given in Eq. (4.2). Here $j=1,2$ counts the two EWPs. The two delayed copies will produce an interferogram pattern if the central frequencies $\omega_{(j), \mathrm{X}}$ of the XUV pulses are the same or with similar values between them. This interferogram $\mathrm{S}_{e}\left(\mathbf{p}, \tau_{\mathrm{x}}\right)=\left|a\left(\mathbf{p}, \tau_{\mathbf{x}}\right)\right|^{2}$ is defined as the square amplitude in Eq. (4.6):

$$
\begin{aligned}
\mathrm{S}_{e}\left(\mathbf{p}, \tau_{\mathrm{x}}\right) & =\left|a_{1}(\mathbf{p})+a_{2}\left(\mathbf{p}, \tau_{\mathrm{x}}\right)\right|^{2} \\
& =\left|A_{1}(\mathbf{p})\right|^{2}+\left|A_{2}(\mathbf{p})\right|^{2}+2 A_{1}(\mathbf{p}) A_{2}(\mathbf{p}) \cos \left[\Delta \varphi\left(\mathbf{p}, \tau_{\mathrm{x}}\right)\right] .
\end{aligned}
$$

In our notation, $\Delta \varphi\left(\mathbf{p}, \tau_{\mathbf{x}}\right)=\left(\frac{1}{2} \mathbf{p}^{2}+I_{p}\right) \tau_{\mathbf{x}}+\pi$, is the phase difference between both delayed emitted EWPs. Note that the $\pi$ phase difference comes from the fact that the $\mathrm{XUV}$ attosecond pulse is in counter-phase.

Note that this simple Eq. (4.9) shows us that the dipole phase difference, $\Delta \phi_{d}(\mathbf{p})$, as well as the XUV attosecond pulse phase difference, $\Delta \phi_{\mathrm{X}}\left(\omega_{\mathbf{p}}\right)$, are missed in the measurement process of the two EWPs.

As an example, we calculate the two delayed launched EWPs from the $\mathrm{He}^{+}$by an APT of two pulses. To show how the interference pattern changes as a function of the delay $\tau_{\mathrm{x}}$, two different delays $\left(\tau_{\mathrm{x}}=T_{0} / 2\right.$ and $\left.\tau_{\mathrm{x}}=T_{0}\right)$ have been employed. These calculations are done by the numerical integration of Eq. (4.6) for the same system described in the last Section. The results are depicted in Fig. 4.4.

The panels a-b) show the time evolution of the momentum distribution when the attosecond pulses interact with the system. It is clear that the first EWP is emitted about the middle of $E_{\mathrm{X}}^{(1)}(t)$ and immediately after, the second EWP is launched to the continuum by the second attosecond pulse $E_{\mathrm{X}}^{(2)}\left(t-\tau_{\mathrm{X}}\right)$ to the same final momenta. While the delay $\tau_{\mathrm{x}}$ increases, the distance between two consecutive minima (or maxima) decreases. From Eq. (4.9) the interferogram minima (or zeros) occur when the phase difference is $\Delta \varphi\left(p_{n}, \tau_{\mathrm{x}}\right)=\pi(2 n+1)$. Here $n=0,1, \ldots$ denotes the nodes or zeros in the 

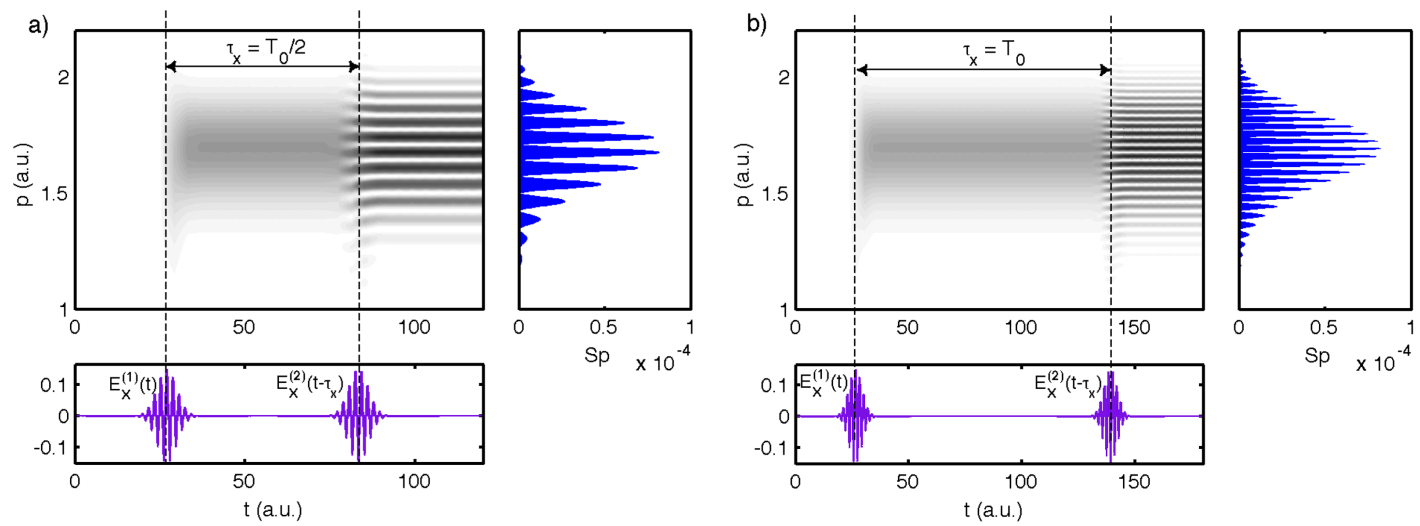

Figure 4.4: Attosecond EWPs interferences. Panels a) and b) show the two delayed EWPs momentum distributions, $\mathrm{S}_{e}(p, t)$, (gray color scale, white the lowest and black the largest ones) as a function of time, the final momentum distribution depicted in blue area and the APT in violet area for two different time delays, $\left(\tau_{\mathrm{x}}=T_{0} / 2\right.$ and $\left.\tau_{\mathrm{x}}=T_{0}\right)$, between each attosecond pulse. In our notation, $T_{0}=2.6 \mathrm{fs}$, is the period cycle of an IR $(800 \mathrm{~nm})$ laser field. We have performed these calculations by integrating the derived Eq. (4.6). The attosecond pulse parameters are the same as those used in Fig. 4.3, but here the CEP difference is $\pi$. The momentum grid parameters are $\Delta p=0.001$ a.u., with a maximum grid size $L_{p}=12.5$ a.u.

momentum distribution. We can easily verify that for two consecutive momenta, $p_{n}$ and $p_{n+1}$, corresponding to the zeros of the final momentum interferogram the phase difference is:

$$
\Delta E \tau_{\mathrm{x}}=2 \pi
$$

with $\Delta E=\frac{1}{2} p_{n+1}^{2}-\frac{1}{2} p_{n}^{2}$. The numerical results perfectly match the relation written in Eq. (4.10) for the two different delays illustrated in a) and b).

It is important to point out that this method is suitable for measuring the time delay $\tau_{\mathrm{x}}$ between two EWPs or attosecond pulses. However, the dipole phase is missed and an extra analysis has to be employed in order to retrieve it.

The attosecond streak camera has demonstrated that an EWP can be shifted in momentum or energy distribution. In the next Section, we will describe how a weak and ultrashort IR laser pulse induces a momentum shift over the whole emitted EWP by the XUV radiation. Then, we can apply it as a process to induce a spectral or momentum shearing like the one used in the optical SPIDER technique. The nonlinear interaction frequency sum introduces a spectral shearing into the frequencies of the 
pulses to be characterized.

\subsubsection{Photoionization in presence of an infrared laser}

The ionization by an SAP, in the presence of a moderate IR laser pulse, can be described by considering the bound-free transition amplitude $a(\mathbf{p}, \tau)=\left\langle\mathbf{p}\left|U\left(t_{f}, t_{0}\right)\right| \Psi_{0}\right\rangle$ that describes the transition from the initial state $\left|\Psi_{0}\right\rangle$ at time $t_{0}$, before the pulses arrives, to a final continuum state $|\mathbf{p}\rangle$ at the detection time $t_{f}$. The system evolves according to the operator $U\left(t_{f}, t_{0}\right)$. In our notation $\tau$ is a parameter that defines the time delay between the XUV and the IR laser pulses. An approximated treatment of this transition is obtained by using perturbation theory for the XUV-induced ionization and the SFA for the coupling to the IR laser field as it was derived in Section 2.1.6.2:

$$
a(\mathbf{p}, \tau)=i \int_{t_{0}}^{t_{f}} d t^{\prime} e^{i\left[I_{p}\left(t^{\prime}-t_{0}\right)-\frac{1}{2} \int_{t^{\prime}}^{t_{f}}\left(\mathbf{p}+\mathbf{A}_{\mathbf{L}}\left(t^{\prime \prime}\right)\right)^{2} d t^{\prime \prime}\right]} \mathbf{E}_{\mathbf{X}}\left(t^{\prime}-\tau\right) \cdot \mathbf{d}\left(\mathbf{p}+\mathbf{A}_{\mathrm{L}}\left(t^{\prime}\right)\right) .
$$

Here $\mathbf{A}_{\mathrm{L}}(t)=-\int{ }^{t} \mathbf{E}_{\mathrm{L}}\left(t^{\prime}\right) d t^{\prime}$ is the vector potential of the IR field $\mathbf{E}_{\mathrm{L}}(t)$. We consider a linearly polarized IR laser pulse and pulses linearly XUV polarized along the same $z$-axis. The time delay $\tau$ in the Eq. (4.11), between the XUV and IR pulses, is defined with respect to the maximum of the pulse envelopes. Positive (negative) time delay means that the IR arrives before (after) that the XUV attosecond pulse. Fig. 4.5) shows an example where we observe the time delay line between the IR and XUV pulses.

Within the SFA approach, the final momentum $|\mathbf{p}\rangle$ is approximated by a PW and the dipole transition matrix element is defined as $\mathbf{d}\left(\mathbf{p}+\mathbf{A}_{\mathrm{L}}(t)\right)=-\mathbf{e}_{z}\left\langle\mathbf{p}+\mathbf{A}_{\mathrm{L}}(t)|z| \Psi_{0}\right\rangle$. According to Eq. (4.11), the ionization is driven by the XUV pulse only, while the IR laser pulse contributes to the phase of the EWP and changes its momentum from $\mathbf{p}+\mathbf{A}_{\mathrm{L}}(t)$ at the time of ionization to $\mathbf{p}_{f}$ at the final time.

An alternative way to compute the integral of Eq. (4.11) is its differential formulation [116] described in Eq. (2.95) of Section 2.2.3. This expression appears to be very advantageous because it can be integrated by using any algorithm for differential equations, i.e., fourth-order Runge-Kutta, and does not require to deal with the saddle point approximation. In addition, the calculation can be parallelized easily using the MPI [141] paradigm or multiple threads using Graphics Processing Units (GPUs) under CUDA [142].

A convenient approximation can be used when the SAP is much shorter than the period $T_{0}$ of the IR pulse. The final momentum-space wave function is a product of an 
amplitude and a phase factor, similar to the derivation presented in Section 4.2.1. For an SAP centered at $\tau$ with respect to the maximum of the IR envelope, we write:

$$
a(\mathbf{p}, \tau)=A(\mathbf{p}, \tau) e^{i \phi\left(\mathbf{p}, \tau, t_{f}\right)}
$$

with

$$
\begin{aligned}
A(\mathbf{p}, \tau)= & \frac{1}{2}\left|\mathbf{d}\left(\mathbf{p}+\mathbf{A}_{\mathrm{L}}(\tau)\right) \cdot \mathbf{E}_{0, \mathrm{X}}\left(\omega_{\mathbf{p}}\right)\right| \\
\phi\left(\mathbf{p}, \tau, t_{f}\right)= & \phi_{\mathbf{d}\left(\mathbf{p}+\mathbf{A}_{\mathrm{L}}(\tau)\right)+I_{p}\left(\tau-t_{0}\right)} \\
& -\frac{1}{2} \int_{\tau}^{t_{f}}\left[\mathbf{p}+\mathbf{A}_{\mathrm{L}}\left(t^{\prime \prime}\right)\right]^{2} d t^{\prime \prime} \\
& +\phi_{\mathrm{X}}\left(\omega_{\mathbf{p}}\right)+\phi_{\mathrm{LIC}}(\mathbf{p}, \tau)+\frac{\pi}{2} .
\end{aligned}
$$

Here $\mathbf{d}\left(\mathbf{p}+\mathbf{A}_{\mathrm{L}}(\tau)\right)=\mathbf{e}_{z} d_{z}\left(\mathbf{p}+\mathbf{A}_{\mathrm{L}}(\tau)\right)$ is the component of the dipole along the polarization $z$-axis. The expression $E_{0, \mathrm{X}}\left(\omega_{\mathbf{p}}\right)=\left|E_{0, \mathrm{X}}\left(\omega_{\mathbf{p}}\right)\right| e^{i \phi_{\mathrm{X}}\left(\omega_{\mathbf{p}}\right)}$ is the FT of $\tilde{E}_{0, \mathrm{X}}(t) e^{i \varphi_{\mathrm{X}}(t)}$ as a function of $\omega_{\mathbf{p}}$, which is defined by:

$$
\omega_{\mathbf{p}}=\frac{1}{2}\left[\mathbf{p}+\mathbf{A}_{\mathrm{L}}(\tau)\right]^{2}-\frac{p_{0}^{2}}{2}
$$

where $\frac{p_{0}^{2}}{2}=\omega_{\mathrm{X}}-I_{p}$. In addition, we note that the central energy $E_{c}=\frac{1}{2} p_{\mathrm{c}}^{2}$ at the maximum of any final momentum distribution (as result of the solution of Eq. (4.11)), may be easily computed when the argument of the distribution is zero ${ }^{1} \omega_{\mathbf{p}_{\mathbf{c}}}=0$. This means the electron momentum distribution of the final resulting EWP will be centered at the momentum $p_{\mathrm{c}}=p_{0}-A_{\mathrm{L}}(\tau)$, which is the same result commonly obtained via the streaking effect, i.e., $[58,59]$. It is important to point out that this momentum shift, $p_{\mathrm{c}}-p_{0}=-A_{\mathrm{L}}(\tau)$, provides a way to introduce the spectral shearing between two delayed EWPs. As we will see, this is one of the most important parts in the QSPIDER implementation.

To obtain Eq. (4.13), we have expanded the phase integral in the exponent of Eq. (4.11) to first order in $t^{\prime}$ around $\tau$, with $\mathbf{A}_{\mathrm{L}}\left(t^{\prime}\right) \approx \mathbf{A}_{\mathrm{L}}(\tau)-\mathbf{E}_{\mathrm{L}}(\tau)\left(t^{\prime}-\tau\right)$. This is well justified because, according to the stationary phase condition, the main contribution in Eq. (4.11) will occur around the maximum of the XUV attosecond pulse. In addition,

\footnotetext{
${ }^{1}$ For instance, a gaussian amplitude $f\left(\omega_{\mathbf{p}}\right)=f_{0} e^{-\omega_{\mathbf{p}}^{2} / \sigma^{2}}$, then the gaussian is centered at $\omega_{\mathbf{p}}=$ $\frac{1}{2}\left[\mathbf{p}+\mathbf{A}_{\mathrm{L}}(\tau)\right]^{2}-\frac{p_{0}^{2}}{2}=0$.
} 
we assume that the IR intensity is weak enough and then the IR does not ionize the system. We further neglect the changes in the dipole due to the expansion and approximate $\mathbf{d}\left(\mathbf{p}+\mathbf{A}_{\mathrm{L}}\left(t^{\prime}\right)\right) \approx \mathbf{d}\left(\mathbf{p}+\mathbf{A}_{\mathrm{L}}(\tau)\right)$.

In the Eq. (4.14) $\phi_{\mathrm{X}}\left(\omega_{\mathbf{p}}\right)$ is the spectral phase of the attosecond pulse as it is mentioned in Section 4.2.1. The phase $\phi_{\mathrm{LIC}}(\mathbf{p}, \tau)$ describes the Laser Induced Chirp of the EWP caused by the variations of the IR field around time $\tau$, that locates the maximum of the XUV with respect to the IR. Assuming a Gaussian pulse shape $\tilde{E}_{0, X}(t) \sim e^{-\alpha t^{2}}$, this phase is given by:

$$
\begin{aligned}
\phi_{\mathrm{LIC}}(\mathbf{p}, \tau)= & -\frac{1}{2} \arctan \left[\frac{\Gamma(\mathbf{p}, \tau)}{2 \alpha}\right]+ \\
& \frac{2 \Gamma(\mathbf{p}, \tau)}{(4 \alpha)^{2}+[2 \Gamma(\mathbf{p}, \tau)]^{2}}\left[\frac{1}{2}\left(\mathbf{p}+\mathbf{A}_{\mathrm{L}}(\tau)\right)^{2}-\frac{p_{0}^{2}}{2}\right]^{2},
\end{aligned}
$$

with $\Gamma(\mathbf{p})=\left[\mathbf{p}+\mathbf{A}_{\mathrm{L}}(\tau)\right] \cdot \mathbf{E}_{\mathrm{L}}(\tau)$. The LIC phase is responsible for the different widths of the EWP in the position space and this is a measure of the attosecond pulse duration with respect to the variations of the vector potential. For short SAPs $(<200$ as $)$ and modest intensity $\left(I_{\mathrm{X}}<10^{13} \mathrm{~W} / \mathrm{cm}^{2}\right)$ the effects of the LIC phase are negligible. This phase, Eq. (4.16), depends on the value of the electric field at ionization time $\tau$ and it is zero if $\mathbf{E}_{\mathrm{L}}(\tau)=\mathbf{0}$. A similar phase exists in the case of the optical SPIDER when an insufficiently chirped ancilla is used [143]. This phase will become relevant for streaking and interferometric measurements because it can be larger than the dipole phase.

We compute the ionization probability as a function of the delay $\tau$, by numerical integration of the Eq. (4.11) in order to illustrate the streaking effect. We choose the same ground state of the $\mathrm{He}^{+}$system utilized in the Section 4.2.1. The results are depicted in Fig. 4.5. We compare the emitted EWP by the XUV source in presence of the moderated IR field for different time delays (see Fig. 4.5b-d) to a single EWP in the case that only the XUV (see Fig. 4.5a) is acting on the system. We note that the final EWP momentum distribution is streaked by the IR laser field and this streak effects follow exactly the negative values of the IR vector potential $\Delta p=-A_{\mathrm{L}}(\tau)$ at the time delay $\tau$. This so-called streaking effect will occur under two conditions: First, the XUV attosecond pulse has to be much shorter than one IR laser cycle and second, the IR intensity $\left(\sim 10^{10}-10^{12} \mathrm{~W} / \mathrm{cm}^{2}\right)$ requires to be weak enough to not further ionize the atom.

The streaking trace is the energy or momentum distribution, $\mathrm{S}(p, \tau)$, for the electrons ionized by a SAP in presence of a weak IR for all the delays, $\tau$, between the two pulses. 

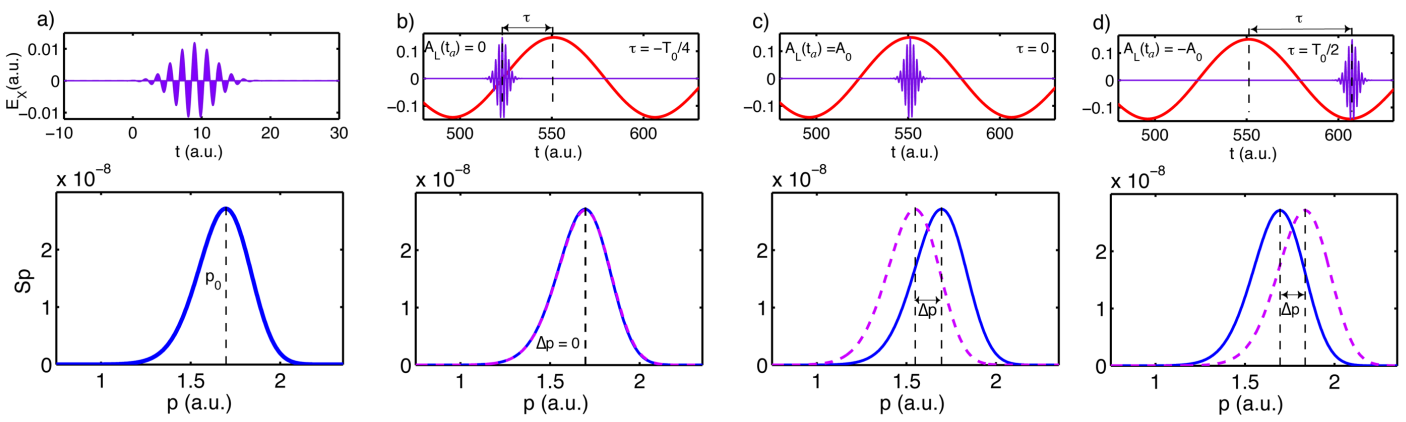

Figure 4.5: Photoionization in presence of an IR laser. In the panel a) we plot the XUV attosecond pulse (violet area) and the final momentum distribution (blue line) of the emitted EWP by the XUV radiation. Black vertical dashed line indicates the momentum $p_{0}$ at the maximum of the distribution. In the upper plots of the next panels b-d), the two colors pulses, the XUV and the vector potential of the IR (red line) are shown for three different time delays $\tau=-T_{0} / 4, \tau=0$ and $\tau=T_{0} / 2$. The lower plots depict the final photoelectron momentum distributions, $\mathrm{S}_{e}(p)=|a(p, \tau)|^{2}$, (magenta dashed line) for the ionization driven by the XUV pulse in presence of the weak IR laser field compared to the case where only the XUV ionizes the system. Black vertical dashed lines show the momentum shift, $\Delta p=p_{\mathrm{c}}-p_{0}=$ $-A_{\mathrm{L}}(\tau)=-A_{\mathrm{L}}\left(t_{a}\right)$, induced by the laser IR field. Here, $A_{0}$, denotes the maxima of the IR vector potential $A_{\mathrm{L}}(t)$. These calculations were done by integrating numerically Eq. (4.11). The XUV attosecond pulse parameters are the same than those utilized in Fig. 4.3. The IR laser electromagnetic field frequency is $\omega_{0}=0.057$ a.u. ( $800 \mathrm{~nm}$ of wavelength), its peak intensity $I_{0}=2.5 \times 10^{12} \mathrm{~W} / \mathrm{cm}^{2}$, its envelope pulse is a gaussian with $\mathrm{FWHM} \sim 8.0 \mathrm{fs}$ and its $\mathrm{CEP}=0$. 
This is the basic mechanism that explains the streak camera which has been successfully employed in severals attosecond experiments [3, 6, 59].

In conclusion, according to these analytical and numerical results for the streak effect conditions, we have demonstrated that the photoelectron spectrum generated by an XUV attosecond pulse in presence of an IR laser, is momentum shifted.

The next Section will be devoted to study how this momentum or spectral shearing is induced when two EWP copies are emitted to the continuum by the subsequent application of two delayed XUV attosecond pulses in presence of an weak IR laser field.

\subsubsection{Attosecond electron wave packet interferometry}

In Section 4.2.2, we described how two delayed copies of the EWP can be launched to the continuum by two SAPs. However, this method missed the dipole phase difference between the two emitted EWPs. In this Section, we will show how the induced momentum shift by the IR laser pulse can introduce the desirable spectral shearing on the final momentum interferogram.

In order to implement the QSPIDER technique, two delayed copies of the EWP with a relative shear or momentum shift between them are needed to construct an interferogram, which will contain the dipole phase difference. To this end, we use an APT with two pulses centered at $\tau_{1}$ and $\tau_{2}$, respectively in the presence of a weak IR laser pulse. The two pulses generate two EWPs which are relatively delayed to each other by the separation of the pulses in the train $[62,144]$.

The weak IR laser pulse streaks each of the EWPs by the value of the vector potential at the ionization time [58] to produce a relative streaking $\Delta \mathbf{A}_{\mathrm{L}}=\mathbf{A}_{\mathrm{L}}\left(\tau_{2}\right)-\mathbf{A}_{\mathrm{L}}\left(\tau_{1}\right)$ between the two copies. The streaked and delayed copies produce an interferogram in the final momentum distribution which is conceptually equivalent to the interferogram of the optical SPIDER technique. According to the SFA, the interferogram is:

$$
\begin{aligned}
\left|a\left(\mathbf{p}, \tau_{1}, \tau_{2}\right)\right|^{2}= & \left|A_{1}\left(\mathbf{p}, \tau_{1}\right) e^{-i \phi\left(\mathbf{p}, \tau_{1}, t_{f}\right)}+A_{2}\left(\mathbf{p}, \tau_{2}\right) e^{-i \phi\left(\mathbf{p}, \tau_{2}, t_{f}\right)}\right|^{2} \\
= & A_{1}\left(\mathbf{p}, \tau_{1}\right)^{2}+A_{2}\left(\mathbf{p}, \tau_{2}\right)^{2} \\
& +2 A_{1}\left(\mathbf{p}, \tau_{1}\right) A_{2}\left(\mathbf{p}, \tau_{2}\right) \cos \left(\Delta \phi_{21}\right)
\end{aligned}
$$

Here, $A_{1}\left(\mathbf{p}, \tau_{1}\right)$ and $A_{2}\left(\mathbf{p}, \tau_{2}\right)$, are the (real) amplitudes for each of the EWPs. The interference term in Eq. (4.17) is governed by the relative phase 


$$
\begin{aligned}
\Delta \phi_{21}= & \Delta \phi_{d}\left(\mathbf{p}, \tau_{1}, \tau_{2}\right)+\frac{1}{2} \int_{\tau_{1}}^{\tau_{2}} d t^{\prime \prime}\left(\mathbf{p}+\mathbf{A}_{\mathrm{L}}\left(t^{\prime \prime}\right)\right)^{2} \\
& +I_{p} \Delta \tau+\Delta \phi_{\mathrm{X}}\left(\mathbf{p}, \tau_{1}, \tau_{2}\right)+\Delta \phi_{\mathrm{LIC}}\left(\mathbf{p}, \tau_{1}, \tau_{2}\right) .
\end{aligned}
$$

Where, $\Delta \tau=\tau_{2}-\tau_{1}$, is the delay between the two attosecond pulses and $\Delta \phi_{d}\left(\mathbf{p}, \tau_{1}, \tau_{2}\right)=$ $\phi_{d\left(\mathbf{p}+\mathbf{A}_{\mathrm{L}}\left(\tau_{2}\right)\right)}-\phi_{d\left(\mathbf{p}+\mathbf{A}_{\mathrm{L}}\left(\tau_{1}\right)\right)}$ is the phase difference of the dipole transition matrix element.

In this approximation the interference depends on five terms. The first one, $\Delta \phi_{d}\left(\mathbf{p}, \tau_{1}, \tau_{2}\right)$, is related to the phase of the dipole transition matrix element. The second term is the Volkov phase related to the different time spent in the continuum by the two EWPs. The third term is the accumulated phase difference in the initial state before ionization, which we call atomic phase. The two last terms are related to the spectral phases of the attosecond pulses, which also contain the carrier envelope phase difference and the laser-induced chirp, respectively.

The interference between the two streaked EWPs depends on the emission direction. Assuming a small collection angle around the polarization direction, we can restrict ourselves to 1D modelling, i.e., we focus on electrons emitted along the polarization axis and in a defined left and right direction.

As an example, Fig. 4.6 shows a comparison between the time evolution of the momentum distributions when the two delayed XUV attosecond pulses create two delayed EWP copies (a), and when the two XUV pulses in presence of a weak IR laser field launch the two EWP copies to the continuum (b).

We note that the time evolution of the momentum distributions in a) differs from b). In particular, panel b) shows that the first ionization event by the XUV pulse $E_{\mathrm{X}}^{(1)}$, is strongly upper shifted, to higher momentum values, and the second one by the $E_{\mathrm{X}}^{(2)}$, is down shifted, to smaller momentum values, with respect to the case where there is no IR (see Fig. 4.6a)). These shifted EWPs lead to a final momentum interferogram which differs from the case when there is no IR field, as it is expected. According to Eq. (4.18) this disagreement is mainly caused by the relative phase difference induced by the vector potential of the IR laser field.

We point out that under the SFA model, this final interferogram contains full information about the dipole phase difference, LIC phase difference and the attosecond phase difference if the attosecond pulses are chirped.

We have completed all the required elements for the QSPIDER implementation, namely: two delayed EWP copies, a relative spectral shearing and a final interferogram. 

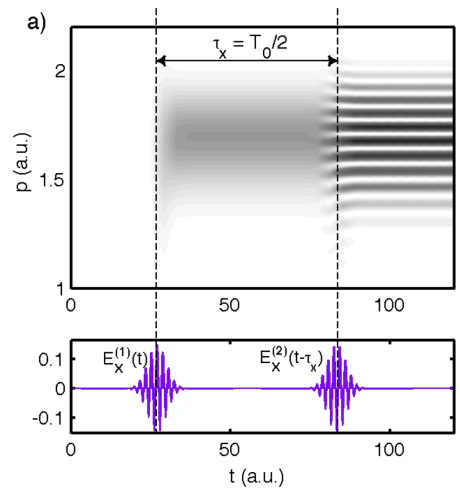
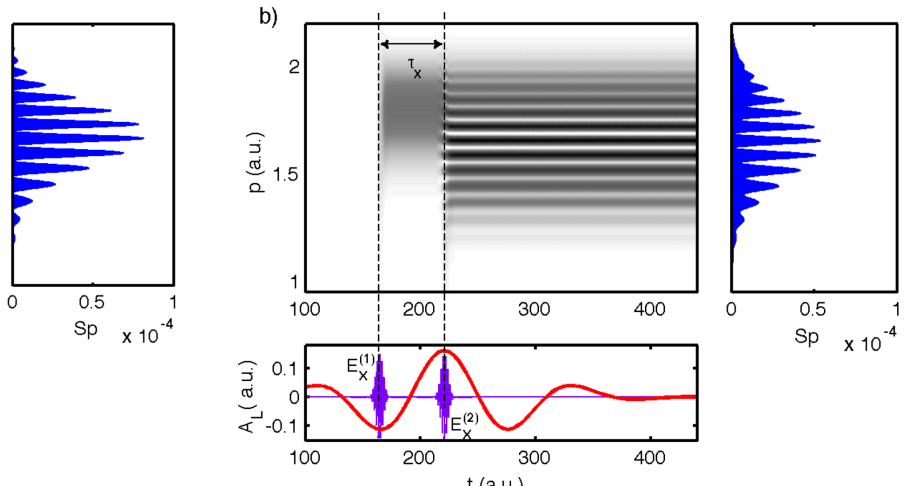

Figure 4.6: Attosecond EWP interferometry in presence of an IR laser. In panel a) we plot the time evolution of the electron momentum distribution (gray scale color) when two delayed $\left(\tau_{\mathrm{x}}=T_{0} / 2\right) \mathrm{XUV}$ attosecond pulses, $E_{\mathrm{X}}^{(1)}$ and $E_{\mathrm{X}}^{(2)}$ (see violet area, in the lower graphs), ionize the helium ion atom according to the perturbative theory. The final momentum interferogram is also depicted in the blue area. Panel b) shows the same, but here the ionization is performed in presence of a weak IR laser field. The XUV attosecond pulses are centered at, $\tau_{1}=-T_{0} / 2$ and $\tau_{2}=0$, with respect to the IR envelope max. The relative delay $\tau_{\mathrm{x}}=\tau_{2}-\tau_{1}$, between the XUV pulses is $\tau_{\mathrm{x}}=T_{0} / 2$. The maxima of the XUV envelopes are located at a negative and positive maxima of the IR vector potential $A_{\mathrm{L}}(t)$, as it is indicated by the black dashed vertical lines in b). In the lower plot, the red line depicts the IR vector potential $A_{\mathrm{L}}(t)$, and the APT, too. The attosecond pulse parameters are those used in Fig. 4.4. We emphasize that APT is Fourier limit, i.e., the attosecond chirp is zero. The IR central frequency is $\omega_{0}=0.057$ a.u., its peak intensity $I_{0}=2.5 \times 10^{12} \mathrm{~W} / \mathrm{cm}^{2}$, its envelope is a guassian function with $\mathrm{FWHM} \sim 4.0 \mathrm{fs}$, and its $\mathrm{CEP}=0 \mathrm{rad}$. The momentum grid parameters are $\Delta p=0.001$ a.u., and the momentum length $L_{p}=12.5$ a.u. 


\section{ELECTRON WAVE PACKET INTERFEROMETRY}

We emphasize that, so far, our derivation is based on the SFA model. This theoretical approach does not take into account the possible phase shift of the continuum scattering states and the possible IR laser-Coulomb coupling in case of Coulombic potentials. As it was stated before, we will employ the numerical integration of the TDSE model as well, for our QSPIDER implementation, considering that it is important to model the phase difference introduced by the Coulomb laser coupling. To this end, the next Section will be devoted to describe the coupling between the IR laser and the Coulomb potential by employing the eikonal approach.

\subsubsection{Coulomb laser coupling phase}

The theory outlined above is suitable only for systems that are well described by the SFA, i.e., potentials without a Coulomb tail or short-range ones. For Coulombic potentials, the separation of the electron dynamics into the two steps, ionization and interaction with the laser field, is problematic because the electron interacts with the laser field and the long-range potential simultaneously. The long-range character is also reflected in the field-free continuum states that are needed in the dipole transition matrix elements. For a potential $V(z)$ with asymptotic behavior $-Z /|z|$, the positivemomentum component of a continuum state $\Psi_{p}(z)$ for the asymptotic momentum $p>0$ behaves as:

$$
\Psi_{p}(z) \approx \exp [i p z+i Z \ln (2 p z) / p] \quad \text { for } z \rightarrow \infty .
$$

We will follow the eikonal approach of Ivanov and coworkers [145] in order to treat the Coulomb-laser coupled dynamics. For the purpose of calculating the time-evolution of the eikonal state, we assume an electron trajectory starting at a distance $z_{0}$ and moving outwards [146]. One finds that the position representation of the continuum state after evolving from the time of ionization $\tau$ to the final time $t_{f}$ is:

$$
\begin{aligned}
\left\langle z\left|U\left(t_{f}, \tau\right)\right| p+A_{\mathrm{L}}(\tau)\right\rangle= & e^{i p z} e^{-i \int_{\tau}^{t_{f}} d t\left(p+A_{\mathrm{L}}(t)\right)^{2} / 2} \\
& \times e^{-i \int_{\tau}^{t_{f}} d t V\left(z_{0}+p(t-\tau)+\int_{\tau}^{t} d t^{\prime} A_{\mathrm{L}}\left(t^{\prime}\right)\right)} \\
& \times e^{-\frac{i}{p+A_{\mathrm{L}}(\tau)} \int_{\mathrm{ref}}^{z_{0}} d l V(l)}
\end{aligned}
$$

where $|p\rangle$ denotes the eikonal continuum state with asymptotic momentum $p$ and 
$U\left(t_{f}, \tau\right)$ is the approximated time evolution operator. Since we are primarily interested in the phase correction due to the laser-Coulomb coupling, we have omitted the amplitude correction of the wave function in Eq. (4.20). In the following, we assume that the initial position is $z_{0}=1 /\left[2\left(p+A_{\mathrm{L}}(\tau)\right][146]\right.$ and furthermore we set $z_{0}=z_{\text {ref }}$ in accordance with the boundary condition of Eq. (4.19). Thus, the last phase factor in Eq. (4.20) is equal to 1. After a substitution $s=z_{0}+p(t-\tau)$ and inserting a Coulomb potential $V(z)=-Z /|z|$ we obtain, for $z>0$, that:

$$
\begin{aligned}
\left\langle z\left|U\left(t_{f}, \tau\right)\right| p+A_{\mathrm{L}}(\tau)\right\rangle= & e^{i p z} e^{-i \int_{\tau}^{t_{f}} d t\left(p+A_{\mathrm{L}}(t)\right)^{2} / 2} \\
& \times e^{\frac{i Z}{p} \int_{z_{0}}^{z_{f}} d s\left(s+\int_{\tau}^{\tau+\left(s-z_{0}\right) / p} d t^{\prime} A_{\mathrm{L}}\left(t^{\prime}\right)\right)^{-1}}
\end{aligned}
$$

where $z_{f}=z_{0}+p\left(t_{f}-\tau\right)$.

We consider two EWPs with the same asymptotic momentum $p$, but launched at two different times $\tau_{1}, \tau_{2}$. In the limit $t_{f} \rightarrow \infty$, we find that the two wave functions differ by a relative phase given by

$$
\begin{aligned}
\Delta \phi_{\mathrm{VC}}\left(p, \tau_{1}, \tau_{2}\right)= & \frac{1}{2} \int_{\tau_{1}}^{\tau_{2}} d t\left[p+A_{\mathrm{L}}(t)\right]^{2} \\
& +\lim _{z_{f} \rightarrow \infty}\left[\phi_{\mathrm{C}}\left(p, \tau_{2}\right)-\phi_{\mathrm{C}}\left(p, \tau_{1}\right)\right], \\
\phi_{\mathrm{C}}\left(p, \tau_{j}\right)= & \frac{Z}{p} \int_{z_{0, j}}^{z_{f}} \frac{d s}{s+\int_{\tau_{j}}^{\tau_{j}+\left(s-z_{0, j}\right) / p} d t^{\prime} A_{\mathrm{L}}\left(t^{\prime}\right)}
\end{aligned}
$$

where $z_{0, j}=1 / 2\left[p+A_{\mathrm{L}}\left(\tau_{j}\right)\right]$ for $j=1,2$. We conclude that in the case of Coulombic potentials, Eq. (4.18) should be replaced by the Coulomb-corrected phase difference

$$
\Delta \phi_{21}=\Delta \phi_{d}+\Delta \phi_{\mathrm{VC}}+I_{p} \Delta \tau+\Delta \phi_{\mathrm{X}}+\Delta \phi_{\mathrm{LIC}}
$$

where we have omitted the arguments for simplicity in the notation.

Finally, the next Section will be focused on describing how the QSPIDER technique is implemented with all the tools explained above. 


\subsubsection{QSPIDER implementation}

As previously mentioned, QSPIDER is a quantum implementation of the SPIDER technique. As it was clearly demonstrated in the last Section 4.2.4, the interference of two delayed EWPs led to an interferogram pattern in the electron momentum distribution, where the relevant phase difference $\Delta \phi_{21}$ is given by Eq. (4.18) or Eq. (4.23).

The technique first measures the phase difference $\Delta \phi_{21}$. For metrology applications, as suggested by Quere et al. [62], the spectral phase of the XUV pulse can be recovered by reconstructing the temporal shape of the XUV pulse. This is possible if $\Delta \phi_{d}$ and the Coulomb corrections are known. In [62] it was assumed that these two phases vanish, which is not the case for a real atom. In contrast, our aim is to retrieve both the amplitude and phase of the dipole transition matrix element, assuming that the XUV field is well known. This also requires the knowledge of quantities such as the relative shear and the delay between the pulses. All of these quantities can be measured and used as input information for our QSPIDER algorithm.

The scheme for measuring $\Delta \phi_{21}$ is depicted in Fig. 4.7. The panel c) shows a typical momentum spectrum as produced by the applied fields shown in panel a). This interferogram carries out information on the amplitude and phase of the EWPs.

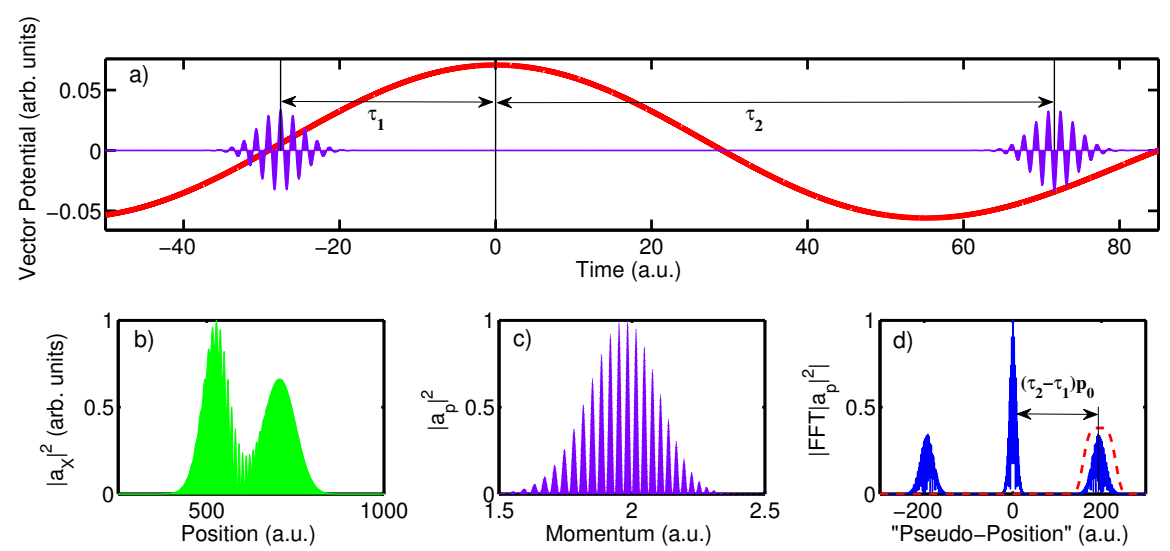

Figure 4.7: General idea of the QSPIDER technique. a) Two attosecond pulses (violet area) in the presence of a weak vector potential of an IR laser (red solid line) produce two identical EWPs with different central momentum due to the streaking of the IR field. Panel b) shows two EWPs at a time after the absorption in position space (green area). c) The momentum distribution of these EWPs is an interferogram (violet area) containing information about the amplitude and phase difference of the dipole transition matrix elements. d) The FFT of the momentum distribution consists of a DC central component and two AC components (blue line), which is spaced by $\left(\tau_{2}-\tau_{1}\right) p_{0}$ with respect to the DC component. After isolating the right $\mathrm{AC}$ (red dashed line), the phase difference can be recovered. 
The QSPIDER technique for amplitude and phase retrieval is useful only for a limited range of delays and relative shears between the copies, similar to the optical SPIDER $[20,21]$. These restrictions come from the conditions under which the retrieval algorithm can be applied.

Once the interferogram is available we can apply the QSPIDER retrieval algorithm which consists of five steps, namely:

(i) We compute the FFT of the interferogram, transforming from momentum space to a pseudo-position space. The resulting signal is depicted in Fig. 4.7(d). It consists of one central peak, the DC component, and two lateral signals, the AC components.

(ii) By placing a soft mask, the central DC part can be isolated to obtain the square amplitude of the EWP $\left|A\left(\mathbf{p}, \tau_{1}\right)\right|^{2}$. This simple procedure allows us to retrieve the amplitude of the EWP as if only one SAP was used.

(iii) To extract the phase difference $\Delta \phi_{21}$ it is necessary to use a soft mask (dotted red line in Fig. 4.7d)) to isolate the right AC term. For a better retrieval of this phase the DC and AC components need to be well separated. This can be controlled by "adjusting" the time delay $\tau_{\mathrm{x}}=\tau_{2}-\tau_{1}$ between the attosecond pulses, the central momentum of the EWP and the spectral width of the XUV pulse. For the attosecond pulses considered here, the best retrievals are obtained for delays around $\tau_{\mathrm{x}} \sim T_{0}$, i.e., the period of the laser pulse. Smaller delays $\tau_{\mathrm{x}}$ lead to an overlap between the AC and DC components, which are separated by $p_{0} \tau_{\mathbf{x}}$, thus ruining the retrieval algorithm. Attosecond pulses separated by one IR laser cycle have been obtained in HHG experiment with two colors $(\omega, 2 \omega)[147]$. Furthermore, the delay of the two attosecond pulses can be controlled by means of a segmented mirror [148]. After isolating the AC component, we compute the inverse FFT to obtain the phase difference $\Delta \phi_{21}$.

(iv) In analogy to the optical SPIDER technique, we can remove the known phases in Eq. (4.18) or Eq. (4.23) to extract the phase difference of the complex dipole transition matrix $\Delta \phi_{d}\left(\mathbf{p}, \tau_{1}, \tau_{2}\right)$. In principle, the phase difference can be obtained regardless of the value of the relative streaking between the two copies. Nevertheless, if the relative streaking $\Delta A_{\mathrm{L}}=A_{\mathrm{L}}\left(\tau_{2}\right)-A_{\mathrm{L}}\left(\tau_{1}\right)$ is sufficiently small, the phase difference gives the derivative of the phase

$$
\Delta \phi_{d}\left(\mathbf{p}, \tau_{1}, \tau_{2}\right) \approx \frac{\partial \phi_{d}}{\partial p} \Delta A_{\mathrm{L}}
$$

(v) Finally, an integration with respect to momentum yields the phase of the dipole transition matrix element. 
(i)

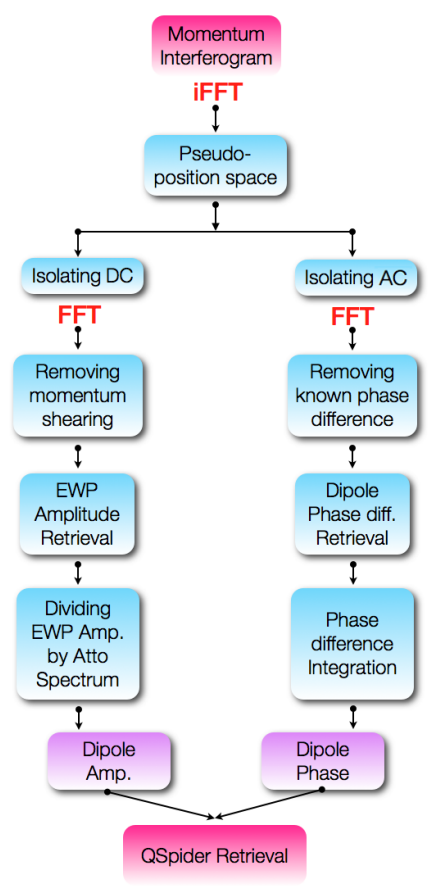

Figure 4.8: Basic QSPIDER algorithm. Schematic representation of the QSPIDER algorithm in analogy to the optical SPIDER algorithm.

The optical SPIDER technique is not able to retrieve the linear spectral phase term which determines the absolute timing of the pulse, and neither the absolute phase [21].

In QSPIDER, the situation is somewhat different: the linear term of the momentumdependent dipole phase can be retrieved since all other contributions in Eq. (4.18) can be computed. Nevertheless, the absolute dipole phase cannot be retrieved. Therefore, when integrating the retrieved phase derivative to obtain $\phi_{d}$, an arbitrary integration constant may be added. The removal of the Volkov phase

$$
\frac{1}{2} \int_{\tau_{1}}^{\tau_{2}} d t^{\prime \prime}\left(\mathbf{p}+\mathbf{A}_{\mathrm{L}}\left(t^{\prime \prime}\right)\right)^{2}=\frac{p^{2}}{2} \tau_{\mathrm{x}}+\mathbf{p} \cdot \int_{\tau_{1}}^{\tau_{2}} \mathbf{A}_{\mathrm{L}}\left(t^{\prime \prime}\right) d t^{\prime \prime}+\frac{1}{2} \int_{\tau_{1}}^{\tau_{2}} \mathbf{A}_{\mathrm{L}}^{2}\left(t^{\prime \prime}\right) d t^{\prime \prime}
$$

is straightforward if a full characterization of the IR laser pulse is available. The same holds for the Coulomb-corrected phase of Eq. (4.22). The time delay $\tau_{\mathrm{x}}$ can be calibrated by measuring the spacing between fringes in the case of ionization by two attosecond pulses without an IR field. The relative shear between the two copies can be measured by blocking alternatively one of the attosecond pulses while leaving the IR laser pulse active and this would result in the momentum distribution streaked by the value of the vector potential $A_{\mathrm{L}}\left(\tau_{1}\right)$ and $A_{\mathrm{L}}\left(\tau_{2}\right)$ alternatively. 
The spectral phase of the XUV pulse $\Delta \phi_{\mathrm{X}}$ must be characterized either by some other technique available $[62,58]$ or by an estimation of the intrinsic chirp based on the HHG process producing the APT. When the XUV and IR pulses are known, we can also evaluate the contribution $\Delta \phi_{\text {LIC }}$.

So far we have fully described the QSPIDER schemes fundamental elements and the retrieval algorithm as well. The next Sections will be devoted to test the QSPIDER technique to retrieve the dipole amplitude and the dipole phase for different atomic potentials.

First, we will use the SFA formalism described in Section 4.2.3 to compute the final momentum interferogram for the QSPIDER technique utilizing as a test system the $\mathrm{He}^{+}$. Second, we will employ the full TDSE calculations to characterize a complex EWP and from this the complex dipole transition matrix element (for the cases of the longand short-range potentials). Assuming a small collection angle, we can restrict ourselves to a reduced 1D model, i.e., we focus on electrons emitted along the polarization axis. This is possible by considering the laser is linearly polarized. In case of the SFA, this means we will take a line of the momentum distribution along the $z$-axis for our numerical calculations for the QSPIDER conditions.

\subsubsection{Results within Strong Field Approximation}

In order to test the QSPIDER within the SFA, we use the ground state $\left|\phi_{1 s}\right\rangle$ of the helium ion with the $I_{p}=2.0$ a.u., and the first excited state $\left|\phi_{2 s}\right\rangle$ with $I_{p}=0.5$ a.u. $(13.60 \mathrm{eV})$.

For the first case, the dipole has a constant phase (see Fig. 4.9a)) due to the plane waves $\Psi_{\mathbf{p}}(\mathbf{r})=\frac{1}{(2 \pi)^{3 / 2}} e^{i \mathbf{p} \cdot \mathbf{r}}$ used in the SFA matrix elements and the symmetry of the initial state. Eq. (4.3) shows the analytical formula for this dipole transition matrix element. This dipole phase helps us to distinguish extra phases occurring in the measurement or in the retrieval algorithm.

In the second case, the dipole phase is constant except for a phase jump of $\pi$ at a momentum where the amplitude of the dipole is zero. The analytical form of the dipole transition matrix element, $d_{z, 2 s}(\mathbf{p})=-\left\langle\mathbf{p}|z| \psi_{2 s}\right\rangle$, for the transition $2 s$-continuum reads:

$$
d_{z, 2 s}(\mathbf{p})=-i \frac{2^{9 / 2} \gamma^{5 / 2}}{\pi} \frac{2 \gamma^{2}-\mathbf{p}^{2}}{\left(\gamma^{2}+\mathbf{p}^{2}\right)^{4}} p_{z}
$$

where $\gamma^{2}=2 I_{p}$. Fig. 4.9 shows a cut of the bound-free dipole transition matrix element 
for the $1 s$ and $2 s$ states of the helium ion. The cut is done along the $p_{z}$-axis and the analytical expression for the $2 s$ dipole is calculated as in reference [140].

a)

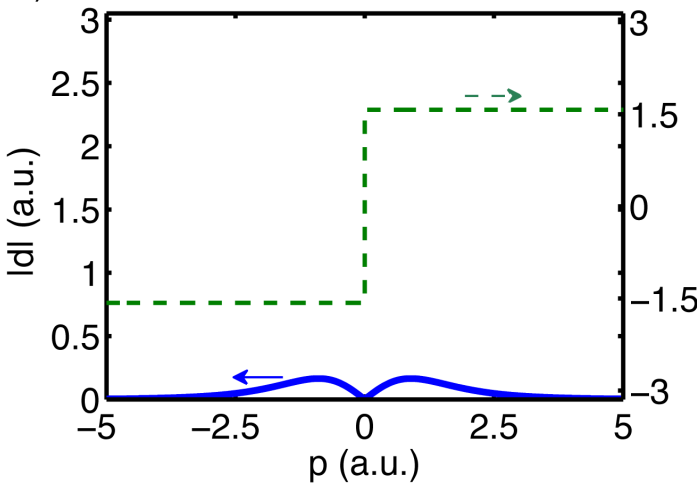

b)

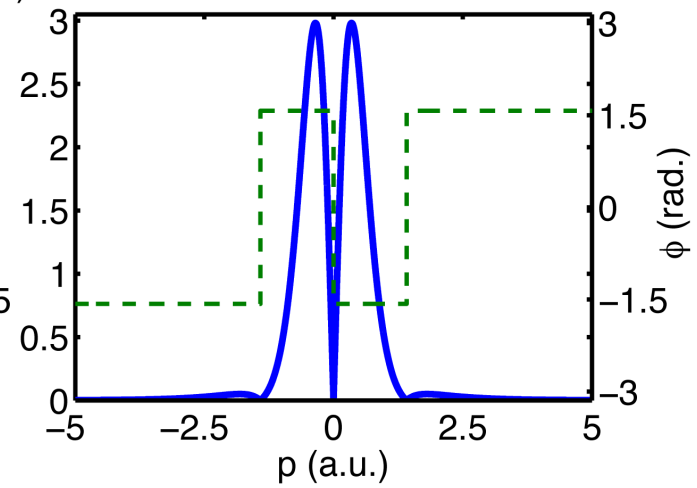

Figure 4.9: Complex dipole structure for the $1 s$ and $2 s$ of $\mathbf{H e}^{+}$. In the panels a) and b) we depict a cut along $z$-axis of the amplitude (blue line) and phase (green dashed line) of the bound-free dipole transition matrix element from the $1 s$ and $2 s$ orbitals to the continuum. These continuum states are PWs. The complex dipoles are analytical expressions calculated from [140].

Note that the dipole amplitude in case of Fig. 4.9b) is larger compared to the amplitude in a). In addition, a different structure is also obtained in case of a $2 s$ dipole. This is so, due to the different shape of the orbital $2 s$ compared to the $1 s$. In Fig. $4.9 \mathrm{~b}$ ), the node at a position about $p=1.4$ a.u., gives us information about the structure of the orbital $2 s$.

\section{Characterization of the complex dipole for the $1 \mathrm{~s}$}

In the case of $\left|\phi_{1 s}\right\rangle$ state we have used the following pulse parameters to compute our QSPIDER interferogram. The APT consists of two pulses with central frequency $\omega_{\mathrm{X}}=2.845$ a.u. $(77.4 \mathrm{eV})$. The pulse envelope is a gaussian for both pulses with FWHM $\sim 170$ as. The intensity of the XUV pulses is $I_{\mathrm{X}}=10^{13} \mathrm{~W} / \mathrm{cm}^{2}$. On the other hand, the parameters of the IR pulse used here are: wavelength $\lambda_{0}=800 \mathrm{~nm}$ $\left(\omega_{0}=0.057\right.$ a.u. $)$ and intensity $I_{0}=5.0 \times 10^{11} \mathrm{~W} / \mathrm{cm}^{2}$. The time duration of the laser pulse is around FWHM $\sim 4$ fs and the envelope of the field is a gaussian function. The momentum grid parameters are $\Delta p=0.001$ a.u., and the momentum maximum value $L_{p}=12.5$ a.u.

We numerically integrate Eq. (2.95) by using a fourth-order Runge-Kutta numerical method [103] for the above described pulse parameters and scanning the time delay 
$\tau_{\mathrm{x}}=\tau_{2}-\tau_{1}$ between the XUV attosecond pulses. We have fixed the delay $\tau_{1}$ between the first XUV and the IR laser (see Fig. 4.10a)). Then, the calculated final momentum interferograms, $\mathrm{S}\left(p, \tau_{\mathbf{x}}\right)$, are scanned on the time-delay $\tau_{\mathbf{x}}$. That is equivalent to vary the time delay $\tau_{2}$ between the second attosecond pulse and the IR. A very good illustration about this delays, is depicted in Fig. 4.10a).

Fig. 4.10 shows the final momentum interferogram for QSPIDER as a function of the time delay $\tau_{\mathrm{x}}$. The range time delay $\tau_{\mathrm{x}}$ is around one cycle of the IR laser field.

Note that this momentum interferogram shows an interference pattern that is dependent on the time delay $\tau_{\mathrm{x}}$. It is important to mention that we can associate a momentum relative shearing to each time delay $\tau_{\mathrm{x}}$ given by $\Delta A_{\mathrm{L}}=A_{\mathrm{L}}\left(\tau_{2}\right)-A_{\mathrm{L}}\left(\tau_{1}\right)$.

Once the final interferogram is obtained we have employed the QSPIDER algorithm to retrieve, first, the EWP amplitude and phase derivative and second, the amplitude and the phase of the dipole transition matrix element. This dipole characterization is done within the spectral bandwidth of the attosecond pulse or EWP.

We have applied the QSPIDER algorithm to the interferogram (see Fig. 4.10) at each time delay $\tau_{\mathrm{x}}$. The best delay for the retrieval is close to one cycle of the IR and the momentum shear $\Delta A_{\mathrm{L}}=0.04$ a.u.

Figures 4.11b) and c) show the retrieved momentum distribution of the EWP for negative and positive momenta compared to the case of the absorption from a single attosecond pulse without IR field, respectively. In addition, we depict the retrieved derivative of the dipole phase. The results show a very good agreement in the region defined by the spectral momentum width of the EWP. Figures 4.11d-e) show the retrieval of the dipole amplitude and the dipole phase, respectively. The amplitude of the dipole can be found by dividing the retrieved amplitude of the EWP by the XUV amplitude $\left|E_{0, \mathrm{X}}\left(\omega_{\mathbf{p}}\right)\right|$ [139]. The agreement between the retrieved amplitude and the exact one is very good over all the accessible range. The retrieved dipole phase after integration is constant which indicates that the algorithm does not add any artificial phase.

\section{Characterization of the complex dipole for the $2 s$}

In the second case, we analyze the first excited state $\left|\phi_{2 s}\right\rangle$. The bound-free dipole transition matrix element has a node around $p_{z}=1.4$ a.u. and the phase exhibits a phase jump by $\pi$ at this final momentum (see Fig. 4.9b)). This behavior is similar to that found around the Cooper minimum in photoionization of Argon [149]. The APT consists of two pulses with $\omega_{\mathrm{X}}=1.605$ a.u. $(43.7 \mathrm{eV})$ and FWHM $\sim 215$ as. The IR field parameters are the same as in the first example.

The final momentum interferogram is also calculated as a function of the time delay $\tau_{\mathrm{x}}$. 

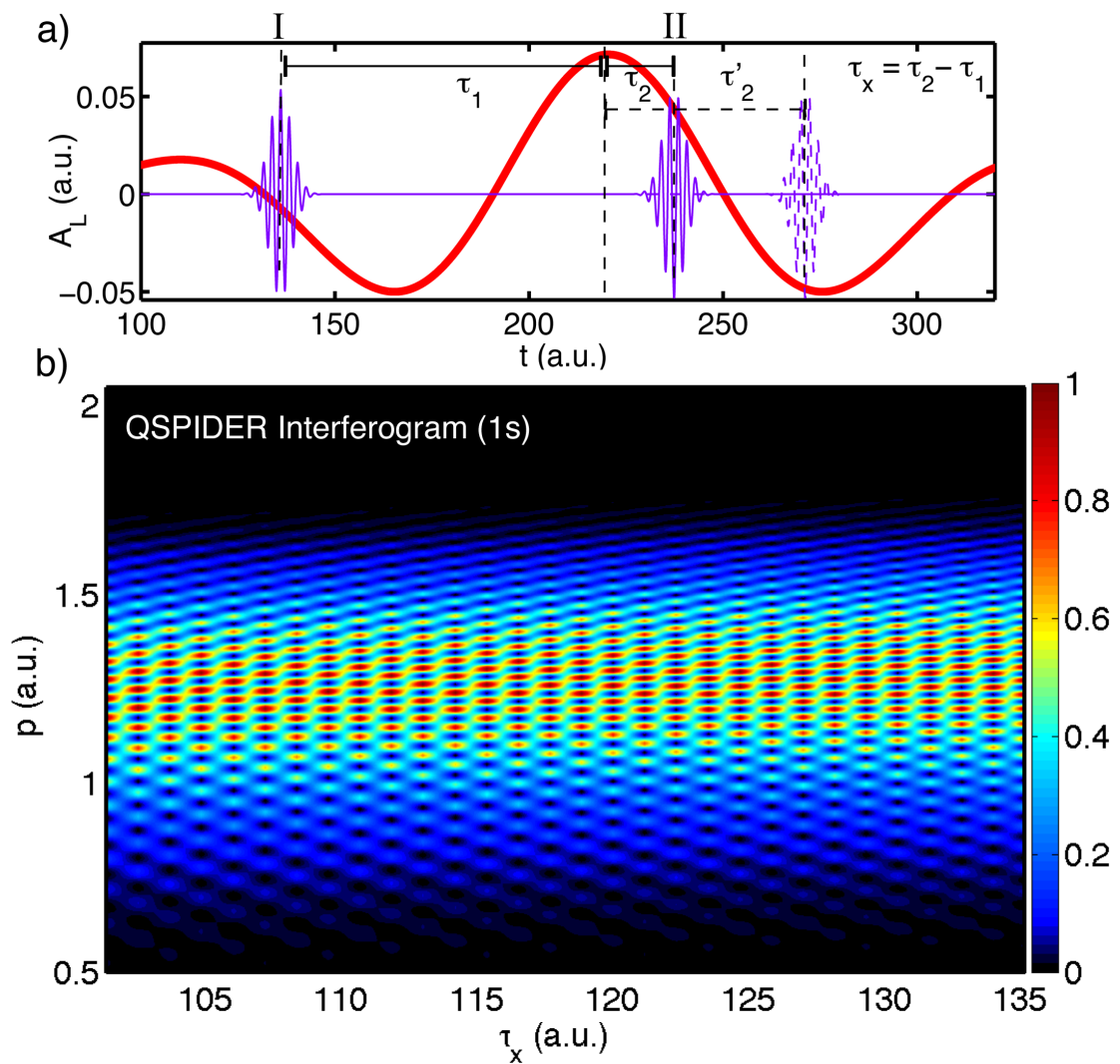

Figure 4.10: Streaked interferogram in case of $1 s$ of $\mathbf{H e}^{+}$: SFA model. The final momentum interferogram (color scale in arbitrary units) as a function of the time delay $\tau_{\mathrm{x}}$ between the XUV pulses is depicted in $\mathrm{b}$ ). The used pulses are two colors: The first color is the IR laser field. Its vector potential $A_{\mathrm{L}}(t)$ is depicted by the solid red line of the plot a). The second color is the train of two attosecond pulses (solid violet line) delayed between them by $\tau_{\mathrm{x}}$. The time delay $\tau_{1}$ between the first (I) attosecond and the vector potential is fixed while the time delay of the second (II) attosecond $\tau_{2}$ is varied. The time delay $\tau_{2}$ is changed until $\tau_{2}^{\prime}$ which denotes the final time delay of the second attosecond pulse. This pulse is depicted in dashed violet line. 

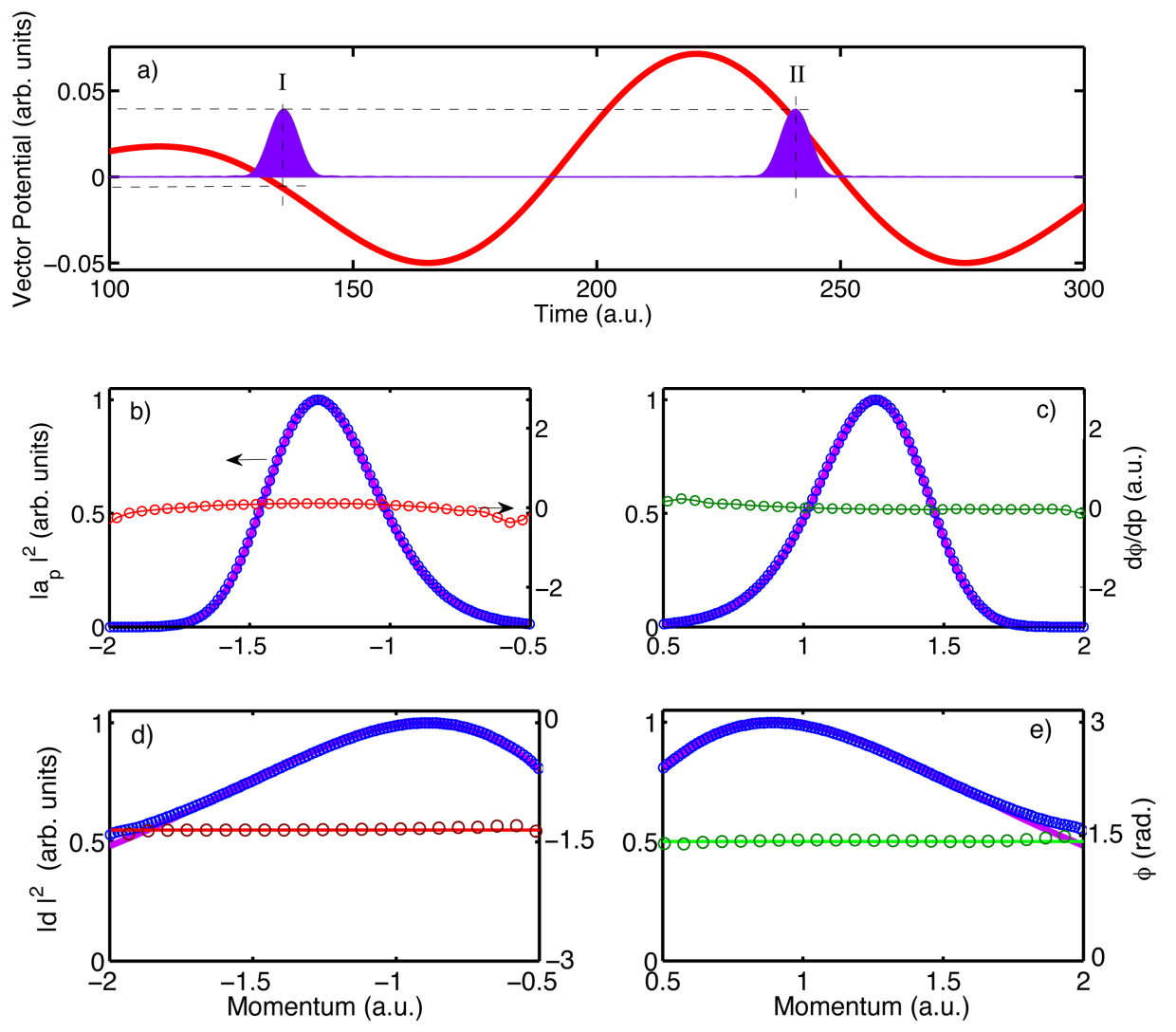

Figure 4.11: QSPIDER retrieval for the $1 s$ of $\mathbf{H e}^{+}$: SFA model. a) The red solid line shows the vector potential of the IR laser pulse. The violet area is the field envelope of the APT. The retrieved momentum distribution of the EWP (blue circles) compared to the exact momentum distribution (violet solid line) from the interaction with an SAP in absence of the IR field is plotted in b) and c) for negative and positive momenta, respectively. The retrieved derivative of the dipole phase is shown in red and green circles respectively in b) and c). The retrieved squared amplitude (blue circles) and phase (red and green circles) of the dipole matrix element is compared to the exact squared amplitude (violet solid line) and phase (red and green solid line) in d) and e). 
The results are shown in Fig. 4.12. The time delay range $\tau_{\mathrm{x}}$ can be understood easily by analyzing Fig. 4.12a). Similar to the dipole characterization for the $\left|\phi_{1 s}\right\rangle$ state, we have fixed the first attosecond pulse (I) and varied the time delay $\tau_{2}$ of the second one. The corresponding range to the time delay $\tau_{\mathrm{x}}=\tau_{2}-\tau_{1}$ between the XUV pulses is $\tau_{\mathrm{x}}=T_{0} / 2$ and $1.4 T_{0}$.

Fig. 4.12b) clearly shows that while the fixed first EWP does not change its final momentum, the vector potential of the IR streaks the second emitted EWP when the second XUV pulse changes its relative time delay $\tau_{2}$. Note, that this streaked delaymomentum interferogram encodes also information about the IR laser field.

In addition, we find that this momentum interferogram has a clear signature of the structural dipole node. Note that we are referring to the zero in the EWPs interferogram as a function of the time delay $\tau_{\mathrm{x}}$. This zero is clearly streaked by IR vector potential $A_{\mathrm{L}}(t)$ at the time delay $\tau_{2}$. We are not referring to the zero set by one interferogram at single delay $\tau_{\mathrm{x}}$.

We have employed the QSPIDER algorithm to each time delay $\tau_{\mathrm{x}}$ to reconstruct the EWP amplitude and phase difference. The results are shown in Fig. 4.13 a) and b). It is noted that the EWP is streaked exactly by the negative value of the vector potential $-A_{\mathrm{L}}\left(\tau_{2}\right)$ at the time delay $\tau_{2}$. On the other hand, we can see that the EWP phase difference (or dipole phase difference) $\Delta \phi_{d}\left(p, \tau_{\mathrm{x}}\right)$ is hardly changing from $-\pi$ to $\pi$ with respect to the time delay axis. This phase difference is similar to the Dirac delta distribution about $\tau_{\mathrm{x}} \sim T_{0}$. The change of the sign in $\Delta \phi_{d}\left(p, \tau_{\mathrm{x}}\right)$ is due to the change momentum shearing $\Delta A_{\mathrm{L}}$, which depends on $\tau_{\mathrm{x}}$.

Note that, this retrieved phase difference is exactly that one we can expect from the phase difference of the analytical dipole phase depicted in Fig. 4.9b).

After removing the extra momentum shift induced by the IR vector potential and to integrate the phase difference of Fig. 4.13, we select the best time delay $\tau_{\mathrm{x}}$ or momentum shearing retrieval and show the results in Fig. 4.14. The plots 4.14b-c) depict the retrieved momentum distribution of one EWP compared to the case of the absorption from a single attosecond pulse without IR field. We can also observe that the retrieved derivative of the phase is in very good agreement with the exact phase derivative. Figures $4.14 \mathrm{~d}-\mathrm{e})$ show the phase after the integration with the correct jump around $p_{z}=1.4$ a.u., as expected. The agreement between the retrieved and exact dipole amplitudes is very good over all the accessible range and for negative and positive momentum.

In conclusion, the results under SFA model, shows that the QSPIDER technique is able to fully characterize the EWP and the complex bound-free dipole transition 

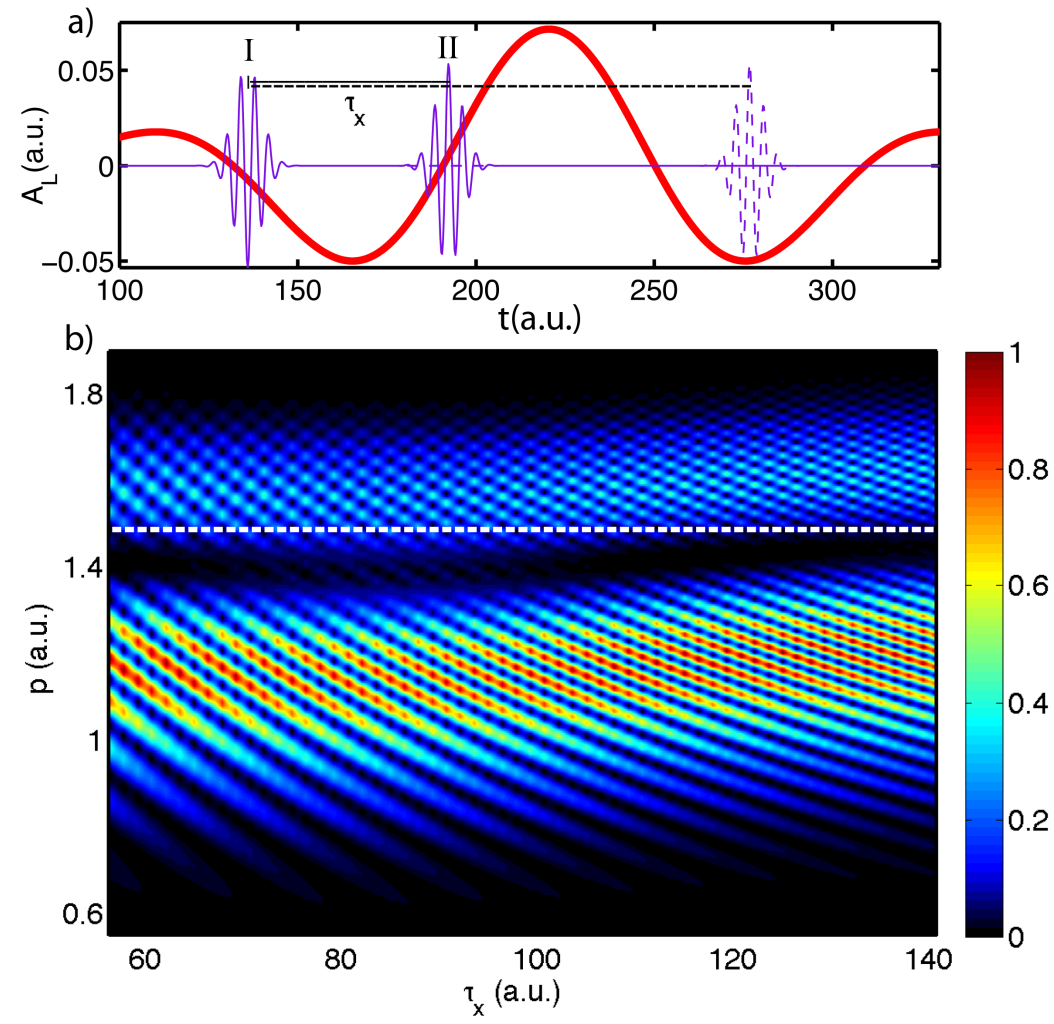

Figure 4.12: QSPIDER momentum interferogram for the $2 s$ of $\mathrm{He}^{+}$: SFA model. a) The APT and the vector potential are depicted in red line and violet solid and dashed line, respectively. The solid violet line denotes the first APT which has a time delay between them about $\tau_{\mathrm{x}}=T_{0} / 2$. The dashed violet line denotes the second XUV at a different and final time delay $\tau_{\mathrm{x}}=1.1 T_{0}$. In the plot $\mathrm{b}$ ) we show the final streaked momentum interferogram (color scale) as a function of the time delay $\tau_{\mathrm{x}}$ between each attosecond pulse. 

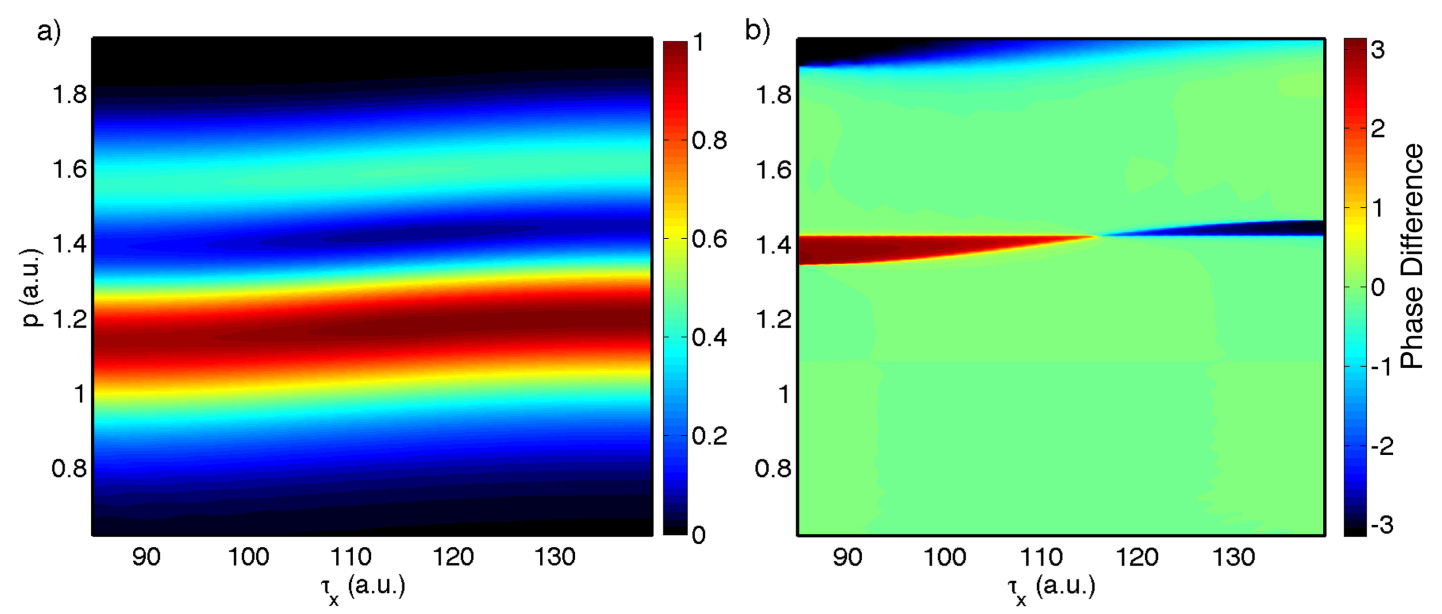

Figure 4.13: QSPIDER for the 2s of $\mathbf{H e}^{+}$: SFA model. QSPIDER retrieval of the momentum EWP amplitude a) and phase difference b) (dipole phase) as a function of the time delay $\tau_{\mathrm{x}}$ between each of the attosecond pulses.

matrix element in an atom, ion or molecule undergoing photoionization. The technique requires interaction of the system with a train of two attosecond pulses in the presence of a streaking IR field. QSPIDER can retrieve amplitudes and phases faithfully in a region defined by the central frequency and spectral width of the attosecond pulses. If the pulses are well known and the Coulomb-laser coupling is taken into account, the technique measures the complex bound-free dipole transition matrix element, which can be used in the future to access structural information of larger systems.

\subsubsection{Results within the Time Dependent Schrödinger Equation}

In the last Section, we have shown the results of the QSPIDER by employing the SFA. A more realistic description of the phases of the system can be obtained by using the numerical solution of the TDSE. Such calculations include all the laser system couplings exactly.

\section{Definition of the systems}

The 1D time independent Hamiltonian $H$, that describes the interaction of the electron with the atomic system nucleus, reads:

$$
H=\frac{1}{2} p_{z}^{2}+V_{0}(z)
$$



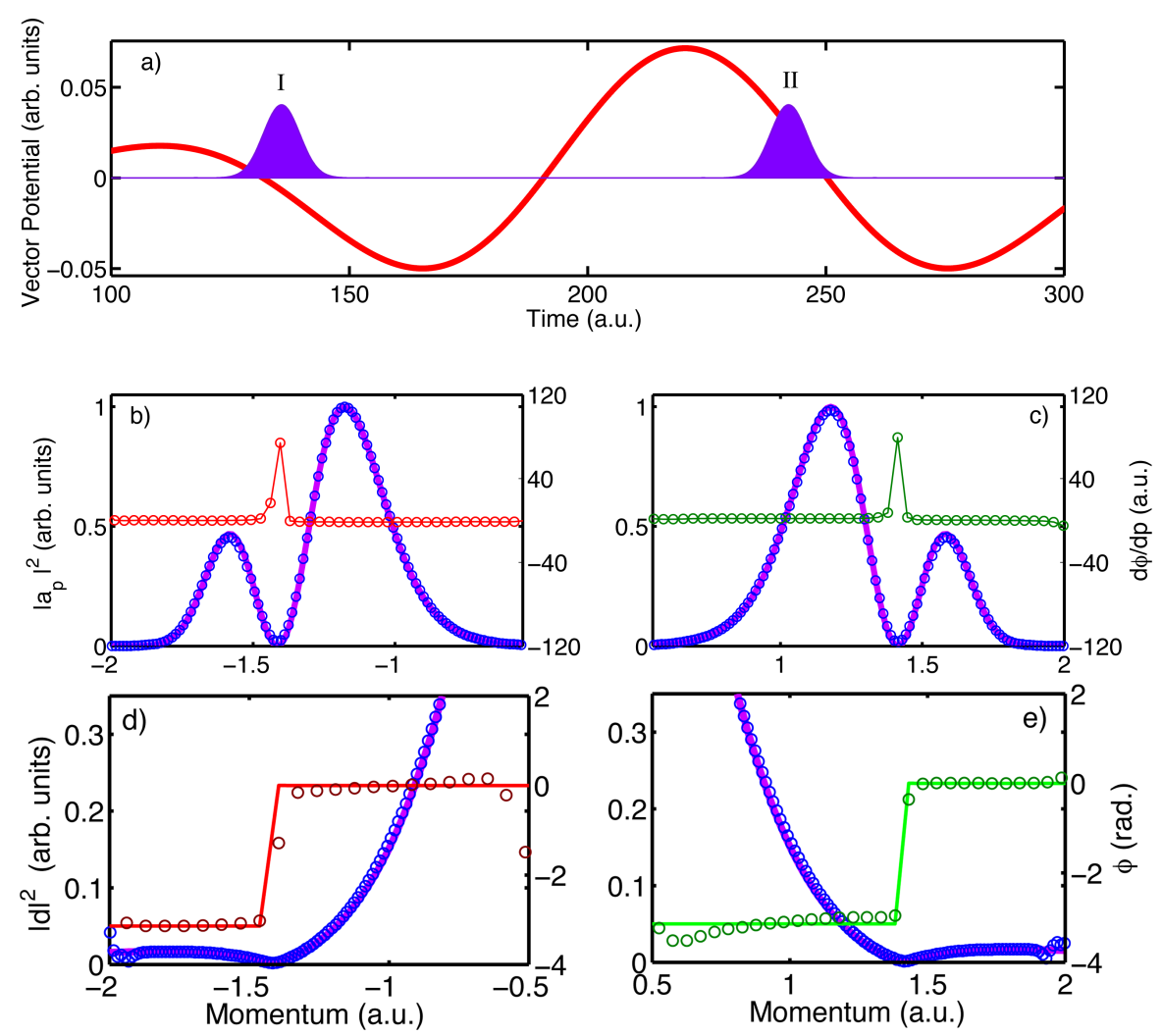

Figure 4.14: QSPIDER for the $2 s$ of the $\mathbf{H e}^{+}$: SFA model. Lines and symbols as in Fig. 4.11. (a) Laser pulses used in our simulation. (b-c) Retrieval of the amplitude of the EWP (blue circles) versus the exact amplitude of the EWP (violet solid line) and retrieval of the derivative of the dipole phase (red and green circles). (d-e) Retrieval of the dipole amplitude (blue circles) and dipole phase (red and green circles) of the EWP. The QSPIDER retrieval (red and green circles) versus the analytical (red and green solid line) dipole phase are shown for negative and positive momentum, respectively. 
where, $p_{z}=-i \frac{\partial}{\partial z}$, is the momentum operator and $V_{0}(z)$, is the potential of the system. We choose two 1D potentials to solve TISE and TDSE. The short-range Yukawa potential is:

$$
V_{0}(z)=-\frac{Z}{\sqrt{a+z^{2}}} \exp -\left(\sqrt{a+z^{2}} / a_{s}\right)
$$

where, $a$, is a soft-core parameter and, $a_{s}$, is the screening charge length of the potential. On the other hand, we employ a long-range Coulomb potential similar to the one used in Section 4.2.1.

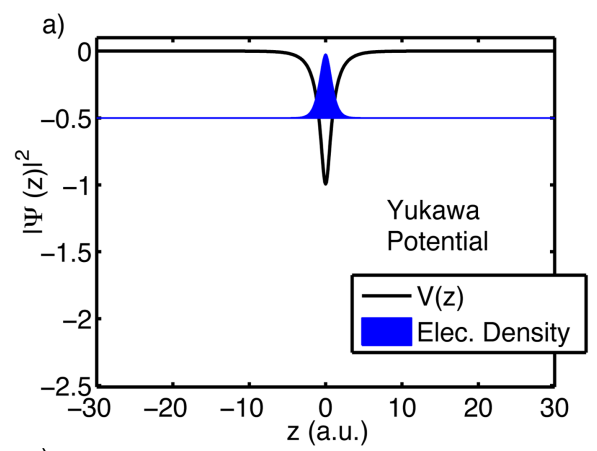

c)
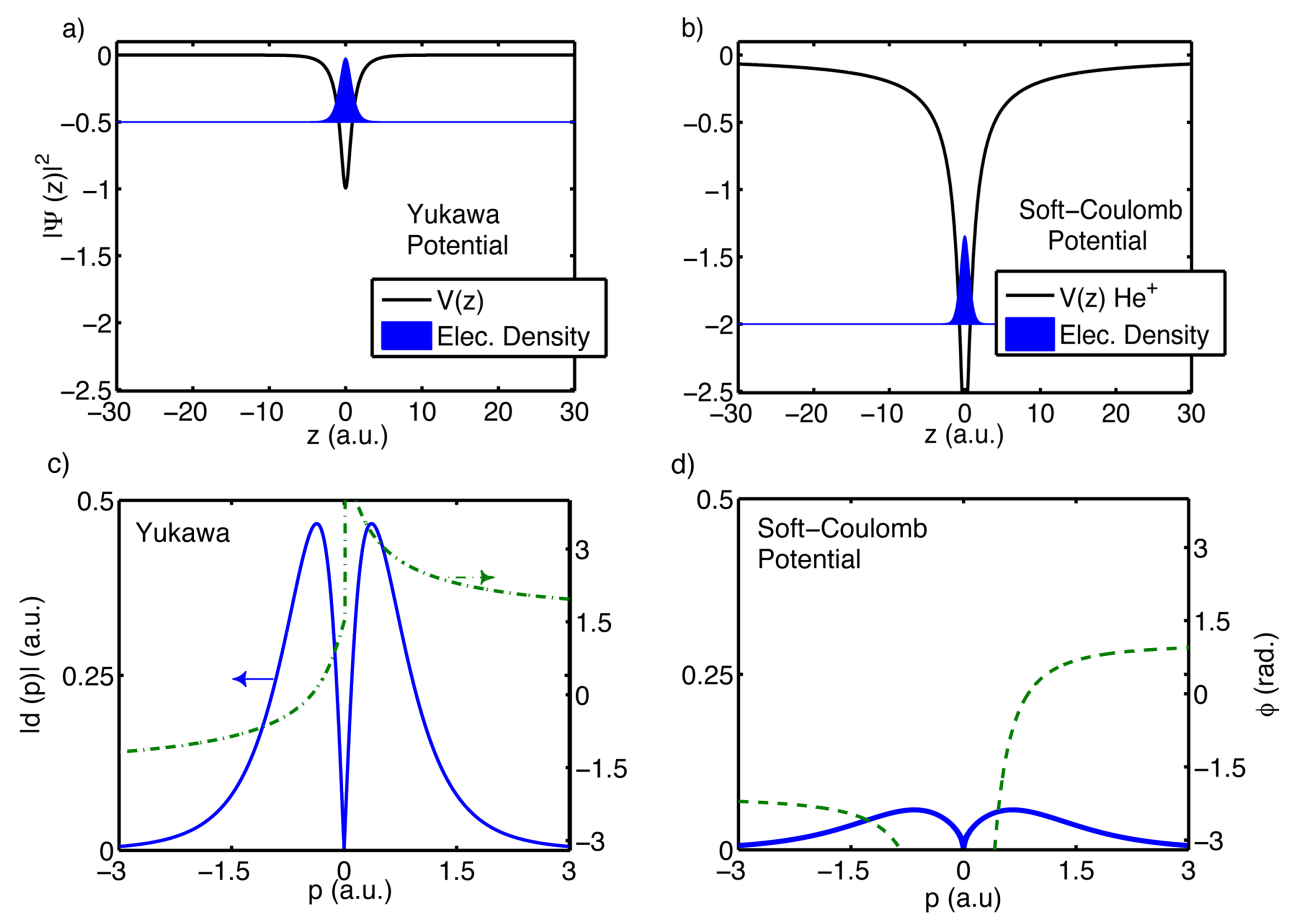

Figure 4.15: 1D Potentials, bound states, and complex dipoles. Figures (a) and (b) show the 1D shape potentials (black solid line) and the electron density (blue area) for the ground states in case of Yukawa potential (a) and $\mathrm{He}^{+}$(b), respectively. The dipole amplitude $|d(p)|$ (blue solid line) and dipole phase $\phi_{d}(p)$ (green dashed line) are depicted for the boundfree transition of the both cases; Yukawa potential in panel (c) and soft-core Coulomb potential in (d), respectively. The dipoles were calculated by projecting on scattering waves $\Psi_{p}(z)$ of momentum $p$ corresponding to the short-range Yukawa and long-range Coulomb potentials, respectively.

We have taken the soft-core parameters in such a way that they match, on one hand, the ionization potential energy of the ground state, in case of Yukawa potential 
$(Z=1), I_{p}=0.5$ a.u., and, on the other hand, the ionization potential energy, $I_{p}=2.0$ a.u., of the helium ion, $(Z=2$, Coulombic potential). The bound states are calculated via imaginary time propagation with a step of $\Delta t=-i \times 10^{-4}$ a.u., for each case. The grid parameters are $\Delta z=0.01$ a.u., and its whole length $L_{z}=2400$ a.u.

Figs. 4.15a-b) depict the Yukawa and soft-core Coulomb potentials with their respective ground states. Furthermore, we have calculated the exact complex dipole $d(p)=-\left\langle\psi_{p}|z| \psi_{0}\right\rangle$ that will be characterized by using the QSPIDER technique.

The dipoles were numerically computed by projecting on the 1D scattering continuum waves $\Psi_{p}(z)$, which will be described in the numerical methods Section.

Figs. 4.15c-d) show the dipole amplitudes and dipole phases in the case of Yukawa and Coulomb potentials, respectively. Note that the dipole phase depicted in the case of $\mathrm{He}^{+}$is the so-called short-range phase or phase-shift of the scattering waves for this system. These exact dipoles will allow us to compare them to the final amplitude and phase characterized via the QSPIDER technique.

\section{Short-range Yukawa potential}

Here we present the results of the QSPIDER technique to extract the dipole amplitude and phase by using the full numerical integration of the TDSE for the case of a shortrange potential well.

The TDSE momentum distributions are calculated after the end of the APT and IR pulse. To this end, the final TDSE wavefunction is projected onto the continuum scattering waves. The potential well parameters used in our simulation were described above and the momentum spacing used for the momentum distribution is $\Delta p=0.001$ a.u.

The APT consists of two pulses with $\omega_{\mathrm{X}}=2.0$ a.u. $(54.4 \mathrm{eV})$ and FWHM $\sim 243$ as and the IR field parameters are the same as in the first example, but now the envelope is a sine squared function. The delay for the QSPIDER retrieval is chosen close to one IR period, as shown in Fig. 4.16a). The momentum shear is $\Delta A_{\mathrm{L}}=-0.04$ a.u., i.e., much bigger than the momentum spacing $\Delta p$. Figures $4.16 \mathrm{~b}-\mathrm{c}$ ) show the excellent agreement between the retrieved EWP momentum distribution and the case of the absorption from a single attosecond pulse without IR field. The retrieved derivative of the phase is in very good agreement with the exact phase derivative within the EWP momentum bandwidth. Note that the phase derivative would be equal to zero if PWs were used in the dipole transition matrix elements. Thus, our results show that QSPIDER retrieves the linear term in the exact dipole phase quite accurately.

As a general rule, the shear $\Delta A_{\mathrm{L}}$ should not be chosen too small, as it was found in the optical SPIDER technique [150,151]. Otherwise small errors in the measured phase 

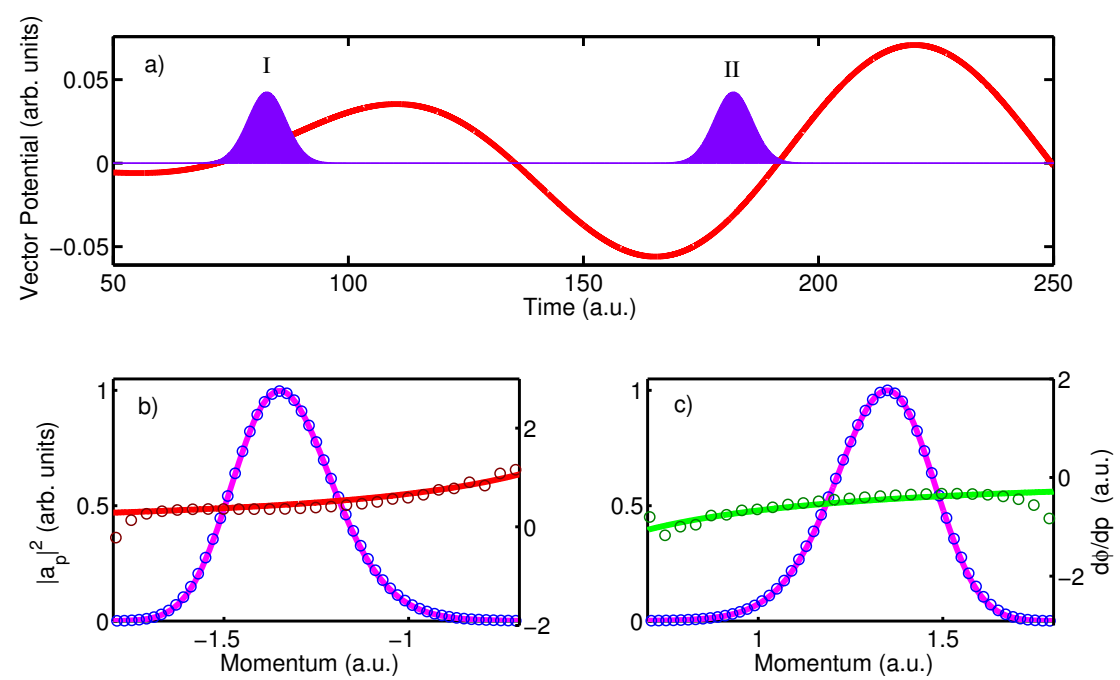

Figure 4.16: QSPIDER for the ground state of a Yukawa potential: 1D TDSE model. The IR vector potential (red line) and the APT envelopes (violet area) are depicted in a). The time delay between the XUV attosecond pulses is $\tau_{\mathrm{x}} \sim T_{0}$. The negative (b) and positive momenta (c) of the retrieved EWP amplitude (blue circles) and the corresponding derivative of the dipole phase are shown in b). The red and the green circles denote the retrieved derivative of the dipole phase. The exact EWP amplitude is depicted in violet solid line. Also, the exact momentum derivatives of the dipole phase for negative and positive momenta are in red and green solid lines, respectively. 
difference $\Delta \phi_{d}$ will lead to large errors in the retrieved phase derivative $\Delta \phi_{d} / \Delta A_{\mathrm{L}}$. An estimation of the required shear can be obtained by measuring first the residual phase difference $\Delta \phi_{d}^{(0)}$ for vanishing shear in the presence of the IR laser field. The shear for QSPIDER should then be chosen such that the measured $\Delta \phi_{d}$ is much larger than $\Delta \phi_{d}^{(0)}$.

\section{Long-range potential}

In this Section, we consider the ground state of a model helium ion described by the soft-core Coulombic potential which was defined in Section 4.2.1.

The photoelectron momentum distribution is obtained from the numerical solution of the TDSE by projection on the exact scattering continuum waves. Due to the long-range character of the potential, we require the continuum waves to satisfy the boundary condition of Eq. (4.19).

The simulation parameters are $\Delta z=0.01$ a.u., and $N_{z}=250000$ grid points. The momentum grid step is $\Delta p=0.001$ and the length $L_{p}=10$ a.u. The attosecond pulse shape is gaussian for both XUV pulses with FWHM $\sim 170$ as and central frequency $\omega_{\mathrm{X}}=4.0$ a.u. Figures $4.17(\mathrm{~b}-\mathrm{c})$ show the results.

The retrieved derivative of the phase is in good agreement with the exact phase derivative within the EWP momentum bandwidth. In this case both the LIC phase and the Coulomb laser coupling phase are important and contribute to the overall phase in similar weights. Therefore it is necessary to calculate these contributions with high precision. Further, as the derivative of the phase is calculated by dividing two quantities, for small values of relative shear $\Delta A_{\mathrm{L}}$ we need a great precision for these two phases which are the most sensitive of all the quantities considered here.

In conclusion, our numerical results of the QSPIDER within SFA and the reduced 1D-TDSE model demonstrated clearly that this quantum interferometry technique works reasonably well to extract the complex EWP and the complex dipole transition matrix element. The QSPIDER utilizes an APT of two pulses in presence of a weak IR-laser field which results in a final EWPs interferogram. This allows us to extract the complex dipole transition matrix element within the spectral bandwidth range defined by one of the XUV attosecond pulse copies. It is important to point out that QSPIDER inherits all the issues of the optical SPIDER to extract the phase, e.g., a detector with high spectral resolution is needed in order to recover a very accurate phase derivative.

We will extend QSPIDER to characterize the EWP and dipoles plane and not only along the polarization laser axis. The next Section will be studied how to retrieve the 

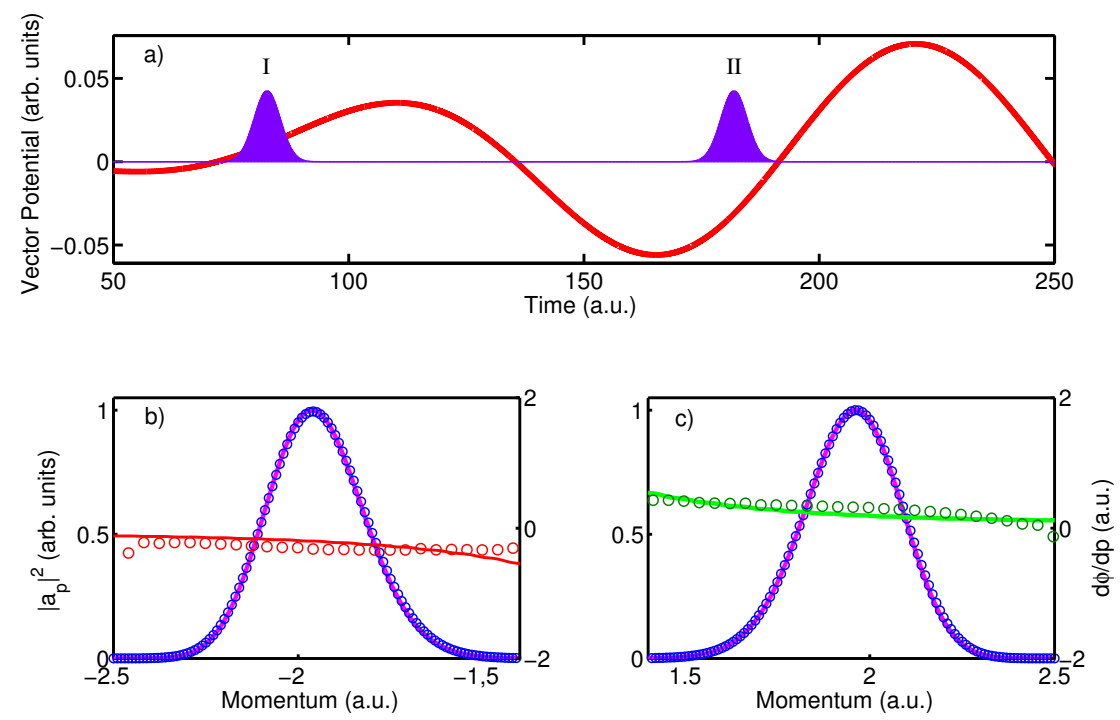

Figure 4.17: QSPIDER for the ground state of a Coulomb potential: 1D TDSE model. The IR vector potential (red line) and the APT envelopes (violet area) are depicted in a). The time delay between the XUV attosecond pulses is $\tau_{\mathrm{x}} \sim T_{0}$. The negative (blue circles) (b) and positive momenta (c) of the retrieved EWP amplitude and the corresponding derivative of the dipole phase for negative (red circles) and positive (green circles) momenta are shown in b). The retrieval results are compared to the exact calculation of the EWP amplitude (magenta solid line) and phase: red solid and green solid lines in case of negative and positive momenta, respectively. 
dipole or EWP phase in a 2D momentum plane.

\subsection{Lateral momentum-shearing EWP interferometry tech- nique}

In the last Section we have shown that the QSPIDER technique works to retrieve the EWP amplitude and the structural complex dipole transition matrix element within the spectral region defined by the XUV attosecond pulses. However, this implementation restricts the motion of the electron wave packet along a $1 \mathrm{D}$ line, i.e., we lost the angular information.

We remind that in the lab a momentum interferogram can be measured in a plane $\left(p_{x}, p_{y}\right)$ by a velocity map imagining technique. This allows us to assume that an analogy between the optical lateral shearing interferometry technique to extract the wavefront of the laser beam can be employed to EWPs interferometry too.

The general purpose of the next Sections is to extend our characterization of the complex EWPs and complex dipoles to a momentum plane $\left(p_{x}, p_{y}\right)$. To this end, we will take advantage of the analogy between the optical wavefront characterization and the quantum determination of the EWP amplitude and phase.

There is a large variety of optical wavefront techniques, i.e., Hartmann-Shack [131], lateral shearing interferometry [130], Foucault knife-edge test, [132], etc. The lateral shearing is one of the most simple implementations to reconstruct the laser beam wavefront. This technique is similar to the optical SPIDER, but here the shearing is induced in the spatial wavefront of the laser beam and not in the spectral domain. To characterize the space phase profile [152], two partial derivatives need to be implemented in the measurement process: one on the $x$ and another one on the $y$ direction. Here we assume that the laser is propagating along the $z$-axis. Fig. 4.18 depicts a general scheme about the lateral shearing interferometry technique.

The upper panel a) shows the two copies of the beam, $\mathrm{A}_{F, 1}(x, y+\Delta y / 2)$ and $\mathrm{A}_{\mathrm{F}, 2}(x, y-\Delta y / 2)$, depicted by two different color circles. The copies are lateral shifted one with respect to the other one along the $y$-axis by an amount of $\Delta y$. The lower panel a) shows the same, but the lateral shearing is now along the $x$-direction.

The two lateral shearings, create two different interferograms in the overlap regions which contain full information about the phase differences along the $x$ and $y$-directions. These interferograms are depicted in the upper (sheared on $y$ ) and lower (sheared on $x$ ) panel of Fig. 4.18b). If the lateral shearings, $\Delta x$ and $\Delta y$, are small enough, i.e., around of the grid spacing order, the phase differences, $\Delta \Phi_{y}(x, y)$ and $\Delta \Phi_{x}(x, y)$, can 
a)
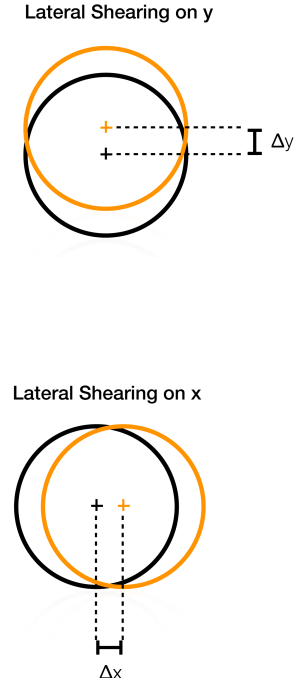
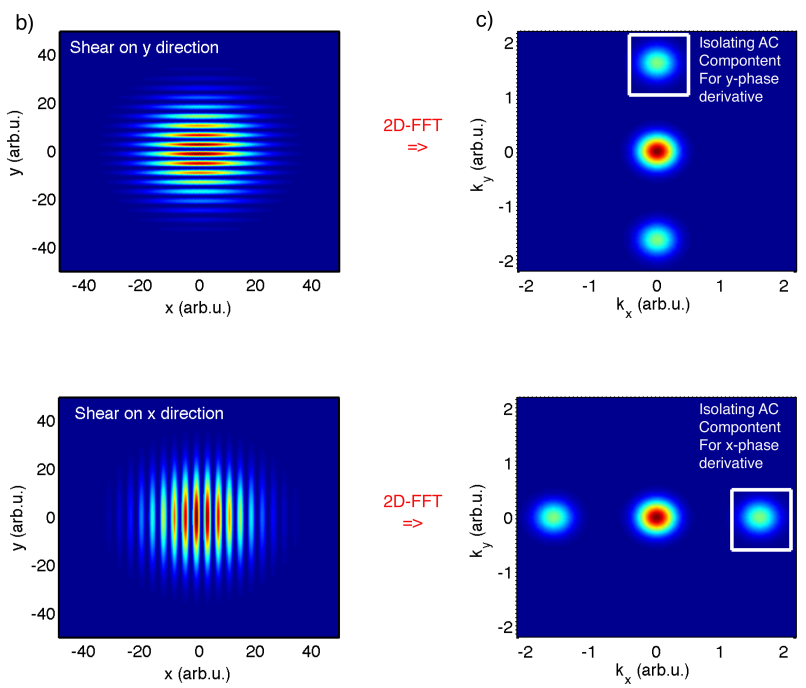

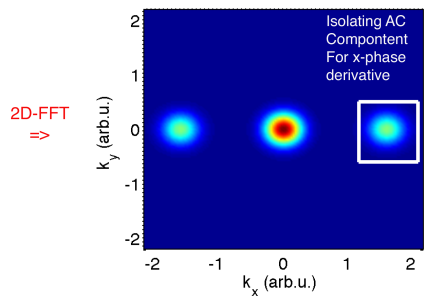

Figure 4.18: Lateral shearing interferometry technique in the Optics. Panel a) shows the diagrams for the lateral shearing on $y$ (upper plot) and $x$ (lower plot) directions. As it is denoted by the black and the orange circles, two copies of the laser beam are created that are lateral sheared one with respect to the other. The lateral shearing are indicated by $\Delta y$ and $\Delta x$. The upper and lower panels b) show the interferogram structures for both lateral shearing beams. In addition, the panel c) depicts the two dimensional Fourier transform (2D-FFT) of the interferograms shown in the panel b). In c) the white rectangles denote the positive AC component for the $y$ and $x$ shearing which encode the partial phase derivatives, $f_{y}(x, y)$ and $f_{x}(x, y)$. 
be approached to the partial derivatives of the phase, $\Delta \Phi_{y}(x, y) \approx \Delta y \frac{\partial \Phi(x, y)}{\partial y}$ and $\Delta \Phi_{x}(x, y) \approx \Delta x \frac{\partial \Phi(x, y)}{\partial x}$, on $x$ - and $y$-directions, respectively [130, 152]. For simplicity, we denote the derivative of the phase along $x$ and $y$ as $f_{x}(x, y)$ and $f_{y}(x, y)$, respectively.

From a mathematical viewpoint at least these two phase partial derivatives of the beam $\mathrm{A}_{\mathrm{F}}(x, y)$ are required to reconstruct the phase $\Phi(x, y)$ and therefore the wavefront is obtained. Then, this is the main reason to work with two different sheared interferograms.

To extract the partial derivatives from one of the interferograms three steps are followed: First, the 2D-FFT is employed on one of the interferograms, i.e., see upper plot in Fig. 4.18 c). Then, the positive-momentum $\mathrm{AC}$ component, $\mathrm{AC}_{k_{y}^{(+)}}\left(k_{x}, k_{y}\right)$, is isolated. In the case of the lateral shearing interferogram on $y$-direction, the momentum component is about $k_{y, 0}^{(+)}$. After filtering this $\mathrm{AC}$ component, it is moved to the zero momentum along $k_{y}$ [129]. We denote this shifted $\mathrm{AC}$ component as $\mathrm{AC}_{k_{y}^{(+)}}\left(k_{x}, k_{y}\right)$. Second, the two dimensional inverse Fourier transform (2D-IFFT) is done to the shifted

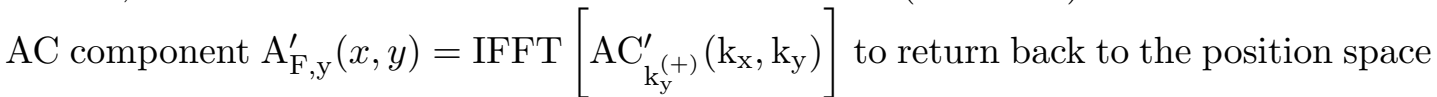
$(x, y)$. The third step is to compute the phase of $\mathrm{A}_{\mathrm{F}, \mathrm{y}}^{\prime}(x, y)$ that relates the partial derivative $f_{y}(x, y)$ of the phase along the $y$-direction. In order to recover the partial derivative $f_{x}(x, y)$, these three steps are also applied to the interferogram for the lateral shearing on the $x$-direction. The only difference is the isolated direction of the AC component that is now $k_{x, 0}^{(+)}$.

Once the two partial derivatives are extracted, the phase $\Phi(x, y)$ for the wavefront can be retrieved by integrating the gradient which contains $f_{x}(x, y)$ and $f_{y}(x, y)$.

To characterize the EWP, we will use the same APT of two pulses linearly polarized, but the streaked field will be circularly polarized. The main feature of this circularly polarized IR laser is that the two EWP copies can be lateral momentum sheared along both momentum-directions $p_{x}$ and $p_{y}$. In the case of the EWPs this effect will depend on the ionization time and the time delay between the APT and the circular IR laser field.

For a better understanding of the lateral shearing or lateral streaked EWP, the Fig. 4.19 depicts the basic idea for the lateral momentum-shearing on $y$-direction. The figure shows an APT in presence of a circular IR laser field and the two emitted EWPs with their final momentum streaked interferogram. The XUV attosecond pulses are linearly polarized along the $x$-direction.

When the first XUV laser pulse, $E_{\mathrm{X}}^{(1)}$, ionize the atom or molecule, the IR vector potential, $A_{\mathrm{L}, \mathrm{y}}(t)$, on the $y$ is maximum while the $x$ component at the same time, 
$A_{\mathrm{L}, \mathrm{x}}(t)$, is zero. Then, the momentum distribution $\mathrm{S}_{\mathbf{p}, 1}$ of EWP is streaked only along

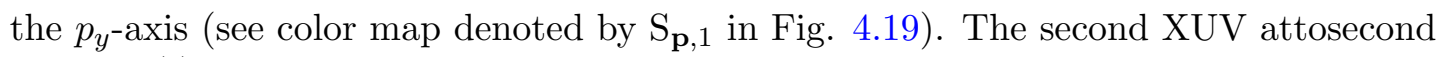
pulse, $E_{\mathrm{X}}^{(2)}$, is located at a time delay $\tau_{\mathrm{X}}$ of one IR cycle with respect to the first $\mathrm{XUV}$. This means that the second momentum distribution of the $\mathrm{S}_{\mathbf{p}, 2}$ EWP is created around a zero of the IR vector potential, $A_{\mathrm{L}, x}$, component and a small value of the $A_{\mathrm{L}, y}$. This is so, because the IR laser pulse has a ultra-short envelope. This scheme ensures that only the EWP will be sheared along the $y$ component in order to get the most cleaning partial momentum phase derivative along the $p_{y}$-axis. Otherwise the final 2D interferogram will contain a resulting shearing on $p_{x}$ and $p_{y}$-directions, which complicate the extraction of the phase of the partial derivative along the $p_{y}$.

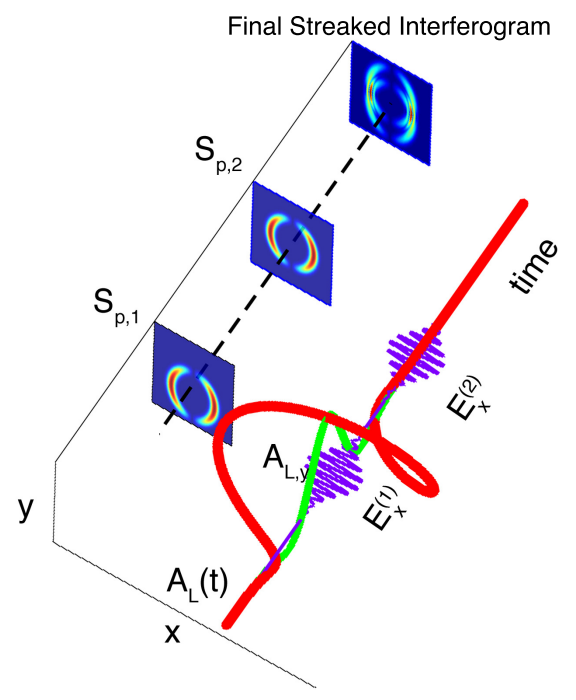

Figure 4.19: Lateral momentum-shearing for the EWPs interferometry scheme. Graphic representation of the two delayed EWPs denoted by $\mathrm{S}_{p, 1}$ and $S_{p, 2}$ (color scale maps) and the final streaked momentum interferogram. The photoionization is driven by the two delayed attosecond pulses $E_{\mathrm{X}}^{(1)}$ and $E_{\mathrm{X}}^{(2)}$ (violet solid line) in presence of a weak IR laser field (red solid line) which is circularly polarized. The XUV pulses are polarized along the $x$-axis. The circular vector potential of the IR is depicted in red solid line. The $y$-axis component of the vector potential of the IR laser field $\mathbf{A}_{\mathrm{L}}(t)$ is shown in green line. In this case, the EWP is time delayed by $\tau_{\mathrm{x}}=T_{0}$.

The lateral momentum-shearing on the $p_{x}$-direction can be easily constructed by changing the CEP of the IR laser pulse or the time delay between the APT an the IR.

So far, we have shown that the lateral momentum-shearing interferometry can be implemented for the EWP characterization phase in a 2D plane, analogous to the optical counterpart. However, we note that our implementation will be slightly different than the optical one. The main difference is that the momentum distribution of the EWP 
is located around a ring and not in a total disk like in the case of the optical wavefront characterization.

The next Section will be focused on defining the two lateral momentum-shearing interferograms needed to extract the full EWP partial phase derivatives by employing the SFA.

\subsubsection{Two dimensional electron wave packet interferometry}

While in the optical lateral shearing interferometry the shearing is introduced by a shearing plate [14], in our quantum version the control of the IR laser field polarization induces the desirable lateral momentum-shearing along the two perpendicular directions.

In order to demonstrate that two lateral shearings on $p_{x}$ and $p_{y}$-directions can be induced in the EWPs, we integrate numerically the differential form of the SFA, Eq. (2.95), for the ionization complex transition amplitude $a\left(\mathbf{p}, \tau_{1}, \tau_{2}\right)$. The attosecond streaking effect and QSPIDER conditions are taken into account for those calculations. In addition, we have assumed that the APT of two pulses are polarized along the $x$-axis and the IR laser field is circularly polarized in the $x y$ plane.

We chose the $2 p$ state of $\mathrm{He}^{+}$as an initial example. The orbital is oriented along the $y$-direction and therefore the corresponding bound-free dipole transition matrix element $d_{2 p}(\mathbf{p})=-\left\langle\mathbf{p}|x| \Psi_{2 p}\right\rangle$ reads:

$$
d_{2 p}(\mathbf{p})=1536 \frac{Z^{7 / 2}}{\pi} \frac{p_{x} p_{y}}{\left(Z^{2}+4 \mathbf{p}^{2}\right)^{4}},
$$

where $Z=2 \sqrt{2 I_{p}}$ in the case of the $2 p$ state for the $\mathrm{He}^{+}$[140]. The ionization potential of the $2 p$ orbital is about $I_{p}=0.5$ a.u. Note that this bound-free dipole transition matrix element has two perpendicular nodes, namely one along the $x$-axis and other on the $y$-axis. It is important to mention that the node on the $x$-direction brings structural information about the orientation of the $2 p$ orbital. The node on the $y$-direction brings information about the coupling between the XUV polarization and the orbital $2 p$.

The APT parameters are as follows: peak intensity $I_{\mathrm{X}}=5 \times 10^{12} \mathrm{~W} / \mathrm{cm}^{2}$, central frequency $\omega_{\mathrm{X}}=2.0$ a.u., and $\mathrm{FWHM}=175$ and $\mathrm{CEP}=\pi / 2$ for the first XUV pulse $E_{\mathrm{X}}^{(1)}(t)$, and the same peak intensity and time duration for the second $E_{\mathrm{X}}^{(2)}(t)$, but now the $\mathrm{CEP}=-\pi / 2$. Both attosecond pulses are polarized along the $x$-axis. As an example, the time delay $\tau_{\mathrm{x}}$ between the two attosecond pulses is fixed to $\tau_{\mathrm{x}}=T_{0} / 2$. The 
IR laser field is circularly polarized and its parameters are: peak intensity $I_{0} \sim 10^{13}$ $\mathrm{W} / \mathrm{cm}^{2}$, central frequency $\omega_{0}=0.057$ a.u., FWHM $\sim 7$ fs with $\sin ^{2}$ envelope and $\mathrm{CEP}=0$.

It is important to mention that the time delay $\tau_{\mathrm{X}}$ is chosen to half IR cycle because the first EWP will be streaked on the negative (or positive) $p_{y}$-direction and the second EWP, on the positive (or negative) $p_{y}$-direction, respectively. This momentum streak will depend on the relative time delays, $\tau_{1}$ and $\tau_{2}$, between the APT and the IR laser field. Thus, under this scheme, the lateral shearing on $p_{y}$-direction is constructed in order to get the first interferogram which contains the EWP phase partial derivative along $y$. Fig. 4.20 shows the results using SFA under both lateral momentum-shearing in perpendicular directions.

First of all and for comparison, we have computed the final momentum distribution for the photoionization driven by a single XUV attosecond pulse (see upper plot in the Fig. 4.20a)). It is important to note that the momentum bandwidth sets the region (in this case a ring), where we recover the dipole phase. Width rings of the lateral momentum-shearing interferograms, which are induced by the circular IR laser field on both directions, are also compared.

The upper plot in Fig. 4.20b) shows the pulses for the lateral shearing simulation on the $y$-direction. The lower plot shows the coherent superposition of the two EWPs or lateral momentum-shearing interferogram along the $y$-direction. As it is shown by the green circle lines, a large streak on each EWP is induced by the $y$-component of the circularly polarized IR laser field. For instance, the first EWP is upper momentumshifted by an amount $\Delta p_{y}=-A_{\mathrm{L}, y}\left(\tau_{1}\right)=A_{0}$.

The second one, on the other hand, is down momentum-shifted by $\Delta p_{y}=-A_{\mathrm{L}, y}\left(\tau_{2}\right)=$ $-A_{0}$. Here, $A_{0}$ denotes the maximum amplitude of the IR vector potential $\mathbf{A}_{\mathrm{L}}(t)$. Then, the total lateral momentum-shearing on $y$-direction will be defined as the difference $\Delta A_{\mathrm{L}, y}=A_{\mathrm{L}, y}\left(\tau_{2}\right)-A_{\mathrm{L}, y}\left(\tau_{1}\right)$.

Note that for this lateral momentum-shearing, $\Delta A_{\mathrm{L}, y}$, the final interferogram fringes are limited to the overlap regions between both streaked EWPs. Thus, it is important to control the lateral momentum-shearing with the purpose to obtain the maximum momentum overlap region. This will allow us to recover the partial derivative on a larger region where the EWPs overlap.

The lateral momentum interferogram on the $x$-axis is depicted in Fig. 4.20c). This coherent EWPs superposition is obtained by changing the relative time delays, $\tau_{1}$ and $\tau_{2}$, between the APT and IR laser field to a total amount of $T_{0} / 4$ (see upper plot in Fig. $4.20 \mathrm{c})$ ). In this case, the first EWP is only momentum-shifted to the negative $x$ - 

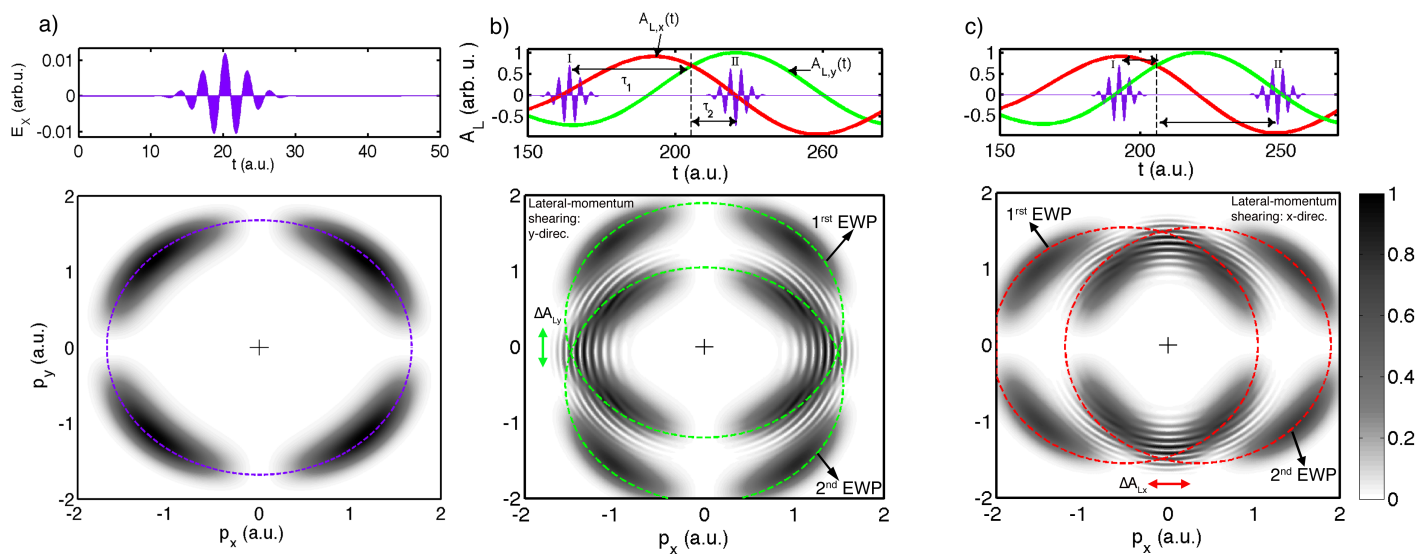

Figure 4.20: 2D EWPs interferometry. a) The single attosecond pulse $E_{\mathrm{X}, x}(t)$ in the upper plot is depicted in the violet area. In the lower plot the corresponding final momentum distribution $\mathrm{S}_{e}\left(p_{x}, p_{y}\right)=|a(\mathbf{p})|^{2}$ of the emitted EWP from the $2 p$ orbital of $\mathrm{He}^{+}$. The violet dashed circle line indicates the radial central photoelectron momentum $p_{0}=\sqrt{2\left(\omega_{\mathrm{X}}-I_{p}\right)}$. The circular IR vector potential components on the $x$-direction $\left(A_{\mathrm{L}, x}(t)\right.$ red line) and on the $y$ direction $\left(A_{\mathrm{L}, y}(t)\right.$ green line) are shown in the upper graphs of b) and c), respectively. The APT fields are also depicted in violet areas. In both graphs b) and c), the time delay $\tau_{\mathrm{x}}=\tau_{2}-\tau_{1}=\frac{T_{0}}{2}$ is fixed. The relative time delays $\tau_{1}$ and $\tau_{2}$ between the APT and the IR are changing as it is pointed out in b) and c). The final lateral momentum-shearing interferograms on $p_{y}$ and $p_{x}$-directions are shown in the lower plots of b) and c), respectively. The corresponding green dashed circle lines in $\mathrm{b})$ are centered at the momentum vector $\left(0, \pm \Delta p_{y}\right)$, this is at the points $\left(0, \pm A_{0}\right)$. A similar behavior is shown in the red dashed circles of $\left.\mathrm{c}\right)$, but here the circles are centered at $\left(\mp \Delta p_{x}, 0\right)$. In $\left.\mathrm{b}\right)$ and $\left.\mathrm{c}\right)$, the radius of these circles is the same and it is $p_{0}$. In all the lower plots a-c) the black crosses denote the momentum origin $(0,0)$. These momentum distributions were calculated by the numerical integration of Eq. (2.95). 
direction, $\Delta p_{x}=-A_{\mathrm{L}, x}\left(\tau_{1}\right)=-A_{0}$, and the second EWP one, to the positive, $\Delta p_{x}=$ $-A_{\mathrm{L}, x}\left(\tau_{2}\right)=A_{0}$. This is clearly shown with red dashed circles in Fig. 4.20c). Thus, we define the lateral momentum-shearing on the $x$-direction as $\Delta A_{\mathrm{L}, x}=A_{\mathrm{L}, x}\left(\tau_{2}\right)-A_{\mathrm{L}, x}\left(\tau_{1}\right)$.

Our numerical SFA results suggest that a scheme of two lateral momentum-shearing interferograms, one on the $x$ - and another one on the $y$-direction, is essential to extract the EWP phase gradient and therefore, the dipole phase gradient as well. These two coherent EWPs interferograms are based on the ionization driven by the APT linearly polarized in presence of a weak circularly polarized IR laser field.

The next Section will be dedicated to explain the retrieval algorithm and the best conditions for the implementation of the lateral momentum-shearing interferometry QSPIDER technique.

\subsubsection{QSPIDER and 2D lateral momentum-shearing implementation}

One of the steps which deserves special attention in the lateral momentum-shearing technique is the overlap momentum region. As described before, the lateral momentumshearing can be manipulated by controlling the IR laser field, i.e., by changing the IR peak intensity.

In this Section we review the steps to extract the EWP and dipole phase gradients. In addition, we define the most convenient IR intensity range in order to build the interferograms with the maximum possible overlap between the emitted two EWPs. It is also important to choose the most convenient time delay $\tau_{\mathrm{x}}$ between the two attosecond pulses in order to well separate the DC and AC components.

As an example, figure 4.21 depicts the SFA calculations for the QSPIDER with lateral momentum-shearing on the $y$-direction and the first steps for the EWP amplitude and EWP phase partial derivative reconstructions. The chosen complex dipole transition matrix element is the same as in the Section 4.3.1 defined in Eq. (4.29). The top plot shows the $x$ - and $y$-directional components of the IR vector potential $\mathbf{A}_{\mathrm{L}}(t) \equiv\left(A_{\mathrm{L}, x}(t), A_{\mathrm{L}, y}(t)\right)$ and the electric field $E_{\mathrm{X}}(t)$ of the APT which are linearly polarized along $x$-axis.

The APT parameters (for both XUV pulses) are: central frequency $\omega_{\mathrm{X}}=2.0$ a.u., peak intensity $I_{\mathrm{X}}=5.0 \times 10^{12} \mathrm{~W} / \mathrm{cm}^{2}, \mathrm{FWHM} \sim 175$ and $\mathrm{CEP}= \pm \frac{\pi}{2}$. For the first (second) XUV, the sign is positive (negative). As it is denoted on the top of Fig. 4.21, the time delay between the XUVs is fixed to $\tau_{\mathrm{x}} \sim T_{0}$. We have found that this is the most convenient time delay to separate adequately the AC from DC components, and most importantly for extracting the EWP partial phase derivative on the $y$-direction 


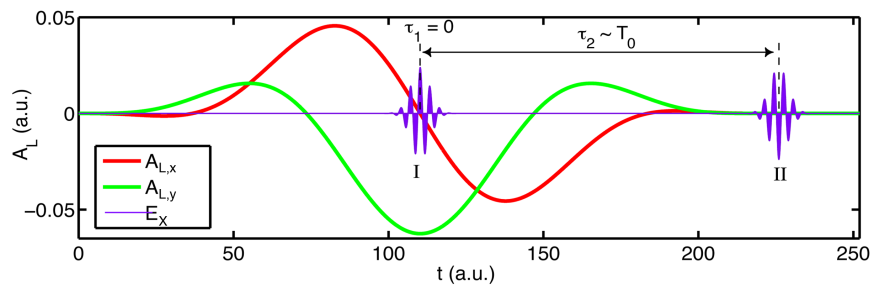

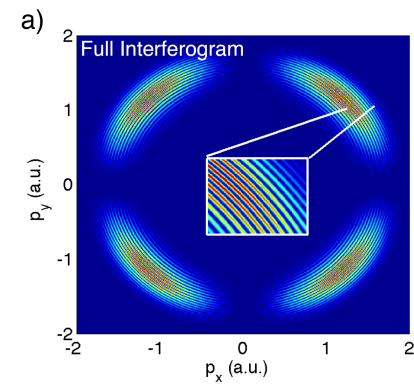

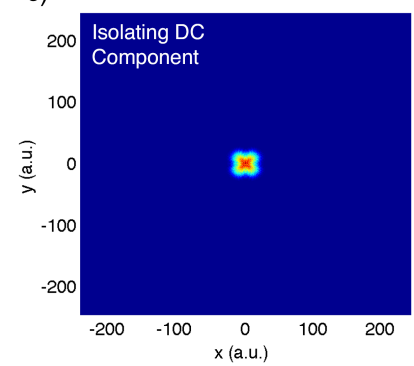

b)

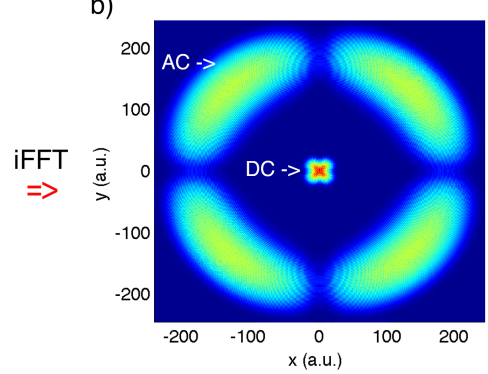

d)

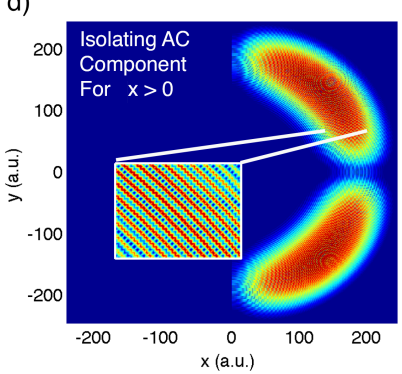

Figure 4.21: 2D EWPs lateral momentum-shearing along $y$-direction. On the top, the two IR vector potential components $A_{\mathrm{L}, x}(t)$ (red line) and $A_{\mathrm{L}, y}(t)$ (green line), and the APT $E_{\mathrm{X}, x}(t)$ (in violet areas) which are linearly polarized along $x$-direction. In a), the final full lateral momentum-shearing interferogram $\mathrm{S}_{e}\left(p_{x}, p_{y}, \tau_{\mathrm{x}}\right)$ along $y$-direction for the coherent superposition of the two emitted EWPs in presence of the circularly IR laser field. The insets show a small region of the interferogram to highlight the fringes. In the plot b) we depict the absolute value of the $\operatorname{IFFT}\left[\mathrm{S}_{e}\left(p_{x}, p_{y}, \tau_{\mathrm{x}}\right)\right]$ as a function of the pseudo-position domain $(x, y)$. We also indicate the DC and AC components. c-d) The isolated DC component and positive AC component for $x>0$. The inset in d) shows a zoomed space region. 
(see Fig. 4.21b)). The circularly polarized IR laser parameters are: central frequency $\omega_{0}=0.057$ a.u., peak intensity $I_{0}=5.0 \times 10^{11} \mathrm{~W} / \mathrm{cm}^{2}, \mathrm{FWHM} \sim 4$ fs and CEP $=0$. The envelope of the IR laser pulse is sine-squared.

The maximum overlap between the two streaked EWPs is obtained at the peak IR intensity $I_{0}$. Then, the induced lateral momentum-shearing is $\Delta A_{\mathrm{L}, y}(t)=0.062$ a.u. This relative shearing is small enough to approach the EWP phase different to the partial derivative of the phase.

The momentum grid parameters on the $x$-axis are: momentum length $L_{p_{x}}=10$ a.u., and momentum step $\Delta p_{\mathrm{x}}=0.004$ a.u. The same momentum grid parameters are chosen for the $y$-direction.

As it is also mentioned in the QSPIDER technique, the momentum shearing should be comparable to the momentum grid step. However, $\Delta A_{\mathrm{L}}$ in general cannot be smaller than $\Delta p_{\mathrm{x}}$. Otherwise, the numerical accuracy is not able to ensure a good partial derivative of the EWP over the defined momentum grid range.

Fig. 4.21a) shows the final interferogram of the coherent superposition of the two emitted EWPs by the above described pulses. The ring of interference fringes shows a very good overlap of the two EWP copies. The inset plot depicts a small momentum range (white lines) needed to observe the interference fringes.

Once the interferogram is calculated, we are in the position to apply the QSPIDER technique or the lateral momentum-shearing interferometry algorithm. Thus, Fig. 4.21b) plots the 2D inverse Fourier transform of the full EWPs momentum interferogram as a function of the pseudo-position space $(x, y)$. Both components DC and $\mathrm{AC}$ are indicated in the picture. As it is noted both the DC and $\mathrm{AC}$ components are very well separated from each other.

Figs. 4.21c-d) depict the isolated DC components and AC positive component for $x>0$ along all the $y$-range, respectively. In other words, we have chosen the first and fourth quadrants of the pseudo-position coordinate system for the positive AC component.

We isolate the $\mathrm{AC}^{+}$component in order to retrieve the EWP phase derivative of the structural node on the $x$-direction. This node comes from the $2 p$ orbital. In this way, we find that the lateral momentum-shearing algorithm will be different to the optical technique. The momentum interference fringes of the EWPs exhibit a radial interferogram behaviour and not a parallel to $x$ - or $y$-axes like in the Optics.

Furthermore, we have isolated the first and second quadrants as a "positive" AC component (for $y>0$ and for all $x$ ) in order to retrieve the phase gradient along the $y$-axis. Thus, we should expect to extract the dipole phase derivative along $y$-axis. This was not 
the case. However, the best one positive AC momentum range is shown in Fig. 4.21d). As we will see, this region exhibits a nice derivative retrieval of the node along $x$-axis, as it would be easily expected.

To extract the EWP amplitude and the EWP phase derivative along $y$-axis, we will go back to the momentum domain. Then, a FT is done on the complex DC component $A(\mathbf{p})=$ FFT $[\mathrm{DC}(\mathrm{x}, \mathrm{y})]$ (see Fig. 4.21c)) and on the complex AC component $A_{\phi}(\mathbf{p})=\mathrm{FFT}\left[\mathrm{AC}^{+}(\mathrm{x}, \mathrm{y})\right]$. Fig. 4.21d) shows these quantities. Then, to extract the full EWP amplitude as if a single EWP was created, we remove the shift induced by the IR and divide the resulting amplitude by the spectrum of the a SAP.

The EWP phase difference $\Delta \phi_{d, y}(\mathbf{p}) \approx \Delta A_{\mathrm{L}, y} \frac{\partial \phi_{d}(\mathbf{p})}{\partial p_{y}}$ is recovered also by removing the time-dependent phase differences from the phase of $A_{\phi}(\mathbf{p})$. The phases to be removed are:

(i) The Volkov phase difference, Eq. (4.25),

(ii) The so-called atomic phase difference $\Delta \phi_{a}=\left(\tau_{2}-\tau_{1}\right) I_{p}=\tau_{\mathrm{x}} I_{p}$,

(iii) The LIC phase difference $\Delta \phi\left(\mathbf{p}, \tau_{1}, \tau_{2}\right)$, which can be obtained from Eq. (4.16),

(iv) The XUV attosecond phase difference $\Delta \phi_{\mathrm{X}}\left(\omega_{\mathbf{p}}\right)$.

Finally, the same described steps are applied to the lateral momentum-shearing interferogram on the $x$-orientation. This interferogram is computed by changing the time delays $\tau_{1}$ and $\tau_{2}$ of the XUV pulses with respect to the IR laser field but fixing $\tau_{\mathrm{x}}=\tau_{2}-\tau_{1} \sim T_{0}$. This means that the time delays $\tau_{1}$ and $\tau_{2}$ are changed about $T_{0} / 4$, i.e.:

$$
\begin{gathered}
\tau_{1} \rightarrow \tau_{1} \pm \frac{T_{0}}{4} \\
\tau_{2} \rightarrow \tau_{2} \pm \frac{T_{0}}{4} .
\end{gathered}
$$

This guarantee that the lateral momentum-shearing will be along the $x$-axis. Another scheme that can be applied to induce the shearing along the $x$-direction, would be to change the CEP of the IR laser field and to fix the time delays between the APT and the IR laser field. Finally, with this lateral momentum-shearing interferogram on the $x$-axis, we are able to extract the EWP phase gradient on the $x$-direction and by following similar steps to retrieve the gradient on $y$-direction. The main difference will be in the isolated $\mathrm{AC}$ component: the region will be now defined by the first and second quadrants in case of the extracted phase difference analysis for the lateral shearing along $x$-axis.

Once we have the two partial derivatives of the phase $\left(\frac{\partial \phi_{d}(\mathbf{p})}{\partial p_{x}}, \frac{\partial \phi_{d}(\mathbf{p})}{\partial p_{y}}\right)$ the EWP 
phase or dipole phase can be recovered by integrating this gradient.

\subsubsection{Results within SFA and TDSE models}

In the next Sections we will describe how to apply the lateral momentum-shearing QSPIDER technique by using the SFA and the TDSE. The SFA works very good for potentials where the influence of the Coulomb tail in the photo-ionization process, in presence of a weak IR laser field, is negligible. We will calculate the momentum distribution of the EWP, by using SFA for the two lateral momentum interferograms as is described above.

As an example we chose two bound states. These are the $2 s$ and the $2 p$ orbitals of the $\mathrm{He}^{+}$ion atom. The dipoles of the problem will be the bound-free transition from the $2 s$ and $2 p$ states to the continuum described by Eqs. (4.26) and (4.29), respectively.

We will compute the two final lateral momentum-shearing interferograms by employing our 2D TDSE to the $2 p$ orbital in an atom described by a short-range potential.

\subsubsection{Lateral momentum-shearing results within SFA}

In order to test the QSPIDER lateral momentum-shearing interferometry technique, two examples are chosen. These are: the bound-free transition given by the equations (4.26) and (4.29). These dipoles are transitions from the $2 s$ and $2 p$ states to the continuum. We denote them by $d_{2 s}(\mathbf{p})$ and $d_{2 p}(\mathbf{p})$, respectively.

These calculations are performed under the assumption that the APT are linearly polarized on the $x$-axis. The complex dipoles are depicted in Fig. 4.22.

In particular, the amplitude and phase for $d_{2 s}$ are depicted in a) and c), respectively. The same in b) and d) but here for $d_{2 p}$. In the case of $2 s$, two different nodes emerge: the first one is a vertical line at $p_{x}=0$ along $p_{y}$-axis; the second one is a circular node which denotes a circle of radius $p_{r n}=1.41 \mathrm{a}$.u. The phase of the dipole $d_{2 s}$ also reflects the structure of the different nodes in the dipole amplitude. The phase structure exhibits different phase jumps of $\pi$ which can be seen in Fig. 4.22c). It is important to point out that the radial node encodes information of the orbital shape for $2 s$ state, meanwhile the vertical node along $p_{y}$-axis carries out information about the coupling between the XUV and the $2 s$ state. This is so because the XUV is polarized on the $p_{y}$-axis. Then, information about the attosecond XUV polarization is also given by this node along the $p_{y}$-orientation.

Figs. 4.22b) and d) show the dipole amplitude and the dipole phase for the transition $2 p$-continuum, respectively. The amplitude depicts two perpendicular nodes: one 

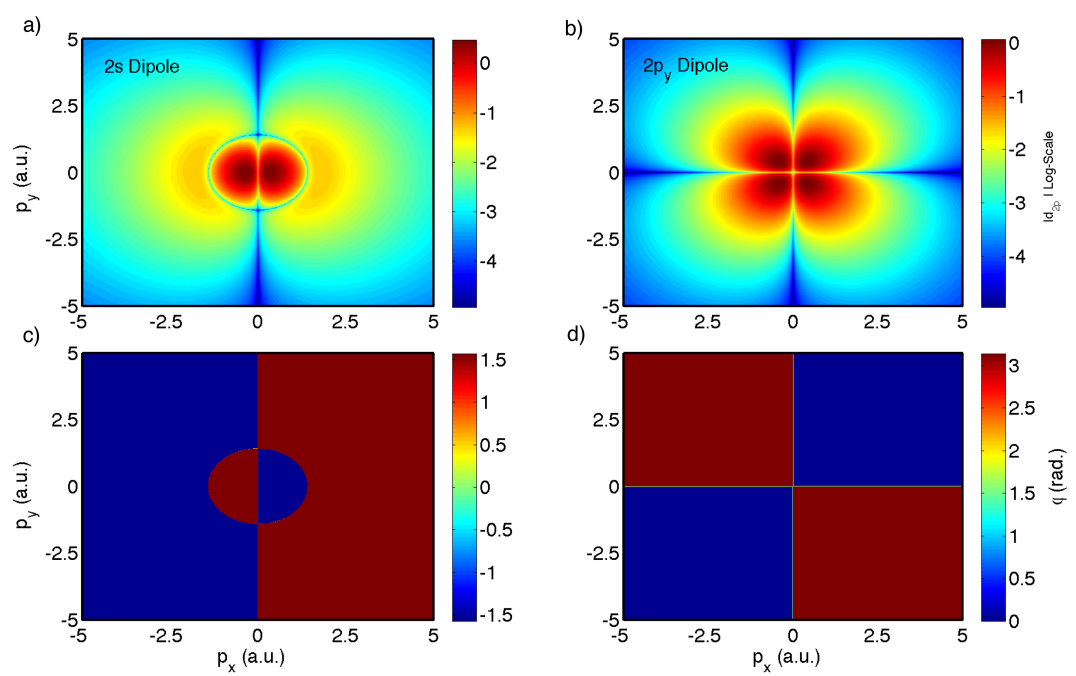

Figure 4.22: Complex dipole for the $2 s$ and $2 p$ states. a) And b) depict a 2D cut on the momentum plane $\left(p_{x}, p_{y}\right)$ of the dipole transition matrix element amplitude for the transition from the $2 s$ and $2 p$ states to the continuum $|\mathbf{p}\rangle$, respectively. c-d) show the corresponding dipole phases.

is along $p_{x}$-axis and the other one on the $p_{y}$-axis. The horizontal node denotes information about the $2 p$ state. Thus, we can infer that the orbital is oriented along $y$-direction. The dipole phase structure also exhibits phase jumps of $\pi$ between each one of the quadrants. It is interesting to point out that these phase jumps also encode information about the structure of the orbital $2 p$ as well as the interaction between the XUV and the orbital.

\section{QSPIDER momentum-spatial shearing in the $2 p$}

Here we will show the first results of the emitted EWP characterization from the $2 p$ orbital of the $\mathrm{He}^{+}$atom as well as its associated complex dipole. The EWP amplitude and phase retrieval is done by following the steps described in the Section 4.3.2.

In order to compute the lateral momentum-shearing interferograms on the $p_{y^{-}}$and $p_{x}$-directions we numerically integrate the SFA equation (2.95) for the QSPIDER lateral shearing conditions. The two attosecond pulses parameters as well as the circularly polarized IR laser to build the interferogram calculations are the same as those mentioned in Section 4.3.2.

In fact, we use the same calculated interferogram for the lateral momentum-shearing on the $y$-axis described in the Section 4.3.2. The interferogram on the $x$-direction is also computed under the same conditions, but here we have changed the time delay 
parameters $\tau_{1}$ and $\tau_{2}$ according to Eq. (4.30).

Then, we apply the QSPIDER algorithm to extract both the EWP amplitude and EWP phase. We reconstruct the dipole amplitude, by dividing the recovered EWP amplitude by the spectrum of a SAP. The dipole phase is just obtained by integrating the retrieved phase gradient. The QSPIDER lateral momentum-shearing reconstruction EWP and dipole are depicted in Fig. 4.23.
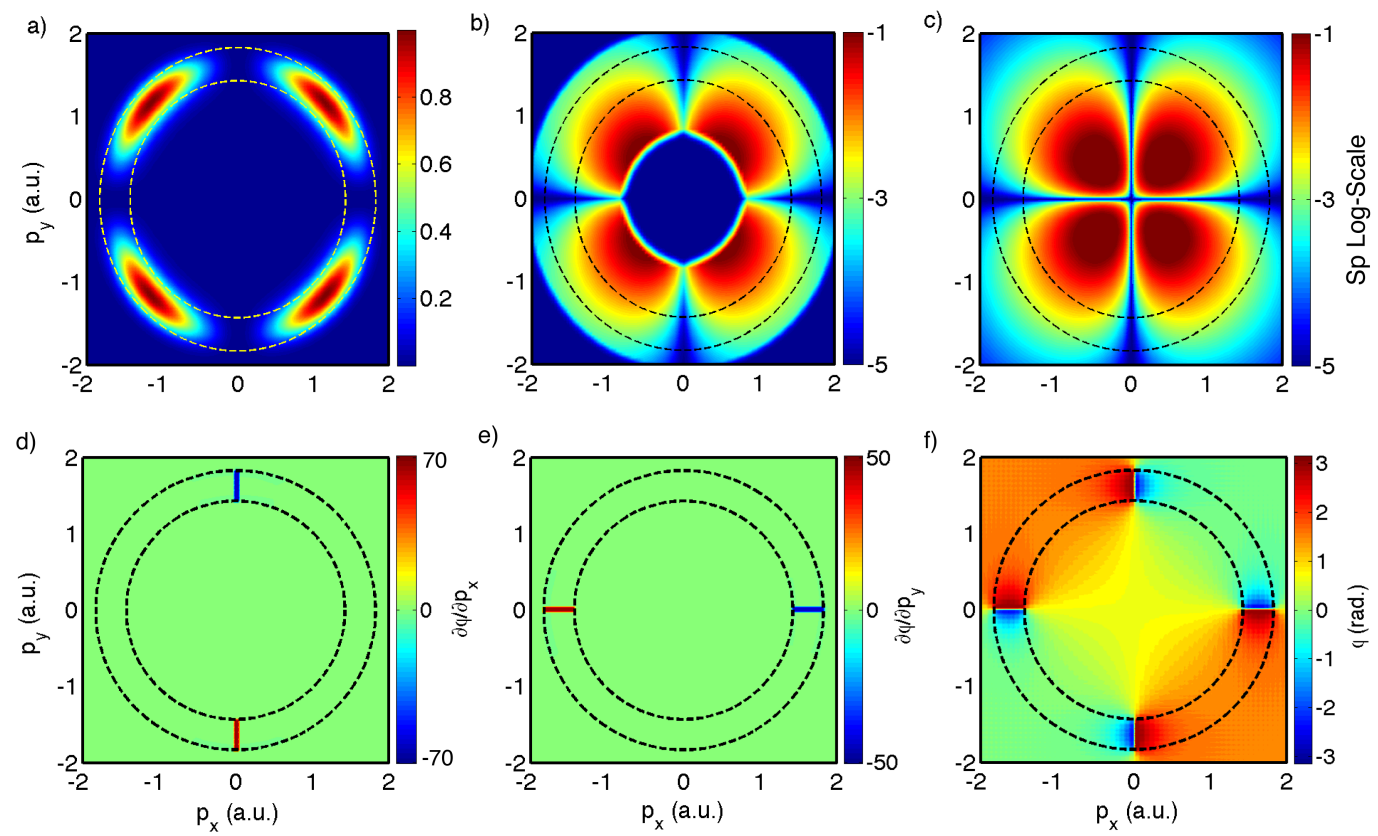

Figure 4.23: QSPIDER for the lateral momentum-shearing: EWP and 2pdipole. Plot a) shows the retrieval momentum distribution EWP amplitude $|a(\mathbf{p})|^{2}$ (color scale) from the lateral momentum-shearing interferogram technique. The reconstructed dipole amplitude $\left|d_{2 p, \mathrm{R}}(\mathbf{p})\right|$ in b) compared to the exact dipole amplitude $\left|d_{2 p}(\mathbf{p})\right|$ c), both in logarithm scale. The reconstructed QSPIDER EWP phase or dipole phase partial derivatives along $p_{x}$ and $p_{y}$ are shown in d) and e). The integrated gradient of the phase (d-e) is depicted in f). The white and black dashed circles denote the EWP momentum bandwidth retrieval range.

The momentum grid parameters to perform such calculations are the same as the ones used in Section 4.3.2 and the lateral momentum-shearing on each directions are: $\left(\Delta A_{\mathrm{L}, x}=0.045, \Delta A_{\mathrm{L}, y}=0.062\right)$.

First of all, the EWP amplitude around the retrieval region-range defined by the two white circles, is shown in a). This amplitude exhibits two perpendicular nodes which, according to the dipole $d_{2 p}(\mathbf{p})$, are expected. Also Fig. 4.23b) draws the recon- 
structed dipole amplitude. This was obtained by dividing the EWP amplitude by the "momentum" spectra of a single XUV attosecond pulse amplitude. For comparison the exact dipole amplitude is depicted in c). We find a very good agreement between the retrieved dipole amplitude and the exact one around the momentum ring defined by the spectrum of a single XUV attosecond pulse used in our example (see the black dashed circles of both Figs. 4.23b-c)).

The retrieved partial derivatives of the dipole phase along the $p_{x^{-}}$and $p_{y}$-directions are also depicted in Fig. 4.23d) and e). These are in very good agreement with the expected derivative of the dipole phase under the retrieval momentum-range denoted by the two black dashed circles. It is important to point out that in order to retrieve the partial derivative of the dipole phase for the region $p_{x}<0$, we restrict the $\mathrm{AC}^{(-)}$ component for the lateral momentum-shearing analysis to the $y$-direction. A similar procedure is followed in case of the lateral momentum-shearing on the $x$-direction.

The retrieved gradient of the phase has been interpolated in a new momentum grid with the momentum steps defined by the two perpendicular $\left(\Delta A_{\mathrm{L}, x}, \Delta A_{\mathrm{L}, y}\right)$ lateral shearing. Then we integrate this phase gradient and reconstruct $\phi_{d_{2 p}}(\mathbf{p})$ utilizing the algorithm described in [153]. The result is shown in Fig. 4.23e). The retrieved phase is in very good agreement with the exact one (see Fig. 4.22c)) within the EWP momentum bandwidth. We note that a small deviation can be observed around the two perpendicular nodes. This may come from small numerical fluctuations of the interpolation algorithm. This is so, because we have interpolated the retrieval gradient on two new axis with the step shearing $\left(\Delta A_{\mathrm{L}, x}, \Delta A_{\mathrm{L}, y}\right)$. The most important feature, besides of this small fluctuation, is that the retrieval has a jump phase on the expected directions.

\section{QSPIDER momentum-spatial shearing in $2 s$}

The second example we present is for the ionization coming from the $2 \mathrm{~s}$ orbital of the helium ion. The calculation is done in the same way as in the reconstruction of the $d_{2 p}(\mathbf{p})$ dipole. However, in order to test if this new implementation of QSPIDER works with circular nodes too, we choose the following APT parameters: XUV central frequency $\omega_{\mathrm{X}}=1.65$ a.u., FWHM 212 as and peak intensity $I_{\mathrm{X}}=5.0 \times 10^{12} \mathrm{~W} / \mathrm{cm}^{2}$. Both attosecond pulses are the same but only their CEP is different, namely $\mathrm{CEP}= \pm \frac{\pi}{2}$. The IR laser parameters are the same as those used in the calculations presented in Fig. 4.23. It is also important to point out that the lateral momentum-shearing is the same as the one shown in the retrieval example for the $2 p$ orbital. We compute the two lateral momentum-shearing interferograms using the structure for the pulses depicted in Fig. 4.21. By applying the lateral momentum-shearing algorithm, we reconstruct 
the complex EWP and the complex dipole.

Fig. 4.24 shows the complex EWP and dipole reconstruction of the emission from the $2 s$ orbital. Fig. $4.24 \mathrm{a}$ ) depicts a very good retrieval of the EWP amplitude as in the case that a single XUV attosecond pulse ionizes the system. Note that the two nodes in the Fig. 4.22a) are observed over the momentum bandwidth denoted by the white dashed circles.
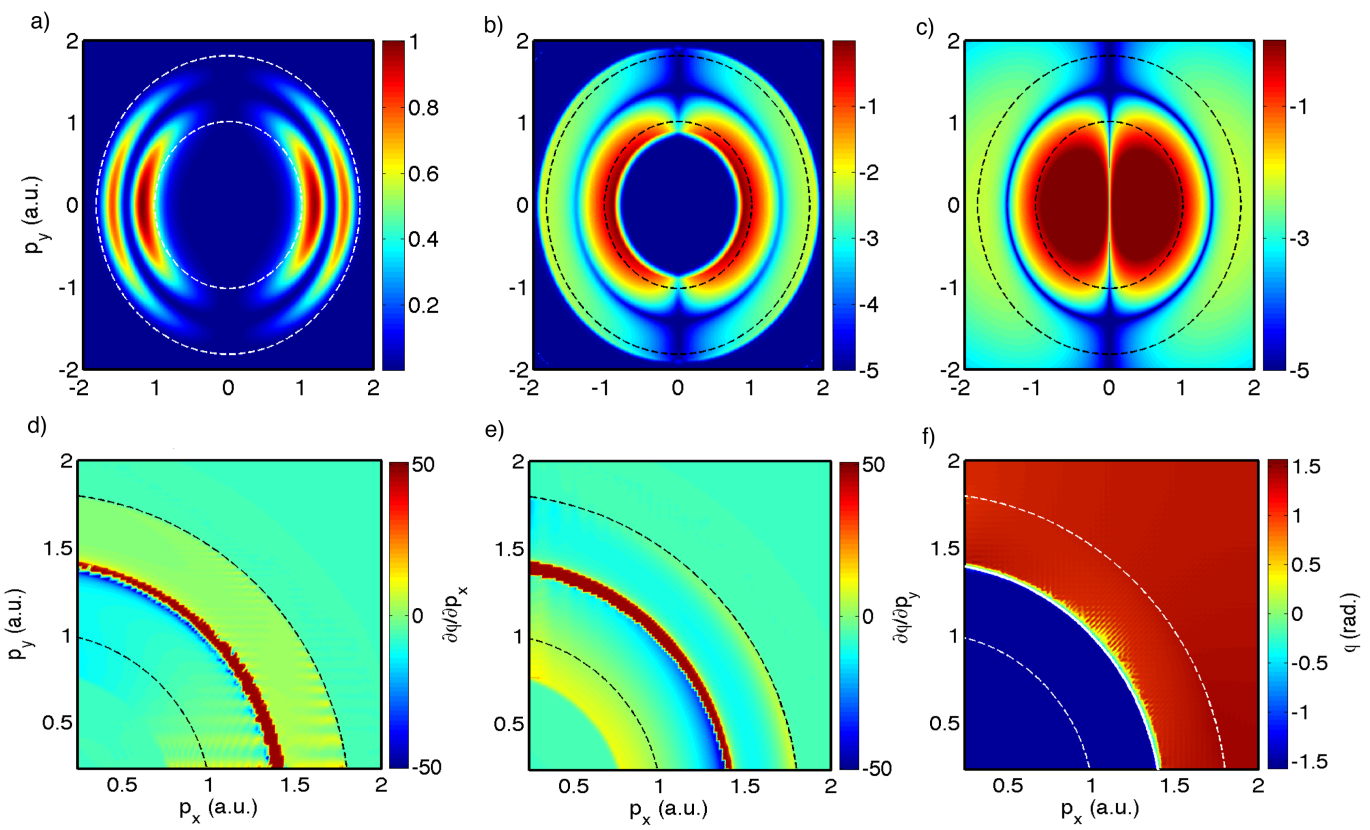

Figure 4.24: QSPIDER for the lateral momentum-shearing: EWP and 2sdipole. This figure shows the retrieval EWP amplitude, the QSPIDER extracted dipole amplitude, the exact dipole amplitude, the QSPIDER lateral momentum-shearing retrieval gradient of the dipole phase and the reconstructed dipole phase in plots a), b), c), d) and e), respectively. The retrieval phase gradient on the perpendicular directions and the integrated phase is only shown within the first quadrant. White and black dashed circles denote the EWP momentum range of retrieval. All of these plots are for the ionization coming form the $2 \mathrm{~s}$ orbital of the helium ion atom.

The Fig. 4.24b) shows the retrieval dipole amplitude $\left|d_{2 s, \mathrm{R}}(\mathbf{p})\right|$. We compare this amplitude with the exact one which is depicted in c). A very good agreement between the reconstructed dipole amplitude and the exact one is found. As expected the two nodes are very well retrieval. The momentum-radial node is about the circle with radius $p_{r n}=1.4$ a.u. The vertical node is located at $p_{x}=0$ along $p_{y}$-direction. These two facts are also in very good agreement with the exact dipole amplitude, too. 
In order to retrieve the dipole phase $\phi_{d_{2 s}}(\mathbf{p})$, first we reconstruct the phase gradient or the phase partial derivatives along both perpendicular momentum directions $\left(p_{x}, p_{y}\right)$. The results are depicted in Fig. 4.24d) and e), respectively. A structure close to a radial Dirac delta distribution is found for both partial derivatives. For simplicity only the first momentum quadrant has been drawn.

Finally, we numerically integrate these two partial derivatives [153] in order to recover the dipole phase. The result is depicted in Fig. 4.24e). We find a very good agreement with the exact one (see Fig. 4.22c)) over the momentum range defined by the spectrum of a single attosecond pulse.

According to our two examples to retrieve the complex EWP and complex dipole, we can conclude that the QSPIDER lateral momentum-shearing interferometry technique works very good. The ionization driven by the two attosecond pulses in presence of a circularly IR laser can be employed to extract structural information about the photoionization process.

So far, our retrieval scheme has been based on the SFA. In the next Section we will apply the same implementation of the retrieval EWP and dipole, but in this case utilizing a full 2D TDSE.

\subsubsection{Time dependent Schrödinger equation model}

In this Section, we will present our 2D-TDSE numerical calculations for the lateral momentum-shearing interferometry technique. First of all, we choose the $2 p$ state of a short-range Yukawa potential as a test system. Our choice is based on the fact that the PWs are a very good approximation for the continuum states of such a short-range potential. In particular, this potential can be written:

$$
V_{0}(r)=-Z \frac{\exp \left[-\frac{r}{a_{s}}\right]}{\sqrt{a+r^{2}}}
$$

where $a_{s}$ is the screening length, $a$ a soft-core parameter and $Z$ the charge of the potential. The vector $r=\sqrt{x^{2}+y^{2}}$ is defined in the plane $(x, y)$. We have chosen $Z=2$ in order to match the charge of the helium ion and the other parameters used in the simulation are: soft-core $a=0.8$ a.u. and screening charge $a_{s}=2$ a.u. We calculate the $2 p$ on the $y$-orientation by imaginary time propagation. The complex time-step is $\Delta t=-i 0.05$ a.u., and the spatial grid is a square box with the $L_{x}=L_{y}=300$ a.u. The grid step in each direction is $\Delta x=\Delta y=0.3$ a.u. For this potential the ionization potential is found to be $I_{p} \sim 0.1$ a.u. We stress that it is difficult to match the 
ionization potential of the $2 p$ orbital, computed using a Yukawa-like potential, to the actual experimental data. Here we have chosen this orbital with the aim to describe the general characterization of an EWP utilizing an exact TDSE calculation. Under this point of view, we are interested in the mechanisms which are comprised in a short-range potential more than a high accurate model.

Fig. 4.25 shows the potential and the $2 p$ orbital performed by our numerical calculations. As it is expected, there is a structural node along $x$-orientation. This is the node range that we will address in order to apply the QSPIDER lateral momentum-shearing interferometry technique under our 2D-TDSE.
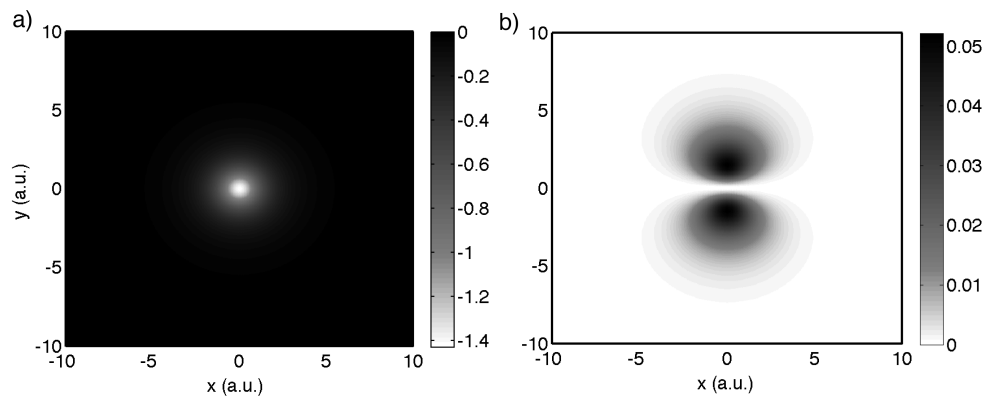

Figure 4.25: Yukawa potential and $2 p$ orbital. a) Depicts the short-range Yukawa potential structure defined by Eq. (4.31) (in gray color scale) as a function of the position $(x, y)$. b) We draw the calculated $2 p$ orbital oriented along $y$-axis. This electron density was calculated by time-imaginary propagation with the parameters described in the text.

Note that (computationally speaking) the calculation of the scattering waves for a 2D plane is arduous. Particularly, in our case where the emitted EWP by the XUV achieves velocities of around 1.5 or 2.0 a.u. This means that we need a large numerical grid which translates in a large number of points $N_{x}$ and $N_{y}$.

For this reason, here we will perform our numerical calculations of the dipole phase utilizing the PW.

\section{Attosecond EWP emission from a short-range potential}

A way to compute the exact EWP amplitude and phase is to integrate numerically the TDSE for the XUV ionization. In order to extract the EWP phase or dipole phase for the photoemission from the $2 p$ orbital, we show 2D-TDSE calculations.

The XUV parameters used in such calculation are: peak intensity $I_{\mathrm{X}}=5.0 \times 10^{12}$ $\mathrm{W} / \mathrm{cm}^{2}$, central frequency $\omega_{\mathrm{X}}=2.0$ a.u., FWHM $\sim 235$ as with and gaussian envelope and $\mathrm{CEP}=\frac{\pi}{2}$. The position-grid parameters are: grid lengths $L_{x}=L_{y}=300$ a.u., with grid steps $\Delta x=\Delta y=0.3$. Meanwhile, the real time step integration is $\Delta t=0.05$ a.u. 
Then we numerically solve the 2D TDSE and remove the bound states by employing a smooth gaussian mask function. Thus, we have obtained the final position-space EWP. The momentum-space representation of the EWP by making a 2D-FFT of the continuum wavefunction, is computed. The resulting electron momentum distribution is depicted in Fig. 4.26a). Two perpendicular nodes are found along the $p_{x^{-}}$and $p_{y^{-}}$ directions. Similar behavior we have observed in the SFA calculations (EWP and dipole amplitude) for the $2 p$ orbital of the $\mathrm{He}^{+}$.
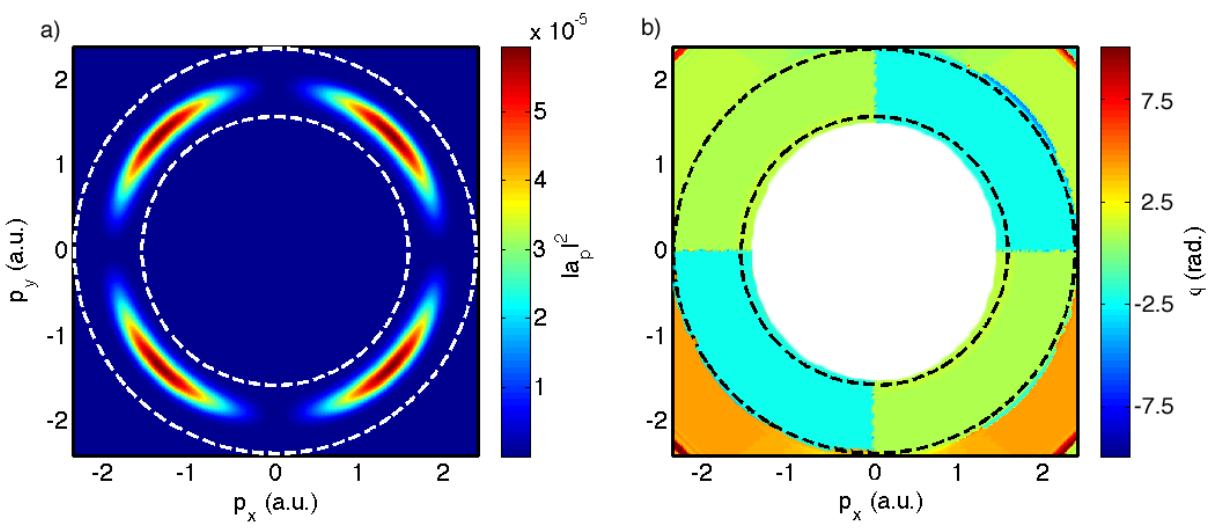

Figure 4.26: EWP amplitude and phase: exact 2D TDSE calculations. Plot a) depicts the final momentum EWP amplitude $\mathrm{S}_{e}(\mathbf{p})=|a(\mathbf{p})|^{2}$ for the ionization driven by a single XUV attosecond pulse from the $2 p$ orbital of a short-range Yukawa potential (see Fig. 4.25b)). The white dashed circles denote the momentum ring around the EWP bandwidth. In the panel b) we show the EWP phase. The circular dashed lines denote the EWP range momentum bandwidth.

The EWP phase or dipole phase is obtained by removing the time-dependent phases given in Eq. (4.2). Fig. 4.26b) shows the result. The black dashed circles denote the momentum bandwidth range of the EWP. Within this region we find a phase jump of $\pi$, depending on which quadrants we look at. Thus, we can consider this phase as a exact reference to be employed for the QSPIDER lateral momentum-shearing interferometry technique.

\section{Lateral momentum-shearing retrieval from a $2 p$ orbital}

We implement the calculation of the lateral momentum-shearing interferograms on the $p_{x^{-}}$and $p_{y^{-}}$-directions. The same pulses scheme for the SFA calculations (see the top plot in Fig. 4.21) are used but here we employ the 2D TDSE calculations. To this end, a large position-space grid will be required. This is so, because, when the first EWP is ionized, it has to be kept inside the calculating position-grid until the second 
EWP is launched to the continuum by the second XUV. Thus, the grid parameters used in such a calculations were: grid lengths $L_{x}=L_{y}=1050$ a.u. with grid steps $\Delta x=\Delta y=0.3$ a.u., for each perpendicular axis $(x, y)$. The real time step is fixed to $\Delta t=0.05$ a.u. The Hamiltonian $H$ of the system which describes the interaction with the atomic electron with the laser fields, is defined by:

$$
H=\frac{1}{2}\left[-i \nabla+\mathbf{A}_{\mathrm{L}}(t)+\mathbf{A}_{\mathrm{X}}\left(t, \tau_{1}, \tau_{2}\right)\right]^{2}+V_{0}(x, y),
$$

where, $\mathbf{p}=-i \nabla=-i\left(\mathbf{e}_{x} \frac{\partial}{\partial x}+\mathbf{e}_{y} \frac{\partial}{\partial y}\right)$ is the momentum operator defined in $2 \mathrm{D}$. The $\mathbf{A}_{\mathbf{L}}(t)$ is the vector potential of the circularly polarized IR laser field and $\mathbf{A}_{\mathbf{X}}\left(t, \tau_{1}, \tau_{2}\right)=$ $\mathbf{A}_{\mathrm{X}}^{(1)}\left(t-\tau_{1}\right)+\mathbf{A}_{\mathrm{X}}^{(2)}\left(t-\tau_{2}\right)$ denotes the vector potential of the APT. The $V_{0}(x, y)$ is the short-range Yukawa potential defined by Eq. (4.31).

The APT parameters used to perform our numerical calculations are similar for both pulses, i.e., the XUV central frequency is $\omega_{\mathrm{X}}=2.0$ a.u., XUV peak intensity $I_{\mathrm{X}}=5.0 \times 10^{12} \mathrm{~W} / \mathrm{cm}^{2}, \mathrm{FWHM} \sim 170$ as under a gaussian envelope and $\mathrm{CEP}= \pm \frac{\pi}{2}$. On the other hand, the IR laser field parameters are: central frequency $\omega_{0}=0.057$ a.u., IR peak intensity $I_{0}=5.0 \times 10^{11} \mathrm{~W} / \mathrm{cm}^{2}$, FWHM $\sim 4$ fs under a sine-squared envelope. The time delay between the two XUV pulses is around $\tau_{\mathrm{x}} \sim T_{0}$. With these parameters, we find that the lateral momentum-shearing on the $y$-direction is $\Delta A_{\mathrm{L}, y}=0.065$ a.u.

Fig. 4.27a) shows the results of the final momentum interferogram with lateral momentum-shearing along the $y$-orientation. We find that the two EWPs interfere around the expected momentum ring, i.e., similar to the behavior shown by the SFA. However, a large additional signal is found around the central momentum which can not come from the emission driven by the XUV attosecond pulses. Otherwise the same signal should be present in Fig. 4.26a). This suggests that the low ionization potential of this $2 p$ state leads to such an ionization signal. This ionization is caused by the circularly IR laser field.

This signal can contain information about the CEP of the IR laser field. Then, this circularly polarized IR laser scheme can be utilized to characterize the CEP of the IR. Nevertheless, this signal is not of our interest in our QSPIDER technique right now. For this reason, we isolate the signal coming from the XUVs and remove the IR ionization. The isolated momentum interferogram signal is depicted in Fig. 4.27b). We also plot a zoom to show the interference fringes around the nodal range on the $p_{x}$-direction. We can observe that the node along $p_{x}$ is upper shifted with respect to $p_{y}=0$. This means that the IR induces appropriately the lateral momentum-shearing 

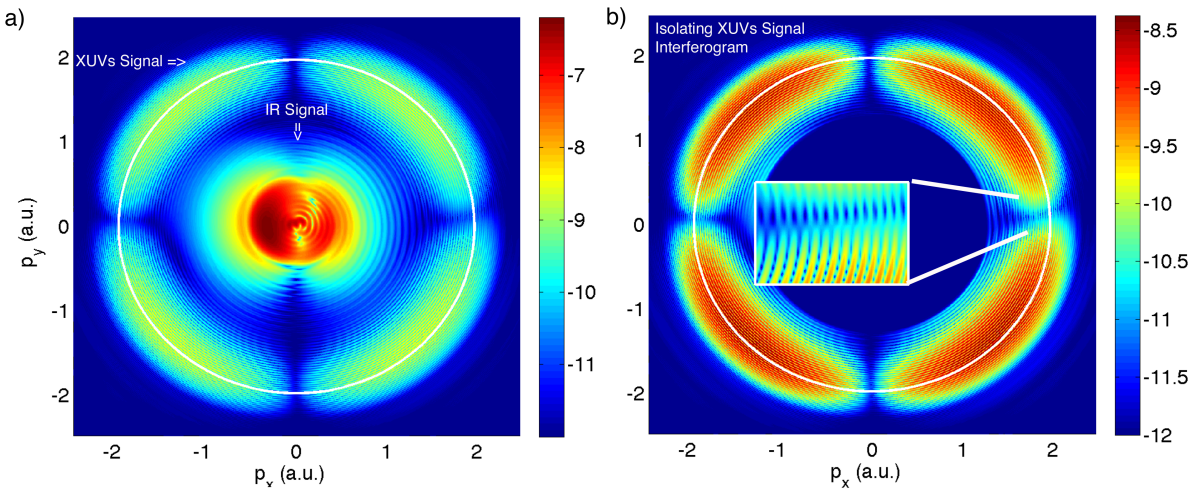

Figure 4.27: EWPs momentum interferogram: 2D TDSE calculations. Plot a) shows the calculated full momentum interferogram for the ionization mediated by the APT and the circularly IR laser fields. b) The isolated interferogram coming from the APT signals. The inset plot depicts a zoom of the interferogram fringes. In both plots, the white circles denote the radial momentum $p_{0}=\sqrt{2\left(\omega_{\mathrm{X}}-I_{p}\right)} \approx 1.9$ a.u.

on the $p_{y}$-direction.

Once we have the first interferogram, which is lateral sheared along $p_{y}$-axis, we calculate the other one, lateral sheared on the $p_{x}$-direction. To do this, we follow the same steps described in Section 4.3.3.1, i.e., we change the time delays $\tau_{1}$ and $\tau_{2}$. From the calculated $p_{x}$ lateral momentum-sheared interferogram, we isolate the XUVs signal as in Fig. 4.27.

So far, we have the two lateral momentum-shearing interferograms and with them we are able to apply the QSPIDER algorithm. The results of the retrieval EWP amplitude and phase gradient are depicted in Fig. 4.28.

The retrieved EWP amplitude is depicted in Fig. 4.28a) and we find out that this amplitude is similar to the one presented in Fig. 4.26a). It is important to point out that an small asymmetry is observed in the full amplitude. This small asymmetry can be related to the influence of the IR laser field at the ionization time.

The EWP phase partial derivatives along the $p_{x}$ and $p_{y}$-directions are plotted in Fig. 4.28b) and c), respectively. Both partial derivatives exhibit two Dirac delta distributions along the expected directions. The partial derivate on the $p_{y}$-axis is a little bit distorted. This is due to the fact that a small deviation around the induced lateral shearing on such $p_{y}$-direction is present. In spite of this small deviation we find a very good agreement with the possible phase gradient of the "EWP phase" depicted in Fig. 4.26b).

Finally, our full 2D TDSE numerical results show that the EWP amplitude and 

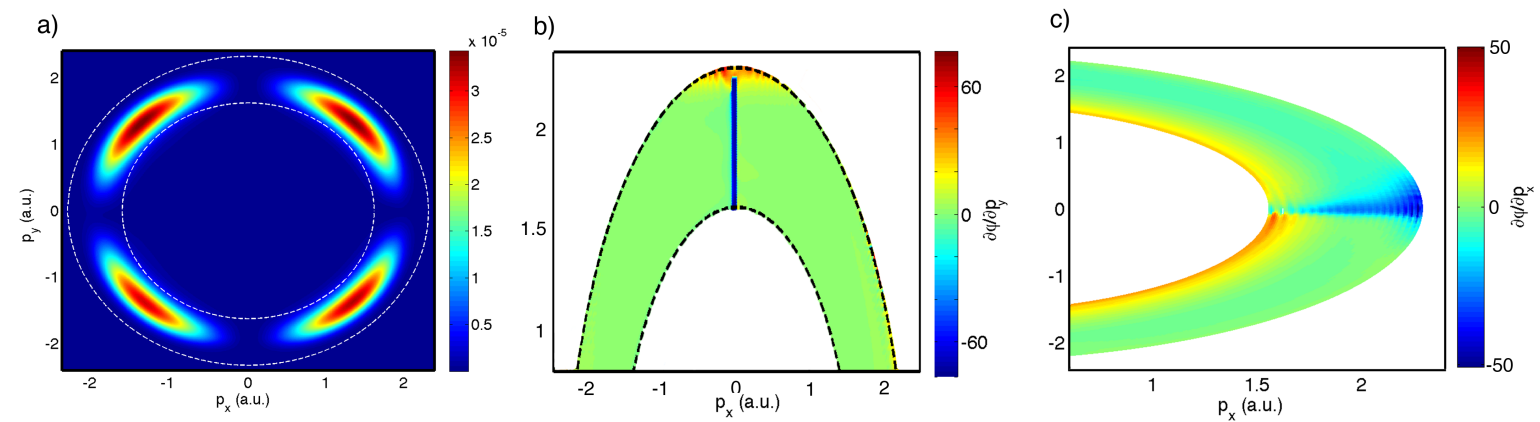

Figure 4.28: QSPIDER for the 2 $p_{y}$ of a Yukawa potential: 2D TDSE model. Plot a) shows the reconstructed EWP amplitude $|a(\mathbf{p})|^{2}$. The QSPIDER retrieval for the dipole phase partial derivatives $\left(\frac{\partial \phi_{d_{2 p}}}{\partial p_{x}}, \frac{\partial \phi_{d_{2 p}}}{\partial p_{y}}\right)$ are depicted in b) and c), respectively.

EWP phase or dipole phase can be reconstructed by utilizing the QSPIDER in the extended version of the lateral momentum-shearing interferometry technique.

\subsection{Conclusions}

In this Chapter we have presented the QSPIDER technique in $1 \mathrm{D}$ and its $2 \mathrm{D}$ version to reconstruct the complex EWP and the complex dipole transition matrix element. The ionization driven by an APT of two pulses in the presence of a weak IR laser, results in an interferogram pattern.

This interferogram codes full information about the phase difference of a copy of the original EWP. If the pulses are well characterized, our numerical results under the full TDSE in 1D and 2D as well as the SFA model show that the EWP amplitude and phase can be reconstructed. It is demonstrated that this technique is able to extract the complex dipole within the EWP momentum-bandwidth as well.

QSPIDER inherits most of the known issues of the SPIDER techniques. As a consequence, high resolution is needed to measure the interferogram. In the case of QSPIDER this is related to the energy or momentum resolution in the spectrometer. Several methods have been proposed to overcome these issues for SPIDER which may be helpful also in the QSPIDER scheme [150, 154].

Note that the two versions of QSPIDER have interesting desirable features from an experimental viewpoint. First, the subsequent emission of two EWPs by the application of the two colors, i.e., the attosecond pulse train of two pulses and the IR laser, to the target, shows that the two versions of QSPIDER are single shot techniques. Second, 
the interaction of the two attosecond pulses with the same ground state suggests that QSPIDER is an auto-referenced technique.

Furthermore, we suggest to apply the QSPIDER technique in future experimental setups in order to extract dynamical information of the photoemission process. It is, to study the Wigner time delay without the need of a reference orbital or state. 
CONCLUSIONS: CHAPTER 4 
5

\section{Delay in photoemission}

\subsection{Introduction}

When the XUV photon energy of a SAP or an APT is larger that the ionization potential of an atom or a molecule, the electron can be ionized. This process creates a coherent ultra broadband EWP and the momentum distribution of the ionized electrons maps both the characteristics of the attosecond pulses and the parent system [61, 62, 67].

Another intriguing property is that this electron is not emitted instantaneously. Instead, the atom or molecule may have a response time or "delay" in the photoemission process [68]. This delay is related to the so-called Wigner time [69, 70] which measures the travel time difference between a free electron and an electron under the influence of a short-range potential. Formally, the Wigner time is defined as the derivative in energy of the bound-free dipole transition matrix element ${ }^{1}$ phase or the phase shift that takes the EWP in the one-photon ionization process [69, 70, 71].

The response time of the atom or molecule is then encoded in the phase of the EWP and provides valuable information about the system [67,68]. Considering this information is encoded in the phase, traditional observables cannot access it.

Only recently some experiments related to the delay in photoinization have been carried out, thanks to the now available attoscience tools. Schultze and coworkers [17] have measured the relative delay in photoemission from the $2 s$ and $2 p$ states of $\mathrm{Ne}$ atoms using the streaking technique [37]. Also recently, the RABBIT technique [38] has been used to measure the relative time delay between the $3 s$ and $3 p$ states in $\operatorname{Ar}$ [18].

Recent works have also theoretically addressed the Wigner time delay in small

\footnotetext{
${ }^{1}$ For simplicity, in this Chapter we will refer to the bound-free dipole transition matrix element as dipole.
} 


\section{INTRODUCTION}

molecules, hydrogen molecules [75, 76], emphasizing the consequences of having two centers. In reference [76] they have been also studied the dependence of the Wigner time delay on the internuclear distance. It is also addressed the angular dependence of the time delay for the hydrogen molecular ion.

These results show that the Wigner time delay also contains information about the geometrical structure of the molecule. Most importantly, the observable streaking time delay, can be extracted and compared to the intrinsic Wigner time. However, it is important to note that the Coulomb-laser coupling can not be removed from the measurement process.

Also those studies described in the introductory Section 1.4 about the influence of the IR laser field on the detection of the Wigner time have shown that the CLC plays an important role in the measurement process.

In this Chapter we study the Wigner time delay, its problematic measurement by the pump-probe streaking technique and, most importantly, we suggest a new way to remove the CLC from the streaking measurement process.

We address the open questions about the Wigner time which have been described in Sections 1.4 and 1.5. To this end, we propose a small asymmetric molecular system and define an alternative quantity which is named the Stereo Wigner Time Delay. We will show that by the measurement of two attosecond streaking traces, one on the left and another one on the right side of the electron emission, and by the comparison of the extracted streaking time delay, the symmetric CLC influence is removed.

The organization of this Chapter is done in three main Sections 5.2, 5.3 and 5.4. In the Section 5.2, we introduce the Wigner time delay concept: the quantum picture and the classical one. In the following Sections 5.2.1 and 5.2.1, we will study how the Wigner time depends on the potential structures, i.e., the short-range potential and long-range potential for atomic systems. The classical picture and the quantum definition are compared to both kinds of potentials. In the next Section 5.2.2, the study of how the delay in photoemission can be linked to the Wigner time by utilizing the attosecond streaking technique will be carried out. We will use as test potentials the Yukawa potential and Coulomb potential. Detail information about how to extract the delay in photoemission from the streaking traces is also provided.

The next Section 5.3, will be dedicated to propose an alternative small asymmetric molecular system to study the SWTD, i.e., the CO potential. The definition of the molecular system, the dipole structure, the Wigner time and the asymmetric in the Wigner time will be treated with more details in the Sections 5.3.1 and 5.3.2.

The detection method of the Wigner time such as the Stereo Time Of Flight (STOF) 
and its comparison with the left-right energy derivative of the dipole phase will be defined in Section 5.3.3. More importantly, we will present our complementary method, i.e., the stereo streaking technique, about how to extract directly for first time the SWTD in the photoemission process in the Section 5.3.4.

In the Section 5.4, we propose a "simple" asymmetric 3D molecular model to extend our 1D calculations about the SWTD. In the following Section 5.4.2, we re-define the stereo time of flight method in the 3D case, as an alternative to obtain the SWTD. The stereo streaking technique, its problems and solutions to recover the SWTD from the asymmetric molecule will be studied in the Section 5.4.3.

In the Section 5.4.3.1, we will show that if the streak IR laser field couples two bound orbitals, the streaking measurement carries out information about the electron dynamics between the two coherent states.

Finally, we will present our conclusions and outlooks in the Section 5.5.

\subsection{Wigner time in atoms}

In this Section we introduce the quantum and classical pictures of the Winger time delay considering that the photoionization of an atomic system is driven by a XUV SAP.

When an EWP is released as result of the absorption of a SAP by an atomic system, a time delay in the ionization process takes place. This time delay is defined as the time elapsed between the arrival of the SAP and the actual time when the EWP is emitted. It is the so-called delay in photoemission which is close to the Wigner time delay.

This Wigner time is defined by the energy derivative of the dipole phase or the scattering wave phase-shift, $\phi=\phi_{l}(k)$, accumulated by the EWP during the ionization process $[17,69,72]$ :

$$
\Delta t_{\mathrm{W}}=\frac{\partial \phi}{\partial E}
$$

where $\phi$ is the phase-shift of the dipole phase of the emitted EWP with energy $E=\frac{k^{2}}{2}$ with asymptotic momentum $k$. In general, the phase-shift will depend on the linear momentum $k$ and the quantum angular momentum $l$.

The first interpretation of the energy derivative of the phase shift as a time delay was proposed by Eisenbud [71] and Wigner [69]. Wigner showed that the time delay between 
an incident wavepacket to a short-range scattering center and the outgoing wavepacket is related to the energy derivative of the quantum phase-shift. This derivation was performed in order to address the question if a scattered wave can (or can not) leave the scatter center before the incident wave reaches it [69] as it is illustrated in Fig. 5.1.

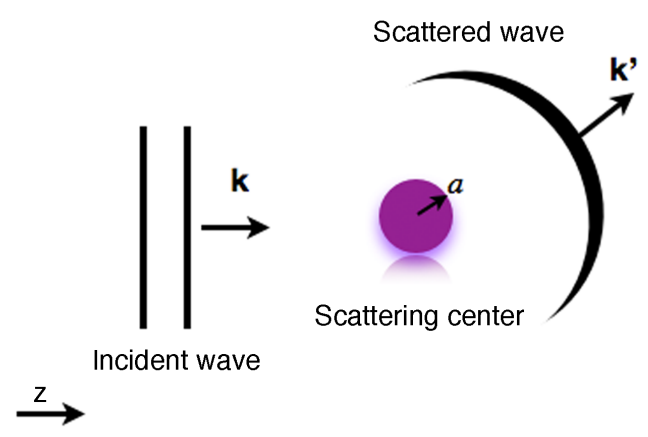

Figure 5.1: Conceptual Wigner Time. Scheme of the delay between the incident wave of momentum $\mathbf{k}$ to a scattering center of radius $a$ and the scattered wave of momentum $\mathbf{k}^{\prime}$.

In the same paper, Wigner showed that $\Delta t_{\mathrm{W}}>0$ only in the case of attractive potentials and for a near-resonance energy or $\Delta t_{\mathrm{W}}<0$ for the rest of the energy values (in case of a short-range potential of radius $a$ ) [75].

A classical picture of the Wigner time can also be obtained by the integration of Newton's equations for the time of flight that an electron, with "asymptotic momentum" $\mathbf{k}$, spends under the action of a binding potential $V_{0}(\mathbf{r})$ with respect to the free motion:

$$
\Delta t_{\mathrm{Cl}}=\int_{\mathbf{r}_{0}}^{\mathbf{r}_{f}} \frac{d r}{\pi\left(\mathbf{r}, E_{k}\right)}-\Delta t_{\mathrm{Free}}
$$

where $^{1} \pi\left(\mathbf{r}, E_{k}\right)=\sqrt{2\left(E_{k}-V_{0}(\mathbf{r})\right)}$ is the local momentum [68] of the electron as a function of the electron trajectory $\mathbf{r}=\mathbf{r}(t)$ and the total energy $E_{k}=\frac{k^{2}}{2}, \mathbf{r}_{0}$ and $\mathbf{r}_{f}$ are the initial and final positions, and the $\Delta t_{\text {Free }}=\frac{\left|\mathbf{r}_{f}-\mathbf{r}_{0}\right|}{k}$ is the time that a free electron spends between both positions.

In order to figure out what it is the influence of a short-range or a long-range potentials in the Wigner time delay, we choose the Yukawa and the soft-core hydrogenic Coulomb potentials in order to "test" the scattering centers. These scattering centers will allow us to compare the effects of the short- and long-range potentials in the Wigner time.

\footnotetext{
${ }^{1}$ Here, $\pi$, also denotes the module or norm of the kinetic momentum $\pi$ and no the number 3.1416.
} 
For any arbitrary system in one dimension (under the SAE approximation), it is easy to compute the phase shift from the bound-free dipole transition matrix element. This dipole is computed by the described scattering waves in Section 2.2.1.4 of the Chapter 2.

\subsubsection{Wigner time by quantum and classical pictures}

The Wigner time is the energy derivate of the dipole phase. This phase is a structural feature of the atomic or molecular system. This Section is dedicated to the study the Wigner time in the short- and long-range potential. As test atomic systems, we choose the Yukawa potential and Coulomb potential.

For simplicity, we restrict ourself to the 1D models. This restriction is well based on the fact that linearly polarized laser pulses are utilized in our description of the Wigner time. In such a case, the electron mainly will follow the direction of the laser polarization. In other words, any magnetic quantum momentum is excited for the electron wavefunction.

In the next, we introduce the potentials, and compute the structural bound states, the complex dipoles and the Wigner time delays in case of the quantum and classical pictures.

\section{Definition of the system}

The field-free Hamiltonian $H=\frac{p^{2}}{2}+V_{0}(z)$ defines the discrete energy spectrum, or bound states, as well as the continuum spectrum of a particular potential $V_{0}(z)$. To study the Winger time of an emitted EWP, we use the Yukawa $V_{0, Y}(z)$ and soft-core Coulomb $V_{0, \mathrm{C}}(z)$ potentials:

$$
\begin{aligned}
V_{0, Y}(z) & =-Z \frac{e^{-\frac{r^{\prime}(z)}{a_{s}}}}{r^{\prime}(z)} \\
V_{0, \mathrm{C}}(z) & =-\frac{Z}{r^{\prime}(z)},
\end{aligned}
$$

where $r^{\prime}(z)$ is defined by, $r^{\prime}(z)=\sqrt{a+z^{2}}$, and, $a$, is the soft-core parameter, $a_{s}$, the screening charge parameter and, $Z$, the total charge. These parameters have been chosen in such a way to match the ground state energy, $E_{0, \mathrm{H}}=-0.5$ a.u., of the hydrogen atom. Specifically $Z=1, a=0.5$ and $a_{s}=2$ a.u., for the Yukawa potential and $Z=1, a=2.0$ a.u., for the soft-core Coulomb potential, respectively. For simplicity 
we will refer to the soft-core Coulomb as Coulomb potential.

The ground wave functions $\left(\Psi_{0, Y}(z)\right.$ and $\left.\Psi_{0, \mathrm{C}}(z)\right)$ are calculated by imaginary time propagation with a time step of $\Delta t=-0.01 i$ a.u. The grid parameters are $\Delta z=0.1$ a.u., and with a spatial grid length of 2500 a.u.

Fig. 5.2 depicts the potentials as well as the electron densities, $\left|\Psi_{0, \mathrm{Y} / \mathrm{C}}(z)\right|^{2}$, of the ground states for the short-range Yukawa and long-range soft-core Coulomb potentials as a function of the position $z$, respectively. The comparison between both potentials shows that the short-range potential goes to zero much faster compared to the longrange Coulomb potential. In addition, the ground states have different shapes, i.e., the electron density for the ground state of the Coulomb potential is wider that the Yukawa potential one.

a)

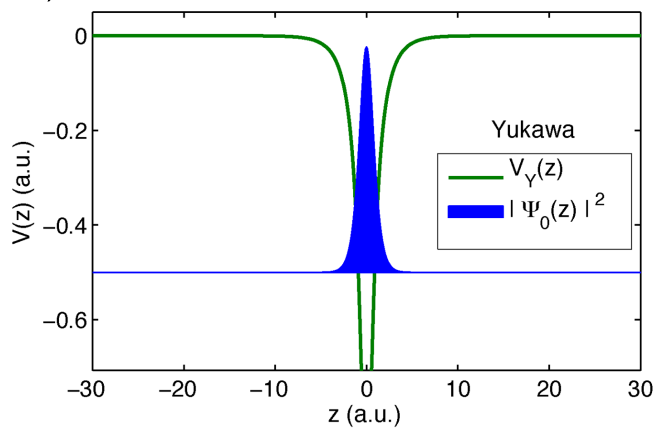

b)

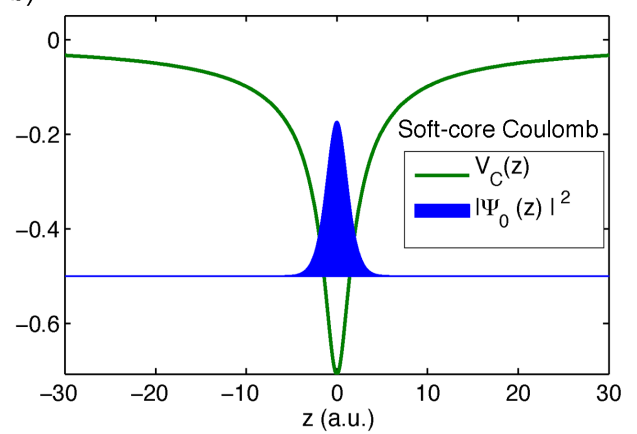

Figure 5.2: 1D Yukawa, Coulomb potentials and ground states. a-b) The scattering center (green dark lines) for the short-range Yukawa $V_{0, \mathrm{Y}}(z)$ and long-range Coulomb $V_{0, \mathrm{C}}(z)$ potentials. The electron densities $\left|\Psi_{0, \mathrm{Y} / \mathrm{C}}(z)\right|^{2}$ of the ground states (blue area) for both potential systems are also shown.

To extract the exact quantum Wigner time delay related to the ground states of the Yukawa and Coulomb potentials, we compute the amplitude and phase of the complex dipole transition matrix element bound-free $d(k)=-\int d z \Psi_{k}^{*}(z) z \Psi_{0}(z)$. We consider the continuum state of momentum $k$ as a $\mathrm{PW} \Psi_{k}(z)=\frac{1}{\sqrt{2 \pi}} e^{i k z}$ or as a SW $\Psi_{k}(z)$ (explained in the Section 2.2.1.4 of Chapter 2). Then, we evaluate the energy derivative of the dipole phase $\Delta t_{\mathrm{W}}=\frac{1}{k} \frac{\partial \phi(k)}{\partial k}$ and compare it to the classical Wigner time delay $\Delta t_{\mathrm{Cl}}$ of our 1D models. The momentum grid parameters used are $\Delta k=0.005$ a.u. and the momentum grid length 10 a.u.

Fig. 5.3 shows the amplitude $|\mathrm{d}(k)|$ and phase $\phi(k)$ of the dipole transition matrix element for the Yukawa and Coulomb potentials by utilizing PWs and SWs. 
Besides of the fact that the absolute dipole amplitudes are different, they have similar structures when both, i.e., plane and scattering waves, are used to compute the complex dipole and for both systems $V_{0, Y}$ and $V_{0, \mathrm{C}}$. In contrast, the dipole phases are completely different.

In case of $\mathrm{PW}$, the dipole phase is constant for negative $k<0$ or positive momenta $k>0$ just with a phase jump of $\pi$ at $k=0$, but in case of SW the dipole phase is not constant. Then, we can deduct that the energy derivative of the dipole phase is zero for the PW (except to $k=0$ ) while in case of the SW it is different from zero in all the momentum range depicted in Fig. 5.3). This indicates that specific structural information related to the dipole phase $\phi(k)$ is obtained by the SW whereas in the $\mathrm{PW}$ this is not the case.
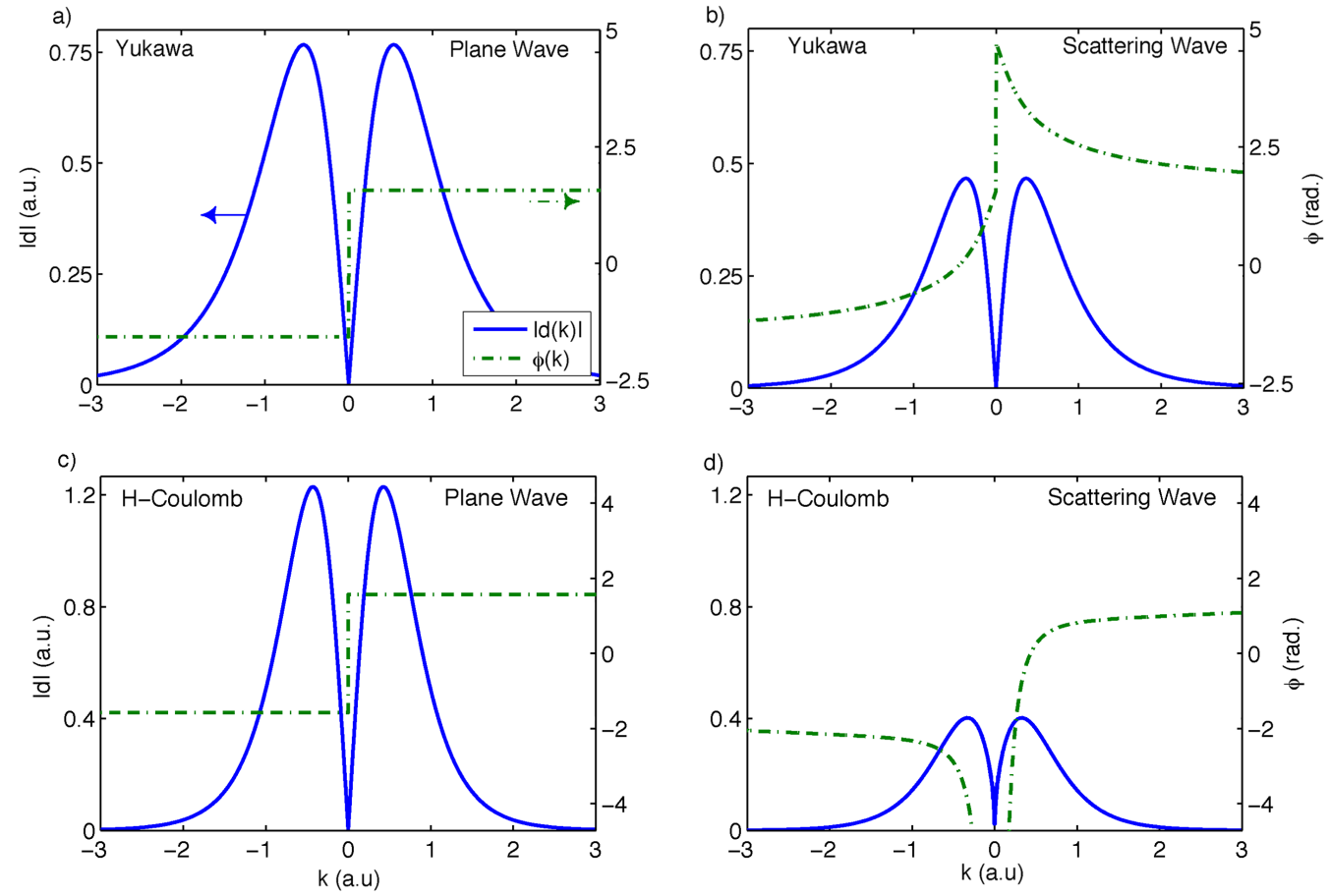

Figure 5.3: Dipole matrix element structure for Yukawa and Coulomb potentials. a) and b) show the amplitude $|d(k)|$ (blue line) and phase (short-range part) $\phi(k)$ of the dipole as a function of the asymptotic momentum $k$ for 1D Yukawa short-range potential by projecting on PWs, and SWs, respectively. c-d) The same as in a-b) but for a 1D H Coulomb potential

The quantum picture of the Wigner time delay is based on the energy derivative of the short-range dipole phase term or phase-shift [72] and for this reason, the use of SW 
to compute the dipole phase is of mandatory importance for both system.

The dipole phases in the Yukawa and Coulomb potentials are the same when PWs (see Fig. 5.3a-c)) are used to compute the dipole. However, they strongly differ in case that SWs are employed in the calculation (see Fig. 5.3b-d)). This reflects also the difference of the detailed information that the Yukawa and Coulomb potentials encode in the SW when the complex dipole is computed.

The Fig. 5.4 shows the Wigner time delay obtained by the quantum treatment Eq. (5.1) and by classical definitions Eq. (5.2). For the classical calculations the initial position $z_{0}$ is set to zero and the final one is $z_{f}$ (maximum of the spatial position grid $z_{f}=$ $N_{z} \Delta z / 2$ ). The quantum and classical time delays are in very good agreement in case of the Yukawa potential (see Fig. 5.4a)). In clear contrast, the quantum picture differs strongly from the classical one in the case of the Coulomb potential (see Fig. 5.4b)).
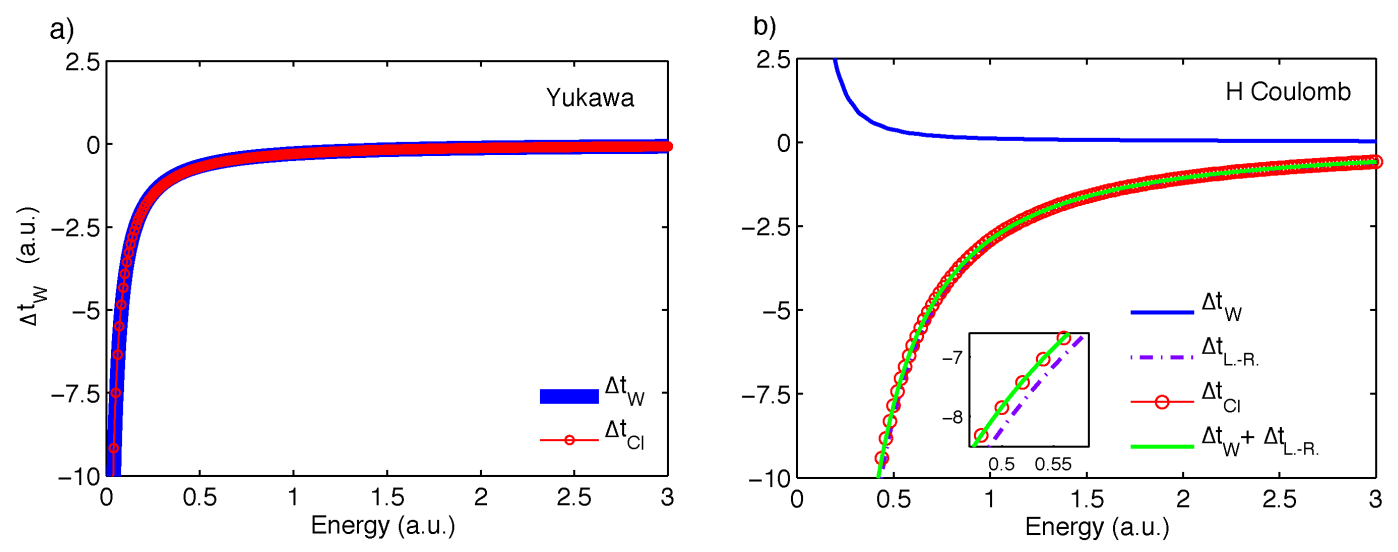

Figure 5.4: Quantum and classical pictures of the Wigner time delay. a-b) The quantum (blue line) and classical (red line and circles) pictures of the Wigner time delay are depicted for the short-range Yukawa and long-range Coulomb potentials, respectively. In b) the violet dashed line with points is the long-range time delay contribution from the Coulomb wave phase. Green line shows the total time delay $\Delta t_{\text {Tot }}$ which is defined by the sum of Wigner time delay and the long-range delay $\Delta t_{\mathrm{Tot}}=\Delta t_{\mathrm{L}-\mathrm{R} \text {. }}+\Delta t_{\mathrm{W}}$. Inset graph depicts a zoom within a small energy range which compares the total quantum time delay $\Delta t_{\text {Tot }}$ and the classical one $\Delta t_{\mathrm{Cl}}$.

This disagreement is due to the quantum definition of the Wigner time delay which takes into account only the phase-shift, $\phi(k)$, (or short-range phase term) energy derivative of the continuum scattering Coulomb wave $[69,68,72,155,156]$. This definition ignores the long-range phase term, $\phi_{\mathrm{L} .-\mathrm{R} .}(k)=\frac{Z}{k} \ln \left|2 k z_{f}\right|$, of the Coulomb wave [68, 75].

That logarithmic phase is as a function of the final position $z_{f}$ and the asymptotic 
momentum $k$. In order to obtain the long-range time delay contribution, we compute the energy derivative $\Delta t_{\mathrm{L} .-\mathrm{R} .}=\frac{\partial \phi_{\mathrm{L} .-\mathrm{R}} \text {. }}{\partial E}$ of the long-range phase term, which can be written as:

$$
\Delta t_{\mathrm{L} .-\mathrm{R} .}(E)=\frac{1}{\sqrt{(2 E)^{3}}}\left(1-\ln \left[2 \sqrt{2 E} z_{f}\right]\right)
$$

For an $\mathrm{H}$ Coulomb potential, $z_{f}$ is fixed to the electron position at the end of our numerical spatial grid.

We show in Fig. 5.4b) this long-range time delay term dominates over any short-range Wigner time delay $\Delta t_{\mathrm{W}}$ for the Coulomb potential case. We have added up the longrange time delay contribution $\Delta t_{\mathrm{L} . \mathrm{R} \text {. }}$ to the Wigner time delay $\Delta t_{\mathrm{W}}$ and we find that this total time delay, $\Delta t_{\text {Tot }}$, is in very good agreement with the classical calculations $\Delta t_{\mathrm{Cl}}$.

A detail analysis about how to link the Wigner time with the delay in photoemission is provided in Sections A.1-A.4 of Appendix A.

Note that this long-range time delay contribution diverges logarithmically as $z_{f}$ increases and it cannot be ignored. This means that any absolute time delay cannot be well defined in the sense that there is no convergence limit in $\Delta t_{\mathrm{L} .-\mathrm{R}}$. when $z_{f} \rightarrow \infty$ $[68,157]$.

In this Section, we have analyzed the quantum and classical pictures of the Wigner time delay for the short-range Yukawa and long-range Coulomb potentials. Our analysis shows that quantum definition of the Wigner time delay can be perfectly linked to the classical one in case of the short-range Yukawa potential. However, in the case of the long-range Coulomb potential an extra time delay needs to be added up to the Wigner time delay in order to find a perfect agreement between the quantum and the classical pictures.

In the next Sections we will address in details the study of how the delay in photoemission can be linked to the Wigner time delay utilizing the attosecond streaking technique. We will use as test potentials the Yukawa and Coulomb potential systems.

\subsubsection{Measurement of Wigner time by streaking techniques}

Attosecond streaking has become one of the most important tools of attosecond science. The attosecond streaking is a variant of a pump-probe technique where a SAP is the pump which releases an EWP from the system, and a few-cycle IR laser streaks, i.e., probes, the EWP when in the continuum [3]. 
If the probe laser has a weak intensity and the XUV attosecond time duration is much shorter than one IR laser cycle $T_{0}$, the final electron momentum $p_{f}$ is modified according to the equation:

$$
p_{f}(\tau)=p_{0}-A_{\mathrm{L}}(\tau)
$$

where $\tau$ is the time delay between the pump XUV and the probe IR laser fields. $A_{\mathrm{L}}(\tau)$ is the vector potential of the IR laser pulse at $\tau$. Here, $p_{0}$, is the asymptotic momentum related to the photoelectron energy, $E_{0}=\frac{p_{0}^{2}}{2}$, which depends on the central XUV frequency $\omega_{\mathrm{X}}$ and the ionization potential ${ }^{1} I_{p}$. The time delay in photoemission or Wigner time delay $\Delta t_{\mathrm{W}}$ is intuitively expected to shift the appearance of the EWP at the time $\tau$ (see Fig. 5.6). According to Nagele et al. [72] the electron motion is distorted, classically, by the action of the potential, then the modified final momentum Eq. (5.6) becomes:

$$
p_{f}(\tau) \approx p_{0}-\alpha A_{\mathrm{L}}\left(\tau+\Delta t_{\mathrm{S}}\right)
$$

where $\alpha$ is a correction factor for the amplitude of the momentum shift induced by the laser field [158], and $\Delta t_{\mathrm{S}}$ is the streaking time delay in the photoemission which includes information about the Wigner time delay of the photoionization process [17, 72].

The sign convention for $\Delta t_{\mathrm{S}}$ ensures that positive (negative) values correspond to delayed (advanced) emission with respect to maximum of the XUV envelope centered at $\tau$ as it is illustrated in Fig. 5.5. This positive values of $\Delta t_{\mathrm{S}}$ means the electron "feels" the IR vector potential after than $\tau$.

The blue (green) point in Fig. 5.5 marks the value of the vector potential that the electron will feel if it is ionized before (after) the arrival of the XUV attosecond pulse.

From the attosecond streaking trace, we will recover the streaking time shift $\Delta t_{\mathrm{S}}$. The streaking spectrogram is the construction of the electron momentum distribution as a function of the time delay between the IR and the XUV laser pulses. The momentum expectation value, $\left\langle p_{\mathrm{R}}\right\rangle$, is computed as a function of the time delay $\tau$. Thus, the attosecond streaking time, $\Delta t_{\mathrm{S}}$, is extracted by Fourier analysis of this momentum expectation value. This method will be explained in detail below.

The attosecond streaking trace is numerically computed by solving TDSE un-

\footnotetext{
${ }^{1}$ Einstein's formula for photoelectric effect $E_{0}=\omega_{\mathrm{X}}-I_{p}$
} 


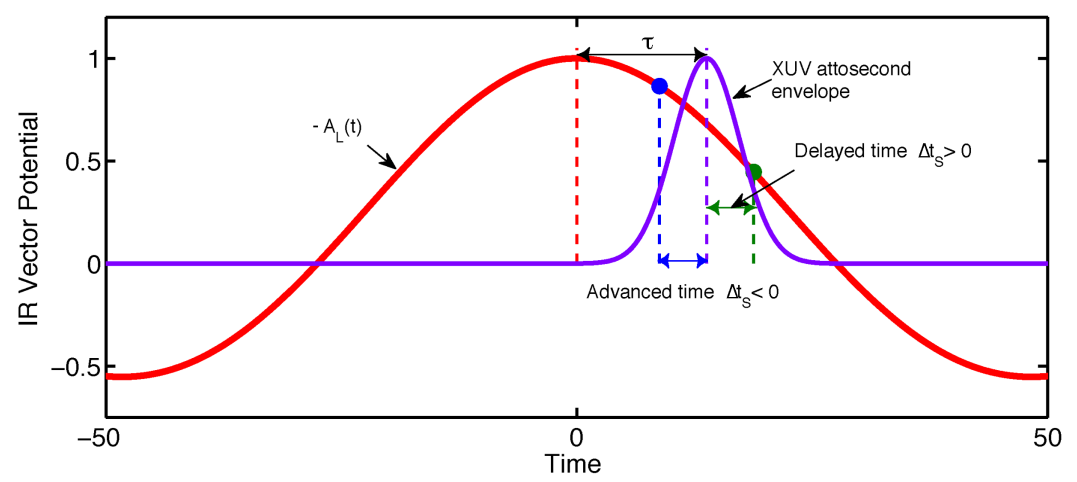

Figure 5.5: Advanced and delayed streaking time scheme. The red and violet lines depict the IR vector potential $-A_{\mathrm{L}}(t)$ and the XUV attosecond pulse envelope, respectively. The delay between the IR and the XUV laser pulses is denoted by $\tau$ and it is illustrated by the time between the red dashed vertical and violet dashed vertical lines. If the electron is ionized after (before) of the XUV attosecond maximum this corresponds to the delayed (advanced) emission, which means that $\Delta t_{\mathrm{S}}>0\left(\Delta t_{\mathrm{S}}<0\right)$.

der the streaking conditions. As it is explained above, these conditions have to ensure that the time duration of the $\operatorname{SAP} E_{\mathrm{X}}(t-\tau)$ have to be much shorter than an IR cycle $T_{\mathrm{L}}$ and the few-cycle IR laser pulse $E_{\mathrm{L}}(t)$ has to have a moderated intensity $\left(I_{0}=10^{11}-10^{12} \mathrm{~W} / \mathrm{cm}^{2}\right)$. We choose the ground-states of the short-range Yukawa and long-range Coulomb potentials as the test systems. The Hamiltonian $H=\frac{1}{2}\left[p+A_{\mathrm{X}}(t-\tau)+A_{\mathrm{L}}(t)\right]^{2}+V_{0, \mathrm{Y} / \mathrm{C}}(z)$ describes the interactions of the system in the velocity gauge and $A_{\mathrm{L}}(t)=-\int^{t} E_{\mathrm{L}}\left(t^{\prime}\right) d t^{\prime}$ is the vector potential of the IR laser pulse.

The continuum wave function part, $\Psi_{c}\left(z, \tau, t_{f}\right)$, of the whole wavefunction $\Psi\left(z, \tau, t_{f}\right)$ is calculated by masking all the possible bound states of $\Psi\left(z, \tau, t_{f}\right)$ at the final time $t_{f}$. The final momentum electron distribution $\left|\Psi_{c}\left(p, \tau, t_{f}\right)\right|^{2}$ is built by projecting $\Psi_{c}\left(z, \tau, t_{f}\right)$ on $\mathrm{PW}$ or SW. For simplicity, below we will cite the wave function $\Psi_{c}\left(z, \tau, t_{f}\right)$ as $\Psi_{c}(z, \tau)$.

To extract the time delay in photoemission from the streaking trace, we calculate the momentum expectation value on the right ${ }^{1}\left\langle p_{\mathrm{R}}\right\rangle=\frac{\int_{0}^{\infty} p\left|\Psi_{c}(p, \tau)\right|^{2} d p}{\int_{0}^{\infty}\left|\Psi_{c}(p, \tau)\right|^{2} d p}$ as a function of the XUV-IR time delay $\tau$ and then compare it to the IR vector potential $-A_{\mathrm{L}}(\tau)$. To do this comparison, we compute a Fourier transform $\langle\tilde{p}\rangle=\int_{-\infty}^{\infty} d \tau\langle p(\tau)\rangle e^{-i \omega \tau}$ of the momentum expectation value. The spectral phase $\phi(\omega)$ of $\langle\tilde{p}\rangle$ is related to the time shift $\Delta t_{\mathrm{S}}$ between the vector potential of the IR pulse and the streaking momentum expectation value.

\footnotetext{
${ }^{1}$ In order to simplify our notation we will change $\left\langle p_{\mathrm{R}}\right\rangle$ by $\langle p\rangle$.
} 

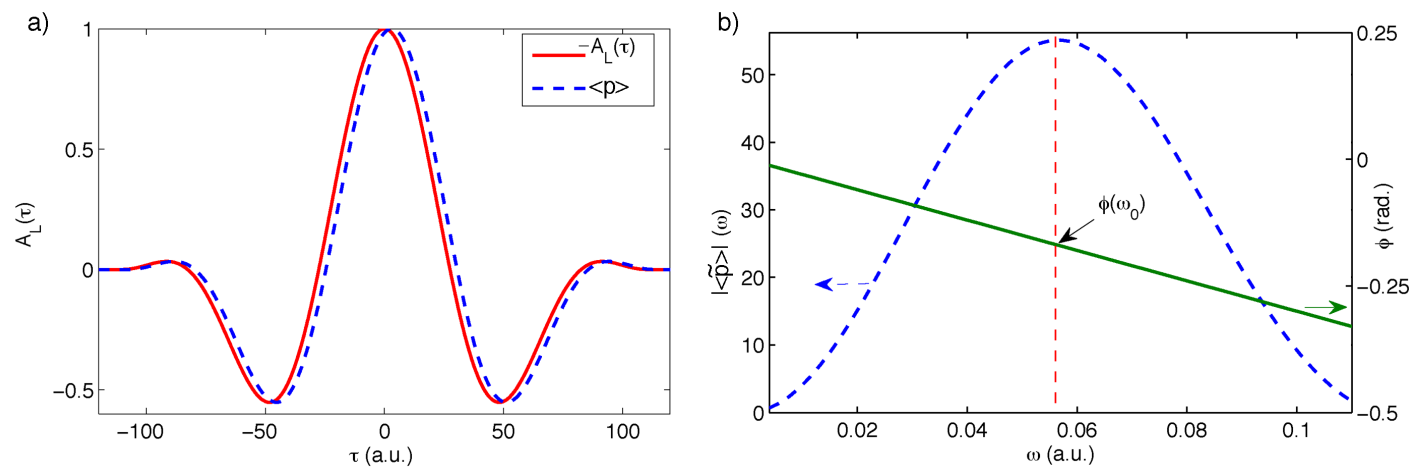

Figure 5.6: Fourier analysis for the extraction of the streaking time delay. a) The vector potential of the IR laser pulse $-A_{\mathrm{L}}(\tau)$ (red line) and the momentum expectation value $\langle p\rangle$ (blue dashed line) as a function of the time delay $\tau$. b) Spectral amplitude (blue dashed line) and spectral phase (green line) of $\langle\tilde{p}\rangle$. The ratio $\Delta t_{\mathrm{S}, \text { point }}=\phi\left(\omega_{0}\right) / \omega_{0}$ or $\Delta t_{\mathrm{S} \text {, derv }}=$ $\left.\frac{d \phi(\omega)}{d \omega}\right|_{\omega=\omega_{0}}$ gives the time delay between $-A_{\mathrm{L}}(\tau)$ and $\langle p\rangle$.

Fig. 5.6 illustrates a scheme of the basic procedure to extract the streaking time delay in photoemission $\Delta t_{\mathrm{S}}$. A clear time shift between the vector potential $-A_{\mathrm{L}}(\tau)$ of the IR laser and the momentum expectation $\langle p\rangle$ is observed in Fig. 5.6a). Furthermore, the amplitude and phase of $\langle\tilde{p}\rangle$ is depicted in Fig. 5.6b). The ratio $\Delta t_{\text {S,point }}=\phi\left(\omega_{0}\right) / \omega_{0}$ or the derivative of the spectral phase evaluated at $\omega_{0}\left(\Delta t_{\mathrm{S}, \mathrm{derv}}=\left.\frac{d \phi(\omega)}{d \omega}\right|_{\omega=\omega_{0}}\right)$, gives the time delay $\Delta t_{\mathrm{S}}$ between $-A_{\mathrm{L}}(\tau)$ and $\langle p\rangle$, where $\omega_{0}$ is the central frequency of the vector potential $A_{\mathrm{L}}(\tau)$.

Figs. 5.7 and 5.8 show the streaking traces calculated by the TDSE and using PW and SW for the cases of Yukawa and Coulomb potentials. The attosecond streaking traces calculated by PW and SW are different only for the maxima values of the photoelectron momentum distributions. The momentum expectation values (PW and SW) depend whether the short-range Yukawa or the long-range Coulomb potentials are employed.

The momentum expectation value $\left\langle p_{\mathrm{R}}\right\rangle$ as a function of the time delay $\tau$ is depicted in Fig. 5.7 and 5.8a-b) for both potentials and by employing PW and SW.

The same result is obtained for the momentum expectation value computed by PW or SW projections in case of a Yukawa potential as it is expected. In the Coulomb potential, on the other hand, the momentum expectation values obtained from $\mathrm{PW}$ or SW are slightly different.

The comparison between the vector potential $-A_{\mathrm{L}}(\tau)$ and the shifted expectation value $\left\langle p_{\mathrm{R}}^{\prime}\right\rangle=\left\langle p_{\mathrm{R}}\right\rangle-p_{0}$ shows a clear time shift $\Delta t_{\mathrm{S}}$ (see the inset graphs in Fig. 5.7 and $5.8 \mathrm{c}-\mathrm{d})$ ). We extract this time shift from the momentum expectation value (PW and 

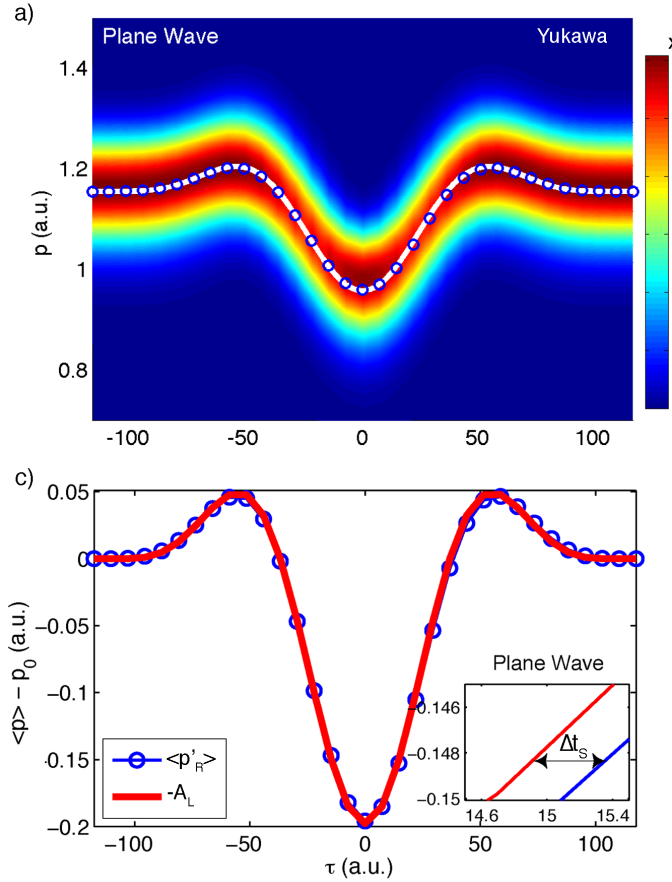

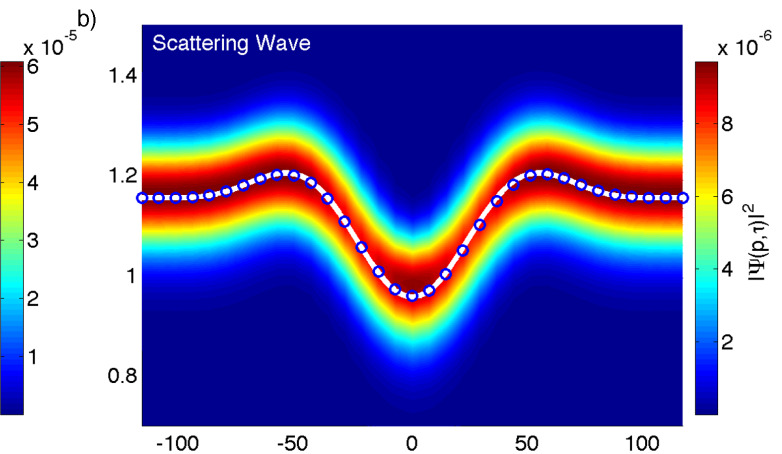

d)

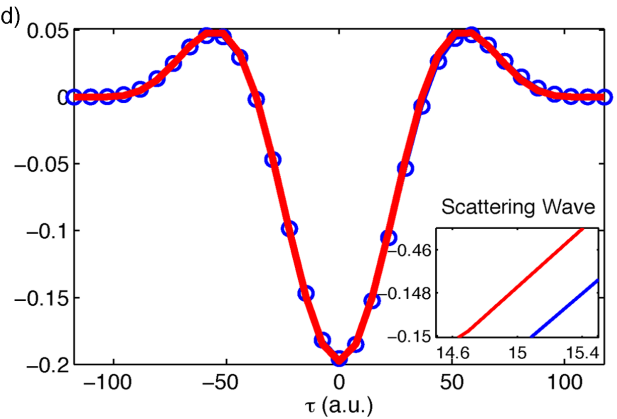

Figure 5.7: Streaking traces and time delay in photoemission: Yukawa potential. Streaking traces by projecting on PW and SW for positive momenta (color scale in arbitrary units represents the electron density) as a function of the time delay $\tau$ between the XUV and the IR laser pulses. The momentum expectation value $\left\langle p_{\mathrm{R}}\right\rangle$ is depicted inside of the streaking traces in blue circles. In the same plot the vector potential negative $-A_{\mathrm{L}}(\tau)$ is plotted in white lines. c-d) The expectation value $\left\langle p_{\mathrm{R}}^{\prime}\right\rangle=\left\langle p_{\mathrm{R}}\right\rangle-p_{0}$ (blue line with circles) using PW and SW are compared to $-A_{\mathrm{L}}(\tau)$ (red line). Inset graphs show a clear time shift $\Delta t_{\mathrm{S}}$ between the vector potential and the momentum expectation $\left\langle p_{\mathrm{R}}^{\prime}\right\rangle$ when PW or SW are used to compute the streaking spectrogram. The attosecond pulse parameters in these simulations are: frequency $\omega_{\mathrm{X}}=1.2$ a.u., peak intensity $5 \times 10^{12} \mathrm{~W} / \mathrm{cm}^{2}$ and CEP $=0$. This pulse has a gaussian envelope shape with $\mathrm{FWHM}=22.5$ a.u. $(544 \mathrm{as})$. The IR laser pulse frequency is $\omega_{\mathrm{L}}=0.057$ a.u., peak intensity $2.5 \times 10^{12} \mathrm{~W} / \mathrm{cm}^{2}$ and $\mathrm{CEP}=0$. The IR laser pulse envelope is a sine-squared function with two cycles of time-width. 
SW) by the Fourier analysis methods described above, namely $\Delta t_{\mathrm{S} \text {,point }}$ and $\Delta t_{\mathrm{S} \text {,derv }}$. For the short-range Yukawa potential, the method $\Delta t_{\mathrm{S} \text {,point }}$ yields a time delay $\Delta t_{\mathrm{S}}^{(\mathrm{PW})}=$ $\Delta t_{\mathrm{S}}^{(\mathrm{SW})}=-0.48$ a.u., from $\mathrm{PW}$ and SW calculations, respectively. ${ }^{1}$ This streaking time delay is in very good agreement with the averaged Wigner time delay ${ }^{2}\left\langle\Delta t_{\mathrm{W}}\right\rangle=-0.47$ a.u., within the spectra bandwidth of the emitted EWP.
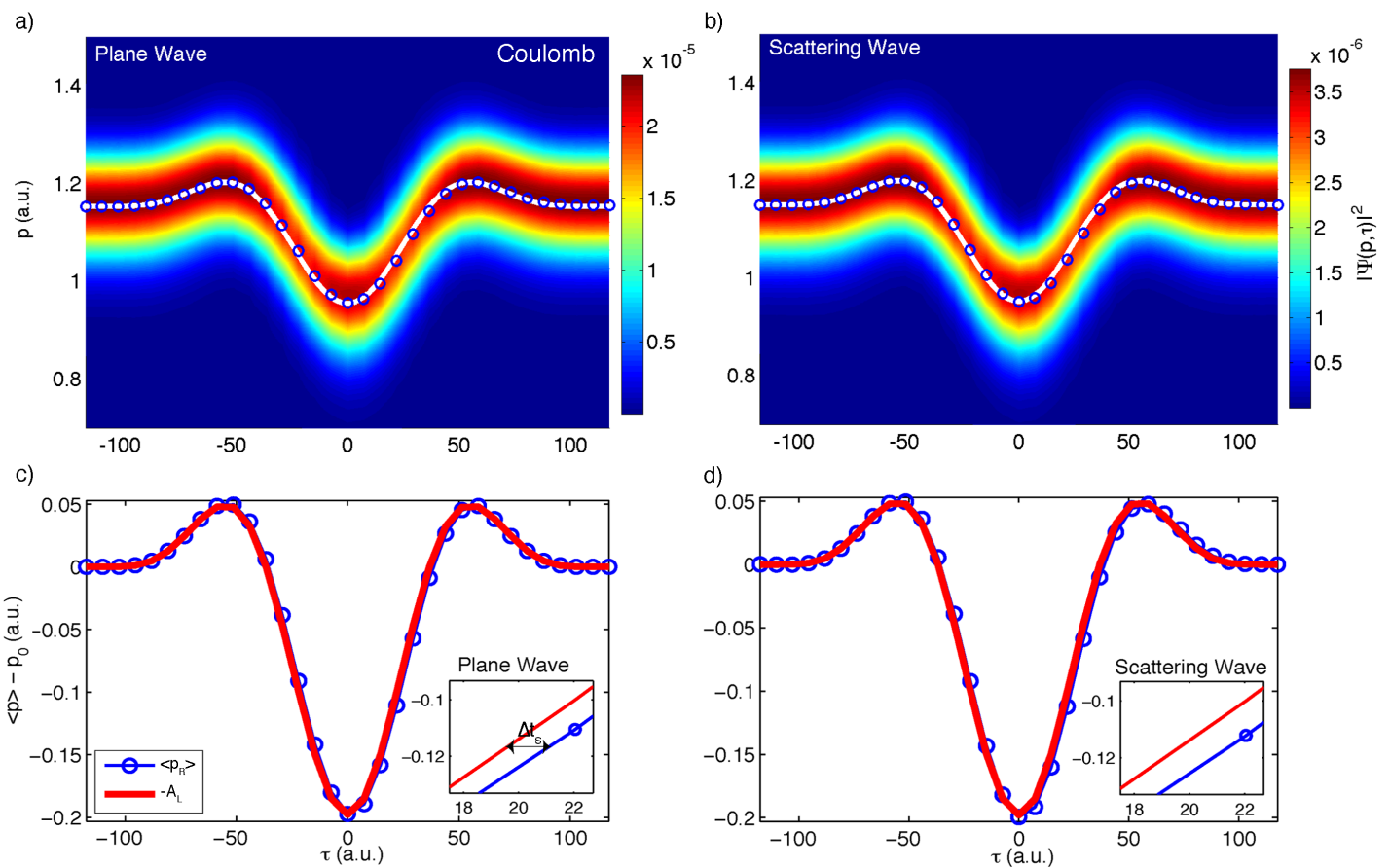

Figure 5.8: Streaking traces and time delay in photoemission: $\mathbf{H}$ Coulomb potential. Exactly the same as Fig. 5.7 but for a Coulomb potential.

However, in the case of a long-range Coulomb potential the streaking time delay $\Delta t_{\mathrm{S}}^{(\mathrm{PW})}=-1.40$ and $\Delta t_{\mathrm{S}}^{(\mathrm{SW})}=-1.50$ a.u., are in disagreement with the exact averaged Wigner time delay $\left\langle\Delta t_{\mathrm{W}}\right\rangle=0.20$ a.u. A similar contradictory result has been obtained in the last Section for a long-range Coulomb potential. Thus, it is a clear that there exists an influence on the attosecond streaking measurement when a long-range potential is present. The key point is to find out from where this disagreement between the streaking time measurement and the Wigner time delay would come from.

To estimate a broader range of streaking time delays, we calculate a set of attosecond

\footnotetext{
${ }^{1}$ The Fourier method $\Delta t_{\mathrm{S}, \text { derv }}$ gave the same result that $\Delta t_{\mathrm{S} \text {, point }}$ (with a relative difference of less than 2). For this reason, we choose the Fourier analysis method $\Delta t_{\mathrm{S} \text {,point }}=\phi\left(\omega_{0}\right) / \omega_{0}$ to extract the streaking time delay.

${ }^{2}$ For instance, see Eq. (A.10) in Section A.4
} 
streaking spectrograms and from them we extract the time delay $\Delta t_{\mathrm{S}}$. We employ attosecond XUV pulses with central frequencies between $1.0-2.2$ a.u., and the same peak intensity. The CEP and FWHM $<25$ a.u., are similar than the ones used in the examples presented in Fig. 5.7. The IR laser pulse parameters are the same.

The streaking time delay as a function of the photoelectron central energy $E_{0}$ is depicted in Fig. 5.9 for the case of short-range and long-range potentials.
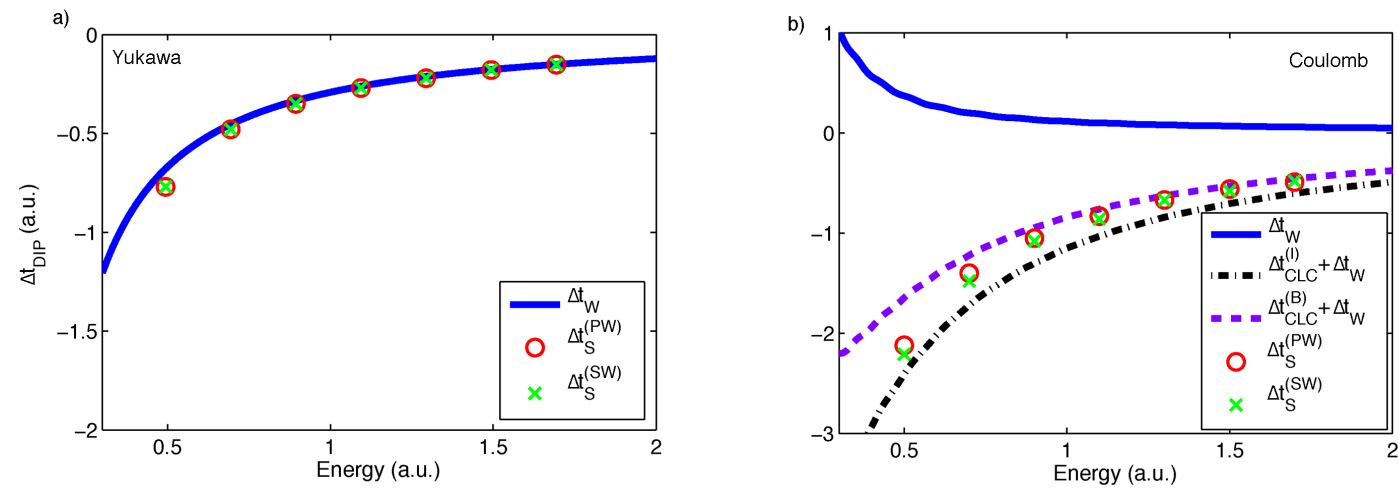

Figure 5.9: Streaking time delay in the short-range and long-range potentials. a) Streaking time delay $\Delta t_{\mathrm{S}}$ by PW (red circles) and $\mathrm{SW}$ (green crosses) as a function of the photoelectron central energy compared to the exact Wigner time delay $\Delta t_{\mathrm{W}}$ (blue line) for the short-range Yukawa potential. b) The same as a) but for the soft-core Coulomb potential. The CLC $\Delta t_{\text {CLC }}^{(\mathrm{I} / \mathrm{B})}$ added up to the Wigner time delay $\Delta t_{\mathrm{W}}$ is shown in a black dashed line (according to Ivanov [74]) and in a violet dashed line (according to Burgdörfer [159]).

In Fig. 5.9a) the streaking time delay extracted from PW, $\Delta t_{\mathrm{S}}^{(\mathrm{PW})}$, and $\mathrm{SW}, \Delta t_{\mathrm{S}}^{(\mathrm{SW})}$, are compared to the exact Wigner time delay, $\Delta t_{\mathrm{W}}$, in the case of a short-range Yukawa potential. We find a perfect agreement between the streaking time delay and the exact one. Both the PW and SW give the same time delay, $\Delta t_{\mathrm{S}}$, as expected for a short-range potential.

For the Coulomb potential the streaking results differ significantly from the Wigner time delay (see Fig. 5.9b)). This disagreement comes from the long-range behaviour of the Coulomb potential and it is also related to the so-called CLC [68, 72, 73, 74, 159]. Ivanov and Smirnova have found an analytical expression for this CLC which is related to the laser frequency and the atomic properties of the system [74]. From the eikonal-Volkov approach and with the WKB approximation they obtain an analytical expression, that reads: 


$$
\Delta t_{\mathrm{CLC}} \approx-\frac{1}{p_{0}^{3}}\left[\ln \left(\frac{2 p_{0}^{2}}{\omega_{\mathrm{L}}}\right)-\gamma_{\text {Euler }}+\frac{\pi \omega_{\mathrm{L}}}{4 p_{0}^{2}}\right]
$$

where $\gamma_{\text {Euler }}=0.5772$ is Euler's constant. We evaluate this CLC time delay contribution $\Delta t_{\mathrm{CLC}}$ and add it up to our 1D calculation of the Wigner time delay $\Delta t_{\mathrm{W}}$. The result is depicted in Fig. 5.9b) and we find a good match between our numerical streaking time delay calculation and the total time delay $\Delta t_{\mathrm{Tot}}=\Delta t_{\mathrm{CLC}}+\Delta t_{\mathrm{W}}$. For the low energy range, we find a deviation of the total time delay with respect to our numerical simulation results. This deviation is likely due to the fact the Eikonal-Volkov approximation is only correct for high photoelectron energies.

Note that our numerical streaking time delay calculated by using PW is in very good agreement with the SW. There is only an small difference for low energies. This is important in the cases where SWs cannot be easily computed. We will use this result as an argument to compute the streaking trace by $\mathrm{PW}$ in $3 \mathrm{D}$ and therefore to extract the Wigner time.
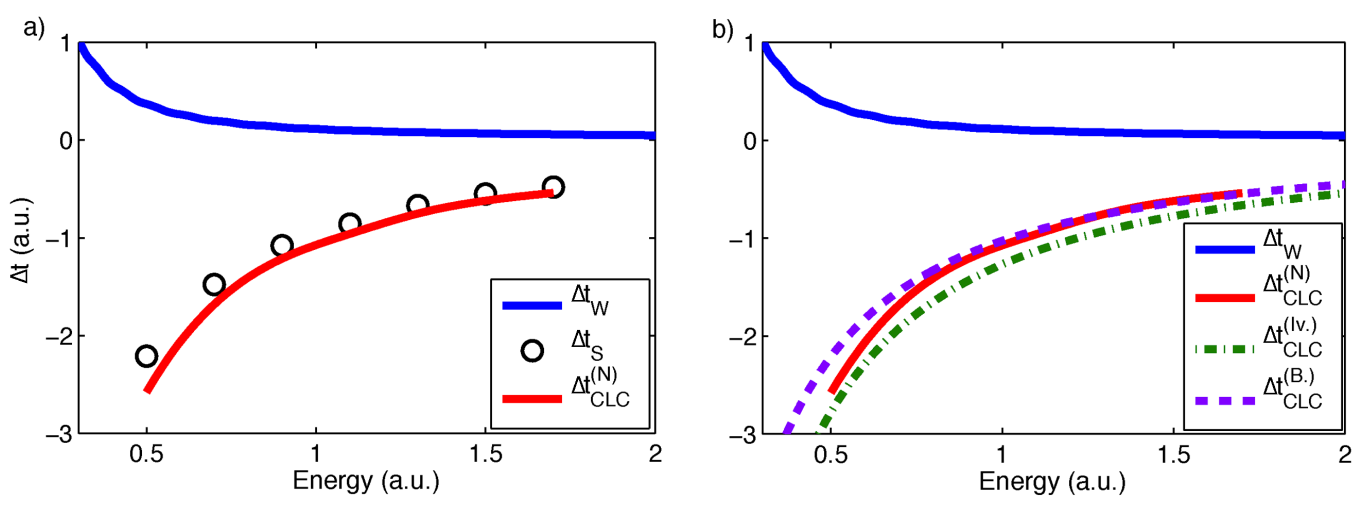

Figure 5.10: Streaking time delay in photoemission for a long-range potential. a) The attosecond streaking time delay (black circles) $\Delta t_{\mathrm{S}}$ as a function of the photoelectron energy compared to the exact Wigner time delay (blue line) $\Delta t_{\mathrm{W}}$, and the numerical CLC time delay $\Delta t_{\mathrm{CLC}}^{(\mathrm{N})}$ in red solid line. b) Detailed analysis of the time delay in photoemission: the Wigner time delay in blue line, and the CLC time delay $\Delta t_{\mathrm{CLC}}$ by the numerical calculation (red line), Ivanov (green dashed line) and Burgdörfer (violet dashed line) approach.

Furthermore, Fig. 5.10a) shows each term of the streaking time delay in photoemission, i.e., the Wigner time delay and the CLC time delay. Fig. 5.10b) depicts detail information about the different approximations developed by Ivanov [74] and 
Burgdörfer in [159]. The difference between the Ivanov and Burgdörfer approaches comes from the derivation procedure used in both cases.

It is demonstrated that the streaking time delay $\Delta t_{\mathrm{S}}$ is not directly related to the Wigner time delay in the photoemission process for long-range potentials and an extra analysis need to be performed. The coupling between the laser and the Coulomb potential distorts the measurement process and it cannot be avoided.

It is probably one of the most important issues in the experiment presented by Schultze et al. in [17]. As Dahlström and coworkers present in [68] the definition of a time delay between two EWPs of different energy is difficult, due to different momenta of the photoelectrons and their logarithmical dependence expressed in Eq. (A.8) (see Section A.3).

Due to the problematic nature to measure the Wigner time delay in atomic system, we will address in the next Section an alternative way to extract it from an orientated asymmetric molecular system. If two EWPs are emitted from the same bound state of an asymmetric system, the asymptotic long-range phase term difference between the electron released on the left and on the right will cancel out [75] but a remaining short-range phase difference will be present.

\subsection{Wigner time delay in a small asymmetric molecule}

The main difficulty to extract the Wigner time delay from an atomic system is related to the fact that during the detection process the coupling between the long-range Coulomb potential and the laser field plays an important role.

In the experiment of Schultze et al. [17], where a measurement of a relative time delay in photoemission from the $2 s$ and $2 p$ of the Ne atom was presented, one would expect that the two launched EWPs to the continuum have different final velocities. This implicates that the contribution of the long-range potential between both channels influences substantially the photoemission process. Then, the CLC needs to be estimated and removed from the measurement in order to extract a "clean" relative Wigner time delay between both orbitals.

An alternative process to avoid this CLC is to compare the emission of two EWPs propagating with the same final energy. Here we will show that by choosing an asymmetric orientated molecule as CO, this CLC could be removed from the detection process by measuring the asymmetric left-right time delay.

We introduce a 1D model within the SAE approach which is similar to those used in the literature for CO [160]. In addition we fix the positions of the nuclei by considering 
their dynamics is much slower than the electron movement. This model allows us to calculate the exact scattering continuum waves and the complex dipole transition matrix element, from which the Wigner time delay can be computed directly.

\subsubsection{Description of the asymmetric system}

In order to mimic a oriented $\mathrm{CO}$ molecule in $1 \mathrm{D}$ along the laser polarization axis we choose a soft-core Coulomb potential to model the two nuclei centers:

$$
V_{\mathrm{CO}}(z)=-\frac{Z_{1}}{\sqrt{a_{1}+\left(z-R_{1}\right)^{2}}}-\frac{Z_{2}}{\sqrt{a_{2}+\left(z-R_{2}\right)^{2}}},
$$

where $Z_{1}$ and $Z_{2}$ are the charge of each core, $a_{1}$ and $a_{2}$, the soft-core parameters and $R_{1}$ and $R_{2}$, the position of the nuclei. We use for the orientated molecule charge parameters $Z_{1}=0.67, Z_{2}=1-Z_{1}=0.33$, soft-cores $a_{1}=1.60, a_{2}=1.33$ and core positions $R_{1}=-0.6$ and $R_{2}=1.65$ a.u. These parameters are chosen such that the ground-state energy matches the ionization potential energy $I_{p}=0.5$ a.u., and the internuclear distance is $R=2.25$ a.u., which are close to the values prescribed by an actual CO molecule [161]. The ground-state of the system is calculated by imaginary time propagation with $\Delta t=-0.01 i$ a.u.

The asymmetric positions of the cores $R_{1}$ and $R_{2}$ are chosen to place the maximum of the electron density at zero position as it is depicted in Fig. 5.11. This is to avoid any artificial time delay introduced by the initial electron position with respect to the maximum of the electron position density. The grid parameters are the same that those used in Section 5.2.1.

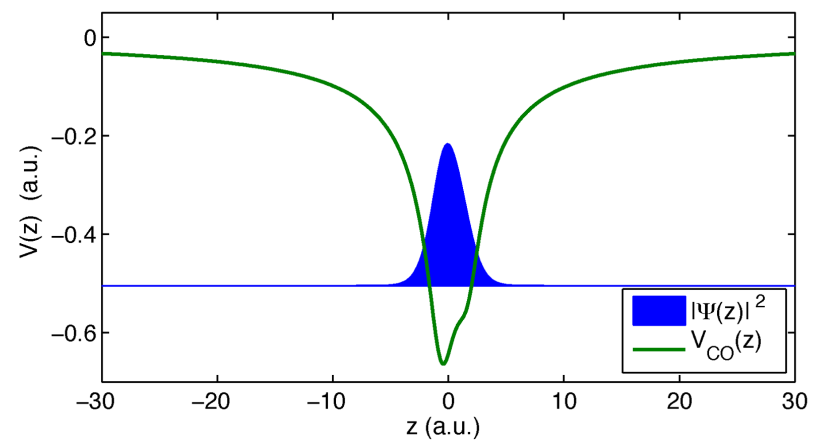

Figure 5.11: 1D Asymmetric model for the carbon monoxide (CO). The potential $V_{\mathrm{CO}}(z)$ is depicted in green dark line and the electron density of the ground state (blue are) associated to this potential. 
Fig. 5.11 shows the structure of the potential and the ground state which model our one dimensional approach of the $\mathrm{CO}$ molecule. The potential $V_{\mathrm{CO}}(z)$ shows a small asymmetry as well as the typical long-range Coulomb behaviour. In order to simplify our analysis we have chosen a ground state similar to the ground state of the hydrogen atom.

While this state apparently looks symmetric it is not the case and its structural asymmetric information will be reflected in the bound-free dipole transition matrix element and specially in the dipole phase.

In the next Section we will compute the dipole, starting form this bound state, the dipole phase and the Wigner time delay will then be calculated. The Winger time delay for an electron with positive and negative momentum will be defined and in addition the so-called left-right asymmetry.

\subsubsection{Dipole structure and stereo Wigner time}

To compute the Wigner time delay for the molecular system we follow exactly the same steps that in Section 5.2.1, i.e., to compute the bound-free dipole transition matrix element by PW and SW. The Wigner time delay is calculated according to the definition given by Eq. (5.1).

Fig. 5.12 shows the amplitude $|d(k)|$ and phase $\phi(k)$ of the complex dipole transition matrix element as a function of the momentum $k$ by PW and SW.
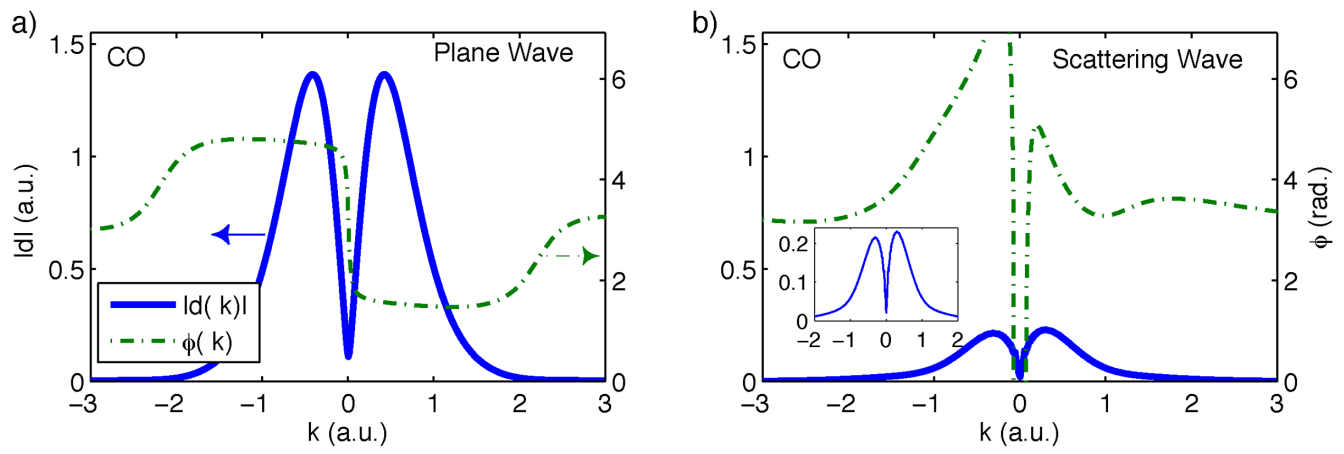

Figure 5.12: Asymmetric CO molecular dipole transition matrix element. a) The amplitude (blue line) and phase (dots-dashed green line) of the bound-free dipole transition matrix element by considering the continuum state as $\mathrm{PW}, \Psi_{k}(z)=\frac{1}{\sqrt{2 \pi}} e^{i k z}$. b) The same as a) but with $\mathrm{SW}, \Psi_{k}(z)$.

As we expect the PW yields different results than SW for the complex dipole. The 
dipole amplitude in case of PW projection is totally symmetric while in the case of SW it is slightly asymmetric. The dipole phase also differs strongly whether PW or SW is used to compute it. While the asymmetry occurs in the dipole transition matrix element amplitude and phase, the asymmetry in the amplitude is small and probably very difficult to measure. In contrast, the asymmetry in the dipole phase is much larger and therefore sensitive to the details of the asymmetric potential. Thus, it configures a more powerful observable to measure the characteristics of the asymmetric molecule $(\mathrm{AM})$.

We introduce a systematic definition for the asymmetric time delay. We define the left Wigner time delay as the derivative of the dipole phase with respect to the energy for electrons with negative momentum $\Delta t_{\mathrm{W}}^{(\mathrm{L})}=\left.\frac{1}{k} \frac{\partial \phi(k)}{\partial k}\right|_{k<0}$. The right Wigner time delay, on the other hand, is defined as $\Delta t_{\mathrm{W}}^{(\mathrm{R})}=\left.\frac{1}{k} \frac{\partial \phi(k)}{\partial k}\right|_{k>0}$ for electrons with positive momentum. Then, the asymmetric time delay or the Stereo Wigner Time Delay is defined as the difference between these two quantities:

$$
\Delta t_{\mathrm{W}}^{(\mathrm{LR})}=\Delta t_{\mathrm{W}}^{(\mathrm{L})}-\Delta t_{\mathrm{W}}^{(\mathrm{R})}
$$

Fig. 5.13 shows the calculations for the left-right Wigner time delay and the SWTD from the dipole phases computed by the PW and SW. The left and right Wigner time delays are different if PWs or SWs are used to compute them. However, for both methods, a clear minimum is obtained in the stereo Wigner time delay. It is clear that different information are obtained for the stereo Wigner time if PWs or SWs are used.
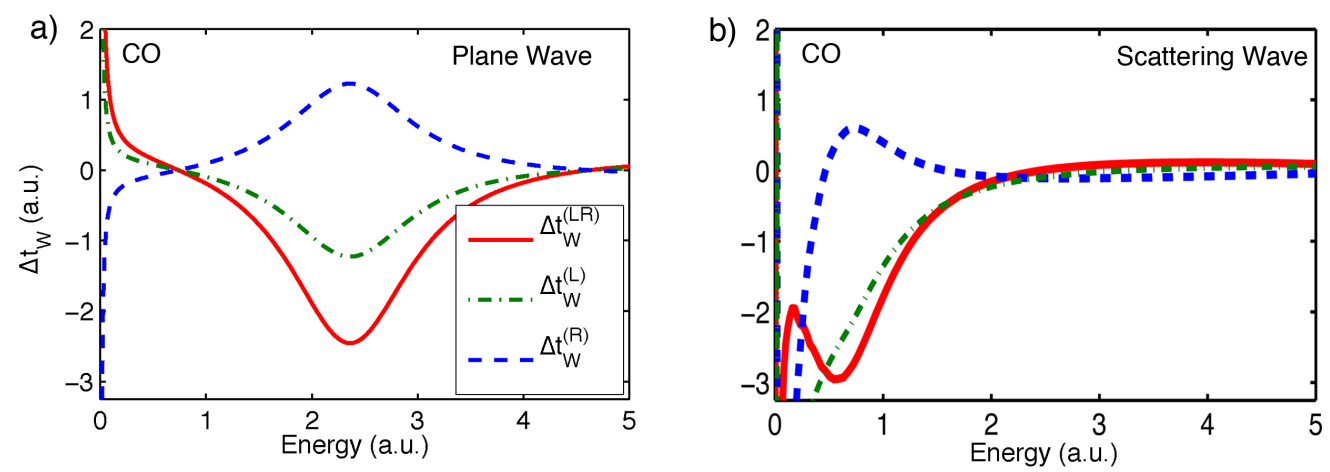

Figure 5.13: Stereo Wigner time delay. a) The left, right and stereo Wigner time delays by PW calculations are depicted in dots-dashed green, dashed blue and red lines, respectively. b) The same as a) but for SW calculations.

The SWTD presented in Fig. 5.13 indicates the asymmetric characteristics of the po- 
tential model for the $\mathrm{CO}$ molecule which physically means that an emitted EWP to the left side leaves from the molecule before than an emitted to the right. This is an important fact because this asymmetry indicates that the orientation of the molecule plays a special role in the detection process. Thus, it gives information about the nuclei locations of the molecule.

\subsubsection{Photoionization form an asymmetric molecule}

In this Section we will describe how to extract the stereo Wigner time delay information by using a semiclassical analysis to the emitted, to the left or to the right, EWPs launched from the interaction of a SAP with the oriented CO molecule.

Similarly to Section A.4, we will use the extrapolation method to extract the absolute time delay in photoemission on both sides and from it to compute the stereo Winger time delay. In addition, we also recover the SWTD by the time of flight [77] and compare it to the results obtained from the extrapolation method.

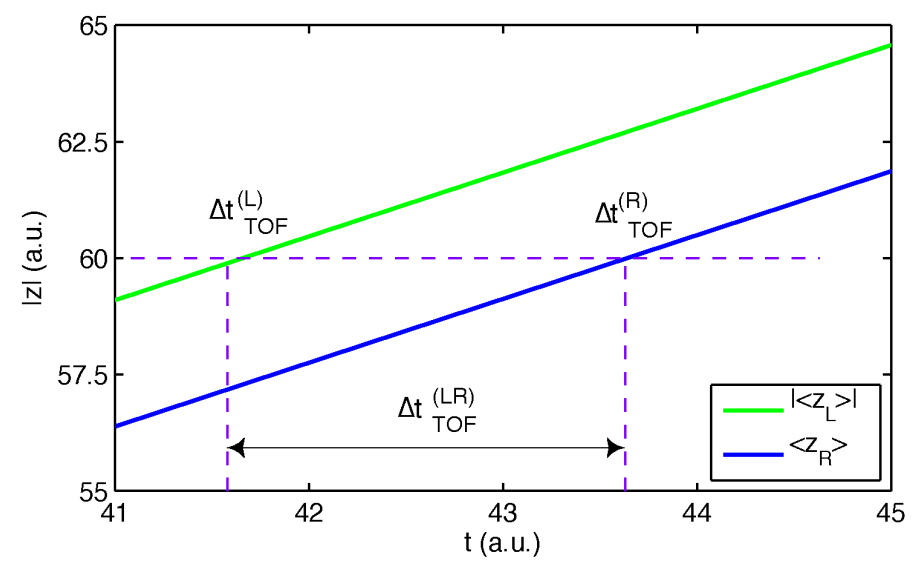

Figure 5.14: Scheme for the stereo TOF. Schematic representation of the time of flight for EWPs emitted on left $\Delta t_{\mathrm{TOF}}^{(\mathrm{L})}$, right $\Delta t_{\mathrm{TOF}}^{(\mathrm{R})}$, and the stereo TOF $\Delta t_{\mathrm{TOF}}^{(\mathrm{LR})}$. The expectation values on left, $\left\langle z_{\mathrm{L}}\right\rangle$, and right, $\left\langle z_{\mathrm{R}}\right\rangle$, as a function of time are depicted in green and blue lines, respectively.

We define the TOF method as the time $\Delta t_{\mathrm{TOF}}=t_{d}-t_{0}$ that an EWP spends in the continuum from an initial time $t_{0}$ until the arrival time $t_{d}$, at a certain position $z_{d}$. This simple method allows us to extract the asymmetry in the TOF of EWPs released to the left and to the right.

Fig. 5.14 shows an schematic representation of the stereo TOF method. This consists to follow the semiclassical electron trajectories on either side, $\left\langle z_{\mathrm{L} / \mathrm{R}}\right\rangle$, as a function of 
time. Then, the detection of symmetric positions on the left and right, $\left|\left\langle z_{\mathrm{L}}\right\rangle\right|=\left\langle z_{\mathrm{R}}\right\rangle$, is fixed to extract the corresponding left $\Delta t_{\mathrm{TOF}}^{(\mathrm{L})}$ and right $\Delta t_{\mathrm{TOF}}^{(\mathrm{R})}$ arrival times. The difference $\Delta t_{\mathrm{TOF}}^{(\mathrm{LR})}=\Delta t_{\mathrm{TOF}}^{(\mathrm{L})}-\Delta t_{\mathrm{TOF}}^{(\mathrm{R})}$ defines the stereo TOF of the emitted EWPs on both sides.

\section{Implementation of the method}

We numerically solve the TDSE to simulate the EWP emissions on either side from the ground states of an $\mathrm{H}$ atom and a $\mathrm{CO}$ molecule by a SAP. The left-right $\left\langle z_{\mathrm{L} / \mathrm{R}}\right\rangle$ position expectation values for the EWPs released on both sides, are computed as a function of time and depicted in Fig. 5.15 for an $\mathrm{H}$ atom and a $\mathrm{CO}$ molecule ${ }^{1}$.

In case of $\mathrm{H}$, the straight line trajectories, $z_{\mathrm{M}, \mathrm{L} / \mathrm{R}}(t)$, are calculated by evaluating the instantaneous velocities on the left-right $v_{\mathrm{L} / \mathrm{R}}\left(t_{d}\right)=\mp 1.359$ a.u., at the time $t_{d}=447.67$ a.u., and at the corresponding positions $\left\langle z_{\mathrm{L} / \mathrm{R}}\right\rangle=\mp 611.2$ a.u.

In the inset graph of Fig. 5.15a) we show that the black dashed vertical line coincides exactly with the intersection of the back extrapolation left $z_{\mathrm{M}, \mathrm{L}}(t)$ and right $z_{\mathrm{M}, \mathrm{R}}(t)$ trajectories, to the zero position $z=0$. The relative time delays between the maximum of the XUV envelope (see, red vertical dashed line in inset graph of Fig. 5.15a)) and the time intersection points at the zero position (see, black vertical dashed line in inset graph of Fig. 5.15a)) yield $\Delta t_{\mathrm{DIP}}^{(\mathrm{L})}=\Delta t_{\mathrm{DIP}}^{(\mathrm{R})}=-2.1$ a.u. Then, it is clear that the stereo time delay in photoemission is zero in case of $\mathrm{H}$.

On the other hand, in case of a $\mathrm{CO}$ molecule, the matched straight line trajectories are calculated by evaluating the instantaneous velocity on the left $v_{\mathrm{L} / \mathrm{R}}=\mp 1.358$ at the time-positions $(447.67,-612.1)$ and at $(447.67,609.5)$ a.u., respectively. The back extrapolation method shown in the inset graph of Fig. 5.15b) yields different time delays in photoemission for the emitted EWPs on either side $\Delta t_{\mathrm{DIP}}^{(\mathrm{L} / \mathrm{R})}$.

Table 5.1 shows a comparison of these time delay values, $\Delta t_{\mathrm{DIP}}^{(\mathrm{L} / \mathrm{R})}$ and the stereo time delay, $\Delta t_{\mathrm{DIP}}^{(\mathrm{LR})}$, to the exact Wigner time delays $\left\langle\Delta t_{\mathrm{W}}^{(\mathrm{L} / \mathrm{R})}\right\rangle$ and $\left\langle\Delta t_{\mathrm{W}}^{(\mathrm{LR})}\right\rangle$, respectively. The left and right averaged Wigner time delays are computed by the definition given in Eq. (A.10) of the Section A.4. In order to perform these calculations, we have taken into account the photoelectron energy distributions for the released EWPs on either side. The stereo time delay in photoemission $\Delta t_{\mathrm{TOF}}^{(\mathrm{LR})}$ by the TOF method is also shown in the Table 5.1.

We find that while the left-right time delays in photoemission are in disagreement with the exact Wigner time delays, the stereo time delays by the extrapolation method $\Delta t_{\mathrm{DIP}}^{(\mathrm{LR})}$ and TOF method $\Delta t_{\mathrm{TOF}}^{(\mathrm{LR})}$, are in very good agreement with the exact averaged

\footnotetext{
${ }^{1}$ For more detail, see Sections A.1 and A.2 of Appendix A
} 
a)

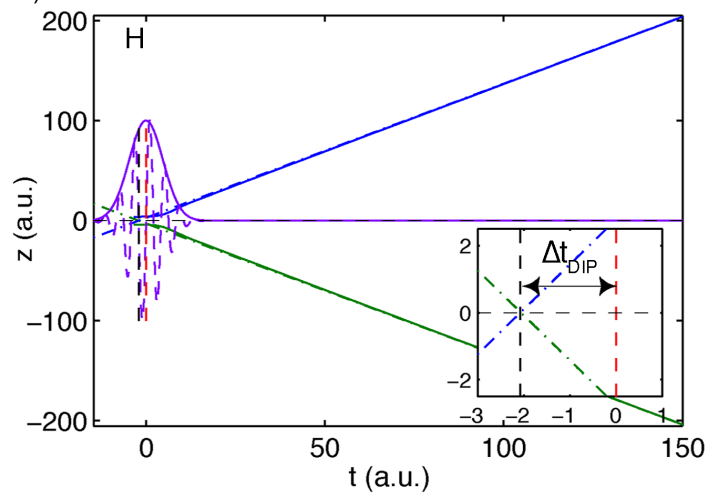

b)

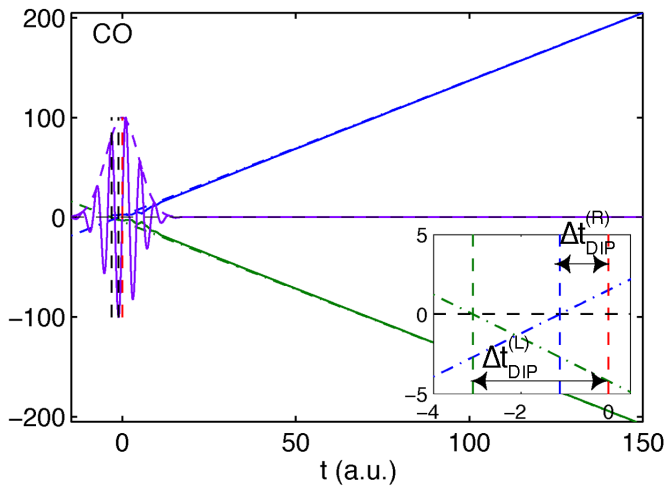

Figure 5.15: Left and right semiclassical trajectories. a-b) The position expectation values for EWPs emitted (from the $\mathrm{H}$ atom and $\mathrm{CO}$ molecule) to the left, $\left\langle z_{\mathrm{L}}\right\rangle$, and right, $\left\langle z_{\mathrm{R}}\right\rangle$, as a function of time $t$ are shown in green and blue line, respectively. In dots-dashed green and dots-dashed blue lines the match trajectories to the left $z_{\mathrm{M}, \mathrm{L}}(t)$ and to the right $z_{\mathrm{M}, \mathrm{R}}(t)$, respectively. The inset graphs depict a zoom around the origin of coordinates and the extrapolation of trajectories $z_{\mathrm{M}, \mathrm{L} / \mathrm{R}}$. The time delay in photoemission $\Delta t_{\mathrm{DIP}}$ by the back extrapolation method is indicated by the time between the red and the black dashed vertical lines in case of $\mathrm{H}$ (inset graph in a)). The red vertical dashed line defines the time at the maximum of the XUV envelope. For CO, $\Delta t_{\mathrm{DIP}}^{(\mathrm{L})}$ denotes the time delay in photoemission for the emitted EWPs to the left and $\Delta t_{\mathrm{DIP}}^{(\mathrm{R})}$ right (inset graph in b)). It is clear that in case of CO there is an asymmetry $\Delta t_{\mathrm{DIP}}^{(\mathrm{LR})}=\Delta t_{\mathrm{DIP}}^{(\mathrm{L})}-\Delta t_{\mathrm{DIP}}^{(\mathrm{R})}$. The $\mathrm{SAP}$ parameters used in these simulations are: peak intensity $I_{\mathrm{X}}=10^{12} \mathrm{~W} / \mathrm{cm}^{2}$, central frequency $\omega_{\mathrm{X}}=1.5$ a.u.. In addition the pulse has a $\mathrm{FWHM}=9.5$ a.u. with a gaussian envelope and a $\mathrm{CEP}=0$. 


\begin{tabular}{|c|c|c|c|c|c|c|}
\hline$\left\langle\Delta t_{\mathrm{W}}^{(\mathrm{L})}\right\rangle$ & $\Delta t_{\mathrm{DIP}}^{(\mathrm{L})}$ & $\left\langle\Delta t_{\mathrm{W}}^{(\mathrm{R})}\right\rangle$ & $\Delta t_{\mathrm{DIP}}^{(\mathrm{R})}$ & $\left\langle\Delta t_{\mathrm{W}}^{(\mathrm{LR})}\right\rangle$ & $\Delta t_{\mathrm{DIP}}^{(\mathrm{LR})}$ & $\Delta t_{\mathrm{TOF}}^{(\mathrm{LR})}$ \\
\hline-1.60 & -3.10 & 0.47 & -1.1 & -2.07 & -2.00 & -1.98 \\
\hline \hline
\end{tabular}

Table 5.1: Delay in photoemission for the asymmetry molecule. Here, atomic units are used.

of the stereo Wigner time delay $\left\langle\Delta t_{\mathrm{W}}^{(\mathrm{LR})}\right\rangle$. This indicates us a clear asymmetry in the photoemission time delay for the $\mathrm{CO}$ molecule.

We scan the absolute time delays on the left, $\Delta t_{\mathrm{DIP}}^{(\mathrm{L})}$, and right, $\Delta t_{\mathrm{DIP}}^{(\mathrm{R})}$, by the extrapolation method for different position expectation values on both sides $^{1}$. Then, we calculate $\Delta t_{\mathrm{DIP}}^{(\mathrm{LR})}$ as a function of the symmetric positions $\left|z_{\mathrm{L} / \mathrm{R}}\right|$. This method allows us to remove asymptotically the logarithmic delay term related to the long-range Coulomb behaviour.

In addition, we estimate the total time delays on the left and on the right defining:

$$
\Delta t_{\mathrm{Tot}}^{(\mathrm{L} / \mathrm{R})}=\Delta t_{\mathrm{L} \cdot-\mathrm{R} .}^{(\mathrm{L} / \mathrm{R})}+\Delta t_{\mathrm{W}}^{(\mathrm{L} / \mathrm{R})}+\frac{z_{0}}{k_{0}} .
$$

Then, we evaluate the long-range time delay contributions on either side using:

$$
\Delta t_{\mathrm{L} \cdot-\mathrm{R} .}^{(\mathrm{R})}=\frac{Z_{\mathrm{Tot}}}{k_{0}^{3}}\left(1-\ln \left|2 k_{0} z_{\mathrm{L} / \mathrm{R}}\right|\right),
$$

and by taking symmetric positions $\left|z_{\mathrm{L} / \mathrm{R}}\right|$, momentum $k_{0}=\left|\left\langle k_{\mathrm{L}}\right\rangle\right|=\left\langle k_{\mathrm{R}}\right\rangle$ and the total charge $Z_{\mathrm{Tot}}=Z_{1}+Z_{2}$ of our CO model. The Wigner time delays $\Delta t_{\mathrm{W}}^{(\mathrm{L} / \mathrm{R})}$ are evaluated on both sides at the momentum $\mp k_{0}$. The extra time delay contribution $z_{0} / k_{0}$ is computed by choosing arbitrarily the value $z_{0}$ such that the time delays $\Delta t_{\mathrm{L} .-\mathrm{R}}^{(\mathrm{L} / \mathrm{R})}+$ $\Delta t_{\mathrm{W}}^{(\mathrm{L} / \mathrm{R})}$ matches the $\Delta t_{\mathrm{DIP}}^{(\mathrm{L} / \mathrm{R})}$ ones. This position $\left|z_{0}\right|$ is the same for both sides.

The results for the estimated total left-right time delays and for the numerical stereo photoemission time delays are depicted in Fig. 5.16. The left and right time delays in photoemission show a clear logarithmic behaviour (see Fig. 5.16a)) as it can be expected. We compare the estimated total time delays $\Delta t_{\mathrm{Tot}}^{(\mathrm{L} / \mathrm{R})}$ to the photoemission ones on either side $\Delta t_{\mathrm{DIP}}^{(\mathrm{L} / \mathrm{R})}$ in Fig. 5.16a). We find a very good agreement between the photoemission tiem delays $\Delta t_{\mathrm{DIP}}^{(\mathrm{L} / \mathrm{R})}$ and the estimated total time delay $\Delta t_{\mathrm{Tot}}^{(\mathrm{L} / \mathrm{R})}$.

We note that due to the long-range Coulomb tail of the CO potential on either side, the delays $\Delta t_{\mathrm{DIP}}^{(\mathrm{L} / \mathrm{R})}$ do not converge to the well defined time delays as it is expected. However, as we show in Fig. 5.16b) by taking the difference left-right $\Delta t_{\mathrm{DIP}}^{(\mathrm{LR})}$, this logarithmic behavior disappears and a very good convergence to a well defined stereo

\footnotetext{
${ }^{1}$ To simplify the notation, we denote $\left\langle z_{\mathrm{L} / \mathrm{R}}\right\rangle=z_{\mathrm{L} / \mathrm{R}}$.
} 

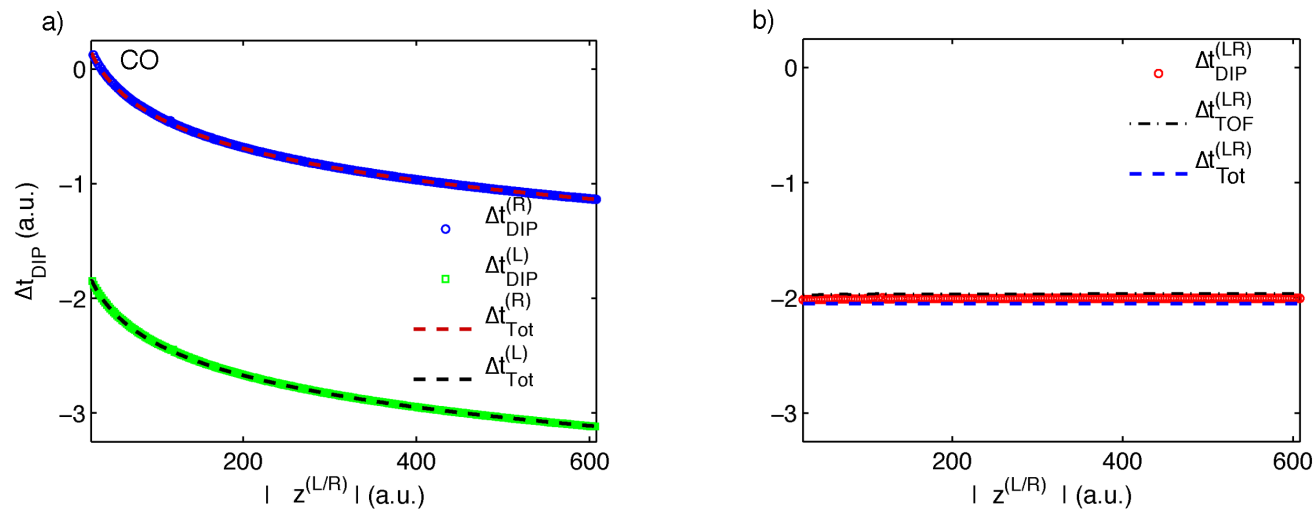

Figure 5.16: Extrapolation and TOF methods to extract stereo Wigner time delay. By the extrapolation method, the absolute time delays for the emitted EWPs to the left, $\Delta t_{\mathrm{DIP}}^{(\mathrm{L})}$, (green circles) and to the right, $\Delta t_{\mathrm{DIP}}^{(\mathrm{R})}$, (blue circles) as a function of the symmetric positions $\left|\left\langle z_{\mathrm{L}}\right\rangle\right|=\left\langle z_{\mathrm{R}}\right\rangle$ are shown in a). The estimated total exact time delay in photoemission for either side $\Delta t_{\text {Tot }}^{(\mathrm{L} / \mathrm{R})}$ are depicted in black dashed and red dashed lines, respectively. b) The stereo time delay in photoemission, $\Delta t_{\mathrm{DIP}}^{(\mathrm{LR})}$, by the extrapolation (red circles) and the TOF (dots-dashed black line) methods, is compared to the exact stereo total time delay or stereo Wigner time delay (blue dashed line).

time delay $\Delta t_{\mathrm{DIP}}^{(\mathrm{LR})}$ is obtained. Note that this stereo time delay is in very good agreement with the asymmetric total time delay $\Delta t_{\text {Tot }}^{(\mathrm{LR})}$ which is equal to the stereo Wigner time delay $\Delta t_{\mathrm{Tot}}^{(\mathrm{LR})}=\Delta t_{\mathrm{W}}^{(\mathrm{LR})}$.

The stereo time delay in photoemission $\Delta t_{\mathrm{TOF}}^{(\mathrm{LR})}$ computed by using the stereo TOF method yields the same results as the stereo photoemission time delay $\Delta t_{\mathrm{DIP}}^{(\mathrm{LR})}$ calculated by employing the extrapolation method within the whole position range of Fig. 5.16b). The difference between the TOF and the extrapolation methods is hardly visible, about 0.05 a.u.

We can conclude that both methods are well suited to estimate numerically the stereo time delay in the photoemission process. For positions close to the nuclei cores, i. e., $z_{(\mathrm{L} / \mathrm{R})}=28$ a.u., or less, the stereo time delay $\Delta t_{\mathrm{DIP}}^{(\mathrm{LR})}$ changes slightly. This small variation is due to the influence of the potential asymmetry around the cores.

In order to prove that the stereo time delay in photoemission can be extracted within a broad range of photoelectron energy, we calculate the EWPs for a set of different XUV attosecond pulses with central frequencies between $\omega_{\mathrm{X}}=1.5-3.0$ a.u., and $\mathrm{FWHM}=9.5,7.2,5.7$ and 4.8 a.u., respectively. The peak intensity is the same and has the value of $I_{\mathrm{X}}=10^{12} \mathrm{w} / \mathrm{cm}^{2}$ and the CEP is set to zero for all the attosecond 
pulses used.

In Fig. 5.17, we show the results for the stereo time delay in photoemission $\Delta t_{\mathrm{TOF}}^{(\mathrm{LR})}$ compared to the exact stereo Wigner time delay $\Delta t_{\mathrm{W}}^{(\mathrm{LR})}$ which is computed via the partial derivative of the dipole phase.

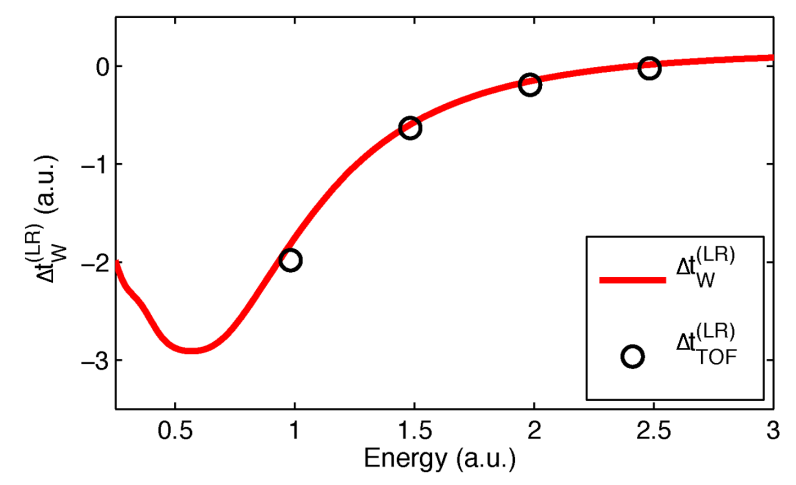

Figure 5.17: CO stereo time delay in photoemission. The stereo Wigner time delay $\Delta t_{\mathrm{W}}^{(\mathrm{LR})}$ (in red line) is compared to the stereo time delay in photoemission $\Delta t_{\mathrm{TOF}}^{(\mathrm{LR})}$ (black circles) by the TOF method as a function of the photoelectron energy.

We find a very good agreement between the stereo time delay in photoemission extracted via the TOF method with the exact stereo Wigner time delay.

The TDSE numerical results presented in this Section show that the left and right absolute photoemission time delays for the orientated CO molecule depend strongly on the detection positions $z_{\mathrm{L} / \mathrm{R}}$ and have asymptotically the same long-range Coulomb behaviour as in the case of an $\mathrm{H}$ Coulomb potential. In contrast, from the stereo photoemission time delay for the released EWPs on either side, the long-range Coulomb term is cancelled out. Then, the stereo photoemission time delay by utilizing the stereo TOF or extrapolation methods, is well defined and perfectly linked to the stereo Wigner time delay.

It is important to note that this stereo photoemission time delay can be a powerful method to remove the so problematic long-range CLC in the measurement process of the fundamental Wigner time delay and specifically the stereo time delay.

As it is impossible to track on time the expectation position value of an EWP ionized by an SAP, the method presented in this Section is not suitable as a measurement scheme in the laboratory. In the next Section we will address how this stereo Wigner time delay can be measured by employing attosecond science tools. 


\subsubsection{Stereo streaking technique in an asymmetric molecule}

The attosecond streak camera has demonstrated to be a powerful tool to study the ultrafast dynamics in atoms and molecules. In this Section we propose a pump-probe attosecond streaking technique to extract the stereo Wigner time delay in the photoemission process similar to the Section 5.2.2. The main difference is that two streaking traces, one on the left and another one on the right, will be needed in order to mimic the measurement of the stereo time delay.

The basic assumption of the streaking technique is that the IR field does not modify neither the initial state nor the final continuum state except for a momentum shift $A_{\mathrm{L}}(\tau)$. However, while the IR laser field effects can be neglected in the initial state, in the continuum the coupling between the laser field and the Coulomb potential produces a time delay which needs to be removed from the measured one [72, 74, 159]. Although this may be possible, the dependence of the CLC on the laser parameters makes this task complicated.

The SWTD $\Delta t_{\mathrm{W}}^{(\mathrm{LR})}$ removes the CLC from the measurements because it is identical on the left and on the right [74]. The SWTD avoids the need to estimate this contribution for each ionization channel, but the SWTD can only be applied to asymmetric systems.

We compute the streaking traces using the TDSE which allows us to simulate the interaction of a SAP in the presence of a weak IR laser field and record the final electron momentum density at the end of both pulses. We perform this task for each time delay $\tau$ between the XUV attosecond pulse and the IR laser field. The attosecond pulse parameters are: peak intensity $I_{\mathrm{X}}=5 \times 10^{12} \mathrm{~W} / \mathrm{cm}^{2}$, central frequency $\omega_{\mathrm{X}}=1.2$ a.u., a gaussian envelope with $\mathrm{FWHM}=20$ a.u., and $\mathrm{CEP}=0 \mathrm{rad}$. The IR laser field has a typical streaking peak intensity of $I_{0}=2.5 \times 10^{12} \mathrm{~W} / \mathrm{cm}^{2}$, central frequency $\omega_{0}=0.057$ a.u., a sine-squared envelope with two total cycles and a CEP $=0$ rad. The grid parameters are the same as in previous Sections.

Fig. 5.18a-b) show the streaking traces for $\mathrm{H}$ and $\mathrm{CO}$, respectively. The electron momentum distributions are computed by projecting the final wave function on SW for both the atomic and molecular cases. To extract the stereo time delay in photoemission from the streaking traces, we compute the momentum expectation values $\left\langle p_{\mathrm{L}}\right\rangle$ and $\left\langle p_{\mathrm{R}}\right\rangle$ as a function of the time delay $\tau$. By the Fourier analysis method $\left(\Delta t_{\mathrm{S}}^{(\mathrm{L} / \mathrm{R})}=\frac{\phi^{(\mathrm{L} / \mathrm{R})}\left(\omega_{0}\right)}{\omega_{0}}\right)$ described in Section 5.2.2 for negative and positive momentum expectation values, the streaking time delays $\Delta t_{\mathrm{S}}^{(\mathrm{L} / \mathrm{R})}$ are extracted. Then, the stereo streaking time delay is recovered by:

$$
\Delta t_{\mathrm{S}}^{(\mathrm{LR})}=\Delta t_{\mathrm{S}}^{(\mathrm{L})}-\Delta t_{\mathrm{S}}^{(\mathrm{R})} .
$$



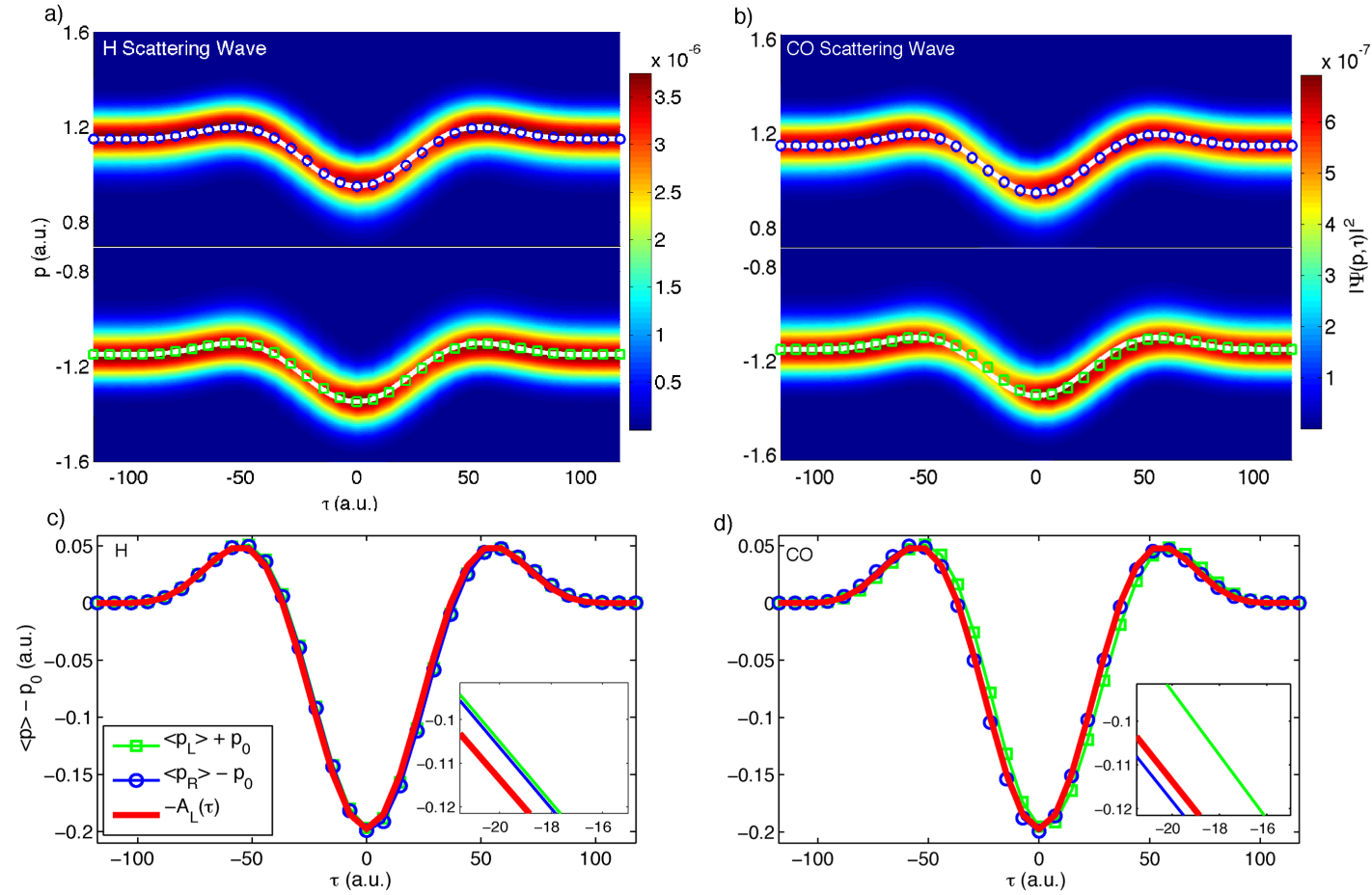

Figure 5.18: Comparison of the streaking traces from $\mathbf{H}$ and $\mathbf{C O}$ systems. a-b) The left-right final momentum distributions, by projecting on SW, as a function of the time delay $\tau$ between the IR and XUV and for $\mathrm{H}$ and CO, respectively. The momentum expectation values for electrons with negative, $\left\langle p_{\mathrm{L}}\right\rangle$, and positive, $\left\langle p_{\mathrm{R}}\right\rangle$, momentum in blue circles (right) and green circles (left). In white line, the vector potential $-A_{\mathrm{L}}(\tau)$. These expectation values and the vector potential $-A_{\mathrm{L}}(\tau)$ for both system are depicted in c) and d). The inset graphs in c) and d) show the streaking time $\Delta t_{\mathrm{S}}^{(\mathrm{L} / \mathrm{R})}$ and the stereo time delay $\Delta t_{\mathrm{S}}^{(\mathrm{LR})}$, between the IR vector potential and the expectation momentum values for both systems.

The shifted momentum expectation values $\left\langle p_{\mathrm{L}}^{\prime}\right\rangle=\left\langle p_{\mathrm{L}}\right\rangle+p_{0}$ and $\left\langle p_{\mathrm{R}}^{\prime}\right\rangle=\left\langle p_{\mathrm{R}}\right\rangle-p_{0}$ are depicted in Fig. $5.18 \mathrm{c}-\mathrm{d}$ ) for the case of $\mathrm{H}$ and $\mathrm{CO}$ systems. As the relative time delays $\Delta t_{\mathrm{S}}^{(\mathrm{L} / \mathrm{R})} \approx-1.5$ between the vector potential $-A_{\mathrm{L}}(\tau)$ and $\left\langle p_{\mathrm{L} / \mathrm{R}}\right\rangle$ are the same, the stereo streaking time delay $\Delta t_{\mathrm{S}}^{(\mathrm{LR})}$ yields a value of zero for the $\mathrm{H}$ atomic case (see inset graph in Fig. 5.18c)).

In contrast, our Fourier analysis gives the streaking times on the left and on the right about $\Delta t_{\mathrm{S}}^{(\mathrm{L})} \approx-2.8$ and $\Delta t_{\mathrm{S}}^{(\mathrm{R})} \approx 0.2$ a.u., for the $\mathrm{CO}$ molecule. Then, the stereo streaking time delay is not zero and has a value of around $\Delta t_{\mathrm{S}}^{(\mathrm{LR})} \approx-3.0$ a.u., as it shows the inset graph in Fig. 5.18d). We note that this stereo streaking time delay is in very good agreement with the exact average of the $\operatorname{SWTD}\left\langle\Delta t_{\mathrm{W}}^{(\mathrm{LR})}\right\rangle=-2.9$ a.u. There is only a small difference which comes from the error of the Fourier analysis, which in 
this case is around 0.1 a.u.
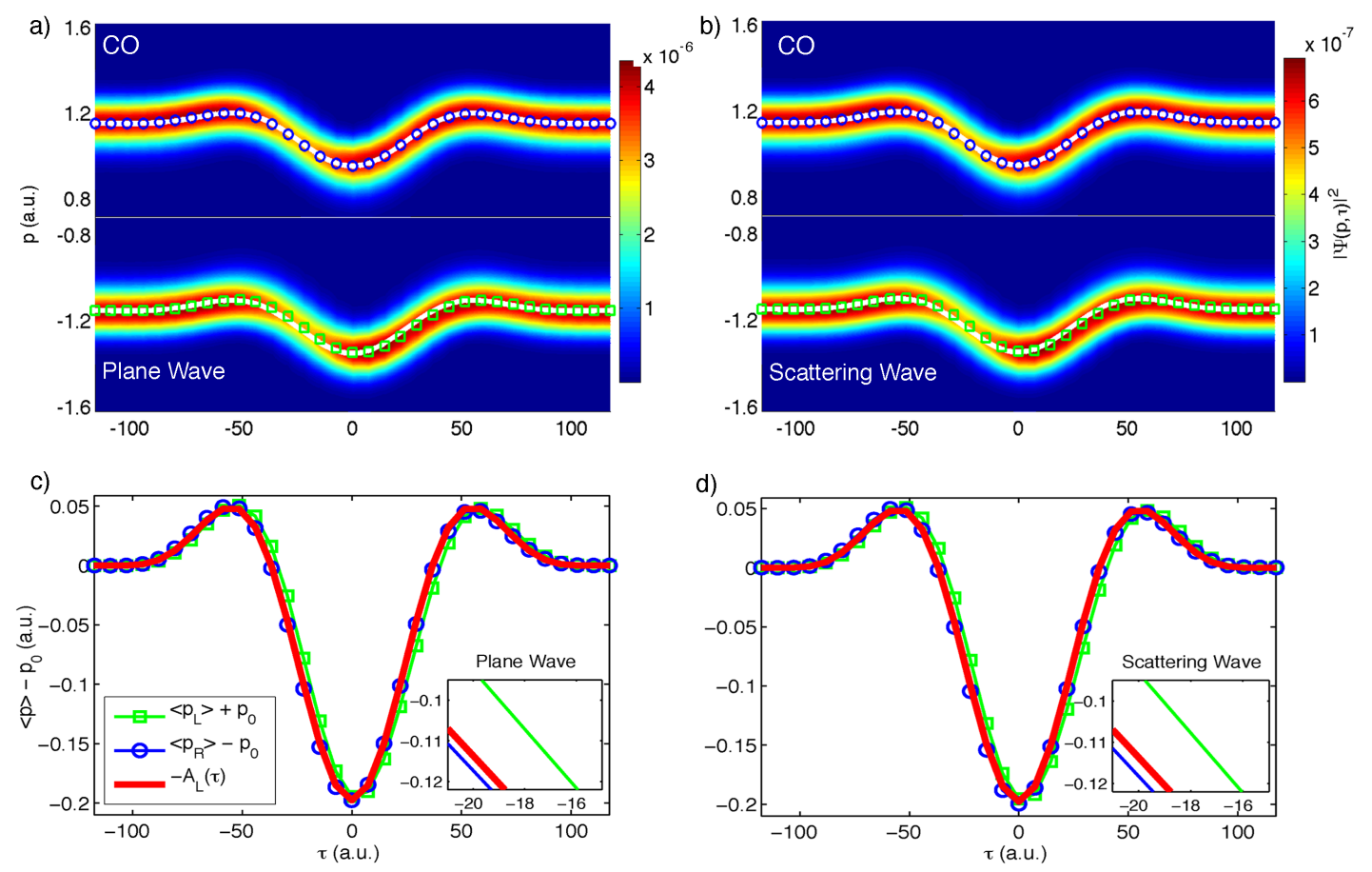

Figure 5.19: Streaking trace by $\mathbf{P W}$ and SW projections for the CO molecule. This figure shows the same as Fig. 5.18 in case of $\mathrm{CO}$ but the streaking traces are computed by the PW and SW projections.

In order to compare if the calculated streaking traces by $\mathrm{PW}$ and $\mathrm{SW}$ projections yield the same information about the stereo streaking time delay in the molecular case, we have computed them. Fig. 5.19a-b) show the comparison between the left-right streaking traces by projecting on $\mathrm{PW}$ and $\mathrm{SW}$ for the $\mathrm{CO}$ molecule system. As it is expected the amplitude shapes look similar for both cases but the absolute values differ each other. However, the shifted momentum expectation values are similar if PWs or SWs are used to compute these quantities (see Figs. 5.19c-d)). The inset graphs in c) and d) show a very similar stereo streaking time delay $\Delta t_{\mathrm{S}}^{(\mathrm{LR})} \approx-3.0$ a.u., if PWs or SWs are used to compute the streaking traces.

It is important to indicate that this result does not mean that the PW or SW yields the same final momentum. This result means that the same stereo streaking time delay between the left and right is recovered by using both PW and SW projections for the EWP with central energy $E_{0}=0.69$ a.u.

To test whether the streaking technique works in a broad range of XUV frequencies, 


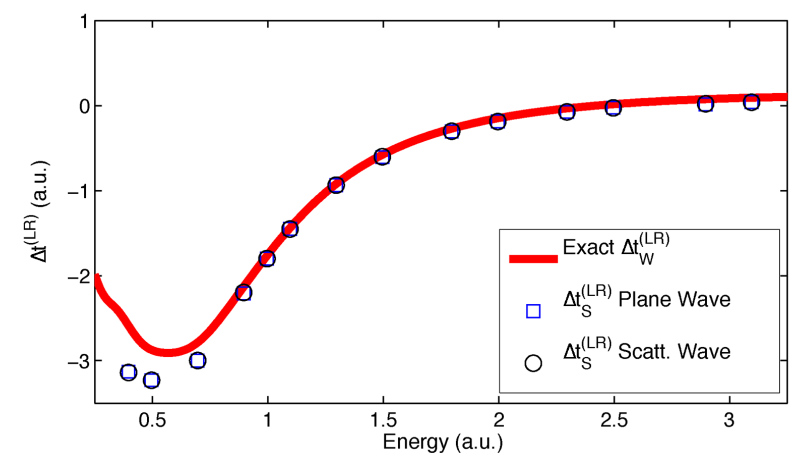

Figure 5.20: Stereo streaking time delay for the molecular case. The stereo streaking time delay as a function of the EWP central energy $E_{0}=\omega_{\mathrm{X}}-I_{p}$ by $\mathrm{PW}$ (blue squares) and SW (black circles) are compared to the exact stereo Wigner time delay $\Delta t_{\mathrm{W}}^{(\mathrm{LR})}$ computed via the derivative of the dipole phase.

we have calculated the streaking traces left-right by PW or SW projections for a set of XUV central frequencies between $0.90(24.5 \mathrm{eV})$ and 3.6 a.u. $(97.9 \mathrm{eV})$. The XUV attosecond peak intensity is fixed to $I_{\mathrm{X}}=5 \times 10^{12} \mathrm{~W} / \mathrm{cm}^{2}$, the FWHM are between 15 and 22 a.u., and the CEP is zero for all the attosecond pulses.

The results are depicted in Fig. 5.20. We find a very good agreement between the stereo time delay extracted by our stereo streaking method and the exact SWTD $\Delta t_{\mathrm{W}}^{(\mathrm{LR})}$ as obtained from the exact complex dipole transition matrix element and the TOF technique defined above.

This shows that the SWTD can be measured experimentally and provides a simple way to remove the CLC. The technique is very sensitive to the asymmetry of the molecular potential and is robust to laser parameter changes.

Furthermore, we address to the question how the stereo streaking time delay $\Delta t_{\mathrm{S}}^{(\mathrm{LR})}$ changes as a function of the XUV attosecond pulse FWHM and the peak amplitude of the IR electric field compared to the exact averaged $\operatorname{SWTD}\left\langle\Delta t_{\mathrm{W}}^{(\mathrm{LR})}\right\rangle$ within the energy bandwidth of the corresponding EWP.

We have numerically calculated the streaking traces fixing the central frequency $\omega_{\mathrm{X}}=1.5$ a.u., and the $\mathrm{CEP}=0$.

The results are shown in Fig. 5.21. We find that the SSTD changes as the FWHM increases and it is in good agreement with the exact averaged SWTD $\Delta t_{\mathrm{W}}^{(\mathrm{LR})}$. There is a deviation for small values of FWHM which are related to numerical errors.

The streaking time as a function of the electric field peak amplitude is shown in Fig. 5.21 and it is constant. This demonstrate that the CLC has been effectively eliminated from 

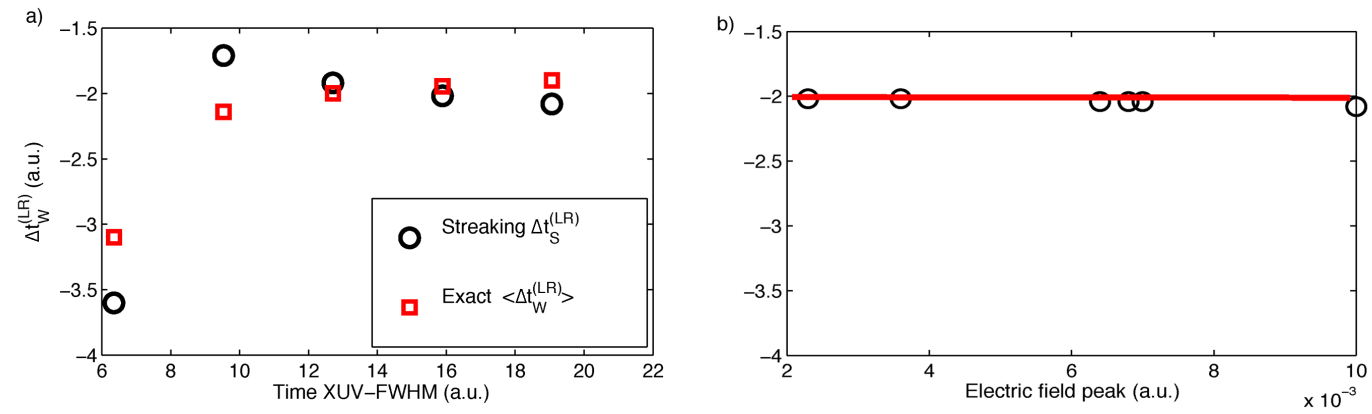

Figure 5.21: Analysis of the stereo streaking time delay. a) The SSTD (black circles) as a function of the XUV attosecond FWHM compared to the exact average of the stereo Wigner time delay $\left\langle\Delta t_{\mathrm{W}}^{(\mathrm{LR})}\right\rangle$ (red squares). b) The SSTD $\Delta t_{\mathrm{S}}^{(\mathrm{LR})}$ (black circles) as a function of the IR electric field peak amplitude $E_{\mathrm{L}, 0}$ compared to the exact average Wigner time delay (red line).

the measurement process.

For the analysis performed in this Section, we conclude that the stereo Wigner time delay can be measured by implementing the stereo attosecond streaking technique. We note that the problematic CLC is cancelled out by the stereo delay detection method and therefore the fundamental Wigner time delay or delay in photoemission is recovered. Thus, this attosecond stereo streaking method configures a powerful method to extract dynamical. In addition, structural information regarding the small asymmetric molecule can be recovered as well, i.e., the Wigner time delay dependence on the internuclear distance.

\subsection{Asymmetric molecule in three dimensions}

In the last Sections, it was demonstrated that the problematic CLC could be removed from the measurement process of the stereo time delay by using the stereo attosecond streaking method.

In the next Sections, we will show how to extend our 1D numerical model to a three dimensional asymmetric molecular potential formed by two nuclei. We show that the SWTD can be extracted by the attosecond streak camera in similar way as it was done in the $1 \mathrm{D}$ model.

In addition, we will show how the bound-bound electron dynamics could also play an important role in the measurement process of the stereo Wigner time delay. The 
induced couplings on the continuum-continuum or bound-bound states created by the probe IR laser pulse become increasingly important and complicate the detection of the Wigner time delay or the time delay in photoemission.

It is important to mention that previous theoretical predictions [162] have demonstrated that the interaction of an IR laser field can easily induce an electron boundbound dynamics depending of the energy structure of the molecule. Lötstedt et al. [162], have studied the ionization of the acetylene induced by an intense IR laser field and found that the ionization increases drastically when the internuclear distance of the $\mathrm{C}-\mathrm{H}$ bond is increased. In addition, they noted that a bound-bound electron dynamics is induced by the IR laser field during the interaction. Then, this bound-bound electron dynamics influences the ionization process. It is so due to the fact that the two possible channels can create a constructive or destructive coherent superposition and then, to affect the electron detection signal.

As we will see, the 3D model has a structure of orbitals where the energy gap between the HOMO and the upper excited orbital, is close to the photon energy of the IR laser field. This fact will show us that the stereo Wigner time delay in the photoemisson process could be difficult to extract from such a system by utilizing the streaking technique.

To perform the time evolution of a real 3D molecule, i.e., the $\mathrm{CO}$, including all the interactions of the system and the electron-electron correlation and nuclear motions, configures a very challenging problem from a computational viewpoint. For this reason, we will restrict ourselves to the SAE and we will consider that the nuclear motion is slower compared to the electronic one during the interaction with the ultrashort femtosecond and attosecond laser pulses.

Furthermore, we use a linearly polarized laser field along the oriented molecular axis. This configuration simplifies the problem in the sense that cylindrical symmetry can be used in order to simulate the interactions of the system, i.e., the effective potential will depend only on the coordinates $(z, \rho)$ and not on the azimuthal angle $\varphi$. This suggests that the time evolution of the electronic full wavefunction will not rely upon the magnetic quantum number $m=0$ and the main propagation of the wavefunction will be along the coordinates $z$ and $\rho$, while keeping the 3D nature of the problem. We will follow the numerical implementation given in the Chapter 2 to integrate the TDSE in cylindrical coordinates.

In order to test the ideas propose here, through next subsections we will use the cylindrical symmetry to solve numerically the TISE and TDSE for an orientated molecular system formed by two centers. 


\subsubsection{Definition of the system}

In this Section, we describe the properties of the asymmetric molecular Coulomb potential and the calculation of the $5 \sigma$ orbital, that can be associated to a real CO molecule.

The Hamiltonian $H_{0}=\frac{p_{\rho}^{2}}{2}+\frac{p_{z}^{2}}{2}+V_{0}(\rho, z)$ describes the interactions of the system without the presentce of laser field in cylindrical coordinates. We choose a Coulomb potential $V_{0}(\rho, z)$ to mimic an asymmetry molecule with two centers which allow us to describe the asymptotic coulombic behavior of the real molecular system:

$$
V_{0}(\rho, z)=-\frac{Z_{1}}{\sqrt{\left(z+\frac{R}{2}\right)^{2}+\rho^{2}}}-\frac{Z_{2}}{\sqrt{\left(z-\frac{R}{2}\right)^{2}+\rho^{2}}},
$$

where $Z_{1}$ and $Z_{2}$ are the charges which are located along the $z$-axis. The coordinate $z$ denotes the main cylindrical axis and $\rho=\sqrt{x^{2}+y^{2}}$ the polar radial coordinate in the plane $x$ and $y$.

We compute the five first bound states of the asymmetric potential Eq. (5.14) by solving the TISE via the imaginary time propagation described in Section 2.2.1.4. The imaginary time step is $\Delta t=-0.04 i$ and the grid size for $z$ and $\rho$ is $L_{z}=350$ a.u., and $L_{\rho}=150$ a.u., with a spacing $\Delta z=\Delta \rho=0.2$ a.u., respectively.

The charges $\left(Z_{1}, Z_{2}\right)$ are chosen such as they match as closely as possible the ionization potential $I_{p}$ of the HOMO orbital for a real CO molecule, and the Coulomb $-Z / r$ (with $r=\sqrt{z^{2}+\rho^{2}}$ ) asymptotic $r \rightarrow \infty$ behavior of the potential $V_{0}(z, \rho)$. We have found that the best choice are $Z_{1}=0.7$ and $Z_{2}=2.3$ with a total charge $Z=Z_{1}+Z_{2}$. The internuclear distance is fixed at $R=2.2$ a.u. (1.16 $\AA$ ). With these parameters we obtain a $5 \sigma$ orbital with $I_{p}=0.58$ a.u., $(15.6 \mathrm{eV})$. These data, $R$ and $I_{p}$ are close to the experimental values [161] for the HOMO of the CO molecule.

Our cylindrical approach allows us to represent the position electron distribution $|\Psi(\rho, z)|^{2}$ as a function of the spatial cartesian coordinates $(x, y, z)$. Fig. 5.22 shows an iso-volumetric visualization of the asymmetric potential, $V_{0}(\rho, z)$ and the HOMO orbital $5 \sigma$. We find that this molecular orbital is asymmetric and has two structural nodes similar to the real CO [163].

Note that the larger nuclear charge, $Z_{2}$, is located at $z=+R / 2$ (on the left with respect to the $z=0$ ) in the asymmetric coulombic potential ${ }^{1}$. However, the probability density shows a smaller iso-volume region about $Z_{2}$ compared to the electronic density around $Z_{1}$. This counter-intuitive result means that more electron density is located

\footnotetext{
${ }^{1}$ Note that while in Section 5.3 the charge $Z_{1}$ is larger than $Z_{2}$, in this Section and in the following ones, the charge $Z_{2}$ is larger than $Z_{1}$. This only changes the nuclei positions for "C" and "O".
} 


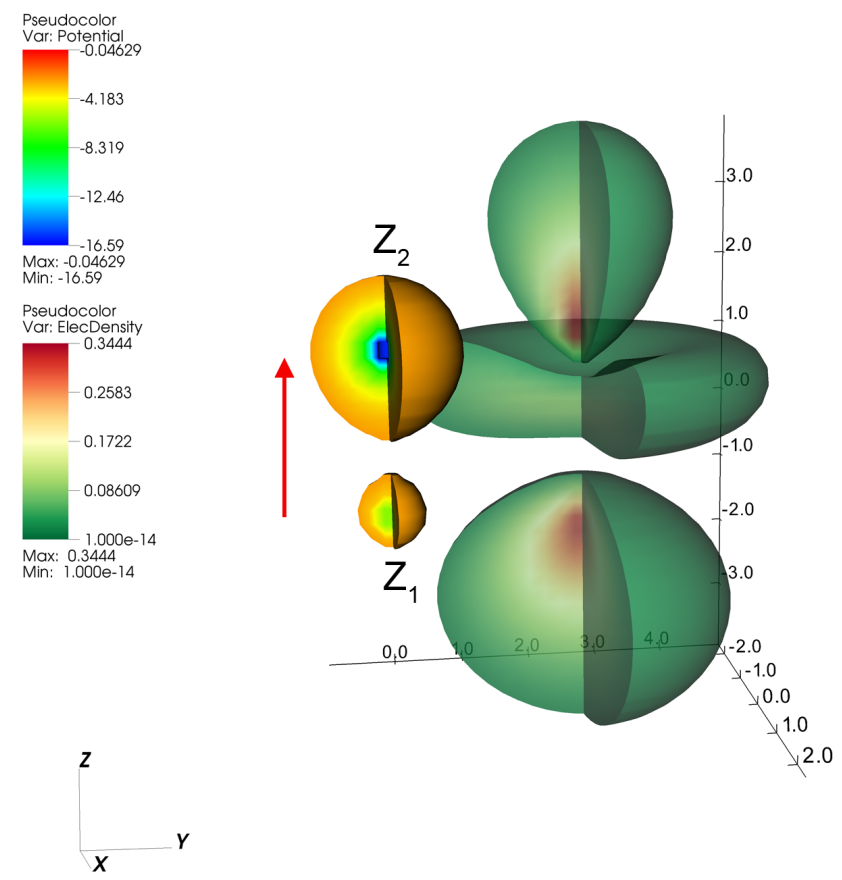

Figure 5.22: Oriented asymmetric molecular system in 3D. The cartesian 3D visualization of the asymmetric coulombic potential (blue-red color scale) and the orbital $5 \sigma$ (green-sangria color scale) are depicted as a function of the position $(x, y, z) . Z_{1}$ and $Z_{2}$ denote the location of the nuclei charges for the "C" and "O", respectively. Red arrow points out the direction of the static dipole and the larger nucleus charge. The solid iso-volume of the electron density is shifted along $y$-axis to distinguish it from the potential. The potential and orbital iso-volumes are cut around an angle of $120^{\circ}$ which allow us to view the change of the asymmetric potential and the electron density for different spatial volumes. 
around the core with less nuclear charge $Z_{1}$. The static dipole of the system is pointing out to the larger nuclear charge $Z_{2}$ as it is indicated in the Fig. 5.22 by a red arrow. This result is similar to the one obtained in reference [163].

The SW construction for a molecular asymmetric potential in 3D is a very demanding task, computationally speaking. For this reason, the calculation of the complex bound-free dipole transition matrix element is difficult and, as a consequence, the exact computation of the Wigner time delay. As an alternative way in the next Section we will compute the SWTD by employing the TOF method.

\subsubsection{Stereo TOF: 3D results}

We have defined the TOF method to extract the stereo time delay in photoemission by tracking in time the position expectation value $\langle z\rangle$ in $1 \mathrm{D}$, however, in $3 \mathrm{D}$ the situation is rather different. Due to the asymmetric nature of the $5 \sigma$ orbital and considering the free EWP is emitted now on all directions, the electron motion is not only restricted to a line anymore.

Thus, we modify our definition of the semi-classical analysis in 1D to an "averaged" electron trajectory. We calculate the position expectation value on the left $\left\langle z_{\mathrm{L}}\right\rangle$ and on the right $\left\langle z_{\mathrm{R}}\right\rangle$ around a small detection cylinder of radius $\rho_{d}$ and with respect to a mean position $\left\langle z_{5 \sigma}\right\rangle$ of the $5 \sigma$ orbital:

$$
\left\langle z_{\mathrm{L} / \mathrm{R}}\right\rangle-\left\langle z_{5 \sigma}\right\rangle=\int_{\mp z_{0}}^{\mp \infty} z d z \int_{0}^{\rho_{d}} \rho\left|\Psi_{c}(\rho, z, t)\right|^{2} d \rho-\left\langle z_{5 \sigma}\right\rangle
$$

where $\rho_{d}=1.0$ is the radius of the small cylinder, $z_{0}=0$, the initial position to calculate the expectation value, $\left\langle z_{5 \sigma}\right\rangle=-0.5552$ a.u., and $\Psi_{c}(\rho, z, t)$ is the field-free complex wavefunction of the system.

Classically, we assume that the electron appears in the continuum at $\left\langle z_{5 \sigma}\right\rangle$ and from this position we track the trajectories on either side. Then, we fix symmetric positions on the left-right trajectories $\left|\left\langle z_{\mathrm{L}}\right\rangle\right|=\left\langle z_{\mathrm{R}}\right\rangle=z_{d}$ and by interpolating on the time axis $t$ to each trajectory, we obtain the TOF $\Delta t_{\mathrm{TOF}}^{(\mathrm{L})}$ and $\Delta t_{\mathrm{TOF}}^{(\mathrm{R})}$ that each of the EWPs spend to reach either position in the same way that was explained in Section 5.3.3. The difference between both times $\Delta t_{\mathrm{TOF}}^{(\mathrm{LR})}=\Delta t_{\mathrm{TOF}}^{(\mathrm{L})}-\Delta t_{\mathrm{TOF}}^{(\mathrm{R})}$ will define the stereo time delay.

To compute the time-space evolution of the electronic wavefunction $\Psi_{c}(\rho, z, t)$, we numerically integrate the 3D TDSE in cylindrical coordinates via the Crank-Nicolson 
algorithm. The Hamiltonian of the system $H=\frac{1}{2}\left[p_{z}+A_{\mathrm{X}}(t)\right]^{2}+\frac{p_{\rho}^{2}}{2}+V_{0}(\rho, z)$ describes all the interactions between the XUV attosecond pulse and our oriented asymmetric molecular model. The vector potential of the XUV pulse is denoted by $A_{\mathrm{X}}(t)$ which is linearly polarized along the $z$-axis. We have obtained the continuum wave packet wavefunction $\Psi_{c}(\rho, z, t)$ by masking the bound states from $\Psi(\rho, z, t)$.

a)

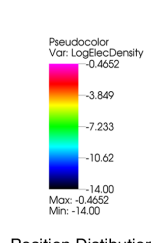

Position Distibution

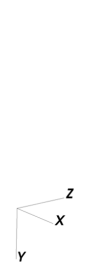

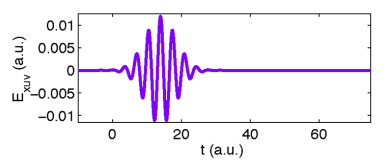

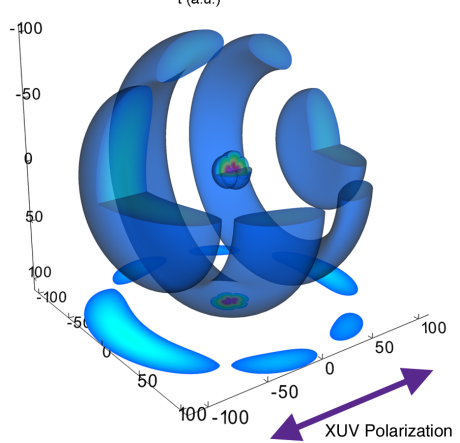

b)

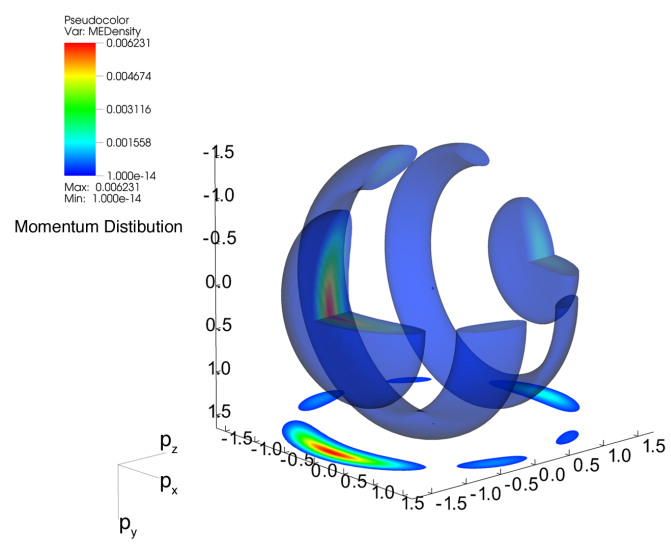

Figure 5.23: Molecular attosecond XUV electron wavepacket in 3D. a) The iso-volume of the full electron density for the bound and free wavefucntion as a function of the position coordinates $(x, y, z)$ after the XUV attosecond interaction with the asymmetric molecular system. A cut in the position distribution at $y=0$ is shown in the plane $(x, z)$. The inset graph shows the XUV attosecond pulse in violet. b) The final momentum electron distribution as a function of the momentum coordinates $\left(p_{x}, p_{y}, p_{z}\right)$. The cut at the momentum $p_{y}=0$ is depicted as well in the momentum plane $\left(p_{x}, p_{z}\right)$. The attosecond XUV parameters are: frequency $\omega_{\mathrm{X}}=1.8$ a.u., peak intensity $I_{0}=5.0 \times 10^{12} \mathrm{~W} / \mathrm{cm}^{2}, \mathrm{CEP}=\frac{\pi}{2}$ and its envelope is a gaussian function with FWHM $=10.6$ a.u.

The grid parameters used to solve the TDSE are the same as the ones used in the last Section 5.4.1. The time step is $\Delta t=0.04$ a.u.

Fig. 5.23 shows the 3D final position and momentum distributions of the emitted EWP by a SAP. We find that the iso-volume of the bound $\left|\Psi_{b}(x, y, z, t)\right|^{2}$ and electron position distributions $\left|\Psi_{c}(x, y, z, t)\right|^{2}$ are asymmetric in a) as it is expected. Note that the free electron density has three nodes. These information come from the structural shape of the orbital $5 \sigma$ and the coupling made by the XUV attosecond pulse between the HOMO orbital and the continuum states. We can also appreciate that a cut of the 
position electron density (at $y=0$ ) along the plane $(x, z)$ gives the same information about the asymmetry and the structural nodes of the molecule.

The 3D visualization of the momentum distribution and its cut along the plane $\left(p_{x}, p_{z}\right)$ are shown in Fig. 5.23b). This momentum distribution $\left|\Psi_{c}\left(p_{x}, p_{y}, p_{z}\right)\right|^{2}$ is built by projecting the continuum wave packet on PW. The asymmetry in the momentum distribution is also visible in the iso-volume. The center momentum of the spherical cap is at $p_{0}=1.56$ a.u., which indicates that the asymmetry follows the charge distribution.

a)

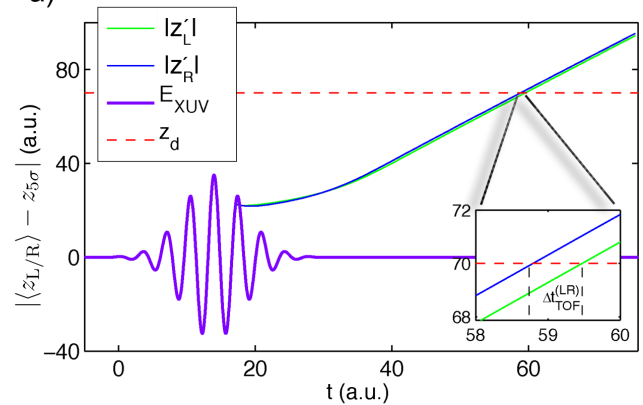

b)

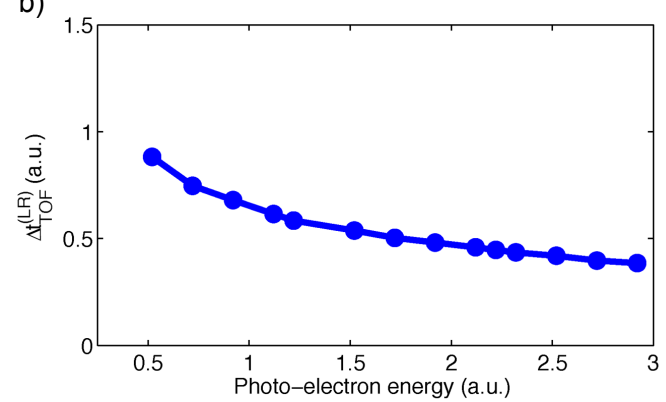

Figure 5.24: Stereo Winger time delay by using the TOF method: 3D results. a) The relative expectation position values on the left $\left|z_{\mathrm{L}}^{\prime}\right|=\left|\left\langle z_{\mathrm{L}}\right\rangle-z_{5 \sigma}\right|$ (green line) and right $\left|z_{\mathrm{R}}^{\prime}\right|=\left|\left\langle z_{\mathrm{R}}\right\rangle-z_{5 \sigma}\right|$ (blue line) as a function of time $t$ of EWPs emitted on either side along the $z$-axis. The violet line denotes the XUV attosecond pulse electric field, which has the same parameters that one shown in Fig. 5.23. The horizontal red dashed line indicates the symmetric detection position $z_{d}=70$ a.u., used to determine the TOF on the left-right and the stereo photoemission time delay $\Delta t_{\mathrm{TOF}}^{(\mathrm{LR})}=0.67 \mathrm{a} . \mathrm{u}$, which is depicted in the inset plot. b) The stereo photoemission time delay computed by using the TOF, is drawn as a function of photoelectron energy $E_{0}=\omega_{\mathrm{X}}-I_{p}$.

Fig. 5.24a) depicts the absolute value of the position expectation $\left|z_{\mathrm{L} / \mathrm{R}}^{\prime}\right|=\mid\left\langle z_{\mathrm{L} / \mathrm{R}}\right\rangle-$ $z_{5 \sigma} \mid$ along the $z$-axis including a small portion of the EWPs emitted on the left and right according to Eq. (5.15).

The XUV attosecond pulse parameters utilized are the same as the ones we used in Fig. 5.23. The stereo time delay in photoemission extracted by the TOF method yields a value of $\Delta t_{\mathrm{TOF}}^{(\mathrm{LR})}=0.67 \mathrm{a} . \mathrm{u}$. This result suggests that the EWP emitted on the right is born before than the emitted on the left. From a qualitative and classical point of view an electron which starts its motion on the direction of a deeper potential will experiment a larger acceleration compared to one that past through a potential which is not so deep. This is the case of our 3D potential model and consequently the positive value in the time asymmetry $\Delta t_{\mathrm{TOF}}^{(\mathrm{LR})}$ can be understood. 
The stereo time delay in photoemission as a function of the photoelectron energy is shown in Fig. 5.24b). The XUV attosecond parameters used in these simulation are, central frequencies $1.1-3.5$ a.u., peak intensities are fixed to $I_{0}=5 \times 10^{12} \mathrm{~W} / \mathrm{cm}^{2}$, $\mathrm{CEP}=\frac{\pi}{2}$ and the FWHM are between $12-18$ a.u.

The stereo TOF delay shows a dependence with the photoelectron energy which is not the same as in the 1D model. First of all, the core positions in 1D model are opposed and secondly the potential well is a soft-core Coulomb one which takes into account a neutral molecule charge. On the contrary, in the present 3D case the total nuclear charge corresponds to a molecular ion.

So far our 3D asymmetric molecular study suggests that there is an asymmetry in the time delay to the EWPs emitted on the $z$-left direction compared to the emitted on the right. The Fig. 5.24a) also suggests that EWPs trajectories $\left|z_{\mathrm{L} / \mathrm{R}}^{\prime}\right|$ are parallel to each other. Thus, we can conclude that the stereo time delay in photoemission $\Delta t_{\mathrm{TOF}}^{(\mathrm{LR})}$ is the same for all the fixed detection positions.

As it was indicated in Section 5.3.3 this method is not suitable for an experiment in the lab. In the next Section we will address how this stereo Wigner time delay can be measured by employing attosecond science tools.

\subsubsection{Stereo streaking technique under the three dimensional TDSE}

In this Section we will employ the streaking technique to extract information about the stereo Wigner time delay in the one-photon ionization process from the HOMO of the 3D asymmetric molecule model as in Section 5.2.2.

The streaking effect to the asymmetric molecule will be obtained by the numerical solution of the 3D TDSE. Then, we introduce the streaking trace to extract the stereo streaking time delay in photoemission taking advantage of the momentum expectation values for electrons emitted on the left and on the right. We will find and point out important issues related with the measurement process needed to recover the stereo delay in photoemission from the HOMO orbital by the attosecond streaking technique.

The streaking effect is mainly defined by the momentum shift $\Delta p$ that an EWP can experiment when it is ionized from an atom or molecule by a single XUV attosecond pulse in presence of a weak IR laser field. According to our previous Section 5.2.2 this momentum shift $\Delta p_{z}=-A_{\mathrm{L}}(\tau)$ is given by the negative value of the vector potential $A_{\mathrm{L}}(\tau)$ along the IR polarization axis at the ionization time delay $\tau$. This time delay $\tau$ is measured with respect to the maximum of the IR laser field and the attosecond pulse envelopes. Then, the streaking trace is defined by the electron momentum or energy 
distribution as a function of the scanned time delay $\tau$.

In order to obtain the stereo streaking time delay from the attosecond streaking trace, we solve numerically the TDSE in 3D under the streaking conditions. By taking the Fourier analysis on the left and right momentum expectation values, we will be able to obtain the stereo streaking time delay. However, first, we solve the TDSE to show the 3D streaking effect and to visualize the 3D asymmetry of the EWP momentum distribution.

The Hamiltonian which describes the interactions of the molecule under the SAE approach with these two pump-probe pulses, i.e. the XUV attosecond and the IR laser, reads $H=\frac{p_{\rho}^{2}}{2}+\frac{1}{2}\left[p_{z}+A_{\mathrm{L}}(t)+A_{\mathrm{X}}(t-\tau)\right]^{2}+V_{0}(\rho, z)$. Here, the $A_{\mathrm{L}}(t)$ and $A_{\mathrm{X}}(t-\tau)$ are the vector potentials of the IR laser field and the XUV attosecond pulse, respectively. They are linearly polarized along the $z$-axis. The time delay $\tau$ is the time between the maximum of the IR laser field and the XUV pulse envelope. Positive (negative) time delay means the IR laser arrives before (after) than the XUV attosecond pulse.

Fig. 5.25 compares the 3D momentum distribution for a single EWP emitted by a single XUV attosecond pulse a), to a different EWPs emitted when the XUV arrives at the zero b) and maximum c) of the IR laser field vector potential $A_{\mathrm{L}}(t)$. We find out that the EWP momentum distribution is not shifted in the case of b) meanwhile in c) it is along the polarization axis $(z)$ of the IR laser field. Then, our numerical results show that the EWP is momentum shifted by a small negative value of the IR laser field vector potential $-A_{\mathrm{L}}\left(t_{i}\right)$ at the ionization time $t_{i}=\tau+\Delta t_{\mathrm{S}}$. This is nothing but the streaking effect. Note that this shifted momentum distribution is strongly asymmetric and the EWP amplitude is larger along the negative $p_{z}$-axis (see the iso-volume of Fig. 5.25c)).

To extract the time delay in photoemission by using the streaking technique, we compute the streaking traces to the EWPs emitted on either side. We scan on the time delay $\tau$ the 3D final momentum distribution $p_{\rho}\left|\Psi_{c}\left(p_{\rho}, p_{z}, \tau\right)\right|^{2}$ and then the streaking trace $\mathrm{S}_{\mathrm{e}}\left(p_{z}, \tau\right)$ is calculated by integrating the full 3D momentum distribution about a small angle of $\theta_{0}=10^{\circ}$ for electrons emitted with negative and positive momenta along $p_{z}$-axis. The angle $\theta$ is defined by $\tan \theta=\frac{p_{\rho}}{p_{z}}$, where $p_{\rho}$ and $p_{z}$ are the $z$ and $\rho$ axis in the momentum plane.

Fig. 5.26 shows the streaking traces for electrons emitted with negative and positive momenta from the $5 \sigma$ orbital. The attosecond XUV frequency is $\omega_{\mathrm{X}}=2.0$ a.u., its peak intensity $I_{\mathrm{X}}=5.0 \times 10^{12} \mathrm{~W} / \mathrm{cm}^{2}$, the $\mathrm{FWHM}=9.48$ a.u. $(229 \mathrm{as})$, and the $\mathrm{CEP}=\frac{\pi}{2}$. The IR laser field parameters are the same as those used in Fig. 5.25.

The left-right momentum streaking traces $\mathrm{S}_{\mathrm{e}}\left(p_{z}, \tau\right)$ show clearly an asymmetry (see the 
a)

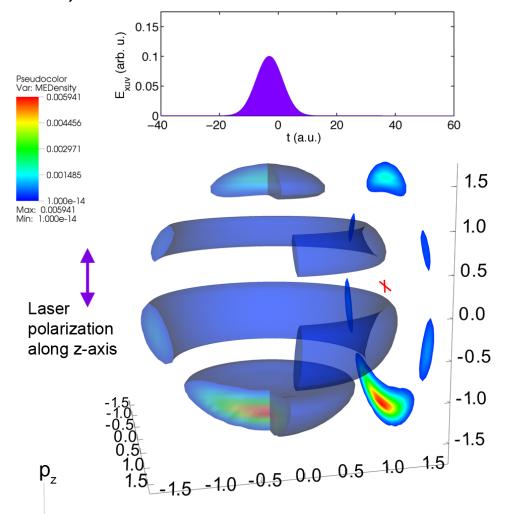

b)

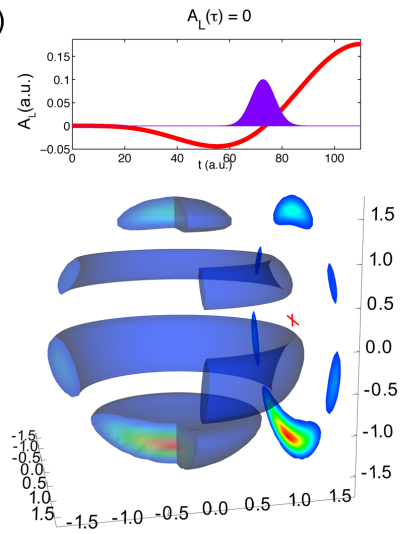

c)
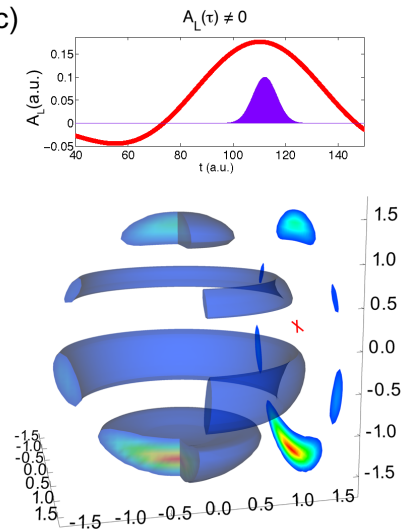

$p_{x} p_{y}$

Figure 5.25: Attosecond streaking effect obtained by 3D TDSE. 3D momentum distribution $\left|\Psi_{c}\left(p_{x}, p_{y}, p_{z}\right)\right|^{2}$ and its cut along the plane $\left(p_{x}, p_{z}\right)$ at $p_{y}=0$, are shown in a color iso-volume and surface, when an EWP is ionized via a) a single XUV attosecond pulse, b) and c) the mix of "two colors", i.e., an XUV and a weak IR laser field. In the case of b) and c) the instantaneous vector potential $A_{\mathrm{L}}\left(t_{a}\right)$ (or $A(\tau)$ ), at the XUV arrival time $t_{a}=74$ a.u. $\left(\tau \sim-T_{0} / 4\right)$, is zero $A_{\mathrm{L}}(\tau)=A_{\mathrm{L}}\left(t_{a}\right)=0$, and different than zero $A_{\mathrm{L}}\left(t_{a}\right) \neq 0$, at the $t_{a} \approx 110$ a.u. $(\tau=0)$, respectively. The electric field envelope $E_{\mathrm{X}}(t)$ of the XUV attosecond pulse (violet area) and the IR laser field vector potential $A_{\mathrm{L}}(t)$ (red curve) are depicted on the top of each corresponding panel. The full momentum distribution in c) shows clearly the streaking effect: the momentum shift $\Delta p_{z}=-A_{\mathrm{L}}(\tau)=A_{\mathrm{L}}\left(t_{a}\right) \approx-0.15$ a.u. The XUV attosecond pulse parameters of the simulations are the same as in Fig. 5.23 and the IR laser field peak intensity is $2.0 \times 10^{12} \mathrm{~W} / \mathrm{cm}^{2}$, the $\mathrm{CEP}=0$, its central frequency $\omega_{0}=0.057$ a.u., $(800 \mathrm{~nm})$ and time duration two cycles under a sine-squared envelope. 

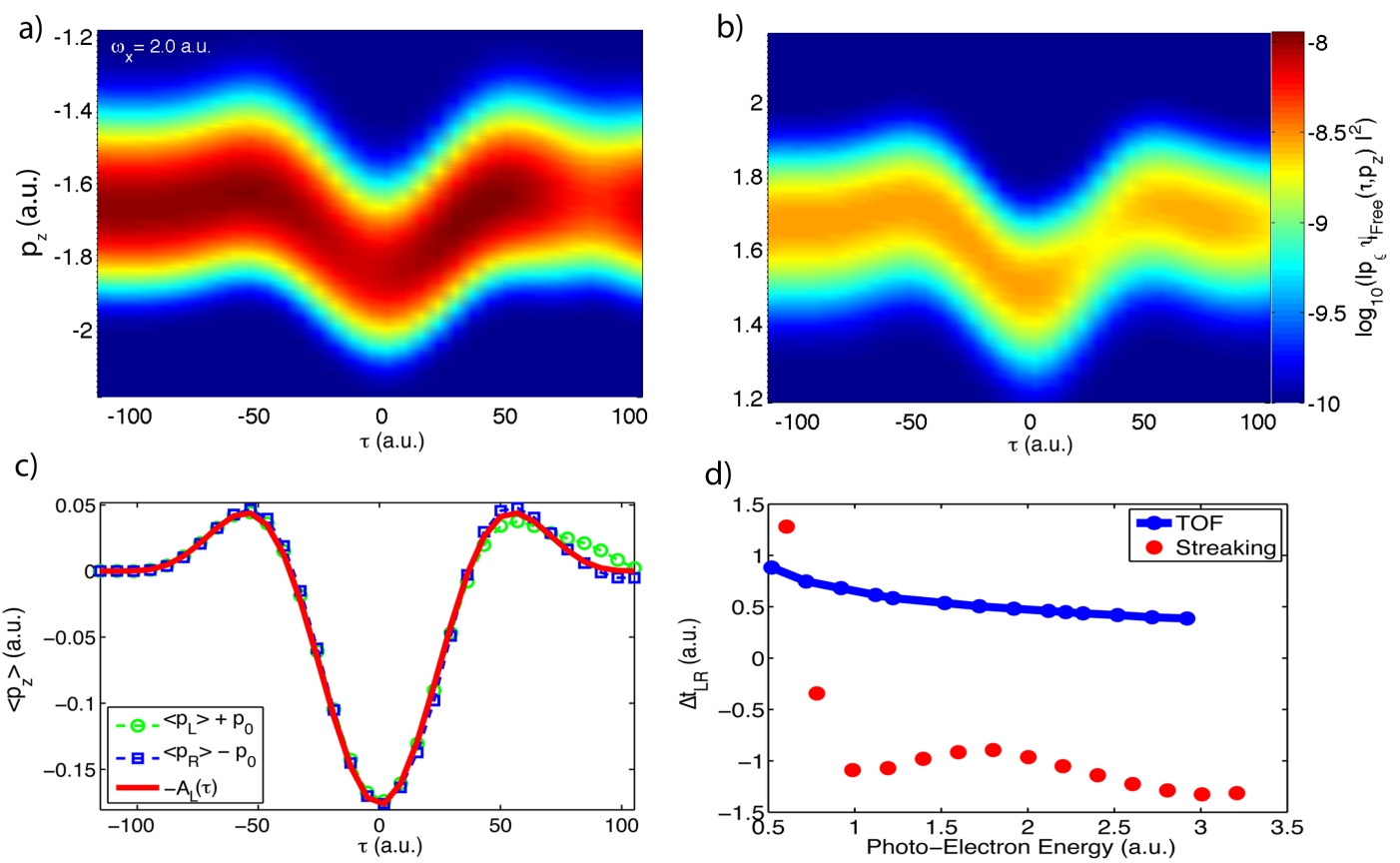

Figure 5.26: Streaking traces: 3D TDSE calculations for the HOMO. The graphs a) and $\mathrm{b}$ ) show the integrated $3 \mathrm{D}$ momentum distributions $\mathrm{S}_{\mathrm{e}}\left(p_{z}, \tau\right)$ for the photoelectrons with negative and positive momentum as a function of the time delay $\tau$. c) The momentum expectation values left (green dashed line with circles) and right (blue dashed line with circles), and the negative values of the vector potential $A_{\mathrm{L}}(\tau)$ of the IR laser field. d) The plot shows the stereo TOF delay $\Delta t_{\mathrm{TOF}}^{(\mathrm{LR})}$ (blue line with circles), and the corresponding stereo streaking time delay $\Delta t_{\mathrm{S}}^{(\mathrm{LR})}$ as a function of the photoelectron energy $E_{0}$. 
Figs. $5.26 \mathrm{a}-\mathrm{b})$ ) along the momentum $p_{z}$-axis. The electron population ionized with negative momentum is larger than the one with positive momentum, as it is expected. We note that an strong asymmetry in $\mathrm{S}_{\mathrm{e}}\left(p_{z}, \tau\right)$ is found along the time delay $\tau$-axis in Figs. $5.26 \mathrm{a}-\mathrm{b})$. We could wonder what is the meaning of such an asymmetry? This question will be addressed after the evaluation of the fact that if the attosecond streaking technique works to extract the stereo time delay in photoemission.

In order to extract the stereo streaking time delay $\Delta t_{\mathrm{S}}^{(\mathrm{LR})}$, we follow similar steps as indicated in Section 5.3.4. The momentum expectation values on the left-right $\left\langle p_{z,(\mathrm{~L} / \mathrm{R})}\right\rangle$ are calculated as follows:

$$
\left\langle p_{z,(\mathrm{~L} / \mathrm{R})}\right\rangle=\frac{\int_{0}^{\mp p_{z_{f}}} d p_{z} p_{z} \mathrm{~S}_{\mathrm{e}}\left(p_{z}, \tau\right)}{\int_{0}^{\mp p_{z_{f}}} d p_{z} \mathrm{~S}_{\mathrm{e}}\left(p_{z}, \tau\right)},
$$

and from the phase Fourier analysis (see Sec. 5.3.4) of those quantities the stereo streaking time delay $\Delta t_{\mathrm{S}}^{(\mathrm{LR})}$ can be extracted.

We subtract the center momentum $p_{0}$ to $\left\langle p_{z,(\mathrm{~L} / \mathrm{R})}\right\rangle$ and depict the results as a function of the time delay $\tau$ in Fig. 5.26c). We compare those left-right momentum values to the negative one of the IR laser field vector potential $-A_{\mathrm{L}}(\tau)$. This comparison shows a small shift between the left-right momentum expectation values and the IR laser field vector potential. In addition, we note that these momentum values $\left\langle p_{z,(\mathrm{~L} / \mathrm{R})}\right\rangle \pm p_{0}$ have an "extra oscillation" around a time delay $\tau>50$ a.u., this is contrast to our 1D model.

Although such oscillation on the expectation momentum values can distort the streak time delay, we employ the Fourier analysis to recover it anyways. We observe that, while the extracted stereo streaking time delay $\Delta t_{\mathrm{S}}^{(\mathrm{LR})}$ from the left-right momentum expectation values yields -0.98 a.u., the exact stereo TOF delay gives $\Delta t_{\mathrm{TOF}}^{(\mathrm{LR})}=0.56$ a.u. Then, we can not find agreement between the streak and TOF methods.

To test a broader range in the data of the extracted stereo streaking time, we calculate the streaking traces for different XUV attosecond pulses with central frequencies $\omega_{\mathrm{X}}=1.2-3.8$ a.u. We retrieve the stereo streaking time delay in photoemission $\Delta t_{\mathrm{S}}^{(\mathrm{LR})}$, as a function of the central EWP energy, $E_{0}=\omega_{\mathrm{X}}-I_{p}$ and depict those results on Fig. 5.26d). We can not find any agreement between the stereo streaking time delay and the expected stereo TOF delay as it is in the 1D model.

These latest results suggest that something else is happening in our 3D model, in 
particular with the HOMO for the AM, which can not allow us to retrieve the stereo Wigner delay by using the streaking technique. One can wonder if this stereo streaking method works or not to extract the stereo time delay and where the distorted time-delay asymmetry on the momentum distributions (see Figs. 5.26a-b)) is coming from.

To address these questions, first we will change the initial bound state and retrieve the stereo streaking time related to another state. Second, we will go back to the HOMO and compute the populations for the first five bound states of our asymmetric potential as a function of the time while the IR laser field is acting alone on the system. It is so because we have found that the energy gap between the HOMO and first upper excited state is close to photon energy of the IR laser field.

A way to prove if the streaking method works for measuring the stereo Wigner time delay is to change the initial bound HOMO state to another one, such as the HOMO-1, that has less energy than the $5 \sigma$. By solving the 3D-TDSE, we have computed the streaking traces on the left and right for the HOMO-1.

Figs. 5.27a) and b) show the numerical results for the left and right calculated streaking traces.

These momentum distributions were computed by integrating the full 3D electron momentum distribution over a small angle of $10^{\circ}$ between $p_{\rho}$ and $p_{z}$ as in Fig. 5.26. Note that the population depicted on the streaking trace for electrons emitted on the left is less than the one for electrons on the right. This result is opposite to the HOMO one (see Figs. 5.26a-b)) and reflects the different symmetry of the HOMO-1.

Once we obtain the results of both streaking traces, the left and right momentum expectation values are calculated and compared to the vector potential of the IR laser field. The results are shown in Fig. 5.27c). It is important to point out that we do not find the same small extra oscillation on the momentum expectation values as in Fig. 5.26c) for time delays $\tau>0$. These left and right momenta show a very well defined time shift with respect to the vector potential $-A_{\mathrm{L}}(\tau)$ of the IR laser field.

By the Fourier method, we find that the stereo streaking time delay is $\Delta t_{\mathrm{S}}^{(\mathrm{LR})}=-1.3$ a.u. We also calculate the stereo time delay by the TOF $\Delta t_{\mathrm{TOF}}^{(\mathrm{LR})}$ method for the HOMO-1 and using the same attosecond pulse as in the streaking traces. We have found that the value of the stereo TOF delay is $\Delta t_{\mathrm{TOF}}^{(\mathrm{LR})}=-1.37$ a.u., which is in very good agreement with the stereo streaking time delay $\Delta t_{\mathrm{S}}^{(\mathrm{LR})}$. We have extracted the same conclusion from our 1D model.

From these results we can conclude, first, that the stereo streaking technique works reasonably well to extract information of the stereo Wigner time delay for the HOMO1 and, second, the streaking technique is not able to extract the stereo Wigner time 

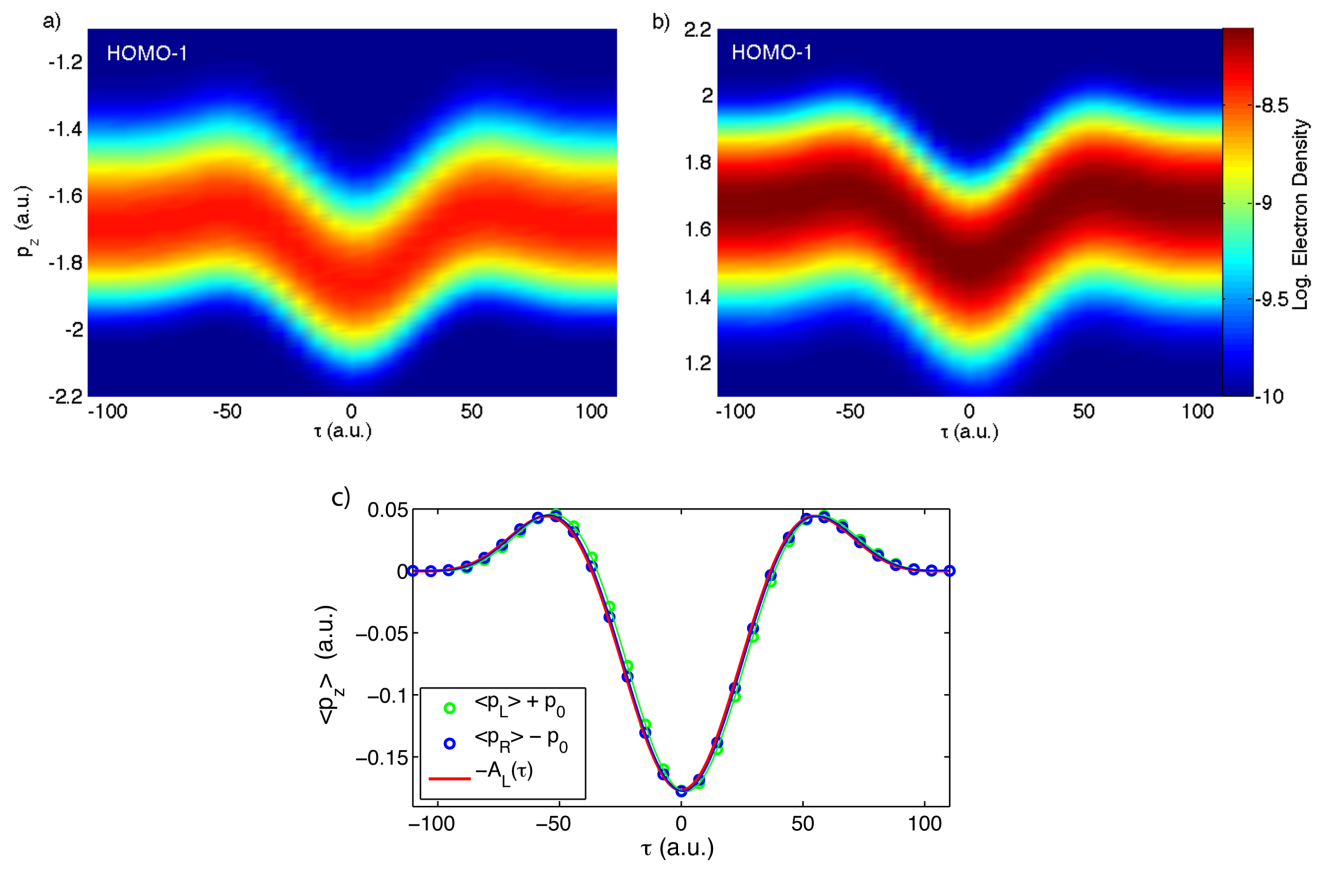

Figure 5.27: Streaking traces for the HOMO-1. a-b) Left and right momentum distribution of the continuum EWP as a function of the time delay $\tau$ when the ionized photoelectrons come from the first bound state with less energy than the $5 \sigma$ orbital (HOMO- 1 ). The color scale depicts the logarithm of the momentum distribution or electron density. c) Momentum expectation values for electrons emitted on the left (green circles) and right (blue circles), $\left\langle p_{\mathrm{L} / \mathrm{R}}\right\rangle \pm p_{0}$, where $p_{0}=\sqrt{2\left(\omega_{\mathrm{X}}-I_{\mathrm{p}}\right)}$. Red solid line depicts the vector potential negative $-A_{\mathrm{L}}(\tau)$ of the IR laser field. The IR-laser parameters are the same as in Fig. 5.26, and the XUV attosecond central frequency is $\omega_{\mathrm{X}}=2.3$ a.u., its peak intensity $I_{\mathrm{X}}=5 \times 10^{12} \mathrm{~W} / \mathrm{cm}^{2}$, its envelope is a gaussian with $\mathrm{FWHM}=250$ as, and its $\mathrm{CEP}=\frac{\pi}{2}$. 
delay in the case of the HOMO. Furthermore, according to the results for the HOMO1 , we have verified that our stereo streaking method works very well to extract the asymmetric time delay in photoemission and that this asymmetry is not an artefact caused by the dimensionality.

Here, two natural questions arise, namely, i) why the streaking technique is not able to extract the stereo Wigner time delay in case of the HOMO and it is so in the case of HOMO-1. ii) Where it is coming from the strong asymmetry along the time delay $\tau$-axis in the attosecond streaking traces case of HOMO (see Fig. 5.26a) or b)).

To answer these two questions we briefly review what the literature tells us about the influences of the IR laser field on the measurement process of the Wigner time delay. The time delay in the photoemission of an EWP extracted via the attosecond streaking technique can be distorted by the IR laser field due to: (i) the continuum-continuum coupling, that is commonly known as the CLC [72, 74], (ii) the bound-bound coupling and (iii) the polarization effects, i.e., the strong distortion of the bound electron cloud during the laser interaction [164].

Here we will address the influence of the bound-bound coupling induced by the IR laser field in case of the HOMO. Our assumption is based on the fact that the energy gap, $\Delta E_{10}$, between the $5 \sigma$ and the next upper bound orbital is $\Delta E_{10}=0.06807$ a.u., which is very close to the photon energy of the IR laser field $1.55 \mathrm{eV}$ ( $\omega_{0}=0.057$ a.u.).

We have calculated the populations of the first five bound states of our asymmetric molecule potential model while the IR laser pulse is acting on the HOMO. The intensity of the IR laser field is $2 \times 10^{12} \mathrm{~W} / \mathrm{cm}^{2}$ and Fig. 5.28 shows the populations of those firsts five states as a function of time. We find that the IR laser field induces a coherent superposition of the HOMO and the upper excited orbital ( $5^{\text {th }}$ state) during the interaction.

Furthermore, one would wonder if the transferred population to the upper excited state is given by one photon absorption of the IR laser field and how this transferred population can distort the stereo Wigner time delay measurements when the peak intensity of the IR laser field is changing.

We have calculated firstly the final population of the upper excited state with respect to the HOMO as a function of the peak intensity of the IR laser field. These simulations take into account the fact that the whole population is located in the HOMO at the beginning of the laser-molecule interaction. The logarithm of the excited population versus the peak intensity of the IR laser field in logarithmic scale is depicted in Fig. 5.29. We find out that behaviour of this curve is a straight line which has a slope of one. This means that the electron transition to the upper excited state is governed by one-photon 


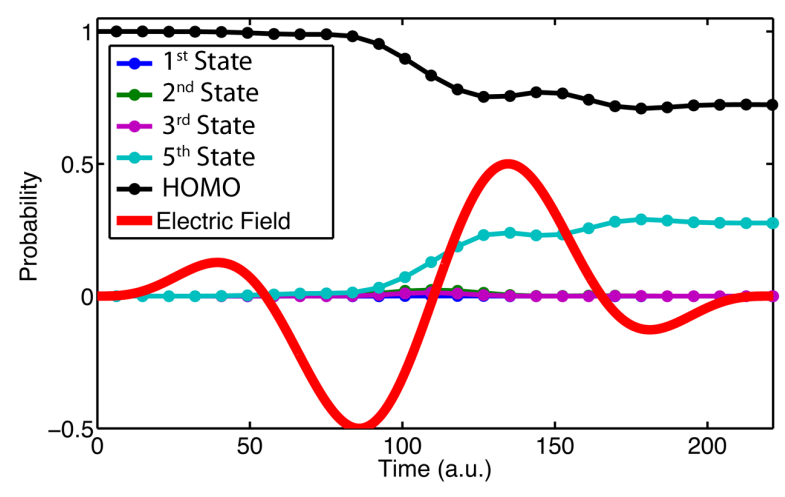

Figure 5.28: Coherent superposition induced by the IR laser field. The probability to find the electron in the first five bound states of our AM potential as a function of the time $t$ when the IR laser field acts on the $5 \sigma$ molecular orbital. The IR laser electric field $E_{\mathrm{L}}(t)$, is depicted in a red solid line. The laser parameters are the same as in Fig. 5.26.

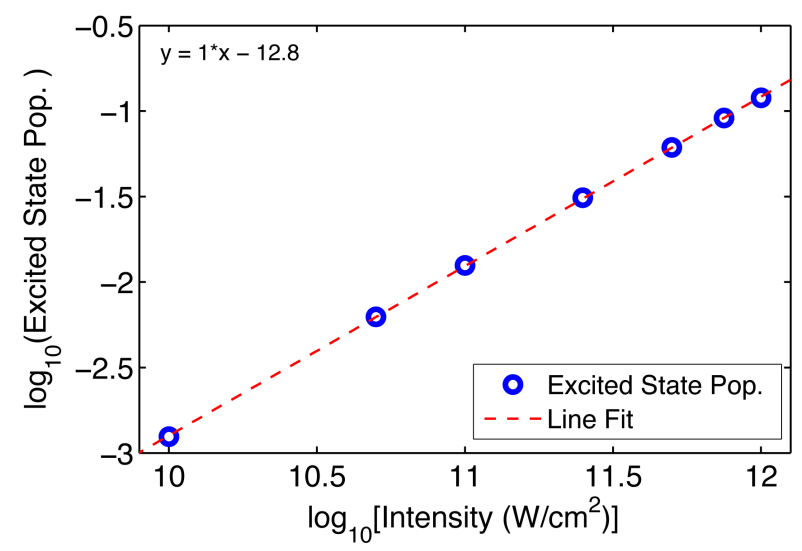

Figure 5.29: One photon absorption by an asymmetric molecular potential. Logarithm of the final population (blue circles) for the upper excited state with respect to the HOMO is shown as a function of he peak intensity of the IR laser field (in logarithmic scale). The excited population is scanned within a peak intensity range of $10^{10}-2 \times 10^{12} \mathrm{~W} / \mathrm{cm}^{2}$. The other parameters are the same as in Fig. 5.26. The red dashed line depicts a linear fit to the simulation data (note that the slope of this line is one). 
absorption from the IR laser field [162].

Secondly, we can expect that while the peak intensity of the IR laser field decreases the coherent transferred population decrease as well and therefore the stereo Wigner time delay can be recovered. However, we will find out that this is not the case. We have performed calculations of the stereo streaking time delays by fixing one photoelectron energy, i.e., the attosecond XUV frequency $\omega_{\mathrm{X}}=2.0$ a.u., and computing the streaking traces on the left-right as a function of the intensity of the IR laser.

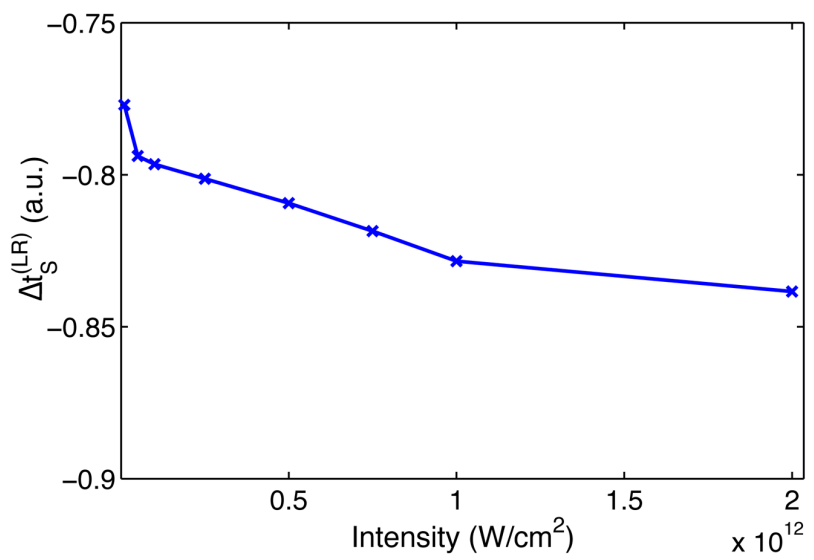

Figure 5.30: Stereo streaking time delay versus the peak intensity of the IR laser field. Stereo streaking time delay as a function of the peak intensity of the IR laser field for the HOMO. The attosecond XUV frequency is $\omega_{\mathrm{X}}=2.0$ a.u., its peak intensity $I_{\mathrm{X}}=5.0 \times 10^{12} \mathrm{~W} / \mathrm{cm}^{2}, \mathrm{FWHM}=9.48$ a.u. $(229 \mathrm{as})$, and $\mathrm{CEP}=\frac{\pi}{2}$ for all the intensities of the IR laser field.

Then, by the Fourier analysis of the left-right momentum expectation values, we have extracted the stereo streaking time delay. It is found that this delay does not exhibit an noticeable change. The results are depicted in Fig. 5.30. From this plot we verify that the stereo streaking time delay only changes about 1.5 as within the three orders of magnitude considered for the peak intensity of the IR laser field.

Although the transferred population to the excited state is about $10^{-3}$ at the smallest peak intensity of the IR laser field, the stereo streaking traces remain deformed and therefore the problems to extract the stereo Wigner time delay by our Fourier analysis.

We note that the stereo streaking traces are quite sensitive to the electron coherent superposition and this means the attosecond streaking camera can be a robust method to study such a bound electron dynamics process.

According to the $3 \mathrm{D}$ results we can conclude that it is problematic to measure the stereo time delay in case of the HOMO. The electron bound-bound transition dynamics 
induced by the IR laser suggests that when the XUV attosecond pulse ionizes this system, the left-right launched EWPs are coming from the coherent superposition of the HOMO and the upper excited state. Then, the left-right streaking traces are easily distorted and therefore the measuring of the stereo Wigner time delay.

It is important to mention that such a optical transition induced by the IR laser field is at one photon absorption.

On the other hand, the result for the stereo streaking time delay in case of the HOMO-1 shows that the stereo streaking technique works reasonably well to retrieve the stereo Wigner time delay when there is no optical transitions between bound states induced by the IR laser field.

In addition we point out that the asymmetry along the time delay in the stereo streaking traces might have rich information about the bound-bound electron dynamics induced by the IR laser field. As a final point next Section will be dedicated to explore the time delay asymmetry in the stereo streaking traces.

\subsubsection{Molecular bound-bound electron dynamics}

In the last Section we have shown that the attosecond streaking camera is not able to directly extract the stereo Wigner time delay in the case that the IR laser field induces a coherent superposition of two bound states in the medium.

In this Section we will take advantage of the attosecond streak camera and propose it to prove if the coherent superposition of those two bound states can be extracted. This temporary coherent superposition is mapped on the streaked EWPs. It is a clear sign of the encoded information about the bound-bound electronic dynamics.

Let us go back to the question related to the asymmetry on the momentum distribution along the time delay $\tau$-axis between the XUV and the IR laser for the stereo streaking traces. To this end, we define first the asymmetry $\operatorname{Asym}(\tau)$ :

$$
\operatorname{Asym}(\tau)=\frac{\mathrm{S}_{e}^{(\mathrm{L})}(\tau)-\mathrm{S}_{e}^{(\mathrm{R})}(\tau)}{\mathrm{S}_{e}^{(\mathrm{L})}(\tau)+\mathrm{S}_{e}^{(\mathrm{R})}(\tau)},
$$

where the quantity $\mathrm{S}_{\mathrm{e}}^{(\mathrm{L} / \mathrm{R})}(\tau)=\int_{0}^{\mp p_{\mathrm{zf}}} d p_{z} \mathrm{~S}_{\mathrm{e}}\left(p_{z}, \tau\right)$ is the ionized population emitted on the left-right by the XUV in presence of a weak IR laser field.

The calculated numerical results for the stereo streaking traces are depicted in Figs. 5.26a-b). We have computed the asymmetry $\operatorname{Asym}(\tau)$ for the ionized EWPs from the HOMO. In order to compare the $\operatorname{Asym}(\tau)$ of the HOMO to a system which does not 
a)

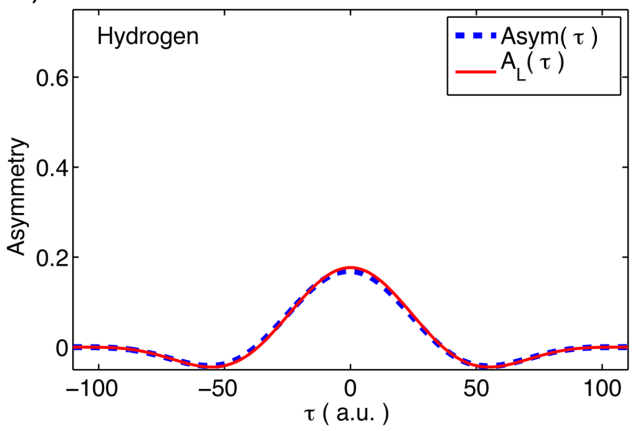

b)

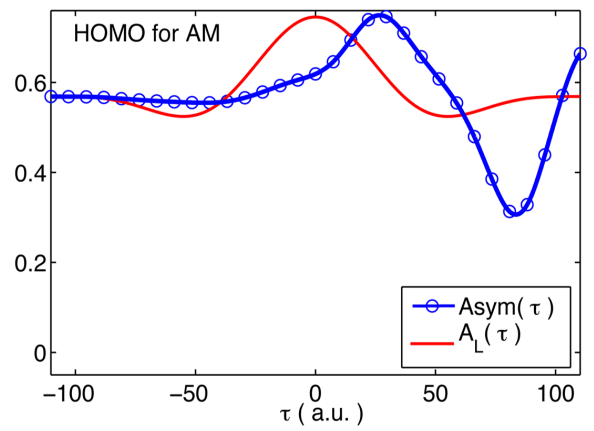

Figure 5.31: Time delay asymmetry on the streaking trace. a-b) Asymmetries Eq. (5.17), as a function of the time delay $\tau$ from the calculated stereo streaking traces from the ground state of an hydrogen atom (a) (blue dashed line) and the the HOMO of an AM (b) (blue line with circles) potential, respectively. The vector potential $A_{\mathrm{L}}(\tau)$ of the IR laser field is depicted in a red line. For both cases a) and b), the IR laser field parameters are the same as in Fig. 5.25 and the XUV attosecond pulse has a central frequency of 2.0 a.u., peak intensity is $I_{\mathrm{X}}=5 \times 10^{12} \mathrm{~W} / \mathrm{cm}^{2}$, its envelope is a gaussian with $\mathrm{FWHM}=250 \mathrm{as}$, and its $\mathrm{CEP}=\frac{\pi}{2}$.

allow the bound-bound transition by the IR laser field, the hydrogen atom is chosen. Then, we numerically computed the stereo streaking traces in the case of the ground state of $\mathrm{H}$ atom by the 3D TDSE. Thus, the asymmetry $\operatorname{Asym}(\tau)$ is calculated. The XUV and the IR laser fields used to solve TDSE for the $\mathrm{H}$ atom are the same as the ones used in case of the AM.

Fig. 5.31 shows the results of the asymmetry as a function of the time delay between the XUV and the weak IR laser field for each system. We find out, on the one hand, that the asymmetry in the case of an $\mathrm{H}$ atom, is initially zero and follows exactly the vector potential of the IR laser field. On the other hand, in case of the HOMO, there is a strong asymmetry in the amplitude and oscillations for the time delay $\tau<0$ compared to $\tau>0$. Note, that in case of the HOMO the initial asymmetry is different than zero, which is totally expected according to the natural asymmetry of the HOMO. However, the increment of the oscillation amplitude compared to the $\mathrm{H}$, is clear signature that the asymmetry is a powerful quantity to study the electron bound-bound transitions induced in a system.

These results point out that the bound-bound electron dynamics is responsible of a strong oscillation in the asymmetry in the time delay $\tau>0$, see Fig. 5.31b), and therefore the difficulties to extract the stereo Wigner time delay by utilizing attosecond streaking technique. 


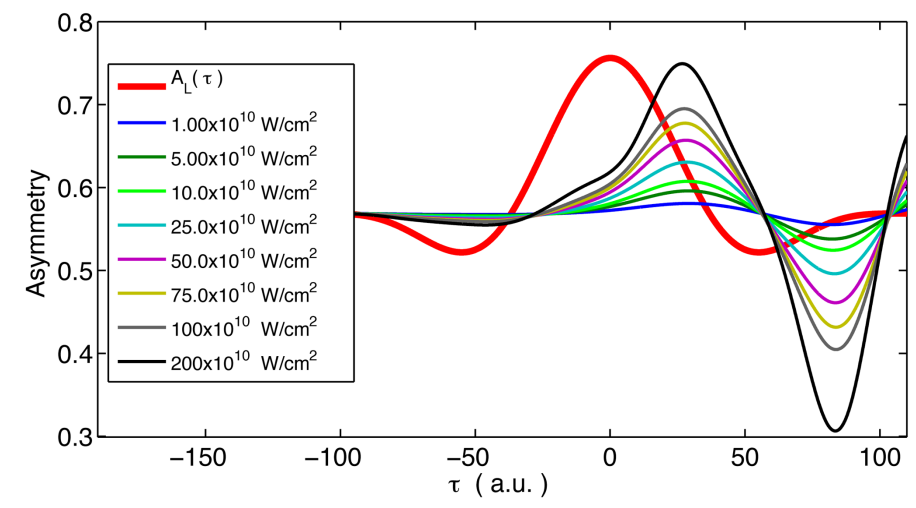

Figure 5.32: Asymmetry and scan on the peak intensity of the IR laser field. Asymmetries, as a function of the time delay and the peak intensity of the IR laser field, are depicted in different colors. The vector potential $A_{\mathrm{L}}(\tau)$ of the IR laser field is shown in a red line.

Now we address the natural question about the relation between the asymmetry and the transferred population to the upper excited state with respect to the HOMO. To this end, we test a broad range of asymmetries by using the attosecond streaking camera and changing the peak intensity of the IR laser field within $10^{10}-10^{12} \mathrm{~W} / \mathrm{cm}^{2}$.

Fig. 5.32 shows such asymmetry as a function of time delay and the peak intensity of the IR laser field. It is found out that the asymmetry as a function of the time delay changes by a constant amplitude factor when the intensity is increased.

In addition, we have computed the amplitude of the asymmetry $\operatorname{Asym}(\tau)$ and plotted such a quantity as a function of the peak intensity of the IR laser field. The results are depicted in Fig. 5.33a).

We find that the logarithm of the asymmetry amplitude increases linearly with the logarithm of the peak intensity and the slope of such a line is about 0.53. We also compare Fig. 5.33a) to b) which depicts the final excited population as a function of the peak intensity.

These results suggest that within the intensity range of our simulation the final transferred population to the upper excited electronic state can be measured by the asymmetric amplitude by utilizing the attosecond streaking technique.

Note that the asymmetry $\operatorname{Asym}(\tau)$ can be proposed as a suitable quantity to study the time evolution of the transferred population to upper excited states under the case of our molecular system. In other works, we find out that the "instantaneous" amplitude of the complex coefficient is related to the upper excited state and it could be mapped on the asymmetry at each time delay. This property points out that the 

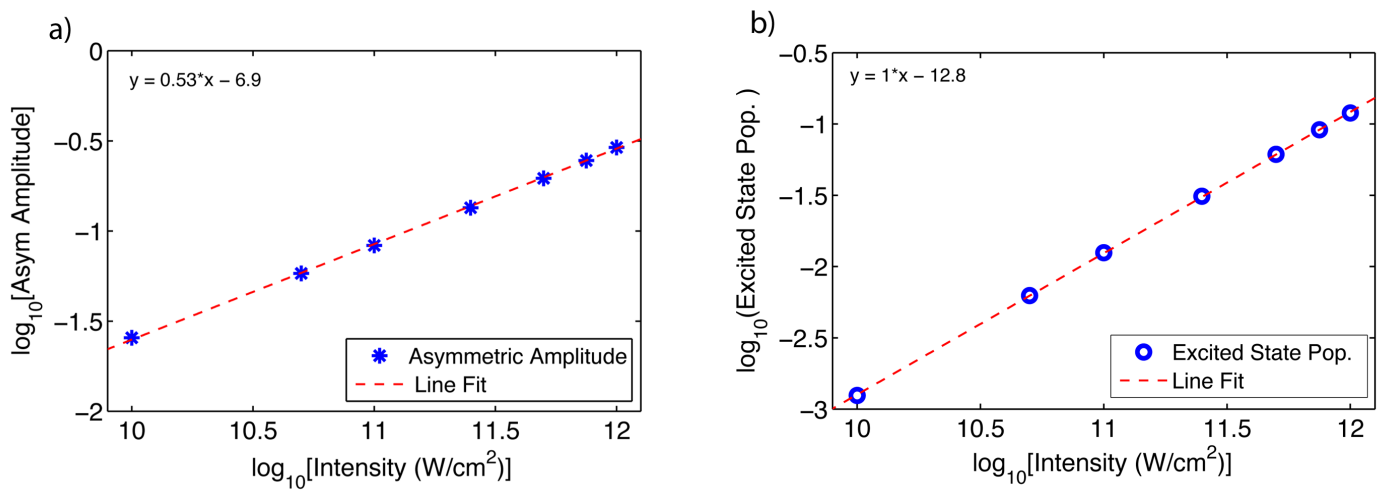

Figure 5.33: Asymmetry amplitude and excited population. a) Logarithm of the asymmetry amplitude (blue asterisk) form the stereo streaking trace of the HOMO as a function of the peak intensity of the IR laser field (in logarithmic scale). b) The logarithm of the final excited population transferred by the IR laser field from the HOMO to the upper excited state. The red dashed lines are a fit to a straight line.

attosecond streaking technique configures a very powerful tool to extract electronic bound dynamics information.

We finish this Section noting that the asymmetry also shows us that stereo streaking trace is strongly deformed by the induced coherent superposition of different states. This affects the measurement process of the stereo Wigner time delay when the optical transition is close to the one photon energy of the IR laser field.

We stress out that our results do not mean that the stereo streaking method does not work to retrieve the stereo time delay in the photoemission process. But the bound structure of the studied medium must be taken into account in the measurement process by the attosecond streak camera.

\subsection{Conclusions}

In this Chapter we have theoretically investigated the time delay in photoemission and its relation to the Wigner time delay.

In the first part, our numerical calculations show that EWP emitted by a single XUV attosecond pulse experiments a time delay in the process. This time delay depends strongly on the potential of the system. On the one hand, our 1D numerical results suggest that in case of short-range potentials the time delay in photoemission computed by utilizing the TOF method is exactly the Wigner time delay. On the other hand, in the case of long-range potentials the time delay in photoemission can not be directly 
related to the Wigner time delay. It is so because the strong influence the long-range behaviour of the potential has over the EWP, accelerating it during all its subsequent motion.

The attosecond streaking technique configures a useful tool to measure the time delay in photoemission depending on the atomic potential system. In the case of long-range potentials, the measurement of the time delay in photoemission by the streaking technique can not be directly related to the Wigner time delay. The coupling continuum-continuum between the IR laser field and the potential distorts the measurement process and induces an extra time delay which makes the streaking measurement difficult to correlate with the fundamental Wigner time delay.

Furthermore, in the second part, we propose an alternative way to extract information about the Wigner time delay. This approach is based on an asymmetric molecular system such as the CO and, by taking the left-right emitted EWPs, we define the stereo time delay in photoemission process as well as the stereo Wigner time delay. For the 1D molecular case, we demonstrate that this stereo TOF delay is exactly the stereo Wigner time delay and removes out the acceleration induced by the long-range nature of the Coulomb potential.

We point out that the measurement of the stereo streaking time delay by stereo streaking traces shows very good agreement to the stereo Wigner time delay. Then, we demonstrate that this scheme removes out the problematic CLC from the measurement process of the Wigner time delay.

Finally, in the third part of this Chapter we propose a 3D asymmetric potential model to confirm our 1D predictions about the stereo Wigner time delay. Our results suggest that the stereo Wigner time delay can also be measured in the case that there is no bound electron dynamics induced by the streaking field. In case where bound-bound electron dynamics are present, i.e., a coherent superposition induced by the IR laser field, the measurement of the stereo time delay in photoemission could be problematic by using the streaking technique.

In addition, we find out that the asymmetry as a function of the time delay between the XUV and IR laser field carry out information related to the molecular electron bound dynamics. We suggest that streaking technique might configure a very powerful tool in the study of the bound-bound transition electron dynamics on a system which has a electron energy gap between their states close to the photon energy of the IR laser field. 


\title{
Conclusions and outlook
}

\begin{abstract}
We organized the results of this thesis in two large groups. In the first group, the focus was on developing new techniques to characterize the complex electron wavefunction based on optical techniques. In the second group, the focus was on how to use the attosecond pulses to obtain dynamical information of the atomic and small molecular systems.
\end{abstract}

\section{Conclusions}

The main purpose of the first group of conclusions was regarding the study of different characterization techniques to reconstruct the amplitude and phase of an EWP and its corresponding complex bound-free dipole transition matrix element. In the second group of results the focus was on the study of the delay in photoemission process by the attosecond streaking technique, mainly a way to remove the so-called Coulomb-laser coupling.

\section{Electron wavepacket and structural information}

We have presented the QSPIDER technique in 1D and its 2D extension to reconstruct the complex EWP and its corresponding complex bound-free dipole transition matrix element.

Two different interferometric schemes were presented: the QSPIDER technique and the lateral momentum-shearing EWPs interferometry technique which is the extension of the QSPIDER to a 2D momentum plane.

With parameters that can be accessed today experimentally, we have shown that these techniques were able to recover structural information.

Thus, in Section 4.2 of Chapter 4, it was demonstrated that QSPIDER allows to extract the complex dipole within the EWP momentum-bandwidth. 


\section{CONCLUSIONS AND OUTLOOK}

In addition, the lateral momentum-shearing EWP interferometry technique was based on a similar to QSPIDER scheme, but in this case the streaked field was a circularly polarized one. According to our numerical results based on the SFA and 2D TDSE, we also demonstrated that this technique extracts structural information about the atomic target, i.e., the complex dipole.

We stress that the subsequent application of the two attosecond pulses in the presence of the IR laser field (linearly or circularly polarized) to the target demonstrated that QSPIDER is a single shot technique.

Furthermore, the emission of two EWPs from the same bound state showed that QSPIDER is a novel auto-referenced technique.

We concluded that in comparison to the high harmonic spectroscopy method $[5,65]$ used to extract structural information, QSPIDER has two desirable advantages: first, it is a single shot technique and second, an auto-referenced technique is provided.

Finally, the results of the Chapter 4 demonstrated that the concepts of the optical characterization techniques can be implemented successfully, at least under some conditions, for the electron wavepacket reconstruction.

\section{Wigner time delay and dynamical information}

The second group of results in this thesis was focused on the use of attosecond pulses to extract dynamical information of the ionization process. In particular, we investigated the problem of measuring the Wigner time in small asymmetric molecules.

The attosecond streaking technique is a useful tool to measure the delay in photoemission. Depending on the nature of the atomic potential this delay can be measured accurately.

For long-range potentials, the time delay in photoemission was not directly related to the Wigner time. The long-range contribution depended on the final position where the calculation was made and therefore the value was not unique. For short-range potential, the delay in photoemission and the Wigner time were the same.

In case of the short-range potential, the streaking technique worked very well to extract the Wigner delay. But for the long-range potentials, the coupling between the 
IR laser and the potential distorted the measurement and induced an extra delay.

In this thesis, we have proposed an alternative way to extract information about the Wigner delay. This method was based on an asymmetric molecular system such as CO. By taking the time delay difference between the emitted EWP on the left and right with respect to the molecular orientation axis, the stereo time delay in photoemission is defined. According to the described stereo delay in Section 5.3 of Chapter 5, our numerical results suggested that this stereo time delay in photoemission (stereo time of flight) was perfectly linked to the stereo Wigner delay. In addition, this asymmetric delay removed the acceleration induced by the long-range Coulomb tail.

We stress that the measurement of the stereo streaking time delay by two left-right streaking traces was compared in very good agreement to the stereo Wigner time delay. This viable scheme removed the problematic Coulomb-laser coupling from the measurement process of the Wigner time delay.

Furthermore, in Chapter 5, we explored a 3D asymmetric potential well model to confirm our 1D predictions regarding the stereo Wigner time delay. The results suggested that the stereo Wigner time delay can also be measured in case that there was no bound electron dynamics induced by the attosecond streak field. In case that there was bound-bound electron dynamics, i.e., a coherent superposition induced by the IR laser field, the measurement of the stereo delay in photoemission might be very hard. This was the case, if the gap energy between the coupled orbitals was about one photon of the IR laser.

\section{Outlook}

We have confirmed that attosecond science tools can be successfully applied to the electron structure and electron dynamics.

Our results suggest that the development of applications based on attosecond physics provided a valuable new perspective of electrons in the matter by accessing to the wavefunction itself.

As an outlook, we propose to extract structural information about the bound-free dipole transition matrix element on a broader momentum range than QSPIDER. By mixing the signal of a single XUV attosecond pulse and the ionization led by the IR laser 
field perhaps a full complex dipole reconstruction can be achieved. It would be very interesting to perform a full tomography reconstruction of the initial wavefunction of the electron with unprecedented spatial resolution. 


\section{Appendix A}

\section{A.1 Semiclassical aspects of the delay in photoemission}

While the Wigner definition can be used in a quantum context, it can also be linked to a classical description. To this end we assign a trajectory to the emitted EWP and from this we extract the delay in photoemission.

We will explain how the semiclassical trajectory is obtained from the injected EWP to the continuum by a XUV attosecond pulse. The extrapolation method to extract the information of the delay in photoemission is defined by considering this electron trajectory. We compare our calculated semiclassical electron trajectory to the corresponding analytical electron trajectory obtained via the stationary phase method.

We solve numerically the TDSE with Hamiltonian $H=\frac{1}{2}\left(p_{z}+A_{\mathrm{X}}(t)\right)^{2}+V_{0, \mathrm{Y} / \mathrm{C}}(z)$ to simulate the full electron wave function, $\Psi(z, t)$, of the systems. Here $p_{z}=-i \frac{\partial}{\partial z}$ denotes the momentum operator, $A_{\mathrm{X}}(t)=-\int^{t} E\left(t^{\prime}\right) d t^{\prime}$, is the vector potential of the XUV attosecond pulse with, $E_{\mathrm{X}}(t)$, its electric field and $V_{0, \mathrm{Y} / \mathrm{C}}(z)$, are the Yukawa and Coulomb potentials. The electric field of the XUV radiation is defined $E_{\mathrm{X}}(t)=$ $E_{0, \mathrm{X}}(t) \sin \left[\omega_{\mathrm{X}} t+\varphi_{0}\right]$. Where, $E_{0, \mathrm{X}}(t)$, denotes the envelope and $\varphi_{0}$, the carrier envelope phase of the pulse.

The continuum complex EWP, $\Psi_{c}(z, t)$, is computed by masking all the bound states from the whole wave function $\Psi(z, t)$ in case of $V_{0 \mathrm{Y}}$. That is, $\Psi_{c}(z, t)=f_{\text {Mask }}(z) \Psi(z, t)$, where, $f_{\text {Mask }}(z)$, is the mask function explained in the Section 2.2.1.7 of Chapter 2. In case of $V_{0, \text { C }}$ we calculate, $\Psi_{c}(z, t)$, by projecting out the first fours bound states of less energy.

The left, $\left\langle z_{\mathrm{L}}\right\rangle$ and right, $\left\langle z_{\mathrm{L}}\right\rangle$, position expectation values are calculated from the emitted EWP $\Psi_{c}(z, t)$ : 


$$
\begin{aligned}
& \left\langle z_{\mathrm{L}}\right\rangle=\frac{1}{\mathcal{N}_{\mathrm{L}}} \int_{-\infty}^{0} z\left|\Psi_{c}(z, t)\right|^{2} d z, \\
& \left\langle z_{\mathrm{R}}\right\rangle=\frac{1}{\mathcal{N}_{\mathrm{R}}} \int_{0}^{\infty} z\left|\Psi_{c}(z, t)\right|^{2} d z .
\end{aligned}
$$

Where $\mathcal{N}_{\mathrm{L}}=\int_{-\infty}^{0}\left|\Psi_{c}(z, t)\right|^{2} d z$ and $\mathcal{N}_{\mathrm{R}}=\int_{0}^{\infty}\left|\Psi_{c}(z, t)\right|^{2} d z$ are the ionized population to the left and right, respectively.

The EWP momentum distribution is calculated by projecting the continuum complex position EWP on plane and scattering waves $\Psi_{c}(k, t)=\int_{-\infty}^{\infty} \Psi_{k}^{*}(z) \Psi_{c}(z, t) d z$. By these momentum distributions, we can track in time the left and right momentum expectation values $\left\langle k_{\mathrm{L}}\right\rangle$ and $\left\langle k_{\mathrm{R}}\right\rangle$, respectively. These are defined by:

$$
\begin{aligned}
& \left\langle k_{\mathrm{L}}\right\rangle=\frac{1}{\mathcal{N}_{\mathrm{L}}^{(k)}} \int_{-\infty}^{0} k\left|\Psi_{c}(k, t)\right|^{2} d k, \\
& \left\langle k_{\mathrm{R}}\right\rangle=\frac{1}{\mathcal{N}_{\mathrm{R}}^{(k)}} \int_{0}^{\infty} k\left|\Psi_{c}(k, t)\right|^{2} d k,
\end{aligned}
$$

where $\mathcal{N}_{\mathrm{L}}^{(k)}=\int_{-\infty}^{0}\left|\Psi_{c}(k, t)\right|^{2} d k$ and $\mathcal{N}_{\mathrm{R}}^{(k)}=\int_{0}^{\infty}\left|\Psi_{c}(k, t)\right|^{2} d k$ are the ionized population on the left and right, respectively. These simples set of quantities the semiclassical electron trajectory: the position and momentum expectation values will allow us to recover the delay in photoemission for both potential systems.

\section{A.2 Extrapolation method}

Here we present the extrapolation method as a technique to extract the delay in photoemission.

We define the extrapolation method to extract the delay in photoemission or the Wigner time for the EWP, by tracking in time the semiclassical electron trajectory, $\langle z\rangle$, which is matched with the straight line $z_{\mathrm{M}}\left(t^{\prime}\right)$ :

$$
z_{\mathrm{M}}\left(t^{\prime}\right)=v(t)\left(t^{\prime}-\Delta t_{\mathrm{DIP}}\right)
$$

where $v(t)$ is the instantaneous electron velocity $v(t)=\frac{d\langle z\rangle}{d t}$. This velocity coincides with the EWP group velocity in short-range potential [157]. The quantity $\Delta t_{\text {DIP }}=$ $t_{i}-t_{a}$ is the delay in photoemission, where $t_{i}$ is the "ionization time" and $t_{a}$ is time at the max of the attosecond XUV pulse envelope. 


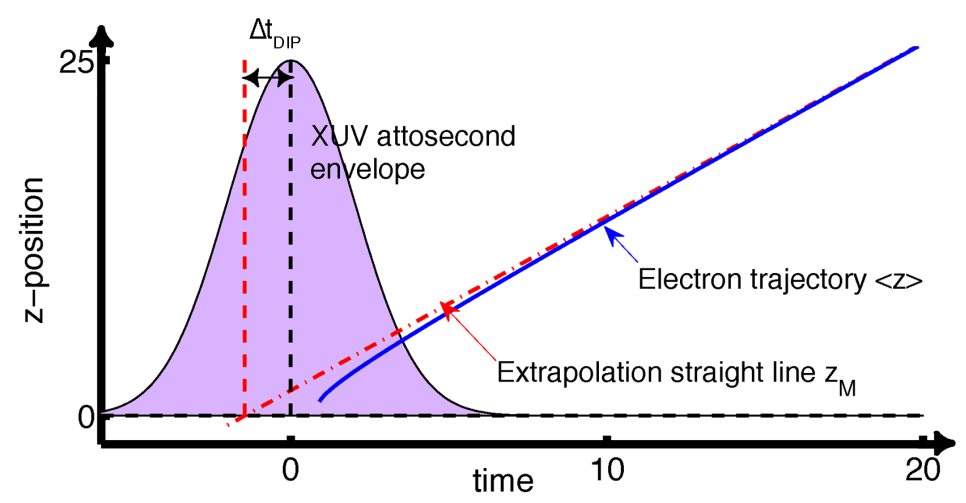

Figure A.1: Extrapolation method and the delay in photoemission. The blue line depicts the semiclassical electron trajectory $\langle z\rangle$ and the red dashed line with points shows the straight line trajectory $z_{\mathrm{M}}$, which is matched with the $\langle z\rangle$ at a certain time-position point $(t$, $z)$. The violet area is the attosecond pulse envelope and the black dashed vertical line indicates the max of the envelope. The delay in photoemission $\Delta t_{\text {DIP }}$ is the time duration between the "ionization time", $t_{i}$, (red dashed vertical line) and the time at the max of the attosecond pulse envelope, $t_{a}$, (back dashed vertical line).

By fixing the detection time-position point $(t, z)$ and the instantaneous velocity $v(t)$, the delay $\Delta t_{\text {DIP }}$ can be extracted via back extrapolation of the straight line $z_{\mathrm{M}}\left(t^{\prime}\right)$ to the $z=0[157,165]$. This procedure is illustrated in Fig. A.1.

We note that the delay in photoemission is based on the assumption that the electron appear at the zero position in continuum.

We will employ the extrapolation method to extract the associated delay in the photoemission for the short-range and long-range potentials.

In the next Section we describe the stationary phase method in order to obtain an analytical expression to the semiclassical electron trajectory. From this derivation, it is possible to compare our numerical calculation of the electron trajectory.

\section{A.3 Stationary phase method}

The time that an EWP takes to leave from its binding potential is related to the Wigner time. The ionization of an atomic system by a SAP is described by the complex electron wave packet $\Psi_{c}(z, t)$. After the attosecond pulse when the EWP has been formed, the complex wave packet is:

$$
\Psi_{c}(z, t)=\int a(k) \Psi_{k}(z) \exp [-i E t] d k
$$


where, $a(k)=|a(k)| \exp [i \phi(k)]$, is the complex ionization amplitude and $\Psi_{k}(z)$ is the scattering wave function of the continuum spectrum with momentum $k$ [75] and energy $E=\frac{k^{2}}{2}$. The term $\phi(k)$ is phase of the ionization complex amplitude $a(k)$. When the interaction of the scattered wave with the scattering center is defined by a short-range potential, then $\Psi_{k}(z)$ is asymptotically considered $(z \rightarrow \infty)$ a plane wave $\Psi_{k}(z) \approx \frac{1}{\sqrt{(2 \pi)}} e^{i k z}$. In the asymptotic limit when the EWP is far away from the scattering center, one may ask, around what value of $z$ the EWP is peaked at the arrival time $t$. In this limit, the most favorable contribution of the integral Eq. (A.4) will occur around $k_{0}$ where the amplitude $|a(k)|$ is peaked [155]. Then, this momentum coincides with a point where the phase in the integral Eq. (A.4) is stationary:

$$
z+\left.\frac{\partial \phi(k)}{\partial k}\right|_{k=k_{0}}-k_{0} t=0 .
$$

Here $\phi(k)$ is the phase-shift of the scattered wave in the short-range center or the phase of the bound-free dipole transition matrix element [75, 166].

One can ask, what time takes an electron propagating under the action of this short-range center to arrive the position $z$ with respect to the a free particle when the motion start at $z=0$ and $t=0$. From Eq. (A.5) we can derive this time delay:

$$
t(z)-\frac{z}{k_{0}}=\left.\frac{1}{k_{0}} \frac{\partial \phi_{l}}{\partial k}\right|_{k=k_{0}}
$$

where $t(z)$ is the time that an electron spends until the arrival position $z$ under the short-range potential action and $z / k_{0}$ is the time that spends a free particle of the same momentum $k_{0}$. Then, for a particle with momentum $k$ this time delay, $\Delta t_{\mathrm{W}}=t(z)-\frac{z}{k}$, is:

$$
\Delta t_{\mathrm{W}}=\frac{1}{k} \frac{\partial \phi(k)}{\partial k}=\frac{\partial \phi}{\partial E} .
$$

For brevity, we will refer to this energy derivative of the quantum phase, $\phi$, as the Wigner time delay $\Delta t_{\mathrm{W}}$. Note that the sign convention for, $\Delta t_{\mathrm{W}}$, is such that positive (negative) values correspond to delayed (advanced) emission.

When the potential, $V_{0}(z)$ is a long-range potential at large distances like the Coulomb potential $V_{0}(z)=-\frac{Z}{|z|}$, the situation is totally different. The wave function $\Psi_{k}(z)$ is not a plane wave and becomes the scattering Coulomb wave which contains a phase term that is logarithmic in $z$. As it is shown in [75] the stationary phase point is given by the solution of the equation: 


$$
z+\left[-\frac{Z}{k_{0}^{2}} \ln \left|2 k_{0} z\right|+\frac{Z}{k_{0}^{2}}+\frac{\partial \phi}{\partial k}\left(k_{0}\right)\right]-k_{0} t=0,
$$

where, $z$, describes the electron trajectory in the action long-range Coulomb potential. In analogy to Eq. (A.6) the time delay between the electron of momentum, $k$, traveling under the action of the Coulomb scattering center (with charge $Z$ ) and the free particle is:

$$
t(z)-\frac{z}{k}=\frac{Z}{k^{3}}(1-\ln |2 k z|)+\Delta t_{\mathrm{W}} .
$$

This time delay has a logarithmic dependency term in $z$ which is cannot be avoided. The logarithm term, $\Delta t_{\mathrm{L} .-\mathrm{R} .}=\frac{Z}{k^{3}}(1-\ln |2 k z|)$, is the long-range time delay contribution in case of Coulomb potentials. This logarithmic contribution is quite problematic to define a time delay in the photoionization process from an atomic system with the long-range Coulomb behaviour [68, 157].

We will evaluate this analytical trajectories Eqs. (A.5) and (A.8) by computing the momentum phase derivative, $\left.\frac{\partial \phi(k)}{\partial k}\right|_{k=k_{0}}$, at the asymptotic momentum, $k_{0}$, from our numeric results for the EWP ionized by an XUV attosecond pulse in case of the shortrange Yukawa potential and the long-range Coulomb potential well. We choose, $k_{0}$, as the value around the momentum at max of the momentum electron density [155]. We note that the, $k_{0}$, is close to the asymptotic $(t \rightarrow \infty)$ momentum expectation value $\langle k\rangle$.

\section{A.4 Photoionization via a single attosecond pulse}

In this Section, we show our TDSE numerical results for the photoionization and the semiclassical analysis by the extrapolation method in order to extract the delay in photoemission. The emitted EWPs are ionized from the ground states of the shortrange Yukawa and long-range Coulomb potentials. We use also the classical electron trajectory derived from the stationary phase Eqs. (A.5) and (A.8) method to compare to our numerical semiclassical trajectory by TDSE.

The TDSE numeric results for the one-photon ionization from ground state of Yukawa and Coulomb potentials are shown in Figs. A.2 and A.3, respectively. The XUV attosecond pulse has a central frequency of $\omega_{\mathrm{X}}=0.95$ a.u., a peak intensity $1.0 \times 10^{12} \mathrm{~W} / \mathrm{cm}^{2}, \mathrm{FWHM}=22.5$ a.u. $(544 \mathrm{as})$, and $\mathrm{CEP}=0 \mathrm{rad}$. The pulse has a gaussian envelope shape. The position grid parameters are the same that the utilized to compute the dipole structure. Fig. A.2a) shows the position expectation value for 
electrons ionized to the right, $\left\langle z_{\mathrm{R}}\right\rangle$, the electron trajectory, $z_{\mathrm{S} .-\mathrm{P} .}$, by stationary phase method and the straight line, $z_{\mathrm{M}}$, as a function of time $t$.
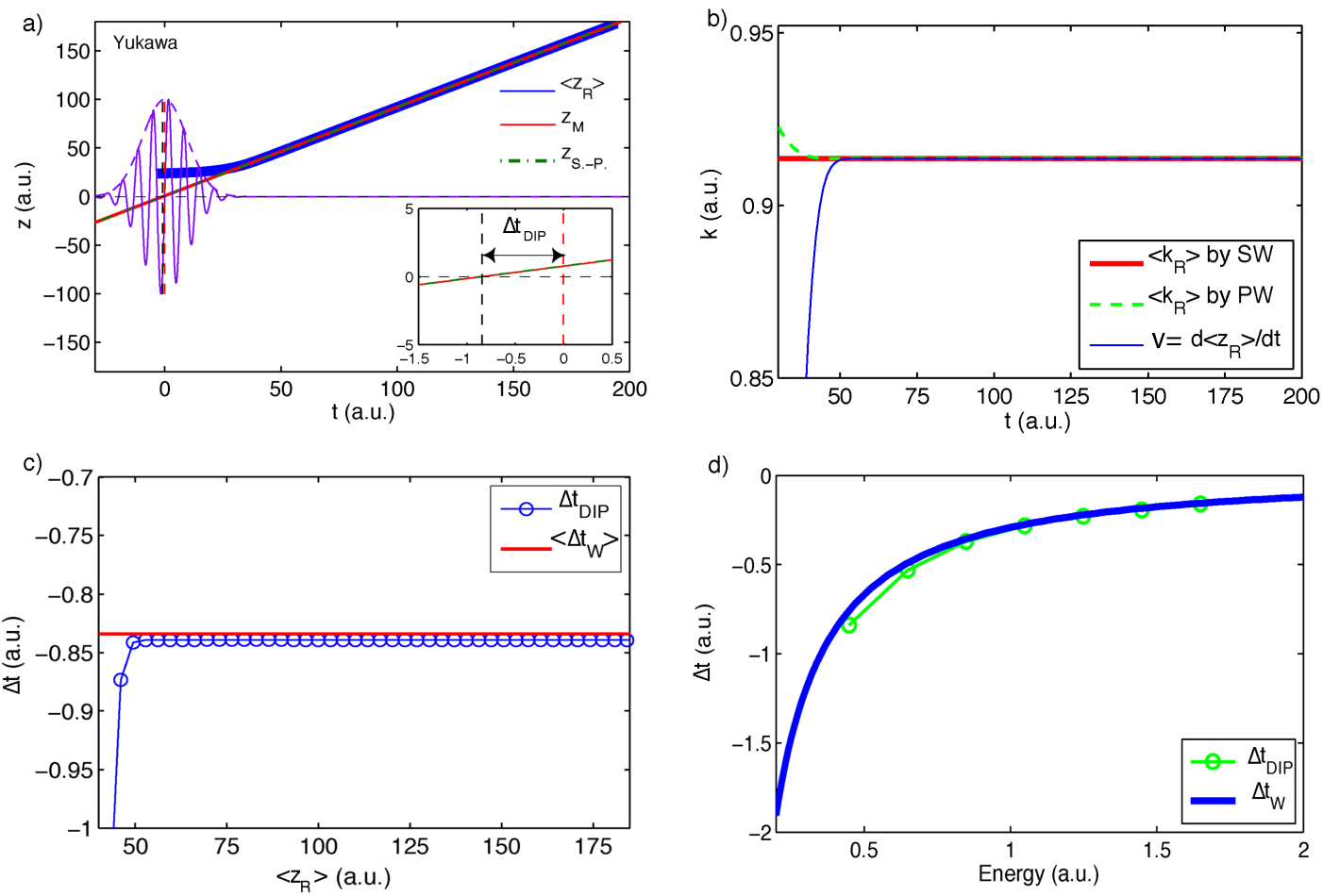

Figure A.2: Delay in photoemission from a short-range Yukawa potential. a) 1D TDSE calculations for the position expectation value $\left\langle z_{R}\right\rangle$ for an emitted EWP to the right (blue line), $z_{\mathrm{M}}$ (red line), the match to the straight line Eq. (A.3) and $z_{\mathrm{S} \text {.-P. the electron }}$ trajectory (green dashed line) under the stationary phase method. The attosecond pulse and its envelope are in violet line and violet dashed line, respectively. Inset graph shows the extrapolation of $z_{\mathrm{M}}$ to $z=0$ and how the delay $\Delta t_{\text {DIP }}$ with respect to $t=0$ (red dashed vertical line) is extracted. b) The expectation momentum value $\left\langle k_{\mathrm{R}}\right\rangle$ by scattering wave (SW red line), plane wave (PW green dashed line) and the time derivative (blue line) of the electron position $\left\langle z_{\mathrm{R}}\right\rangle$. c) The delay in photoemission (blue line and circles) as function of the position $\left\langle z_{\mathrm{R}}\right\rangle$ compared to the average of Wigner time $\left\langle\Delta t_{\mathrm{W}}\right\rangle$ (red line) within the energy bandwidth of the EWP. d) The time delay $\Delta t_{\text {DIP }}$ (green dotted line) for different EWPs of central energy $E_{0}=\omega_{\mathrm{X}}-I_{p}$, compared to exact Wigner time $\Delta t_{\mathrm{W}}(E)$ (blue line).

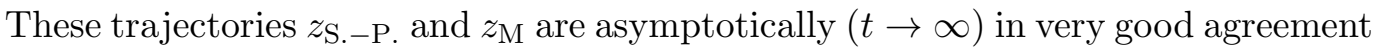
to the electron trajectory $\left\langle z_{\mathrm{R}}\right\rangle$ calculated by TDSE. The trajectory $z_{\mathrm{S} \text {.-P. has been }}$ computed by choosing the stationary momentum $k_{0} \approx 0.91$ a.u.

The straight line $z_{\mathrm{M}}(t)$ is computed by evaluating the instantaneous velocity $v\left(t_{d}\right)=$ $\left.\frac{d\left\langle z_{\mathrm{R}}\right\rangle}{d t}\right|_{t=t_{d}}=0.91$ a.u., at the time-position $\left(t_{d}=125, z_{d}=115\right)$ a.u. Then, the delay in photoemission $\Delta t_{\text {DIP }}$ is recovered by extrapolating $z_{\mathrm{M}}(t)$ back to $z=0$ (see inset graph 
in Fig. A.2a)). The delay gave a value about $\Delta t_{\text {DIP }}=-0.84$ a.u., for the EWP central energy $E_{0}=0.45$ a.u. Fig. A.2b) shows the momentum expectation values by plane and scattering wave projection as well as the time derivative of the position expectation value $\left(v\left(t_{d}\right)=\frac{d\left\langle z_{R}\right\rangle}{d t}\right)$.

The time derivative of the trajectory $\left\langle z_{\mathrm{R}}\right\rangle$ is compared to the momentum expectation values using SW and PW. These calculations show a very good convergence to the constant momentum $k_{0}=0.91$ a.u. by all the methods in case of the short-range Yukawa potential. The velocity variations shown for $t<50$ a.u., are due to the mask function $f_{\text {Mask }}(z)$ effects. This variation does not represent any physical meaning.

The delay in photoemission $\Delta t_{\mathrm{DIP}}$, extracted by the extrapolation method, as a function of the tracked electron position $\left\langle z_{\mathrm{R}}\right\rangle$ is depicted in Fig. A.2c). This delay $\Delta t_{\text {DIP }}$ converges to the value of -0.839 a.u. This is in very good agreement with the average Wigner time $\left\langle\Delta t_{\mathrm{W}}\right\rangle=-0.834$ a.u.

Here we define the average in the Wigner time:

$$
\left\langle\Delta t_{\mathrm{W}}\right\rangle=\frac{\int d E \Delta t_{\mathrm{W}}(E)\left|\Psi_{c}(E)\right|^{2}}{\int d E\left|\Psi_{c}(E)\right|^{2}},
$$

where, $\left|\Psi_{c}(E)\right|^{2}$, is the energy spectrum of the ionized EWP and $\Delta t_{\mathrm{W}}(E)$ the Wigner time delay.

The delay $\Delta t_{\mathrm{DIP}}$, for a broader range of EWP energies $E_{0}=\omega_{\mathrm{X}}-I_{p}$, is shown in Fig. A.2d) and compared to the exact Wigner time delay. To extract the delay $\Delta t_{\text {DIP }}$, we compute the different trajectories $z_{\mathrm{M}}$ for all the EWPs. By calculating the instantaneous velocity, $v(t)$, at the fixed position, $\left\langle z_{\mathrm{R}}\right\rangle=150$, the electron trajectories, $z_{\mathrm{M}}$, are obtained and from them the delay in photoemission, $\Delta t_{\mathrm{DIP}}$, is recovered for the different EWPs. The XUV attosecond frequencies, $\omega_{\mathrm{X}}$, are within the range of 0.95-2.15 a.u., the attosecond FWHM, the peak intensity and the CEP are the same that in the example shown in Fig. A.2a).

We find that the delay in photoemission $\Delta t_{\text {DIP }}$ is in very good agreement to the exact Wigner time in case of Yukawa short-range potential. We note that for low photoelectron energy $\left(E_{0}=0.45\right.$ a.u.) there is a small different between the exact Wigner time and the extracted delay in photoemission by the extrapolation method. This is due to the EWP takes the average Wigner time, $\left\langle\Delta t_{\mathrm{W}}\right\rangle$, within its energy bandwidth [165]. These numerical calculations show that the Wigner time delay can be extracted by the extrapolation method in case of short-range potentials. However, 
in case of hydrogen this delay in photoemission recovered by the present method is not the same that Wigner time.
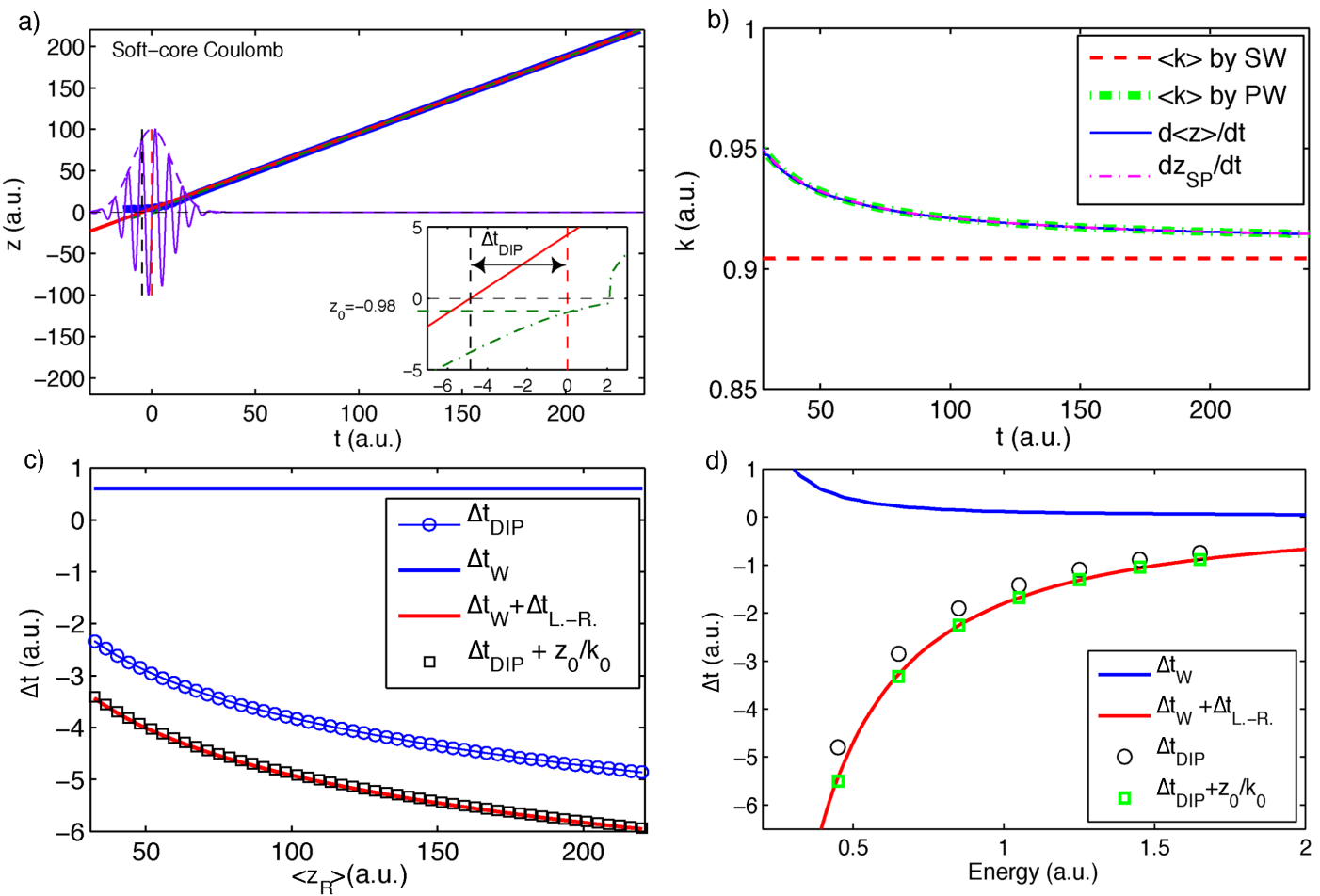

Figure A.3: Delay in photoemission from a long-range Coulomb potential. a) Same as in Fig. A.2a), but for the H Coulomb potential. Inset graph shows a zoom around the coordinate origin and the difference between the straight line, $z_{\mathrm{M}}$ and the trajectory, $z_{\mathrm{S} . \mathrm{P} . \mathrm{b}} \mathrm{b}$ ) The momentum expectation values, $\left\langle k_{\mathrm{R}}\right\rangle$, by SWs (red dashed line), PWs (green dashed line with points), instantaneous velocity $\frac{d\left\langle z_{\mathrm{R}}\right\rangle}{d t}$ (blue line) and $\frac{d z_{\mathrm{s} .-\mathrm{P}}}{d t}$ (magenta line). c) The delay in photoemission, $\Delta t_{\text {DIP }}$ (blue line and circles), average Wigner time (blue line), total time delay, $\Delta t_{\mathrm{Tot}}=\Delta t_{\mathrm{W}}+\Delta t_{\mathrm{L} . \mathrm{R} \text {. }}$ (red line) and the $\Delta t_{\mathrm{DIP}}$ with respect to the initial position $z_{0}=-0.98$ (black squares) as a function of the position, $\left\langle z_{\mathrm{R}}\right\rangle$, (in case of a single emitted EWP). d) The Wigner time delay $\Delta t_{\mathrm{W}}$ (blue line), the total time delay, $\Delta t_{\mathrm{W}}+\Delta t_{\mathrm{L} .-\mathrm{R} .}$, (red line), the delay in photoemission $\Delta t_{\text {DIP }}$ (black circles) by the extrapolation method with respect to $z_{0}=0$, and $\Delta t_{\text {DIP }}$ with respect to $z_{0}$ (green squares) as a function of the photo-electron energy $E_{0}$.

The Fig. A.3a) shows similar calculations that in Fig. A.2a) but for an EWP ionized from the ground state of the $\mathrm{H}$ long-range Coulomb potential. The attosecond pulse parameters are the same that in Yukawa potential. In spite of the semiclassical electron trajectory $\left\langle z_{\mathrm{R}}\right\rangle$ in Fig. A.3a) apparently follows a asymptotic straight line, its behavior is logarithmic. According to Eq. (A.8), this logarithmic behabiour is expected. This is a direct consequence of the long-range potentials, i.e., the Coulomb one $[75,68,165,156]$. 
To calculate the electron trajectory $z_{\text {S.P. }}$ from the stationary phase method, the stationary momentum $k_{0}$ and $z_{0}^{\prime}=-\left(\frac{1}{k_{0}^{2}}+\left.\frac{\partial \phi}{\partial k}\right|_{k=k_{0}}\right)$ require to be computed. The values of the stationary momentum is $k_{0} \approx 0.91 \mathrm{a} . \mathrm{u}$.

The trajectory, $z_{\text {S.P. }}$, is in very good agreement to the numerical one, $\left\langle z_{\mathrm{R}}\right\rangle$, in the asymptotic limit $(t \rightarrow \infty$ or $z \rightarrow \infty)$. For the out-going EWP propagation throughout the Coulomb potential, the instantaneous velocity, $v(t)=\frac{d\left\langle z_{\mathrm{R}}\right\rangle}{d t}$, is not constant any more (see Fig. A.3b)) in the asymptotic limit.

The electron velocity $v(t)$ is time-position dependent and this implicates that the delay in photoemission extracted by the extrapolation method will depend of the detection time-position $\left(t,\left\langle z_{\mathrm{R}}\right\rangle\right)$. Fig. A.3b) shows this velocity $v(t)$ as a function of time $t$ compared to the momentum expectation values by scattering wave and plane wave as well as the time derivative $v_{\mathrm{S} .-\mathrm{P}}(t)=\frac{d z_{\mathrm{S} .-\mathrm{P}}}{d t}$.

The electron velocities $v_{\mathrm{S} .-\mathrm{P}}(t), v(t)$ and the momentum expectation values, $\left\langle k_{\mathrm{R}}\right\rangle$ by the PWs, are asymptotically the same. Their time dependency shows that the electron is accelerated when it is propagating throughout the long-range tail of the Coulomb potential well.

We note that the scattering waves yield a constant asymptotic momentum, $\left\langle k_{\mathrm{R}}\right\rangle$, immediately after the end of attosecond pulse while this is no the case for momentum expectation value calculated by plane waves. This indicates that the plane wave describes the kinetic momentum related to the ionized EWP which is propagating throughout the Coulomb potential. In addition, if the analytical electron trajectory, $z_{\mathrm{S} .-\mathrm{P}}$, is known the stationary momentum, $k_{0}$, (which is close to $\left\langle k_{R}\right\rangle$ ) can be extracted by fitting, $\frac{d z_{\mathrm{S} .-\mathrm{P}}}{d t}$, to momentum expectation value, $\left\langle k_{R}\right\rangle$, by the plane wave method. It is important in case that scattering wave shall be unknown.

The instantaneous velocity is computed at the time-position $\left(t_{d}=236,\left\langle z_{\mathrm{R}}\right\rangle=220\right)$ a.u., and has a value of $v\left(t_{d}\right)=0.9145$ a.u. Using these quantities the straight line $z_{\mathrm{M}}$ is constructed. The time delay in photoemission extracted by the extrapolation method is $\Delta t_{\text {DIP }}=-4.86$ a.u. Insets graph in Fig. A.3a) shows a detail information related to the extraction of the delay in photoemission.

We have tracked this delay, $\Delta t_{\mathrm{DIP}}$, as a function of the position $\left\langle z_{\mathrm{R}}\right\rangle$ between 32 and 220 a.u. (faraway from the core). The results are depicted in Fig. A.3c). We find that this time delay $\Delta t_{\text {DIP }}$ depends strongly on the electron position, $\left\langle z_{\mathrm{R}}\right\rangle$, and has a logarithmic behaviour.

This means that there is no way to demonstrate that in case of long-range potential the delay in photoemission is well defined in the sense that this delay has a good asymptotic convergence limit as a function of the position $\left\langle z_{\mathrm{R}}\right\rangle$. The contribution of long-range 
delay is computed by evaluating $\Delta t_{\mathrm{L} .-\mathrm{R} .}\left(z_{\mathrm{R}}\right)$ as a function of the electron position $\left\langle z_{\mathrm{R}}\right\rangle$ and fixing the momentum to $k_{0}$. The total delay $\Delta t_{\text {Tot }}\left(z_{\mathrm{R}}\right)=\left\langle\Delta t_{\mathrm{W}}\right\rangle+\Delta t_{\mathrm{L} .-\mathrm{R} .}\left(z_{\mathrm{R}}\right)$ is compared to the delay in photoemission $\Delta t_{\text {DIP }}$ and we have again found that there is no good agreement between the total delay and $\Delta t_{\text {DIP }}$ (see Fig. A.3c)).

Furthermore, we note that this discrepancy can be solved if the position $z=0$ (point with respect to the time delay $\Delta t_{\text {DIP }}$ is extracted) is changed by $z=z_{0}$. As it is depicted in inset Fig. A.3a) the delay in photoemission $\Delta t_{\text {DIP }}$ can be detected from the position $z_{0}=-0.98$. This position is no arbitrary. It is related to the instantaneous

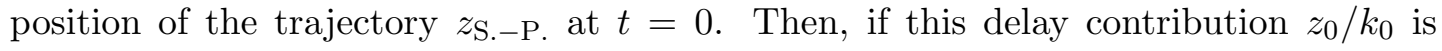
added to $\Delta t_{\text {DIP }}$ we find a perfect agreement between the exact total delay $\Delta t_{\text {Tot }}$ and the new referenced delay in photoemission $\Delta t_{\text {DIP }}+z_{0} / k_{0}$.

The delay in photoemission $\Delta t_{\text {DIP }}$ as a function of the photo-electron central energy $E_{0}$ is shown in Fig. A.3d). To extract the time delay $\Delta t_{\text {DIP }}$ by the extrapolation method, we fix the same arrival position $\left\langle z_{\mathrm{R}}\right\rangle=216$ a.u.

The comparison between the delay $\Delta t_{\mathrm{DIP}}$, the time delay $\Delta t_{\mathrm{W}}$ and the total time delay $\Delta t_{\text {Tot }}$ show that there is a disagreement between these quantities. However, a prefect agreement is found between the delay in photoemission and the total delay when $\Delta t_{\text {DIP }}$ is measured with respect to a certain position $z_{0}$ (see Fig. A.3d)). This position $z_{0}$ is calculated for each EWP (with stationary momentum $k_{0}$ ) by taking the value of

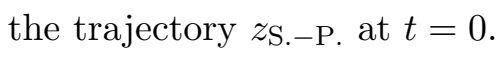

Finally, this semiclassical analysis of the momentum expectation value, $\left\langle k_{\mathrm{R}}\right\rangle$, and the semiclassical velocity, $v=\frac{d\left\langle z_{\mathrm{R}}\right\rangle}{d t}$, shows that the calculation of $\left\langle k_{\mathrm{R}}\right\rangle$ by the plane wave describes the kinetic momentum of the electron, whereas the calculation of $\left\langle k_{\mathrm{R}}\right\rangle$ by scattering wave gives the asymptotic momentum which takes into account the kinetic momentum and the acceleration of the binding potential.

Note this analysis shows us that the Wigner time delay in a long-range potential wells is difficult to extract by extrapolation method or a simple classical picture. In addition, any well-defined delay in photoemission can be assigned in the sense that there is no convergence limit in $\Delta t_{\text {DIP }}$ for long-range Coulomb potentials. However, in case of short-range potential system our results show that the delay in photoemission coincides in perfect agreement with the Wigner time. 


\section{Bibliography}

[1] A. Zewail. J. Phys. Chem. A, 104:5660, (2000). 1

[2] Y. Bhattacharjee. Nature, 412:474, (2001). 1

[3] F. Krausz and M. Ivanov. Rev. Mod. Phys., 81:163, (2009). 1, 2, 21, 26, 31, 83, $88,99,153$

[4] T. Zuo, A. D. Bandrauk, and P. B. Corkum. Chem. Phys. Lett., 259:313, (1996). 1

[5] J. Itatani, J. Levesque, D. Zeidler, et al. Nature, 432:867, (2004). 1, 13, 83, 198

[6] P. B. Corkum and F. Krausz. Nat. Phys., 3:381, (2007). 1, 3, 4, 5, 13, 26, 99

[7] E. Goulielmakis, M. Schultze, M. Hofstetter, et al. Science, 320:1614, (2008). 2

[8] T. H. Maiman. Nature, 187:493, (1960). 3

[9] U. Keller. Nature, 424:831, (2003). 3

[10] F. J. McClung and R. W. Hellwarth. J. Appl. Phys., 33:828, (1962). 3

[11] R. Paschotta. RP Photonics: Encyclopedia. http://www.rp-photonics.com/. 3

[12] L. E. Hargrove, R. L. Fork, and M. A. Pollack. Appl. Phys. Lett., 5:4, (1964). 3

[13] W. E. Lamb. Phys. Rev., 134:A1429, (1964). 3

[14] R. Borrego. Role of wavefront aberrations of amplified femtosecond pulses in nonlinear optics, 2012. PhD. thesis, Universidad de Salamanca. 3, 125

[15] P. F. Moulton. J. Opt. Soc. Am. B., 3:125, (1986). 3 


\section{BIBLIOGRAPHY}

[16] R. L. Fork, C. H. Brito Cruz, P. C. Becket, and C. V. Shank. Opt. Lett., 12:483, (1987). 4

[17] M. Schultze, M. Fiess, N. Karpowicz, et al. Science, 328:1658, (2010). 4, 14, 83, $86,145,147,154,161$

[18] K. Klünder, J. M. Dahlström, M. Gisselbrecht, et al. Phys. Rev. Lett., 106:143002, (2011). 4, 15, 83, 145

[19] D. Strickland and G. Mourou. Opt. Comm., 56:219, (1985). 3

[20] C. Iaconis and I. A. Walmsley. Opt. Lett., 23:792, (1998). 5, 84, 86, 105

[21] M. E. Anderson, L. E. E. Araujo, E. M. Kosik, and I. A. Walmsley. Appl. Phys.

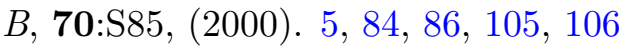

[22] E. M. Kosik, A. S. Radunsky, I. A. Walmsley, and Dorrer. C. Opt. Lett., 30:326, (2005). 5

[23] D. J. Kane and R. Trebino. Opt. Lett, 18:823, (1993). 5

[24] P. OShea, M. Kimmel, X. Gu, and R. Trebino. Opt. Lett., 26:932, (2001). 5

[25] B. Alonso, I. J. Sola, O. Varela, J. Hernández-Toro, et al. J. Opt. Soc. Am. B, 27:933, (2010). 5

[26] B. Alonso. Spatiotemporal characterization of ultrashort laser pulses, 2012. $\mathrm{PhD}$. thesis, Universidad de Salamanca. 5

[27] C. P. Hauri, W. Kornelis, F. W. Helbing, et al. Appl. Phys. B, 79:673, (2004). 5

[28] B. Varela, O. Alonso, I. J. Sola, J. San Román, et al. Opt. Lett., 35:3649, (2010). 5

[29] W. Holgado, B. Alonso, J. San Román, and I. J. Sola. Opt. Exp., 22:10191, (2014). 5

[30] F. Böhle, M. Kretschmar, A. Jullien, et al. Laser Phys. Lett., 11:095401, (2014). 5

[31] U. Keller, G. W. tHooft, W. H. Knox, and J. E. Cunningham. Opt. Lett., 16:1022, (1991). 5 
[32] T. R. Schibli, O. Kuzucu, J.-W. Kim, et al. J. Sel. Topics in Quantum Elec., 9:1022, (2003). 5

[33] M. Uiberacker, Th. Uphues, M. Schultze, et al. Nature, 446:627, (2007). 5

[34] A. L'Huillier and Ph. Balcou. Phys. Rev. Lett., 70:774, (1993). 5

[35] G. Mainfray and C. Manus. Rep. Prog. Phys., 54:1333, (1991). 5

[36] B. Walker, B. Sheehy, L. F. DiMauro, et al. Phys. Rev. Lett., 73:1228, (1994). 5

[37] M. Hentschel, R. Kienberger, Ch. Spielmann, et al. Nature, 414:509, (2001). 5, $6,10,12,14,145$

[38] P. M. Paul, E. S. Toma, P. Breger, et al. Science, 292:1689, (2001). 5, 6, 10, 11, $12,15,145$

[39] M. Drescher, M. Hentschel, R. Kienberger, et al. Nature, 419:803, (2002). 6

[40] A. McPherson, G. Gibson, H. Jara, et al. J. Opt. Soc. Am. B, 4:595, (1987). 6

[41] M. Ferray, A. LHuillier, X. F. Li, G. Mainfray, and C. Manus. J. Phys. B, 21:L31, (1988). 6

[42] P. B. Corkum. Phys. Rev. Lett., 71:1994, (1993). 6

[43] M. Lewenstein, Ph. Balcou, M. Y. Ivanov, A. L'Hullier, and P. B. Corkum. Phys. Rev. A, 49:2117, (1994). 7, 19, 90

[44] Y. Mairesse, A. de Bohan, D. Bauer, L. J. Frasinski, et al. Science, 302:1540, (2003). 7, 89

[45] A.-T. Le, T. Morishita, and C. D. Lin. Phys. Rev. A, 78:023814, (2008). 7

[46] A.-T. Le, R Della Picca, P. D. Fainstein, et al. J. Phys. B, 41:081002, (2008). 7

[47] M. Y. Ivanov, T. Brabec, and N. Burnett. Phys. Rev. A, 54:742, (1996). 7

[48] J. Z. Kaminski and F. Ehlotzky. Phys. Rev. A, 54:3678, (1996). 7

[49] M. F. Ciappina, C. C. Chirila, and M. Lein. Phys. Rev. A, 75:043405, (2007). 7

[50] P. Mulser and D. Bauer. High Power Laser-Matter Interaction. Springer Verlag Berlin Heidelberg, 2010. 9, 22, 23, 26, 29, 56 


\section{BIBLIOGRAPHY}

[51] G. Farkas and C. Toth. Phys. Lett. A, 168:447, (1992). 10

[52] P. B. Corkum, N. H. Burnett, and M. Y. Ivanov. Opt. Lett. A, 19:1870, (1994). 10

[53] I. J. Sola, E. Mével, L. Elouga, E. Constant, et al. Nature Phys., 2:319, (2006). 10,11

[54] G. Sansone, E. Benedetti, F. Calegari, et al. Science, 314:443, (2006). 10

[55] F. He, C. Ruiz, and A. Becker. Opt. Lett., 32:3224, (2007). 10

[56] C. Hernández García. Coherent attosecond light sources based on high-order harmonic generation: influence of the propagation effects, 2013. PhD. thesis, Universidad de Salamanca. 10

[57] H. G. Muller. Appl. Phys. B, 74:S17, (2002). 11

[58] Y. Mairesse and F. Quéré. Phys. Rev. A, 71:011401(R), (2005). 12, 83, 84, 96, 99, 107

[59] J. Itatani, F. Quéré, G. L. Yudin, et al. Phys. Rev. Lett., 88:173903, (2002). 12, 96,99

[60] M. Kitzler, N. Milosevic, A. Scrinzi, F. Krausz, and T. Brabec. Phys. Rev. Lett., 88:361, (2002). 12

[61] F. Quéré, Y. Mairesse, and J. Itatani. J. Mod. Opt., 52:339, (2005). 12, 14, 83, 84,145

[62] F. Quéré, J. Itatani, G. L. Yudin, et al. Phys. Rev. Lett., 90, (2003). 12, 83, 84, 99, 104, 107, 145

[63] P. Eckle, M. Smolarski, P. Schlup, et al. Nature Phys., 4:565, (2008). 13

[64] F. krausz and M. I. Stockman. Nature Photonics, 8:205, (2014). 13, 31

[65] S. Haessler, J. Caillat, W. Boutu, et al. Nature Phys., 6:200, (2010). 13, 83, 198

[66] T. Remetter, P. Johnsson, J. Mauritsson, et al. Nature Phys., 2:323, (2006). 14, $83,84,86$

[67] A. Chacón, M. Lein, and C. Ruiz. Phys. Rev. A, 87:023408, (2013). 14, 84, 88, 145 
[68] J. M. Dahlström, A. L'Huillier, and A. Maquet. J. Phys. B, 44:081001, (2011). 14, 15, 145, 148, 152, 153, 159, 161, 205, 208

[69] E. P. Wigner. Phys. Rev., 98:145, (1955). 14, 145, 147, 148, 152

[70] F. T. Smith. Phys. Rev., 108:349, (1960). 14, 145

[71] L Eisenbud. The formal properties of nuclear collisions, 1948. PhD. thesis, Princeton University. 14, 145, 147

[72] S. Nagele, R. Pazourek, J. Feist, et al. J. Phys. B: At. Mol. Opt. Phys., 44:081001, (2011). 15, 147, 151, 152, 154, 159, 171, 189

[73] A. S. Kheifets and I. A. Ivanov. Phys. Rev. Lett., 105:233002, (2010). 15, 159

[74] M. Ivanov and O. Smirnova. Phys. Rev. Lett., 107:213605, (2011). 15, 159, 160, 171,189

[75] V. Serov, V. L. Derbov, and T. A. Sergeeva. Phys. Rev. A, 87:063414, (2013). $15,146,148,152,161,204,208$

[76] Q.-C. Ning, L.-Y. Peng, S.-N. Song, et al. Phys. Rev. A, 90:013423, (2014). 15, 146

[77] A. Chacón, M. Lein, and C. Ruiz. Phys. Rev. A, 89:053427, (2014). 15, 165

[78] L. V. Keldysh. Eksp. Teor. Fiz., 47:1945, (1964). 19, 29, 30

[79] F. H. M. Faisal. J. Phys. B., 6:L89, (1973). 19, 29, 30

[80] H. R. Reiss. Phys. Rev. A, 22:1786, (1980). 19, 29, 30

[81] A. Becker and F. H. Faisal. J. Phys. B, 38:R1, (2005). 19

[82] H. Hertz. Annalen der Physik, 267:983, (1887). 19

[83] A. Peralta Conde, L. P. Yatsenko, J. Klein, et al. Phys. Rev. A, 72:053808, (2005). 19

[84] A. Peralta Conde, J. Kruse, O. Faucher, et al. Phys. Rev. A, 79:061405R, (2009). 19

[85] A. Becker and F. H. M. Faisal. J. Phys. B, 38:R1, (2005). 19 


\section{BIBLIOGRAPHY}

[86] J. J. Sakurai. Modern Quantum Mechanics. Addison Wesley, 1994. 22, 23, 24, $25,27,28,37,88$

[87] L. Plaja. Teoría Perturbativa de la Ionización , (2007). Notas de Máster. 22

[88] J. R. Ackerhalt and P. W. Milonni. J. Opt. Soc. Am. B, 1:116, (1984). 23

[89] T. K. Kjeldsen. Wave packet dynamics studied by ab initio methods: Applications to strong-field ionization of atoms and molecules, 2007. PhD. thesis, University of Aarhus. 24, 34, 37, 38, 39

[90] T. Brabec. Strong Field Laser Physics. Springer Science, 2008. 26

[91] W. Gordon. Z. Phys., 40:294, (1926). 27

[92] D. V. Volkov. Z. Phys., 94:294, (1935). 27

[93] A. Lohr, M. Kleber, R. Kopold, and W. Becker. Phys. Rev. A, 55:R4003, (1997). 28,29

[94] D. B. Milošević, G. G. Paulus, D. Bauer, et al. J. Phys. B., 39:R203, (2006). 30, 43,56

[95] A. Persson. Numerical methods for solving the time-dependent Schrödinger equation, 2012. Bachelor's thesis, Lund University. 32

[96] J. Crank and P. Nicolson. Math. Proc. Camb. Phil. Soc., 43:50, (1947). 32

[97] J. D. Anderson. Computational Fluid Dynamics: The Basics with Applications. McGraw Hill, 1994. 32

[98] M. D. Feit, J. A. Fleck, and A. Steiger. J. Comp. Phys., 47:412, (1982). 32, 33

[99] C. E. Dateo and H. Metiu. J. Chem. Phys., 95:7392, (1991). 32

[100] T. Sorevik, T. Birkeland, and G. Oka. Adv. in Computational Mathematics, 225:56, (2009). 32

[101] B. I. Schneider and N. Nygaard. J. Phys. Chem. A, 106:10773, (2002). 32

[102] R. Kosloff and Tal-Ezer H. Physica A, 284:131, (2000). 32, 37

[103] W. H. Press, S. A. Teukolsky, W. T. Vetterling, and P. B. Flannery. Numerical Recipes in C: The Art of Scientific Computing. Cambridge University Press, 2002. $32,35,56,79,108$ 
[104] P. García González, J. E. Alvarellos, and J. J. García Sanz. Introducción al formalismo de la Mecánica Cuántica. Universidad Nacional de Educación a Distancia, 2005. 33

[105] D. J. Tannor. Introduction to the Quantum Mechanic: A time-dependent perspective. University Science Books, 2007. 33

[106] B. H. Bransden and C. J. Joachain. Introduction to quantum mechanics. Londonman Group UK, 1995. 37

[107] P. de Forcrand and M. Troyer. Computational Quantum Physics. ETH Zürich, 2008. 37,39

[108] E. V. van der Zwan and M. Lein. Phys. Rev. Lett., 108:043004, (2012). 39, 40, 42

[109] M. Frigo and S. G. Johnson. FFTW. http://www.fftw.org/. 42, 65, 71, 73, 75,76

[110] A. Chacón. PULSESMAKER. http://code.google.com/p/pulsemaker/. 43, $57,60,65$

[111] J. Vázquez de Aldana. Interaction with atoms with intense laser field: theoretical study and numerical model, 2001. PhD. thesis, Universidad de Salamanca. 44, 49

[112] L. H. Thomas. Elliptic Problems in Linear Differential Equations over a Network. Columbia University, 1949. 50

[113] A. Chacón, A. De la Calle, and C. Ruiz. BALAS. http://code.google.com/p/ balas/. 51, 60, 78, 79

[114] M. Guizar-Sicairos and J. C. Gutiérrez-Vega. J. Opt. Soc. Am. A, 21:53, (2004). $52,55,79$

[115] M. Puschel, J. Moura, E. Anderson, Z. Bai, L. S. Blackford, et al. Intel @math kernel library. https://software.intel.com/en-us/intel-mkl. 55, 79, 80, 81

[116] J. A. Pérez-Hernández, L. Plaja, and L. Roso. Opt. Express, 17:9891, (2009). 56, 95

[117] C. Ruiz and A. Chacón. QFISHBOWL. http://code.google.com/p/ qfishbowl/. 60 


\section{BIBLIOGRAPHY}

[118] C. Ruiz, A. de la Calle, and A. Chacón. ASSAM. http://code.google.com/p/ qfishbowl/. 60

[119] A. Chacón and R. Morán. Qsphere. http://code.google.com/p/ quantum-sphere-tdse/. 60,80

[120] A. Chacón, R. Morán, and C. Ruiz. Quantum Simulation Lab. http://www . quantumsimlab.es/. $67,69,71,72,80,82$

[121] Advanced Simulation and Computing. VisIt Software: Visualization Tool. https: //wci.llnl.gov/simulation/computer-codes/visit/. 78,81

[122] M. R. Hermann and J. A. Fleck. Phys. Rev. A., 38:6000, (1988). 80, 81

[123] Ch. E. Dateo and H. Metiu. J. Chem. Phys., 95:7392, (1991). 80

[124] A. G. Borisov. J. Chem. Phys., 114:7770, (2001). 80

[125] P. J. Kostelec and D. N. Rockmore. SpharmonicKit. http://www. cs . dartmouth. edu/\%7Egeelong/sphere/. 80,81

[126] P. Emma, R. Akre, J. Arthur, et al. Nature Photonics, 4:641, (2010). 83

[127] Z. Huang and K.-J. Kim. Phys. Rev. STAB, 10:034801, (2007). 83

[128] E. M. Kosik, L. Corner, A. S. Wyatt, et al. J. Mod. Opt., 52:361, (2005). 83

[129] M. Takeda, H. Ina, and S. Kobayashi. J. Opt. Soc. Am., 72:156, (1982). 84, 86, 123

[130] M. P. Rimmer and J. C. Wyant. Appl. Opt., 14:142, (1975). 84, 121, 123

[131] J. Hartmann. Objektivuntersuchungen. Z. Instrumentenkd, 1904. 84, 121

[132] E. Gaviola. J. Opt. Soc. Am., 26:163, (1936). 84, 121

[133] A. M. Nugumanov, R. V. Smirnov, and V. I. Sokolov. Quantum Electronics, 435:5, (2000). 84

[134] J. Primot and L. Sogno. J. Opt. Soc. Am., 12:2679, (1995). 84

[135] F. X. Kärtner, C. E. Ascheron, H. J. Koelsch, and W. Skolaut. Few-cycle laser pulse generation and its applications in Characterization of ultrashort optical pulses. Springer, 2004. 86 
[136] M. E. Anderson, A. Monmayrant, S.-P. Gorza, et al. Laser Phys. Lett., 5:259, (2008). 86

[137] O. R. Wood II, W. T. Silfvast, W. K. Tom, et al. Appl. Phys. Lett., 53:654, (1988). 86

[138] U. Frühling, M. Wieland, M. Gensch, et al. Nature Photonics, 3:523, (2009). 86

[139] V. S. Yakovlev, J. Gagnon, N. Karpowicz, et al. Phys. Rev. Lett., 105:073001, (2009). 88, 89, 109

[140] M. F. Ciappina and T. Kirchner. Comp. Phys. Comm., 183:1832, (2012). 90, 108,125

[141] W. Gropp and E. Lusk. The message passing interface (MPI) standard. Springer, 2004. www.mcs.anl.gov/research/projects/mpi/. 95

[142] J. Sanders and E. Kandrot. CUDA by example, an introduction to general-purpose GPU programming. Addison Wesley, 2010. 95

[143] T. Witting, D. R. Austin, and I. A. Walmsley. Opt. Letters, 34:881, (2009). 97

[144] R. Thomas. Attosecond electron wave packet interferences, 2008. Doctoral Thesis Sweden. 99

[145] O. Smirnova, A. S. Mouritzen, S. Patchkovskii, and M. Y. Ivanov. J. Phys. B, 40:F197, (2007). 102

[146] M. Ivanov and O. Smirnova. Phys. Rev. Lett., 107:213605, (2011). 102, 103

[147] J. Mauritsson, P. Johnsson, E. Gustafsson, et al. Phys. Rev. Lett., 97:013001, (2006). 105

[148] U. Graf, M. Fieß, M. Schultze, et al. Opt. Express, 16:18956, (2008). 105

[149] J. W. Cooper. Phys. Rev., 128:681, (1962). 109

[150] D. R. Austin, T. Witting, and I. A. Walmsley. J. Opt. Soc. Am. B, 26:1818, (2009). 117, 142

[151] M. E. Anderson, L. E. E. de Araujo, and I. A. Kosik, E. M. Walmsley. Appl. Phys. B, 70:S85-S93, (2000). 117

[152] J. Primot. Opt. Comm., 222:81, (2003). 121, 123 


\section{BIBLIOGRAPHY}

[153] J. D'Errico. Inverse (integrated) gradient, (2006). http://www.mathworks.com/ matlabcentral/fileexchange. 135, 137

[154] J. R. Birge, R. Ell, and F. X. Kärtner. Opt. Lett., 31:2063, (2006). 142

[155] C. A. A. de Carvalho and H. M. Nussenzveig. Phys. Rep., 364:83, (2002). 152, 204, 205

[156] J. Su, N. Hongcheng, A. Becker, and A. Jaron-Becker. Phys. Rev. A, 88:023413, (2013). 152, 208

[157] S. Nagele, R. Pazourek, M. Wais, et al. Time-resolve photoemission using attosecond streaking, (2014). http://arxiv.org/abs/1401.6328. 153, 202, 203, 205

[158] R. Pazourek, S. Nagele, J. Feist, and J. Burgdöfer. Phys. Rev. Lett., 108:163001, (2012). 154

[159] R. Pazourek, S. Nagele, and J. Burgdöfer. Faraday Discuss., 163:353, (2013). $159,161,171$

[160] Y. J. Chen, L. B. Fu, and J. Liu. Phys. Rev. Lett., 111:073902, (2013). 161

[161] NIST. National Institute of Standards and Technology, (1901). http://cccbdb. nist.gov/. 162, 177

[162] E. Lötsedt, T. Kato, and K. Yamanouchi. J. Chem. Phys., 138:104304, (2013). 176,191

[163] A. Etches and L. B. Madsen. J. Phys. B: At. Mol. Opt. Phys., 43:155602, (2010). 177,179

[164] R. Pazourek, S. Nagele, K. Doblhoff-Dier, et al. J. Phys. B: At. Mol. Opt. Phys., 388:012029, (2012). 189

[165] J. Su, N. Hongcheng, A. Becker, and A. Jaron-Becker. J. Mod. Opt., 60:1484, (2013). 203, 207, 208

[166] S. Nagele, R. Pazourek, J. Feist, and J. Burgdörfer. Phys. Rev. A, 85:033401, (2012). 204 